\title{
IN VITRO ASSAYS FOR HAZARD IDENTIFICATION OF NANOPARTICLES
}

Samantha K. Kloet 


\section{Thesis committee}

\section{Promotor}

Prof. Dr I.M.C.M. Rietjens

Professor of Toxicology

Wageningen University

\section{Co-promotors}

Dr J. Louisse

Assistant professor, Sub-department of Toxicology

Wageningen University

Dr N.W. van den Brink

Associate professor, Sub-department of Toxicology

Wageningen University

\section{Other members}

Prof. Dr A.A. Koelmans, Wageningen University

Prof. Dr A.H. Piersma, Utrecht University

Dr A.G. Oomen, National Institute for Public Health and the Environment (RIVM), Bilthoven

Dr T. Hamers, VU University Amsterdam

This research was conducted under the auspices of the Graduate School VLAG (Advanced studies in Food Technology, Agrobiotechnology, Nutrition and Health Sciences). 


\title{
IN VITRO ASSAYS FOR HAZARD IDENTIFICATION OF NANOPARTICLES
}

\author{
Samantha K. Kloet
}

\section{THESIS}

submitted in fulfilment of the requirements for the degree of doctor

at Wageningen University

by the authority of the Rector Magnificus

Prof. Dr A.P.J. Mol,

in the presence of the

Thesis Committee appointed by the Academic Board

to be defended in public

on Wednesday $7^{\text {th }}$ of December 2016

at 11 a.m. in the Aula. 
Samantha Kristina Kloet

In vitro assays for hazard identification of nanoparticles 211 pages.

$\mathrm{PhD}$ thesis, Wageningen University, Wageningen, NL (2016)

With references, with summary in English

ISBN: 978-94-6257-941-5

DOI: $10.18174 / 390912$ 


\section{TABLE OF CONTENTS}

Chapter 1 General introduction, aim and outline of the thesis

Chapter 2 Progress and future of in vitro models to study translocation of nanoparticles

Chapter 3 Translocation of positively and negatively charged polystyrene 79 nanoparticles in an in vitro placental model

Chapter 4 Assessment of the potential developmental toxicity of positively and 105 negatively charged polystyrene nanoparticles using the embryonic stem cell differentiation assay

Chapter 5 Effects of nanoparticle forms of metal (oxide) food additives and related nanoparticles on cell viability, TNF- $\alpha$ production and mitochondria-related parameters in RAW264.7 macrophages

Chapter 6 Cellular interactions of silver nanoparticles with systematic variation in size and surface coating with macrophage RAW 264.7 cells

Chapter 7 General discussion, future perspectives and conclusions

Chapter 8 Summary

About the author

List of publications 


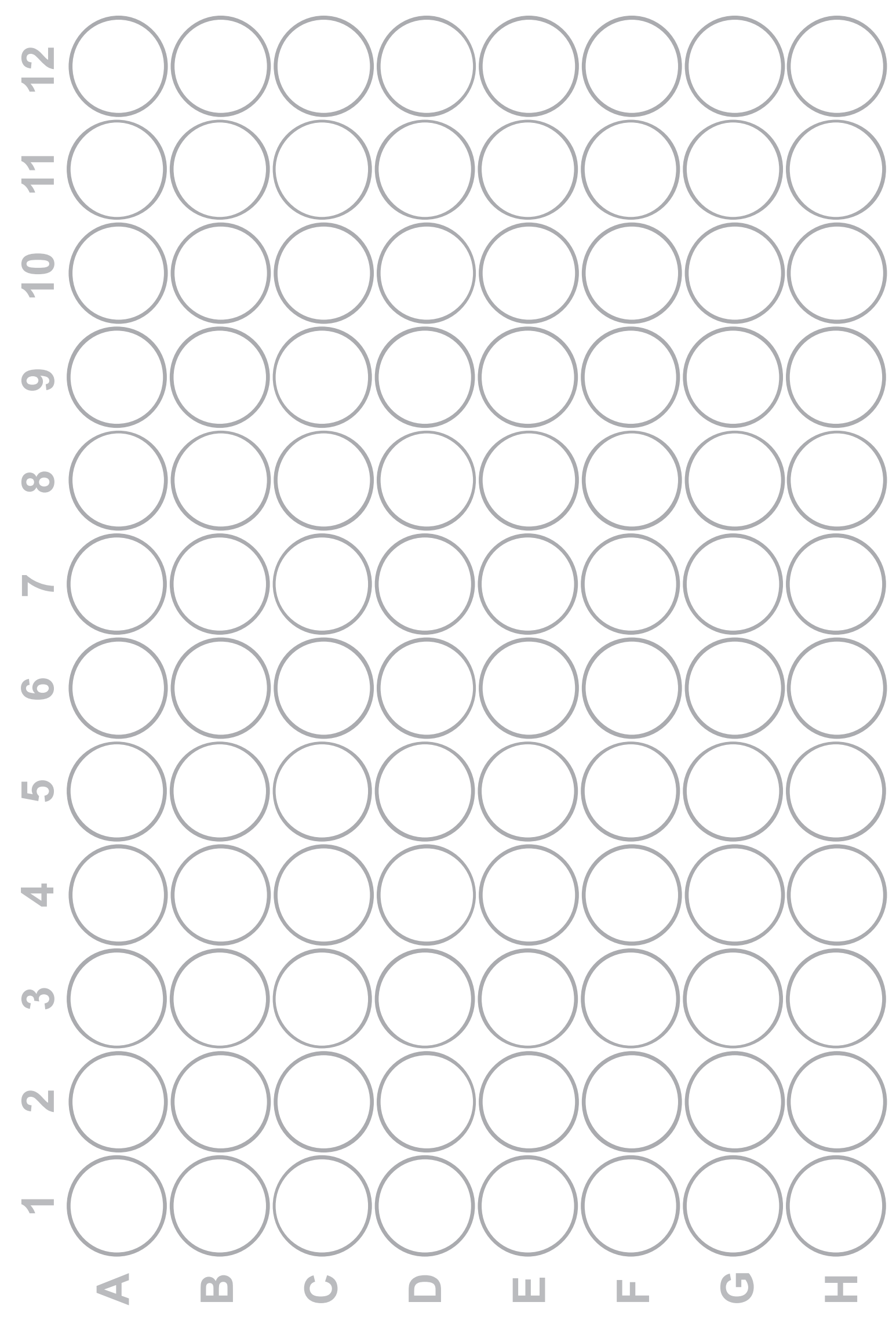




\section{Chapter 1}

General introduction, aim and outline of the thesis 


\section{BACKGROUND}

In recent years technological innovations have increasingly developed and applied nanomaterials (NMs) which are structures that have at least one external dimension in the nanoscale (1-100 nm) (Stone et al. 2009). In this thesis the focus lies on nanoparticles (NPs) which are NMs with all three external dimensions (length, height, width) in the nanoscale (ISO/TS 80004-2:2015). Due to their small size and relatively large surface area to volume ratio, NPs have physicochemical characteristics different from those of the bulk material, such as different thermal, mechanical, electrical, chemical, catalytic and optical properties, which makes engineered NPs so interesting for applications (Auffan et al. 2009, Kango S. 2013). For example, bulk gold is considered to be inert but gold NPs are very effective catalysts (Auffan et al. 2009). Due to their specific properties, engineered NPs offer numerous interesting opportunities in many sectors of society including medicine, agriculture and food technology.

Since NPs are incorporated more and more in a variety of consumer products, it is likely that the general public may be exposed to NP-containing products such as personal care products, food and food packaging materials, textiles and medicine. In order to assess whether exposure to these NPs may pose a human health risk, NPs should be assessed for their safety. Insight into the potential human health risks posed by NPs is essential for sustainable development and safe use of innovative products based on these materials. To develop such insights, toxic properties (hazards) of NPs should be identified and dose-response relationships established. Despite the fact that there are a number of publications concerning undesirable effects of NPs, various gaps still exist in the knowledge on the intrinsic hazards of NPs, the potential human exposures to NPs and the relationship between exposure and adverse health effects (risks) of NPs (Oberdorster et al. 2007, Dhawan et al. 2010, Hussain et al. 2015). Existing information on potential adverse health effects is mainly based on animal experiments, although in vitro studies with cell lines have recently added to the insight in potential hazards of NPs. NPs have been shown, for example, to induce cytotoxic, genotoxic, inflammatory and oxidative stress responses in different mammalian cell lines (Nel et al. 2006, Elsabahy et al. 2013, Watson et al. 2014).

There is no doubt that the safety testing of all these materials requires a high number of experimental animals given the large numbers and variety of NPs developed and applied. Testing all these NPs in animal bioassays is undesirable for scientific, ethical and societal reasons. There is a strong societal demand to reduce the use of experimental animals for safety testing of chemicals, including NPs. In order to reduce in vivo testing, alternative methods for testing of chemicals are currently being developed and validated (Van der Jagt et al. 2004, Macharia Theuri et al. 2016). It is desirable that the safety and risk assessment of NPs would be largely based on studies using in vitro models instead of in vivo models as this would also reduce costs and time needed to test the large numbers of NPs. 
Use of in vitro models may also help to define structure activity relationships and facilitate read-across within classes of NPs. Previous research has indicated that NP toxicity can be related to a certain extent to their physicochemical properties (Fourches et al. 2010). Different specific biological responses resulting from exposure to NPs, such as reduction in cell viability, are shown to be affected by NP physicochemical properties, such as size, surface charge and surface area (Duran et al. 2015, Recordati et al. 2016). It would be of great value to get more insight in these relationships, not only to facilitate read across within classes of NPs, but also to be able to set priorities for safety testing and/or to develop safer NPs.

\section{AIM OF THE THESIS}

The aim of the present thesis was to investigate the potential of in vitro alternative testing strategies to detect hazards of NPs, focusing on toxicokinetic as well as toxicodynamic endpoints. Toxicokinetic studies focused on translocation of NPs across in vitro models of the placental barrier, while toxicodynamic studies were directed at two endpoints that represent potential hazards of NPs that have not been well characterized yet: developmental toxicity and immunotoxicity. The model NPs and in vitro model systems used in the present thesis are described in more detail in the following sections.

\section{NANOPARTICLES}

The next sections provide background information about the different types of NPs that have been used in this thesis. A first group of model NPs consisted of polystyrene NPs (PS-NPs). A second group of model NPs consisted of metal and metal oxide NPs.

\section{Polystyrene NPs (PS-NPs)}

During the last decades, NPs made of polymers like polystyrene are increasingly used in food packaging due to their functionality, light weight, ease of processing and low costs (Arora et al. 2010). PS-NPs are also used in industrial applications, including sensors and drug delivery systems (Phosphorex, Simon et al. 2008, Arora and Padua 2010, Lee et al. 2014, Chiu et al. 2015). At present it is unknown whether humans are exposed to such PS-NPs due to e.g. migration into food and drinks (Chaudhry et al. 2008). It is also not clear whether such a migration would have a consequence for the safety of the food.

Fluorescent PS-NPs are often used as model NPs in nanotoxicological studies to investigate interactions between NPs and cells because of their commercial availability, high quality, easy detection by means of fluorescence measurements and wide variety of available sizes and surface chemistries (surface charges) (Varela et al. 2012). PS-NPs have been reported to enter different cell types like renal cells (Monti et al. 2015), macrophages (Xia et al. 2008), hepatocytes 
(Johnston et al. 2010), gastric cells (Forte et al. 2016) and lung cells (Geys et al. 2006, Yacobi et al. 2008, Salvati et al. 2011, Deville et al. 2015). Results from these studies have shown that the in vitro internalisation of NPs depends on several factors like cell type, NP size, surface charge as well as the presence or absence of serum in the cell culture medium. Evaluation of studies with PS-NPs with different size (50 and $100 \mathrm{~nm}$ ) and charge (negative and positive) in an in vitro intestinal cell model, suggests that ingested PS-NPs, depending on their size and charge, could potentially translocate across the intestinal barrier (Walczak et al. 2014).

Although polystyrene itself is considered to be inert, several studies have found toxic effects of PS-NPs. After internalisation in human gastric adenocarcinoma epithelial cells, PS-NPs of 44 and $100 \mathrm{~nm}$ affected gene expression, and caused inflammatory responses and morphological alterations, especially for the smaller sized PS-NPs (Forte et al. 2016). In another study, $20 \mathrm{~nm}$ carboxylated PS-NPs were taken up passively, stimulated IL- 8 secretion, and induced oxidative burst in human monocytes while $1000 \mathrm{~nm}$ PS-NPs entered cells both passively and actively (Prietl et al. 2014). The effect of size has also been demonstrated by Bhattacharjee et al. who investigated polymer NPs of 45 and $90 \mathrm{~nm}$. The $45 \mathrm{~nm}$ NPs showed a higher response in terms of cytotoxicity, ROS production and TNF- $\alpha$ release, compared to the $90 \mathrm{~nm}$ NPs (Bhattacharjee et al. 2012). Surface charge is another factor that could determine cytotoxicity of NPs (Bhattacharjee et al. 2010, Frohlich 2012). In the present thesis, aminated PS-NPs (positively charged) and carboxylated PS-NPs (negatively charged) were used. The present thesis studied whether these differences in surface charge would affect their toxicokinetic and toxicodynamic characteristics.

\section{Metal and metal oxide NPs}

The other model NPs used in the present thesis were metal and metal oxide NPs. Several metal and metal oxide NPs are possible constituents of approved food additives. Food additives, such as titanium dioxide $\left(\mathrm{TiO}_{2}\right)(\mathrm{E} 171)$, iron(III) oxide $\left(\mathrm{Fe}_{2} \mathrm{O}_{3}\right)(\mathrm{E} 172)$, and silicon dioxide $\left(\mathrm{SiO}_{2}\right)(\mathrm{E} 551)$ consist of metal oxide particles of variable sizes. Also metal NPs may be present in food additives, and for this silver (Ag) (E174) is an example. At present the specifications of these food additives do not exclude the presence of the compounds in their nanoform, and food additives are excluded from the regulation that states that all ingredients that are present in nano-form should be indicated in the list of ingredients as 'nano' (2011). In addition to the food-borne NPs, also some related metal oxide NPs were used in the present thesis. These included manganese oxide $\left(\mathrm{Mn}_{2} \mathrm{O}_{3}\right)$, copper oxide $(\mathrm{CuO})$, chromium oxide $\left(\mathrm{Cr}_{2} \mathrm{O}_{3}\right)$, cobalt oxide $\left(\mathrm{Co}_{3} \mathrm{O}_{4}\right)$ and nickel oxide $(\mathrm{NiO}) \mathrm{NPs}$, to which humans may be exposed via products like paints, coatings, batteries, catalysts and construction materials (Siddiqui et al. 2012, Tavares et al. 2014, Dang et al. 2015). 


\section{Titanium dioxide $\left(\mathrm{TiO}_{2}\right)$.}

$\mathrm{TiO}_{2}$ is used in food as a colorant (E171), and as such may end up in food in the nanoform (Periasamy et al. 2015, Grande et al. 2016). Chewing gum and candies contain the highest amount of particulate $\mathrm{TiO}_{2}$. Of the chewing gum and candies many tested samples contained 0.01 to $1 \mathrm{mg}$ titanium per serving (Weir et al. 2012, Peters et al. 2014). $\mathrm{TiO}_{2} \mathrm{NPs}$ are also used in glazes, paper, fibers, plastics, pharmaceuticals, cosmetics, sunscreens and toothpastes due to their ability to confer opacity and whiteness (Weir et al. 2012). It has been shown that 25-38\% of the $\mathrm{TiO}_{2}$ of a food-grade $\mathrm{TiO}_{2}$ was present as NPs smaller than $200 \mathrm{~nm}$ (Weir et al. 2012). $\mathrm{TiO}_{2} \mathrm{NPs}$ are normally present as a mixture of anatase and rutile crystal forms, of which the anatase form has been found to be more chemically reactive (Warheit et al. 2007).

Concerns have been raised that $\mathrm{TiO}_{2} \mathrm{NPs}$ may present a different bioactivity than bulk $\mathrm{TiO}_{2}$ and cause adverse effects to human health (Shi et al. 2013). It has been found that $\mathrm{TiO}_{2} \mathrm{NPs}$ can induce genotoxicity, cytotoxicity, formation of reactive oxygen species (ROS) and induce cytokine production as demonstrated in vitro and in vivo (Gerloff et al. 2012, Montiel-Davalos et al. 2012, Shi et al. 2013). Also exposure to a food grade $\mathrm{TiO}_{2}$ which included about $25 \%$ of the $\mathrm{TiO}_{2}$ as NPs $<100 \mathrm{~nm}$, has been found to cause a loss of microvilli of Caco- $2_{\text {BBel }}$ cells (Faust et al. 2014). Although $\mathrm{TiO}_{2}$ NPs have been studied extensively in recent years, a lot of issues concerning possible health effects still remain to be elucidated to support risk assessment and management (Shi et al. 2013).

\section{Iron oxide $\left(\mathrm{Fe}_{2} \mathrm{O}_{3}\right)$.}

$\mathrm{Fe}_{2} \mathrm{O}_{3}$ is used in food products as coloring agent (E172), in paints and in the cosmetic and personal care industry, to add color to products like eye shadow, lipstick and mineral make-up. In recent years there has also been an increased interest for the use of $\mathrm{Fe}_{2} \mathrm{O}_{3} \mathrm{NPs}$ for biomedical, magnetic, catalytic and electronic applications (Valdiglesias et al. 2016). New applications are for example drug delivery systems or magnetic resonance imaging contrast agents (Baetke et al. 2015). Together with these promising applications, concerns have been raised regarding the onset of unexpected adverse health effects following exposure. In vitro and in vivo studies report on several adverse effects of $\mathrm{Fe}_{2} \mathrm{O}_{3}$ NPs, such as a decrease in cell viability (Karlsson et al. 2009, Ying et al. 2010), increased ROS production (Alili et al. 2015), induction of apoptosis (Kim et al. 2013), cell cycle alterations (Wu et al. 2011), cell membrane disruptions (Rajiv et al. 2016), cytoskeleton modifications (Wu et al. 2008) and autophagy (Shi et al. 2015). However, a recent review concluded that the potential human health effects of $\mathrm{Fe}_{2} \mathrm{O}_{3}$ NPs remain unknown, since the currently available information on their potential toxicity is still scarce and contradictory, and human epidemiological studies do not exist (Valdiglesias et al. 2016).

\section{Silicon dioxide $\left(\mathrm{SiO}_{2}\right)$.}

$\mathrm{SiO}_{2}$ is used as an anti-caking additive in several food products (Chaudhry et al. 2008, Dek- 
kers et al. 2013). Coffee creamers, instant soups and seasoning mixes are products that contain the highest amount of nano-sized $\mathrm{SiO}_{2}$ (Dekkers et al. 2011). Analysis of isolated $\mathrm{SiO}_{2}$ samples from commercial food products showed the presence of $\mathrm{SiO}_{2} \mathrm{NPs}$ of about 10-50 nm in size (Athinarayanan et al. 2014). Also, it was demonstrated that $33 \%$ of the food additive $\mathrm{SiO}_{2}$ (E551) consisted of 10- to 200-nm spherical particles (Dekkers et al. 2011). The health risks of using nano-sized $\mathrm{SiO}_{2}$ particles as food additives are largely unknown.

When food products containing $\mathrm{SiO}_{2}$ were tested in an in vitro digestion model, which simulated the conditions of the human gastrointestinal tract, it was found that $\mathrm{SiO}_{2}$ NPs $(5-200$ $\mathrm{nm}$ ) were still present in the intestinal content upon 4 hours and 15 minutes of incubation (Peters et al. 2012). This indicates that it is most likely that upon consumption of foods containing $\mathrm{SiO}_{2}$, the gut epithelium is exposed to $\mathrm{SiO}_{2} \mathrm{NPs}\left(5-200 \mathrm{~nm}\right.$ ) (Peters et al. 2012). $\mathrm{SiO}_{2}$ NPs can translocate across the intestinal barrier, as demonstrated in a rat in vivo study in which 6.6-9.7\% of $\mathrm{SiO}_{2}$ NPs of 20 and $100 \mathrm{~nm}$ were absorbed into the systemic circulation when orally administered at a single dose of 500 or $1000 \mathrm{mg} / \mathrm{kg}$ bw (Lee et al. 2014).

Several in vitro and in vivo studies have shown adverse effects of $\mathrm{SiO}_{2} \mathrm{NPs}$. Yamashita et al. demonstrated that $70 \mathrm{~nm} \mathrm{SiO}{ }_{2}$ NPs reduced the growth of fetuses in pregnant mice upon intravenous injection, while the 300 and $1000 \mathrm{~nm}$ particles did not induce these effects (Ya-

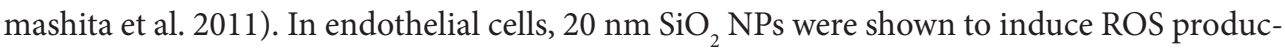
tion which resulted in apoptosis via JNK/p53 dependent mitochondrial pathways (Liu et al. 2010). Exposure of bone marrow-derived dendritic cells to $\mathrm{SiO}_{2} \mathrm{NPs}$ (14 nm) induced partial maturation of the cells, activation of the inflammasome and apoptosis in a substantial fraction of cells (Winter et al. 2011). Human lung normal fibroblast treated with high concentrations of isolated $\mathrm{SiO}_{2}$ containing NPs (10-50 nm), showed cellular damage, mitochondrial membrane potential depletion, and ROS generation. The cell cycle distribution was altered and the expression of antioxidant enzymes and stress response proteins were modulated (Athinarayanan et al. 2014).

\section{Silver (Ag).}

The food additive silver (E174) is used as coloring agent to give a metallic appearance to for example cake decorating sweets. Silver NPs are used in food supplements, in the form of aqueous suspensions of Ag NPs that claim to enhance the body's immune system and to have antimicrobial activity (Griffith et al. 2015). Furthermore, Ag NPs are used as antibacterial coatings in refrigerators and food packaging materials, wound dressings, clothes and deodorants (Nanoparticle Products Inventory). In vitro studies report on various adverse effects of Ag NPs, including for example a decrease in cell viability (McShan et al. 2014), increased ROS production (Rahman et al. 2009), DNA damage (Huk et al. 2014), cell cycle alterations (Asharani et al. 2009), apoptosis (Foldbjerg et al. 2009) and inhibition of stem cell differenti- 
ation (Park et al. 2011). In vivo studies with Ag NPs have been done with rats (Vandebriel et al. 2014, Boudreau et al. 2016, Garcia et al. 2016) and mice (Su et al. 2013). In a 28-day repeated-dose toxicity study with rats, intravenously administration of Ag NPs induced suppression of the functional immune system (Vandebriel et al. 2014). Factors known to influence toxicity are concentration, dispersion, size and surface functionalization of the Ag NPs (Duran et al. 2015, Recordati et al. 2016).

\section{Manganese oxide $\left(\mathrm{Mn}_{2} \mathrm{O}_{3}\right)$.}

Human exposure to $\mathrm{Mn}_{2} \mathrm{O}_{3}$ NPs is not so much via the food, but is more likely to take place as occupational exposure since $\mathrm{Mn}_{2} \mathrm{O}_{3}$ NPs are mainly used in industry, for example as composite in wastewater treatment, as catalysts, sensors, electronic components or voltage sensitive material (Negahdary et al. 2015). So far, a limited number of studies investigating the effects of $\mathrm{Mn}_{2} \mathrm{O}_{3}$ has been performed. Some in vitro studies classified $\mathrm{Mn}_{2} \mathrm{O}_{3}$ NPs as one of the most toxic metal oxide NPs and it has been hypothesized that oxidative stress can be a mechanism underlying their cytotoxicity (Zhang et al. 2012, Liu et al. 2013). Recently, $70 \mathrm{~nm} \mathrm{Mn} \mathrm{O}_{3} \mathrm{NPs}$ at a dose of $400 \mathrm{ppm}$ in the diet were found to reduce sex hormone levels and sperm production and caused testicular damage in Wistar adult male rats (Negahdary et al. 2015).

\section{Copper oxide (CuO).}

$\mathrm{CuO}$ NPs are used in catalysts, gas sensors, conductors, batteries and for applications in antimicrobial textiles, paints and plastics metallic coatings (Ren et al., 2009; Dastjerdi and Montazer, 2010; Delgado et al., 2011; Chang, Zhang et al. 2012). Exposure to CuO is mainly through inhalation (Ahamed et al. 2015). In vitro studies have shown that $\mathrm{CuO}$ NPs can cause cytotoxicity, DNA damage, ROS production, and inflammatory and genotoxic effects (Karlsson et al. 2008, Karlsson et al. 2009, Perreault et al. 2012, Zhang et al. 2012). CuO NPs are reported to cause oxidative stress in cells (Karlsson et al. 2008, Zhang et al. 2012, Mitra et al. 2013).

Chromium oxide $\left(\mathrm{Cr}_{2} \mathrm{O}_{3}\right)$.

$\mathrm{Cr}_{2} \mathrm{O}_{3}$ NPs are used as a pigment in paints, inks, and glasses. It is the colourant in "chrome green" and "institutional green" (Senapati et al. 2015). Furthermore $\mathrm{Cr}_{2} \mathrm{O}_{3}$ NPs are used in a wide variety of applications such as: coating materials for thermal protection, wear resistance materials, solar energy application, digital recording system and electrochromic material (Abdullah et al. 2014, Meenambika et al. 2014). Exposure to $\mathrm{Cr}_{2} \mathrm{O}_{3}$ NPs is mainly through inhalation (Senapati et al. 2015). In vitro studies have shown that in human lung epithelial cells, exposure to $30 \mathrm{~nm} \mathrm{Cr}_{2} \mathrm{O}_{3}$ NPs caused DNA damage, which was investigated by the comet assay and the cytokinesis block micronucleus assay (Senapati et al. 2015). In L929 cells, $37 \mathrm{~nm}$ $\mathrm{Cr}_{2} \mathrm{O}_{3}$ NPs had significant cytotoxic effects, induced ROS production and caspase- 3 induction, indicating that exposure to $\mathrm{Cr}_{2} \mathrm{O}_{3}$ NPs induced apoptosis (Alarifi et al. 2016). 
Cobalt oxide $\left(\mathrm{Co}_{3} \mathrm{O}_{4}\right)$.

$\mathrm{Co}_{3} \mathrm{O}_{4}$ NPs find applications in catalysts, sensors, magnetism, energy storage and in pigments (Papis et al. 2009). Since $\mathrm{Co}_{3} \mathrm{O}_{4}$ NPs are used in automotive exhaust catalysts, the NPs may get released into the air, and thus may be inhaled by humans (Verstraelen et al. 2014). In vitro studies have shown that exposure of HepG2 cells to $\mathrm{Co}_{3} \mathrm{O}_{4}$ NPs resulted in an induction of cytotoxicity and genotoxicity through ROS formation and oxidative stress (Alarifi et al. 2016). In another study using human lymphocytes, a decrease in cell viability and an increase in cell membrane damage was observed after exposure to $\mathrm{Co}_{3} \mathrm{O}_{4}$ NPs. In addition chromosomal aberrations were observed in human lymphocytes exposed to $100 \mu \mathrm{g} / \mathrm{ml} \mathrm{Co}_{3} \mathrm{O}_{4} \mathrm{NPs}$ for 24 hours (Rajiv et al. 2016). One other study investigated both the in vitro effects of $60 \mathrm{~nm} \mathrm{Co} \mathrm{O}_{4} \mathrm{NPs}$ in normal PBMCs and the in vivo effects in Swiss mice that were subcutaneously injected by a three-days interval for 15 and 30 days with $\mathrm{Co}_{3} \mathrm{O}_{4}$ NPs (Chattopadhyay et al. 2015). $\mathrm{Co}_{3} \mathrm{O}_{4}$ NPs induced significantly the intracellular production of ROS and cytokines and apoptosis in normal PBMCs exposed in vitro. The in vivo results showed toxicity demonstrated by a significant rise in lactate dehydrogenase (LDH), serum glutamate oxalate transaminase (SGOT) and creatinine level in the $200-1000 \mu \mathrm{g} / \mathrm{kg}$ bw treated groups (Chattopadhyay et al. 2015).

\section{Nickel oxide (NiO).}

While occupational nickel exposure is a known cause of pulmonary alveolitis, fibrosis, and cancer, the health risks of NiO NPs are not so well understood (Glista-Baker et al. 2014). $\mathrm{NiO}$ is an important industrial material used for solar cells, lithium-ion batteries, electronic components, as pigment for glasses, catalysts and ceramic materials (Song et al. 2008, Horie et al. 2011). Compared to bulk NiO, NiO NPs possess unique properties such as early oxidation and melting phenomena, which can be used in industrial products promoting innovative applications (Song et al. 2008, Oukarroum et al. 2015). Possible exposure to NiO NPs can occur via inhalation (Shimada et al. 2009). In vitro studies have reported effects of NiO NPs exposure including cytotoxicity (Ada et al. 2010), oxidative stress (Horie et al. 2011), DNA damage (Kawanishi et al. 2002), apoptosis (Siddiqui et al. 2012) and induction of inflammatory cytokines (Morimoto et al. 2010). Some in vivo studies have demonstrated that NiO NPs of $26 \mathrm{~nm}$ (Nishi et al. 2009, Morimoto et al. 2010), $27 \mathrm{~nm}$ (Ogami et al. 2009), and $20 \mathrm{~nm}$ (Horie et al. 2011) were able to induce inflammatory effects after intratracheally instillation in rats.

\section{IN VITRO MODELS FOR THE STUDY OF TOXICOKINETICS OF NANOPARTI- CLES}

In the present thesis, first the use of in vitro models for studies on the toxicokinetics of NPs is investigated. Toxicokinetics describe the uptake and fate of chemicals and NPs in the body as a result of absorption, distribution, metabolism, and excretion (ADME) processes, which together determine the internal exposure and the potential adverse health effect. The four main 
routes via which NPs may enter the human body are oral, inhalation, dermal and systemic intravenous (IV) injection (Oberdorster et al. 2007, Krug et al. 2011). Several studies have indicated that translocation of NPs across the gut, lung and skin barriers is possible, enabling NP uptake upon oral, inhalation and dermal exposure, respectively (Landsiedel et al. 2012). Also, NPs have been shown to translocate across other barriers, such as the blood-brain barrier and the placental barrier (Landsiedel et al. 2012). In order to estimate the in vivo internal exposure, several in vitro models have been used to study NP translocation across the lung, gut, skin, blood-brain and placental barrier (Mahler et al. 2012, Prow et al. 2012, George et al. 2015, Poulsen et al. 2015). A detailed evaluation of the performance of such in vitro models is presented in chapter 2 of this thesis.

Since part of the toxicodynamic studies of the present thesis were directed at testing the possible developmental toxicity of NPs, an important toxicokinetic aspect studied in the present thesis was placental translocation of NPs. At present, research on placental translocation of nanomaterials and their effects on the placenta and the developing fetus is still in its infancy (Saunders 2009, Buerki-Thurnherr et al. 2012, Juch et al. 2013). The perfused isolated human placenta ex vivo model which maintains the complexity of the intact placenta, has often been used to study placental transfer (Saunders 2009). A drawback of the isolated ex vivo human placenta model is that experiments with this model are technically challenging and require large quantities of substances for testing. Therefore, models using placental cells in an in vitro transwell system are being developed that may serve as in vitro alternatives for the isolated human placenta ex vivo model. Generally, these models are easier to handle and require less test material. Available human placental choriocarcinoma cell lines for such models include BeWo b30, Jar and JEG-3 cell lines, which have been applied to study placental translocation of a variety of drugs and compounds (Manley et al. 2005, Ikeda et al. 2011, Blazquez et al. 2014). The BeWo b30 cell line is most commonly used in in vitro models of the placental barrier (Saunders 2009) and was also applied in the present thesis. This cell line was derived from a human choriocarcinoma and can be grown on transwell inserts to form a cell layer that separates an apical from a basolateral compartment, representing the maternal and the fetal side, respectively.

A few studies have applied the BeWo b30 transwell model to study translocation of NPs, including PEGylated gold NPs of 10-30 nm (Myllynen et al. 2008), iron oxide and silica NPs of 23-38 nm (Correia Carreira et al. 2013), dexamethasone loaded Poly lactic-co-glycolic acid (PLGA) NPs of 140-289 nm (Ali et al. 2013), rhodamine labelled silica NPs of 25 and 50 $\mathrm{nm}$ (Sonnegaard Poulsen et al. 2013) and neutral PS-NPs of 50 and $100 \mathrm{~nm}$ (Cartwright et al. 2012). The $50 \mathrm{~nm}$ PS-NPs were translocated to a higher extent to the fetal compartment than the $100 \mathrm{~nm}$ PS-NPs, indicating that transport of PS-NPs in this in vitro model of the placental barrier is, like in the ex vivo placental model (Wick et al. 2010), dependent on the 
size of the NPs (Cartwright et al. 2012). These studies focused on the effect of size on the potential translocation, while no studies have been performed on the effects of surface charge, another important property of NPs driving their toxicokinetics. In the present thesis, we aimed therefore to assess the influence of surface charge on placental translocation. For this, the translocation of positively and negatively charged $50 \mathrm{~nm}$ PS-NPs was studied in the BeWo b30 placental model.

\section{IN VITRO MODELS FOR THE STUDY OF TOXICODYNAMICS OF NANOPARTI- CLES}

In the present thesis toxicodynamic studies were directed at two endpoints that represent potential hazards of NPs that have not yet been well characterized. These included developmental toxicity and immunotoxicity.

\section{Developmental toxicity of nanoparticles}

Developmental toxicity refers to the adverse effects of compounds on developmental processes including the induction of structural abnormalities (malformations), growth retardation, death, and behavioural and functional abnormalities. To date, data on developmental toxicity of NPs are limited and remain insufficient as a basis for risk assessment for pregnant women and their children (Powers et al. 2013, Hougaard et al. 2015). For NPs to reach the fetus, they first need to translocate at the portal of entry to the systemic circulation and then traverse the placental barrier. NPs have been shown to be able to pass physiological barriers, such as that of the intestines and the placenta (Wick et al. 2010, Walczak et al. 2015), indicating that systemic exposure, including fetal exposure is feasible and that the fetus is hence a potential target of NPs.

To assess possible adverse effects of NPs on the developing fetus, in vivo developmental toxicity studies can be performed in for example rats (Ema et al. 2016, Garcia et al. 2016). However, because of economic and ethical constrains with in vivo studies, alternatives for in vivo toxicity studies are being developed and validated (Jelinek et al. 1985, Fort et al. 2002, zur Nieden et al. 2004, Spielmann et al. 2006, Park et al. 2009, de Jong et al. 2011). Due to the complexity of the developmental process, it is a challenge to develop non-animal based methods that cover the whole developmental process (de Jong et al., 2011). Although the implementation of alternative methods for in vivo toxicity testing in regulatory frameworks is a relatively slow process, several in vitro tests that may function adequately in hazard assessment for developmental toxicity are available.

Currently, three in vitro developmental toxicity tests have been validated according to ECVAM's (European Centre for the Validation of Alternative Methods) criteria: the whole em- 
bryo culture (WEC), the limb bud micromass (MM) and the embryonic stem cell test (EST) (Genschow et al. 2002, Augustine-Rauch et al. 2010). Of these three tests only the EST does not require pregnant animals to obtain embryonic tissue (de Jong et al. 2011). The EST uses pluripotent mouse embryonic stem cells, derived from the inner cell mass of blastocysts. The test is based on the capacity of murine embryonic stem (ES) cells (cell line D3) to differentiate into contracting myocardial cells under specific cell culture conditions (Campagnolo et al., 2013). The treatment-induced inhibition of the formation of beating cardiomyocytes in embryoid bodies (EBs) outgrowth is used as a toxicological endpoint to assess the embryotoxic potential of a compound (Buesen et al., 2004). Disturbances of ES cell differentiation following a treatment indicate an embryotoxic potential, since embryotoxic compounds can interfere with these differentiation processes (ECVAM 2012). The EST is relatively easy to carry out and the endpoints used in the assay do not require extensive knowledge on morphological development (de Jong, 2012). So far, some studies have used the differentiation assay of the EST to test NPs for in vitro developmental toxicity potencies, such as the studies testing amorphous silica NPs (Park et al. 2009), gold and cobalt ferrite NPs (Di Guglielmo et al. 2010), and oxidized single-wall carbon nanotubes (SWCNTs) (Pietroiusti et al. 2011). So far, the EST has never been applied to evaluate the effects of surface charge of NPs on embryotoxicity. The present thesis aims to study the embryotoxicity of differentially charged PS-NPs in the EST, which can be related to the toxicokinetic studies in the in vitro BeWo b30 placental transwell system using the same PS-NPs.

\section{Immunotoxicity of nanoparticles}

A second toxicodynamic endpoint of interest is the potential immunotoxicity of NPs. Given that immune responses are highly complex processes, in vitro models generally focus on specific sub-characteristics of this endpoint. Intestinal and colonic macrophages represent the largest population of mononuclear phagocytes in the body. Gut macrophages are part of the first-line defence mechanisms, playing a major role in the innate immune response (Ma et al. 2003, Smith et al. 2011, Elsabahy and Wooley 2013). NP-induced macrophage cell death may decrease the innate immune response. NPs have been shown to induce inflammatory responses, like induction of proinflammatory cytokines and oxidative stress responses, in different mammalian cell lines which may point at possible immunotoxicity (Nel et al. 2006, Elsabahy and Wooley 2013, Watson et al. 2014). NPs can interact with various components of the immune system and either enhance or inhibit its function (Elsabahy and Wooley 2013). For example $\mathrm{ZnO}$ NPs tested in primary and immortalized immune cells were observed to release $\mathrm{Zn}^{2+}$ from the $\mathrm{ZnO}$ NPs which triggered the production of ROS, resulting in autophagic death of immune cells (Johnson et al. 2015). Intravenous administration of Ag NPs to rats in a 28-day repeated-dose toxicity study induces suppression of the functional immune system, as observed by a reduction in KHL-specific IgG and a reduced thymus weight (Vandebriel 
et al. 2014). Further, NPs have been reported to attract macrophages and neutrophils that in response can produce ROS (Krug et al. 2011). Activation of cytokine production, such as TNF- $\alpha$, by macrophages upon exposure to NPs may lead to inflammatory reactions.

In this thesis, effects of NPs on RAW264.7 cells were characterized, assessing cell viability, mitochondria related parameters and release of TNF- $\alpha$. RAW264.7 cells are macrophages and can be considered an in vitro model for this important cell type in the innate immune function. Focus was on mitochondria-related parameters, as previous research indicated that mitochondria play an important role in NP-induced toxicity and ROS production (Shvedova et al. 2012, Bhattacharjee et al. 2013). The NPs chosen for these studies were the metal oxide NPs. Endpoints studied included cell viability, oxidative stress, mitochondrial permeability transition pore (MPTP) opening, ATP levels and TNF- $\alpha$ production. The MPTP opening refers to a change in the inner mitochondrial membrane permeability (Bonora et al. 2014). An increase in MPTP opening results in a rapid loss of mitochondrial membrane potential, a decrease in ATP production and finally in the loss of cell integrity (Ly et al. 2003). The production of ROS within cells (oxidative stress) can be a main cause leading to cell death (Elsaesser et al. 2012). The NPs' ability to generate oxidative stress can be due to their high surface reactivity (Krug et al. 2011), the presence of transition metal ions in the case of metal oxides (Kim et al. 2014), or the fact that they can interact with the mitochondrial membrane and disrupt the electron transport chain (Bhattacharjee et al. 2012).

\section{PHYSICOCHEMICAL CHARACTERIZATION}

It is important to note that the assessment of toxicokinetics and-dynamics of NPs using in vitro assays is more complex than with regular chemicals. Factors like size, shape, composition, surface modifications, surface charge, interactions with salts, proteins and other macromolecules can have a major influence on the kinetics and dynamics of NPs (Oberdorster et al. 2005, Bouwmeester et al. 2011, Landsiedel et al. 2012). Surface charge has an effect on the potential for aggregation and agglomeration of the NPs, influencing the uptake and translocation of NPs by organisms (Graf et al. 2012). Due to their high surface energy, NPs adsorb biomolecules upon contact with biological environments, forming the so-called protein corona (Cedervall et al. 2007). The formation of the protein corona can influence the biodistribution and clearance (toxicokinetics) of NPs as well as their possible interactions with cellular macromolecules and cells, indicating that it can also influence their toxicity and pathophysiology (Meissner et al. 2009, Tenzer et al. 2013).

Not only the NP material, size and surface properties have been shown to play a role in determining the composition of the protein corona, also exposure time and the type of physiological fluid (e.g. human or murine, plasma or serum) are critical aspects (Fadeel 2012, 
Westmeier et al. 2016). This implies that when developing and validating possible in vitro alternative model systems for testing kinetics and dynamics of NPs, their characterisation in the actual medium or matrix in which they are tested and the relevance of this medium for the in vivo situation is of utmost importance (Gunsolus et al. 2016). Therefore, in the present thesis, the NPs were characterized in the medium used for the respective assay using dynamic light scattering (DLS), zeta potential and scanning electron microscopic (SEM) techniques.

\section{OUTLINE OF THE THESIS}

As outlined above the aim of the present thesis was to investigate the potential of in vitro testing strategies to detect hazards of NPs, focusing on toxicokinetic as well as toxicodynamic endpoints. Model NPs used for the studies included PS-NPs with different surface charges, and metal (oxide) NPs, of which some are possible constituents of specific food additives. The work is presented in seven chapters. The present chapter, chapter $\mathbf{1}$, provides an introduction and background information on the work discussed in this thesis.

Chapter 2 presents a review about the performance of in vitro models for studies on toxicokinetics of NPs. The chapter describes models that mimic the different barriers of the human body, with a focus on the lung, gut, skin and placental barrier, thus providing an overview of the state-of-the-art of using in vitro model systems to characterize the translocation of NPs across these different barriers.

In chapter 3 the BeWo b30 model for placental transfer was selected to study the role of NP charge on their placental translocation, using PS-NPs with positive and negative charge. The BeWo b30 model was used to get an initial qualitative impression about the capacity of PS-NPs to translocate across the placental barrier, which can be used to define priorities for further in vivo studies on placental translocation of NPs and developmental toxicity.

Chapter 4 investigated whether the same PS-NPs as tested in chapter 3 for placental translocation, are able to cause in vitro developmental toxicity in the ES-D3 cell differentiation assay of the EST. This study also focussed on the effect that charge may have on the in vitro developmental toxicity. Combining the results from Chapter 3 and 4 allows the integration of kinetic considerations with the in vitro developmental toxicity assay to improve the prediction of the in vivo hazards as done previously by Li et al. (Li et al. 2015, Li et al. 2015) for a series of azoles.

In chapter 5, toxic effects of a series of metal (oxide) NPs were tested in macrophage RAW264.7 cells in order to obtain insight in the effect of these NPs on cells that present a model for the innate immune response. In these macrophage RAW264.7 cells the effects of the metal (oxide) 
NPs was characterized on cell viability, TNF- $\alpha$ production and mitochondria-related parameters like formation of reactive oxygen species (ROS), mitochondrial permeability transition pore (MPTP) opening, and intracellular ATP, since previous research has indicated that mitochondria play an important role in NP-induced toxicity (Park et al. 2008, Sharma et al. 2011, Shvedova et al. 2012, Bhattacharjee et al. 2013).

In chapter 6 we used the in vitro RAW264.7 model to characterize the effect of surface charge and size of NPs in this in vitro model system using Ag NPs with two different sizes (20 and $50 \mathrm{~nm}$ ) and with three different surface coatings (chitosan, bovine serum albumin, polyvinylpyrrolidone). The effects of these Ag NPs on cell viability, TNF- $\alpha$ production, formation of ROS, mitochondrial permeability transition pore (MPTP) opening, and intracellular ATP were measured. All together the results of the work presented in this thesis will contribute to the further development and use of in vitro testing strategies for the safety testing of NPs and provide insight into selected potential hazards of the tested NPs.

Chapter 7 discusses the findings described in the thesis and presents suggestions for future research. 


\section{REFERENCES}

(2011). "Regulation EU no 1169/2011 on the provision of food information to consumers. Official Journal of the European Union. ." Official Journal of the European Union from http://eur-lex.europa.eu/legal-content/EN/ TXT/PDF/?uri=CELEX:32011R1169\&from=EN

Abdullah, M. M., F. M. Rajab and S. M. Al-Abbas (2014). "Structural and optical characterization of Cr2O3 nanostructures: Evaluation of its dielectric properties.” AIP Advances.

Ada, K., M. Turk, S. Oguztuzun, M. Kilic, M. Demirel, N. Tandogan, E. Ersayar and O. Latif (2010). “Cytotoxicity and apoptotic effects of nickel oxide nanoparticles in cultured HeLa cells." Folia histochemica et cytobiologica / Polish Academy of Sciences, Polish Histochemical and Cytochemical Society 48(4): 524-529.

Ahamed, M., M. J. Akhtar, H. A. Alhadlaq and S. A. Alrokayan (2015). "Assessment of the lung toxicity of copper oxide nanoparticles: current status." Nanomedicine (Lond) 10(15): 2365-2377.

Alarifi, S., D. Ali and S. Alkahtani (2016). "Mechanistic investigation of toxicity of chromium oxide nanoparticles in murine fibrosarcoma cells." Int J Nanomedicine 11: 1253-1259.

Ali, H., I. Kalashnikova, M. A. White, M. Sherman and E. Rytting (2013). "Preparation, characterization, and transport of dexamethasone-loaded polymeric nanoparticles across a human placental in vitro model.” Int J Pharm 454(1): 149-157.

Alili, L., S. Chapiro, G. U. Marten, A. M. Schmidt, K. Zanger and P. Brenneisen (2015). "Effect of Fe3O4 Nanoparticles on Skin Tumor Cells and Dermal Fibroblasts." Biomed Res Int 2015: 530957.

Arora, A. and G. W. Padua (2010). "Review: nanocomposites in food packaging." J Food Sci 75(1): R43-49.

Asharani, P. V., M. P. Hande and S. Valiyaveettil (2009). "Anti-proliferative activity of silver nanoparticles." BMC cell biology 10: 65 .

Athinarayanan, J., V. S. Periasamy, M. A. Alsaif, A. A. Al-Warthan and A. A. Alshatwi (2014). "Presence of nanosilica (E551) in commercial food products: TNF-mediated oxidative stress and altered cell cycle progression in human lung fibroblast cells." Cell biology and toxicology 30(2): 89-100.

Auffan, M., J. Rose, J. Y. Bottero, G. V. Lowry, J. P. Jolivet and M. R. Wiesner (2009). “Towards a definition of inorganic nanoparticles from an environmental, health and safety perspective." Nat Nanotechnol 4(10): 634-641.

Augustine-Rauch, K., C. X. Zhang and J. M. Panzica-Kelly (2010). "In vitro developmental toxicology assays: A review of the state of the science of rodent and zebrafish whole embryo culture and embryonic stem cell assays." Birth defects research. Part C, Embryo today : reviews 90(2): 87-98.

Baetke, S. C., T. Lammers and F. Kiessling (2015). "Applications of nanoparticles for diagnosis and therapy of cancer." The British journal of radiology 88(1054): 20150207.

Bhattacharjee, S., L. H. de Haan, N. M. Evers, X. Jiang, A. T. Marcelis, H. Zuilhof, I. M. Rietjens and G. M. Alink (2010). "Role of surface charge and oxidative stress in cytotoxicity of organic monolayer-coated silicon nanoparticles towards macrophage NR8383 cells." Part Fibre Toxicol 7: 25.

Bhattacharjee, S., D. Ershov, K. Fytianos, J. Gucht, G. Alink, I. Rietjens, A. Marcelis and H. Zuilhof (2012). "Cytotoxicity and cellular uptake of tri-block copolymer nanoparticles with different size and surface characteristics." Particle and Fibre Toxicology 9(1): 11.

Bhattacharjee, S., I. M. Rietjens, M. P. Singh, T. M. Atkins, T. K. Purkait, Z. Xu, S. Regli, A. Shukaliak, R. J. Clark, B. S. Mitchell, G. M. Alink, A. T. Marcelis, M. J. Fink, J. G. Veinot, S. M. Kauzlarich and H. Zuilhof (2013). "Cytotoxicity of surface-functionalized silicon and germanium nanoparticles: the dominant role of surface charges." Nanoscale 5(11): 4870-4883.

Blazquez, A. G., O. Briz, E. Gonzalez-Sanchez, M. J. Perez, C. I. Ghanem and J. J. Marin (2014). "The effect of acetaminophen on the expression of BCRP in trophoblast cells impairs the placental barrier to bile acids during maternal cholestasis." Toxicol Appl Pharmacol 277(1): 77-85.

Bonora, M., J. M. Bravo-San Pedro, G. Kroemer, L. Galluzzi and P. Pinton (2014). "Novel insights into the mitochondrial permeability transition." Cell cycle 13(17): 2666-2670.

Boudreau, M. D., M. S. Imam, A. M. Paredes, M. S. Bryant, C. K. Cunningham, R. P. Felton, M. Y. Jones, K. J. Davis and G. R. Olson (2016). "Differential Effects of Silver Nanoparticles and Silver Ions on Tissue Accumulation, Distribution, and Toxicity in the Sprague Dawley Rat Following Daily Oral Gavage Administration for 13 Weeks." Toxicol Sci 150(1): 131-160. 
Bouwmeester, H., I. Lynch, H. J. Marvin, K. A. Dawson, M. Berges, D. Braguer, H. J. Byrne, A. Casey, G. Chambers, M. J. Clift, G. Elia, T. F. Fernandes, L. B. Fjellsbo, P. Hatto, L. Juillerat, C. Klein, W. G. Kreyling, C. Nickel, M. Riediker and V. Stone (2011). "Minimal analytical characterization of engineered nanomaterials needed for hazard assessment in biological matrices." Nanotoxicology 5(1): 1-11.

Buerki-Thurnherr, T., M. von and P. Wick (2012). "Knocking at the door of the unborn child: engineered nanoparticles at the human placental barrier." Swiss medical weekly 142: 0.

Cartwright, L., M. S. Poulsen, H. M. Nielsen, G. Pojana, L. E. Knudsen, M. Saunders and E. Rytting (2012). "In vitro placental model optimization for nanoparticle transport studies." Int J Nanomedicine 7: 497-510.

Cedervall, T., I. Lynch, S. Lindman, T. Berggard, E. Thulin, H. Nilsson, K. A. Dawson and S. Linse (2007). "Understanding the nanoparticle-protein corona using methods to quantify exchange rates and affinities of proteins for nanoparticles." Proc Natl Acad Sci U S A 104(7): 2050-2055.

Chattopadhyay, S., S. K. Dash, S. Tripathy, B. Das, D. Mandal, P. Pramanik and S. Roy (2015). "Toxicity of cobalt oxide nanoparticles to normal cells; an in vitro and in vivo study." Chem Biol Interact 226: 58-71.

Chaudhry, Q., M. Scotter, J. Blackburn, B. Ross, A. Boxall, L. Castle, R. Aitken and R. Watkins (2008). "Applications and implications of nanotechnologies for the food sector." Food Addit Contam Part A Chem Anal Control Expo Risk Assess 25(3): 241-258.

Chiu, H. W., T. Xia, Y. H. Lee, C. W. Chen, J. C. Tsai and Y. J. Wang (2015). "Cationic polystyrene nanospheres induce autophagic cell death through the induction of endoplasmic reticulum stress." Nanoscale 7(2): 736-746.

Correia Carreira, S., L. Walker, K. Paul and M. Saunders (2013). “The toxicity, transport and uptake of nanoparticles in the in vitro BeWo b30 placental cell barrier model used within NanoTEST.” Nanotoxicology.

Dang, T., T. Le, T. Hoang and T. Mai (2015). "Synthesis of nanostructured manganese oxides based materials and application for supercapacitor." Advances in Natural Sciences; Nanoscience and Nanotechnology 6.

de Jong, E., M. Barenys, S. A. B. Hermsen, A. Verhoef, B. C. Ossendorp, J. G. M. Bessems and A. H. Piersma (2011). "Comparison of the mouse Embryonic Stem cell Test, the rat Whole Embryo Culture and the Zebrafish Embryotoxicity Test as alternative methods for developmental toxicity testing of six 1,2,4-triazoles." Toxicology and Applied Pharmacology 253(2): 103-111.

Dekkers, S., H. Bouwmeester, P. M. Bos, R. J. Peters, A. G. Rietveld and A. G. Oomen (2013). "Knowledge gaps in risk assessment of nanosilica in food: evaluation of the dissolution and toxicity of different forms of silica." Nanotoxicology 7(4): 367-377.

Dekkers, S., P. Krystek, R. J. Peters, D. P. Lankveld, B. G. Bokkers, P. H. van Hoeven-Arentzen, H. Bouwmeester and A. G. Oomen (2011). "Presence and risks of nanosilica in food products." Nanotoxicology 5(3): 393-405.

Deville, S., R. Penjweini, N. Smisdom, K. Notelaers, I. Nelissen, J. Hooyberghs and M. Ameloot (2015). "Intracellular dynamics and fate of polystyrene nanoparticles in A549 Lung epithelial cells monitored by image (cross-) correlation spectroscopy and single particle tracking." Biochim Biophys Acta 1853(10 Pt A): 2411-2419.

Dhawan, A. and V. Sharma (2010). "Toxicity assessment of nanomaterials: methods and challenges." Analytical and Bioanalytical Chemistry 398(2): 589-605.

Di Guglielmo, C., D. R. Lopez, J. De Lapuente, J. M. Mallafre and M. B. Suarez (2010). "Embryotoxicity of cobalt ferrite and gold nanoparticles: a first in vitro approach." Reprod Toxicol 30(2): 271-276.

Duran, N., C. P. Silveira, M. Duran and D. S. Martinez (2015). "Silver nanoparticle protein corona and toxicity: a mini-review." Journal of nanobiotechnology 13: 55.

ECVAM. (2012). "INVITTOX Protocols No. 113 Embryonic Stem Cell Test.” Retrieved August 26, 2013, from http://ecvam-dbalm.jrc.ec.europa.eu/public_view_doc.cfm?id=DC5ABDF7AC30F1B7DF7EF27E87D68AAC7180BB0BC12CB10496CDA74B54630A05A3291B895581F634.

Elsabahy, M. and K. L. Wooley (2013). "Cytokines as biomarkers of nanoparticle immunotoxicity." Chemical Society reviews 42(12): 5552-5576.

Elsaesser, A. and C. V. Howard (2012). “Toxicology of nanoparticles.” Adv Drug Deliv Rev 64(2): 129-137.

Ema, M., K. S. Hougaard, A. Kishimoto and K. Honda (2016). "Reproductive and developmental toxicity of carbon-based nanomaterials: A literature review." Nanotoxicology 10(4): 391-412.

Fadeel, B. (2012). "Clear and present danger? Engineered nanoparticles and the immune system." Swiss medical weekly 142: w13609.

Faust, J. J., K. Doudrick, Y. Yang, P. Westerhoff and D. G. Capco (2014). "Food grade titanium dioxide disrupts intestinal brush border microvilli in vitro independent of sedimentation." Cell biology and toxicology 30(3): 169-188. 
Foldbjerg, R., P. Olesen, M. Hougaard, D. A. Dang, H. J. Hoffmann and H. Autrup (2009). "PVP-coated silver nanoparticles and silver ions induce reactive oxygen species, apoptosis and necrosis in THP-1 monocytes." Toxicol Lett 190(2): 156-162.

Fort, D. J. and R. R. Paul (2002). "Enhancing the predictive validity of Frog Embryo Teratogenesis Assay--Xenopus (FETAX)." Journal of applied toxicology : JAT 22(3): 185-191.

Forte, M., G. Iachetta, M. Tussellino, R. Carotenuto, M. Prisco, M. De Falco, V. Laforgia and S. Valiante (2016). "Polystyrene nanoparticles internalization in human gastric adenocarcinoma cells.” Toxicol In Vitro 31: 126-136.

Fourches, D., D. Pu, C. Tassa, R. Weissleder, S. Y. Shaw, R. J. Mumper and A. Tropsha (2010). "Quantitative nanostructure-activity relationship modeling." ACS Nano 4(10): 5703-5712.

Frohlich, E. (2012). “The role of surface charge in cellular uptake and cytotoxicity of medical nanoparticles." Int J Nanomedicine 7: 5577-5591.

Garcia, T., D. Lafuente, J. Blanco, D. J. Sanchez, J. J. Sirvent, J. L. Domingo and M. Gomez (2016). "Oral subchronic exposure to silver nanoparticles in rats." Food Chem Toxicol 92: 177-187.

Genschow, E., H. Spielmann, G. Scholz, A. Seiler, N. Brown, A. Piersma, M. Brady, N. Clemann, H. Huuskonen, F. Paillard, S. Bremer and K. Becker (2002). "The ECVAM international validation study on in vitro embryotoxicity tests: results of the definitive phase and evaluation of prediction models. European Centre for the Validation of Alternative Methods." Alternatives to laboratory animals : ATLA 30(2): 151-176.

George, I., S. Vranic, S. Boland, A. Courtois and A. Baeza-Squiban (2015). "Development of an in vitro model of human bronchial epithelial barrier to study nanoparticle translocation.” Toxicol In Vitro 29(1): 51-58.

Gerloff, K., I. Fenoglio, E. Carella, J. Kolling, C. Albrecht, A. W. Boots, I. Forster and R. P. Schins (2012). "Distinctive toxicity of TiO2 rutile/anatase mixed phase nanoparticles on Caco-2 cells." Chemical research in toxicology 25(3): 646-655.

Geys, J., L. Coenegrachts, J. Vercammen, Y. Engelborghs, A. Nemmar, B. Nemery and P. H. Hoet (2006). "In vitro study of the pulmonary translocation of nanoparticles: a preliminary study." Toxicol Lett 160(3): 218-226.

Glista-Baker, E. E., A. J. Taylor, B. C. Sayers, E. A. Thompson and J. C. Bonner (2014). "Nickel nanoparticles cause exaggerated lung and airway remodeling in mice lacking the T-box transcription factor, TBX21 (T-bet)." Part Fibre Toxicol 11: 7.

Graf, C., Q. Gao, I. Schutz, C. N. Noufele, W. Ruan, U. Posselt, E. Korotianskiy, D. Nordmeyer, F. Rancan, S. Hadam, A. Vogt, J. Lademann, V. Haucke and E. Ruhl (2012). "Surface functionalization of silica nanoparticles supports colloidal stability in physiological media and facilitates internalization in cells." Langmuir 28(20): 7598-7613.

Grande, F. and P. Tucci (2016). “Titanium Dioxide Nanoparticles: a Risk for Human Health?” Mini reviews in medicinal chemistry 16(9): 762-769.

Griffith, R. D., B. J. Simmons, M. A. Yazdani Abyaneh, F. N. Bray, L. A. Falto-Aizpurua and K. Nouri (2015). "Colloidal Silver: Dangerous and Readily Available." JAMA dermatology 151(6): 667-668.

Gunsolus, I. L. and C. L. Haynes (2016). “Analytical Aspects of Nanotoxicology." Anal Chem 88(1): 451-479.

Horie, M., H. Fukui, K. Nishio, S. Endoh, H. Kato, K. Fujita, A. Miyauchi, A. Nakamura, M. Shichiri, N. Ishida, S. Kinugasa, Y. Morimoto, E. Niki, Y. Yoshida and H. Iwahashi (2011). "Evaluation of acute oxidative stress induced by $\mathrm{NiO}$ nanoparticles in vivo and in vitro." Journal of occupational health 53(2): 64-74.

Hougaard, K. S., L. Campagnolo, P. Chavatte-Palmer, A. Tarrade, D. Rousseau-Ralliard, S. Valentino, M. V. Park, W. H. de Jong, G. Wolterink, A. H. Piersma, B. L. Ross, G. R. Hutchison, J. S. Hansen, U. Vogel, P. Jackson, R. Slama, A. Pietroiusti and F. R. Cassee (2015). "A perspective on the developmental toxicity of inhaled nanoparticles." Reprod Toxicol 56: 118-140.

Huk, A., E. Izak-Nau, B. Reidy, M. Boyles, A. Duschl, I. Lynch and M. Dusinska (2014). "Is the toxic potential of nanosilver dependent on its size?" Part Fibre Toxicol 11: 65.

Hussain, S. M., D. B. Warheit, S. P. Ng, K. K. Comfort, C. M. Grabinski and L. K. Braydich-Stolle (2015). "At the Crossroads of Nanotoxicology in vitro: Past Achievements and Current Challenges." Toxicol Sci 147(1): $5-16$.

Ikeda, K., N. Utoguchi, H. Tsutsui, S. Yamaue, M. Homemoto, E. Nakao, Y. Hukunaga, K. Yamasaki, M. Myotoku and Y. Hirotani (2011). "In vitro approaches to evaluate placental drug transport by using differentiating JEG-3 human choriocarcinoma cells." Basic \& clinical pharmacology \& toxicology 108(2): 138-145.

Jelinek, R., M. Peterka and Z. Rychter (1985). “Chick embryotoxicity screening test--130 substances tested.” Indian J Exp Biol 23(10): 588-595. 
Johnson, B. M., J. A. Fraietta, D. T. Gracias, J. L. Hope, C. J. Stairiker, P. R. Patel, Y. M. Mueller, M. D. McHugh, L. J. Jablonowski, M. A. Wheatley and P. D. Katsikis (2015). "Acute exposure to ZnO nanoparticles induces autophagic immune cell death." Nanotoxicology 9(6): 737-748.

Johnston, H. J., M. Semmler-Behnke, D. M. Brown, W. Kreyling, L. Tran and V. Stone (2010). "Evaluating the uptake and intracellular fate of polystyrene nanoparticles by primary and hepatocyte cell lines in vitro." Toxicol Appl Pharmacol 242(1): 66-78.

Juch, H., L. Nikitina, P. Debbage, G. Dohr and M. Gauster (2013). "Nanomaterial interference with early human placenta: Sophisticated matter meets sophisticated tissues." Reproductive Toxicology 41(0): 73-79.

Kango S., K. S., Celli A., Njuguna J., Habibi Y., Kumar R. (2013). "Surface modification of inorganic nanoparticles for development of organic-inorganic nanocomposites-A review " Progress in Polymer Science 38: 12321261.

Karlsson, H. L., P. Cronholm, J. Gustafsson and L. Moller (2008). "Copper oxide nanoparticles are highly toxic: a comparison between metal oxide nanoparticles and carbon nanotubes." Chemical research in toxicology 21(9): 1726-1732.

Karlsson, H. L., J. Gustafsson, P. Cronholm and L. Moller (2009). "Size-dependent toxicity of metal oxide particles--a comparison between nano- and micrometer size." Toxicol Lett 188(2): 112-118.

Kawanishi, S., S. Oikawa, S. Inoue and K. Nishino (2002). "Distinct mechanisms of oxidative DNA damage induced by carcinogenic nickel subsulfide and nickel oxides." Environ Health Perspect 110 Suppl 5: 789-791.

Kim, C. S., H. D. Nguyen, R. M. Ignacio, J. H. Kim, H. C. Cho, E. H. Maeng, Y. R. Kim, M. K. Kim, B. K. Park and S. K. Kim (2014). "Immunotoxicity of zinc oxide nanoparticles with different size and electrostatic charge." Int J Nanomedicine 9 Suppl 2: 195-205.

Kim, Y., S. D. Kong, L. H. Chen, T. R. Pisanic, 2nd, S. Jin and V. I. Shubayev (2013). "In vivo nanoneurotoxicity screening using oxidative stress and neuroinflammation paradigms." Nanomedicine 9(7): 1057-1066.

Krug, H. F. and P. Wick (2011). "Nanotoxicology: An Interdisciplinary Challenge." Angew Chem Int Ed Engl.

Landsiedel, R., E. Fabian, L. Ma-Hock, W. Wohlleben, K. Wiench, F. Oesch and B. van Ravenzwaay (2012). “Toxico-/ biokinetics of nanomaterials." Arch Toxicol.

Lee, J. A., M. K. Kim, H. J. Paek, Y. R. Kim, M. K. Kim, J. K. Lee, J. Jeong and S. J. Choi (2014). “Tissue distribution and excretion kinetics of orally administered silica nanoparticles in rats.” Int J Nanomedicine 9 Suppl 2: 251-260.

Lee, J. Y., J. S. Kim, H. J. Cho and D. D. Kim (2014). "Poly(styrene)-b-poly(DL-lactide) copolymer-based nanoparticles for anticancer drug delivery." Int J Nanomedicine 9: 2803-2813.

Li, H., B. Flick, I. M. Rietjens, J. Louisse, S. Schneider and B. van Ravenzwaay (2015). "Extended evaluation on the ES-D3 cell differentiation assay combined with the BeWo transport model, to predict relative developmental toxicity of triazole compounds." Arch Toxicol.

Li, H., I. M. Rietjens, J. Louisse, M. Blok, X. Wang, L. Snijders and B. van Ravenzwaay (2015). "Use of the ES-D3 cell differentiation assay, combined with the BeWo transport model, to predict relative in vivo developmental toxicity of antifungal compounds." Toxicol In Vitro 29(2): 320-328.

Liu, R., H. Y. Zhang, Z. X. Ji, R. Rallo, T. Xia, C. H. Chang, A. Nel and Y. Cohen (2013). "Development of structure-activity relationship for metal oxide nanoparticles.” Nanoscale 5(12): 5644-5653.

Liu, X. and J. Sun (2010). "Endothelial cells dysfunction induced by silica nanoparticles through oxidative stress via JNK/P53 and NF-kappaB pathways.” Biomaterials 31(32): 8198-8209.

Ly, J. D., D. R. Grubb and A. Lawen (2003). "The mitochondrial membrane potential (deltapsi(m)) in apoptosis; an update." Apoptosis 8(2): 115-128.

Ma, J., T. Chen, J. Mandelin, A. Ceponis, N. E. Miller, M. Hukkanen, G. F. Ma and Y. T. Konttinen (2003). "Regulation of macrophage activation." Cellular and molecular life sciences : CMLS 60(11): 2334-2346.

Macharia Theuri, S., J. Ngonyo and M. Wangari Kagai (2016). "Alternatives to the use of animals in research, education and testing." Altex 33(2): 183-185.

Mahler, G. J., M. B. Esch, E. Tako, T. L. Southard, S. D. Archer, R. P. Glahn and M. L. Shuler (2012). “Oral exposure to polystyrene nanoparticles affects iron absorption.” Nat Nanotechnol 7(4): 264-271.

Manley, S. W., H. Li and R. H. Mortimer (2005). "The BeWo choriocarcinoma cell line as a model of iodide transport by placenta." Placenta 26(5): 380-386.

McShan, D., P. C. Ray and H. Yu (2014). "Molecular toxicity mechanism of nanosilver." Journal of food and drug analysis 22(1): 116-127. 
Meenambika, R., S. Ramalingom and T. Chithambara Thanu (2014). "Structural and Morphological properties of $\mathrm{Cr} 2 \mathrm{O} 3$ Nanoparticles synthesized by novel solvent free method.” International Journal of Engineering Research and Applications 4(2): 20-23.

Meissner, T., A. Potthoff and V. Richter (2009). "Physico-chemical characterization in the light of toxicological effects." Inhal Toxicol 21 Suppl 1: 35-39.

Mitra, S., T. Keswani, N. Ghosh, S. Goswami, A. Datta, S. Das, S. Maity and A. Bhattacharyya (2013). "Copper induced immunotoxicity promote differential apoptotic pathways in spleen and thymus." Toxicology 306 : 74-84.

Monti, D. M., D. Guarnieri, G. Napolitano, R. Piccoli, P. Netti, S. Fusco and A. Arciello (2015). "Biocompatibility, uptake and endocytosis pathways of polystyrene nanoparticles in primary human renal epithelial cells." Journal of biotechnology 193: 3-10.

Montiel-Davalos, A., J. L. Ventura-Gallegos, E. Alfaro-Moreno, E. Soria-Castro, E. Garcia-Latorre, J. G. Cabanas-Moreno, M. del Pilar Ramos-Godinez and R. Lopez-Marure (2012). "TiO(2) nanoparticles induce dysfunction and activation of human endothelial cells.” Chemical research in toxicology 25(4): 920-930.

Morimoto, Y., A. Ogami, M. Todoroki, M. Yamamoto, M. Murakami, M. Hirohashi, T. Oyabu, T. Myojo, K. Nishi, C. Kadoya, S. Yamasaki, H. Nagatomo, K. Fujita, S. Endoh, K. Uchida, K. Yamamoto, N. Kobayashi, J. Nakanishi and I. Tanaka (2010). "Expression of inflammation-related cytokines following intratracheal instillation of nickel oxide nanoparticles." Nanotoxicology 4(2): 161-176.

Myllynen, P. K., M. J. Loughran, C. V. Howard, R. Sormunen, A. A. Walsh and K. H. Vahakangas (2008). "Kinetics of gold nanoparticles in the human placenta." Reprod Toxicol 26(2): 130-137.

Nanoparticle Products Inventory, P. “The project on emerging nanotechnologies." from http://www.nanotechproject. org/cpi/.

Negahdary, M., Z. Arefian, H. A. Dastjerdi and M. Ajdary (2015). “Toxic effects of Mn2O3 nanoparticles on rat testis and sex hormone." Journal of natural science, biology, and medicine 6(2): 335-339.

Nel, A., T. Xia, L. Madler and N. Li (2006). “Toxic potential of materials at the nanolevel." Science 311(5761): 622-627.

Nishi, K., Y. Morimoto, A. Ogami, M. Murakami, T. Myojo, T. Oyabu, C. Kadoya, M. Yamamoto, M. Todoroki, M. Hirohashi, S. Yamasaki, K. Fujita, S. Endo, K. Uchida, K. Yamamoto, J. Nakanishi and I. Tanaka (2009). “Expression of cytokine-induced neutrophil chemoattractant in rat lungs by intratracheal instillation of nickel oxide nanoparticles." Inhal Toxicol 21(12): 1030-1039.

Oberdorster, G., E. Oberdorster and J. Oberdorster (2005). "Nanotoxicology: an emerging discipline evolving from studies of ultrafine particles.” Environ Health Perspect 113(7): 823-839.

Oberdorster, G., V. Stone and K. Donaldson (2007). "Toxicology of nanoparticles: A historical perspective." Nanotoxicology 1(1): 2-25.

Ogami, A., Y. Morimoto, T. Myojo, T. Oyabu, M. Murakami, M. Todoroki, K. Nishi, C. Kadoya, M. Yamamoto and I. Tanaka (2009). "Pathological features of different sizes of nickel oxide following intratracheal instillation in rats." Inhal Toxicol 21(10): 812-818.

Oukarroum, A., L. Barhoumi, M. Samadani and D. Dewez (2015). “Toxic effects of nickel oxide bulk and nanoparticles on the aquatic plant Lemna gibba L.” Biomed Res Int 2015: 501326.

Papis, E., F. Rossi, M. Raspanti, I. Dalle-Donne, G. Colombo, A. Milzani, G. Bernardini and R. Gornati (2009). "Engineered cobalt oxide nanoparticles readily enter cells.” Toxicol Lett 189(3): 253-259.

Park, E. J., J. Yi, K. H. Chung, D. Y. Ryu, J. Choi and K. Park (2008). "Oxidative stress and apoptosis induced by titanium dioxide nanoparticles in cultured BEAS-2B cells.” Toxicol Lett 180(3): 222-229.

Park, M. V., W. Annema, A. Salvati, A. Lesniak, A. Elsaesser, C. Barnes, G. McKerr, C. V. Howard, I. Lynch, K. A. Dawson, A. H. Piersma and W. H. de Jong (2009). "In vitro developmental toxicity test detects inhibition of stem cell differentiation by silica nanoparticles." Toxicol Appl Pharmacol 240(1): 108-116.

Park, M. V., A. M. Neigh, J. P. Vermeulen, L. J. de la Fonteyne, H. W. Verharen, J. J. Briede, H. van Loveren and W. H. de Jong (2011). "The effect of particle size on the cytotoxicity, inflammation, developmental toxicity and genotoxicity of silver nanoparticles." Biomaterials 32(36): 9810-9817.

Park, M. V. D. Z., W. Annema, A. Salvati, A. Lesniak, A. Elsaesser, C. Barnes, G. McKerr, C. V. Howard, I. Lynch, K. A. Dawson, A. H. Piersma and W. H. de Jong (2009). "In vitro developmental toxicity test detects inhibition of stem cell differentiation by silica nanoparticles." Toxicology and Applied Pharmacology 240(1): 108-116. 
Periasamy, V. S., J. Athinarayanan, A. M. Al-Hadi, F. A. Juhaimi, M. H. Mahmoud and A. A. Alshatwi (2015). "Identification of titanium dioxide nanoparticles in food products: induce intracellular oxidative stress mediated by TNF and CYP1A genes in human lung fibroblast cells." Environ Toxicol Pharmacol 39(1): 176-186.

Perreault, F., S. P. Melegari, C. H. da Costa, A. L. de Oliveira Franco Rossetto, R. Popovic and W. G. Matias (2012). "Genotoxic effects of copper oxide nanoparticles in Neuro 2A cell cultures." Science of The Total Environment 441(0): 117-124.

Peters, R., E. Kramer, A. G. Oomen, Z. E. Rivera, G. Oegema, P. C. Tromp, R. Fokkink, A. Rietveld, H. J. Marvin, S. Weigel, A. A. Peijnenburg and H. Bouwmeester (2012). "Presence of nano-sized silica during in vitro digestion of foods containing silica as a food additive." ACS Nano 6(3): 2441-2451.

Peters, R. J., G. van Bemmel, Z. Herrera-Rivera, H. P. Helsper, H. J. Marvin, S. Weigel, P. C. Tromp, A. G. Oomen, A. G. Rietveld and H. Bouwmeester (2014). "Characterization of titanium dioxide nanoparticles in food products: analytical methods to define nanoparticles.” J Agric Food Chem 62(27): 6285-6293.

Phosphorex Commercial Applications Use Polystyrene Nanoparticles. 2013 ; Available from: http://www.phosphorex.com/1/post/2013/07/commercial-applications-use-polystyrene-nanoparticles.html.

Pietroiusti, A., M. Massimiani, I. Fenoglio, M. Colonna, F. Valentini, G. Palleschi, A. Camaioni, A. Magrini, G. Siracusa, A. Bergamaschi, A. Sgambato and L. Campagnolo (2011). "Low Doses of Pristine and Oxidized Single-Wall Carbon Nanotubes Affect Mammalian Embryonic Development.” ACS Nano 5(6): 4624-4633.

Poulsen, M. S., T. Mose, L. L. Maroun, L. Mathiesen, L. E. Knudsen and E. Rytting (2015). "Kinetics of silica nanoparticles in the human placenta." Nanotoxicology 9 Suppl 1: 79-86.

Powers, C. M., A. S. Bale, A. D. Kraft, S. L. Makris, J. Trecki, J. Cowden, A. Hotchkiss and P. A. Gillespie (2013). "Developmental neurotoxicity of engineered nanomaterials: identifying research needs to support human health risk assessment." Toxicol Sci 134(2): 225-242.

Prietl, B., C. Meindl, E. Roblegg, T. R. Pieber, G. Lanzer and E. Frohlich (2014). "Nano-sized and micro-sized polystyrene particles affect phagocyte function." Cell biology and toxicology 30(1): 1-16.

Prow, T. W., N. A. Monteiro-Riviere, A. O. Inman, J. E. Grice, X. Chen, X. Zhao, W. H. Sanchez, A. Gierden, M. A. Kendall, A. V. Zvyagin, D. Erdmann, J. E. Riviere and M. S. Roberts (2012). "Quantum dot penetration into viable human skin." Nanotoxicology 6(2): 173-185.

Rahman, M. F., J. Wang, T. A. Patterson, U. T. Saini, B. L. Robinson, G. D. Newport, R. C. Murdock, J. J. Schlager, S. M. Hussain and S. F. Ali (2009). "Expression of genes related to oxidative stress in the mouse brain after exposure to silver-25 nanoparticles." Toxicol Lett 187(1): 15-21.

Rajiv, S., J. Jerobin, V. Saranya, M. Nainawat, A. Sharma, P. Makwana, C. Gayathri, L. Bharath, M. Singh, M. Kumar, A. Mukherjee and N. Chandrasekaran (2016). "Comparative cytotoxicity and genotoxicity of cobalt (II, III) oxide, iron (III) oxide, silicon dioxide, and aluminum oxide nanoparticles on human lymphocytes in vitro." Human \& experimental toxicology 35(2): 170-183.

Recordati, C., M. De Maglie, S. Bianchessi, S. Argentiere, C. Cella, S. Mattiello, F. Cubadda, F. Aureli, M. D’Amato, A. Raggi, C. Lenardi, P. Milani and E. Scanziani (2016). "Tissue distribution and acute toxicity of silver after single intravenous administration in mice: nano-specific and size-dependent effects.” Part Fibre Toxicol 13: 12.

Salvati, A., C. Aberg, T. dos Santos, J. Varela, P. Pinto, I. Lynch and K. A. Dawson (2011). "Experimental and theoretical comparison of intracellular import of polymeric nanoparticles and small molecules: toward models of uptake kinetics." Nanomedicine 7(6): 818-826.

Saunders, M. (2009). “Transplacental transport of nanomaterials." Wiley Interdiscip Rev Nanomed Nanobiotechnol 1(6): 671-684.

Senapati, V. A., A. K. Jain, G. S. Gupta, A. K. Pandey and A. Dhawan (2015). “Chromium oxide nanoparticle-induced genotoxicity and p53-dependent apoptosis in human lung alveolar cells.” Journal of applied toxicology : JAT 35(10): 1179-1188.

Sharma, V., D. Anderson and A. Dhawan (2011). "Zinc oxide nanoparticles induce oxidative stress and genotoxicity in human liver cells (HepG2).” J Biomed Nanotechnol 7(1): 98-99.

Shi, H., R. Magaye, V. Castranova and J. Zhao (2013). “Titanium dioxide nanoparticles: a review of current toxicological data." Part Fibre Toxicol 10: 15.

Shi, M., L. Cheng, Z. Zhang, Z. Liu and X. Mao (2015). "Ferroferric oxide nanoparticles induce prosurvival autophagy in human blood cells by modulating the Beclin 1/Bcl-2/VPS34 complex.” Int J Nanomedicine 10: 207-216. 
Shimada, M., W. N. Wang, K. Okuyama, T. Myojo, T. Oyabu, Y. Morimoto, I. Tanaka, S. Endoh, K. Uchida, K. Ehara, H. Sakurai, K. Yamamoto and J. Nakanishi (2009). "Development and evaluation of an aerosol generation and supplying system for inhalation experiments of manufactured nanoparticles." Environ Sci Technol 43(14): 5529-5534.

Shvedova, A. A., A. Pietroiusti, B. Fadeel and V. E. Kagan (2012). "Mechanisms of carbon nanotube-induced toxicity: focus on oxidative stress." Toxicol Appl Pharmacol 261(2): 121-133.

Siddiqui, M. A., M. Ahamed, J. Ahmad, M. A. Majeed Khan, J. Musarrat, A. A. Al-Khedhairy and S. A. Alrokayan (2012). "Nickel oxide nanoparticles induce cytotoxicity, oxidative stress and apoptosis in cultured human cells that is abrogated by the dietary antioxidant curcumin." Food Chem Toxicol 50(3-4): 641-647.

Simon, P., Q. Chaudhry and D. Bakos (2008). "Migration of engineered nanoparticles from polymer packaging to food - a physicochemical view." J Food Nutr Res 47(3): 105-113.

Smith, P. D., L. E. Smythies, R. Shen, T. Greenwell-Wild, M. Gliozzi and S. M. Wahl (2011). "Intestinal macrophages and response to microbial encroachment." Mucosal immunology 4(1): 31-42.

Song, P., D. Wen, X. Guo and T. Korakianitis (2008). “Oxidation investigation of nickel nanoparticles.” physical chemistry chemical physics 10(33): 5057-5065.

Sonnegaard Poulsen, M., T. Mose, L. Leth Maroun, L. Mathiesen, L. Ehlert Knudsen and E. Rytting (2013). "Kinetics of silica nanoparticles in the human placenta." Nanotoxicology.

Spielmann, H., A. Seiler, S. Bremer, L. Hareng, T. Hartung, H. Ahr, E. Faustman, U. Haas, G. J. Moffat, H. Nau, P. Vanparys, A. Piersma, J. R. Sintes and J. Stuart (2006). “The practical application of three validated in vitro embryotoxicity tests. The report and recommendations of an ECVAM/ZEBET workshop (ECVAM workshop 57)." Alternatives to laboratory animals : ATLA 34(5): 527-538.

Stone, V., H. Johnston and R. P. Schins (2009). "Development of in vitro systems for nanotoxicology: methodological considerations." Crit Rev Toxicol 39(7): 613-626.

Su, C. L., T. T. Chen, C. C. Chang, K. J. Chuang, C. K. Wu, W. T. Liu, K. F. Ho, K. Y. Lee, S. C. Ho, H. E. Tseng, H. C. Chuang, T. J. Cheng and G. Taiwan CardioPulmonary Research (2013). "Comparative proteomics of inhaled silver nanoparticles in healthy and allergen provoked mice." International journal of nanomedicine 8: 2783-2799.

Tavares, K., A. Caloto-Oliveira, D. Vicentini, S. Melegari and W. Matias (2014). "Acute toxicity of copper and chromium oxide nanoparticles to Daphnia similis." Ecotoxicolo. Environ. Contam. 9(1): 43-50.

Tenzer, S., D. Docter, J. Kuharev, A. Musyanovych, V. Fetz, R. Hecht, F. Schlenk, D. Fischer, K. Kiouptsi, C. Reinhardt, K. Landfester, H. Schild, M. Maskos, S. K. Knauer and R. H. Stauber (2013). "Rapid formation of plasma protein corona critically affects nanoparticle pathophysiology.” Nat Nanotechnol.

Valdiglesias, V., N. Fernandez-Bertolez, G. Kilic, C. Costa, S. Costa, S. Fraga, M. J. Bessa, E. Pasaro, J. P. Teixeira and B. Laffon (2016). "Are iron oxide nanoparticles safe? Current knowledge and future perspectives." Journal of trace elements in medicine and biology : organ of the Society for Minerals and Trace Elements.

Van der Jagt, K., S. Munn, J. Torslov and J. de Bruijn (2004). "Alternative Approaches can reduce the use of test animals under REACH. Addendum to the report "assessment of additional testing needs under REACH. Effects of (Q)SARs, Risk Based Testing and Volunary Industry Initiatives.” JRC Report EUR: 25.

Vandebriel, R. J., E. C. Tonk, L. J. de la Fonteyne-Blankestijn, E. R. Gremmer, H. W. Verharen, L. T. van der Ven, H. van Loveren and W. H. de Jong (2014). "Immunotoxicity of silver nanoparticles in an intravenous 28-day repeated-dose toxicity study in rats." Part Fibre Toxicol 11: 21.

Varela, J. A., M. G. Bexiga, C. Aberg, J. C. Simpson and K. A. Dawson (2012). "Quantifying size-dependent interactions between fluorescently labeled polystyrene nanoparticles and mammalian cells." Journal of nanobiotechnology 10: 39.

Verstraelen, S., S. Remy, E. Casals, P. De Boever, H. Witters, A. Gatti, V. Puntes and I. Nelissen (2014). “Gene expression profiles reveal distinct immunological responses of cobalt and cerium dioxide nanoparticles in two in vitro lung epithelial cell models." Toxicol Lett 228(3): 157-169.

Walczak, A. P., E. Kramer, P. J. Hendriksen, R. Helsdingen, M. van der Zande, I. M. Rietjens and H. Bouwmeester (2015). "In vitro gastrointestinal digestion increases the translocation of polystyrene nanoparticles in an in vitro intestinal co-culture model." Nanotoxicology 9(7): 886-894.

Walczak, A. P., E. Kramer, P. J. Hendriksen, P. Tromp, J. P. Helsper, M. van der Zande, I. M. Rietjens and H. Bouwmeester (2014). "Translocation of differently sized and charged polystyrene nanoparticles in in vitro intestinal cell models of increasing complexity." Nanotoxicology: 1-9. 
Warheit, D. B., T. R. Webb, K. L. Reed, S. Frerichs and C. M. Sayes (2007). "Pulmonary toxicity study in rats with three forms of ultrafine-TiO2 particles: differential responses related to surface properties." Toxicology 230(1): 90-104.

Watson, C., J. Ge, J. Cohen, G. Pyrgiotakis, B. P. Engelward and P. Demokritou (2014). "High-throughput screening platform for engineered nanoparticle-mediated genotoxicity using CometChip technology." ACS Nano 8(3): 2118-2133.

Weir, A., P. Westerhoff, L. Fabricius, K. Hristovski and N. von Goetz (2012). “Titanium dioxide nanoparticles in food and personal care products.” Environ Sci Technol 46(4): 2242-2250.

Westmeier, D., R. H. Stauber and D. Docter (2016). "The concept of bio-corona in modulating the toxicity of engineered nanomaterials (ENM).” Toxicol Appl Pharmacol 299: 53-57.

Wick, P., A. Malek, P. Manser, D. Meili, X. Maeder-Althaus, L. Diener, P. A. Diener, A. Zisch, H. F. Krug and U. von Mandach (2010). "Barrier capacity of human placenta for nanosized materials." Environmental health perspectives 118(3): 432-436.

Winter, M., H. D. Beer, V. Hornung, U. Kramer, R. P. Schins and I. Forster (2011). "Activation of the inflammasome by amorphous silica and $\mathrm{TiO} 2$ nanoparticles in murine dendritic cells." Nanotoxicology 5(3): 326-340.

$\mathrm{Wu}$, J. and J. Sun (2011). "Investigation on mechanism of growth arrest induced by iron oxide nanoparticles in PC12 cells.” J Nanosci Nanotechnol 11(12): 11079-11083.

Wu, W., Q. He and C. Jiang (2008). "Magnetic iron oxide nanoparticles: synthesis and surface functionalization strategies.” Nanoscale research letters 3(11): 397-415.

Xia, T., M. Kovochich, M. Liong, J. I. Zink and A. E. Nel (2008). "Cationic polystyrene nanosphere toxicity depends on cell-specific endocytic and mitochondrial injury pathways." ACS Nano 2(1): 85-96.

Yacobi, N. R., L. Demaio, J. Xie, S. F. Hamm-Alvarez, Z. Borok, K. J. Kim and E. D. Crandall (2008). "Polystyrene nanoparticle trafficking across alveolar epithelium." Nanomedicine 4(2): 139-145.

Yamashita, K., Y. Yoshioka, K. Higashisaka, K. Mimura, Y. Morishita, M. Nozaki, T. Yoshida, T. Ogura, H. Nabeshi, K. Nagano, Y. Abe, H. Kamada, Y. Monobe, T. Imazawa, H. Aoshima, K. Shishido, Y. Kawai, T. Mayumi, S. Tsunoda, N. Itoh, T. Yoshikawa, I. Yanagihara, S. Saito and Y. Tsutsumi (2011). "Silica and titanium dioxide nanoparticles cause pregnancy complications in mice." Nat Nanotechnol 6(5): 321-328.

Ying, E. and H. M. Hwang (2010). "In vitro evaluation of the cytotoxicity of iron oxide nanoparticles with different coatings and different sizes in A3 human T lymphocytes." The Science of the total environment 408(20): 4475-4481.

Zhang, H., Z. Ji, T. Xia, H. Meng, C. Low-Kam, R. Liu, S. Pokhrel, S. Lin, X. Wang, Y. P. Liao, M. Wang, L. Li, R. Rallo, R. Damoiseaux, D. Telesca, L. Madler, Y. Cohen, J. I. Zink and A. E. Nel (2012). "Use of metal oxide nanoparticle band gap to develop a predictive paradigm for oxidative stress and acute pulmonary inflammation." ACS Nano 6(5): 4349-4368.

zur Nieden, N. I., G. Kempka and H. J. Ahr (2004). "Molecular multiple endpoint embryonic stem cell test--a possible approach to test for the teratogenic potential of compounds." Toxicol Appl Pharmacol 194(3): 257-269. 


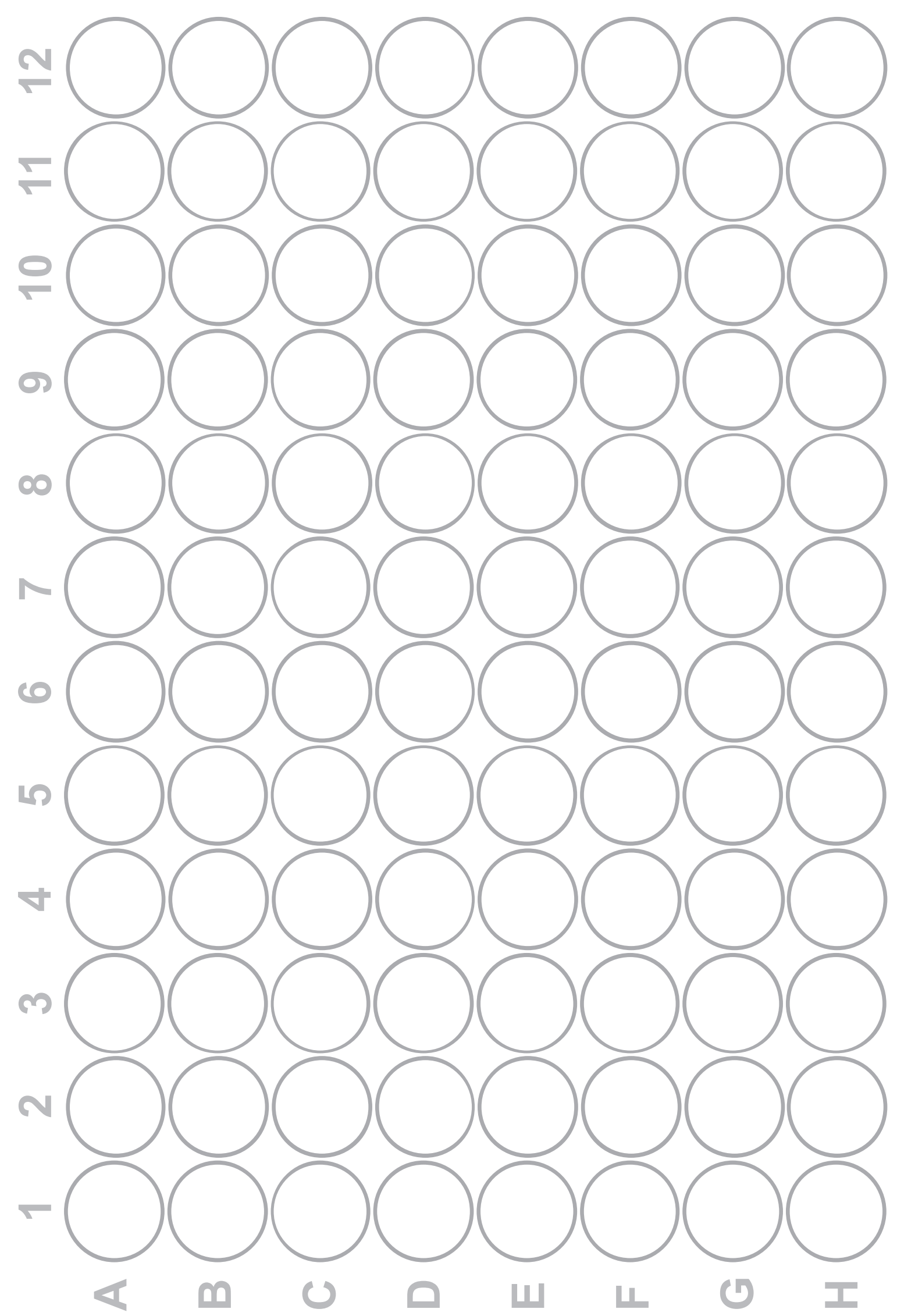




\section{Chapter 2}

\section{Progress and future of in vitro models to study translocation of nanoparticles}

Hedwig M. Braakhuis

Samantha K. Kloet

Sanja Kezic

Frieke Kuper

Margriet V. D. Z. Park

Susann Bellmann

Meike van der Zande

Séverine Le Gac

Petra Krystek

Ruud J. B. Peters

Ivonne M. C. M. Rietjens

Hans Bouwmeester

Based on: Archives of Toxicology (2015) 89(9):1469-95 


\section{ABSTRACT}

The increasing use of nanoparticles in products likely results in increased exposure of both workers and consumers. Because of their small size, there are concerns that nanoparticles unintentionally cross the barriers of the human body. Several in vivo rodent studies show that, dependent on the exposure route, time, and concentration, and their characteristics, nanoparticles can cross the lung, gut, skin, and placental barrier. This review aims to evaluate the performance of in vitro models that mimic the barriers of the human body, with a focus on the lung, gut, skin, and placental barrier. For these barriers, in vitro models of varying complexity are available, ranging from single- cell-type monolayer to multi-cell (3D) models. Only a few studies are available that allow comparison of the in vitro translocation to in vivo data. This situation could change since the availability of analytical detection techniques is no longer a limiting factor for this comparison. It was concluded that to further develop in vitro models to be used in risk assessment, the current strategy to improve the models to more closely mimic the human situation by using co-cultures of different cell types and microfluidic approaches to better control the tissue microenvironments are essential. At the current state of the art, the in vitro models do not yet allow prediction of absolute transfer rates but they do support the definition of relative transfer rates and can thus help to reduce animal testing by setting priorities for subsequent in vivo testing. 


\section{INTRODUCTION}

Nanoparticles have attractive and novel properties compared with their bulk counterparts and are therefore used in an increasing number of consumer products (Nanotechnologies 2014). Examples are zinc oxide and titanium dioxide nanoparticles in sunscreens and silver nanoparticles in food packaging material, textiles, and cosmetics, but many more have been identified (Bouwmeester et al. 2014). The increasing use of nanoparticles in products likely results in increasing exposure of both workers and consumers. Because of the unique properties of nanoparticles that are related to their small size, concerns arise that nanoparticles would unintentionally cross the barriers of the human body, which would result in internal exposure to nanoparticles potentially leading to adverse effects.

Several in vivo studies have been performed to assess the distribution of nanoparticles after inhalation, oral exposure, skin exposure, and intravenous injection (Balasubramanian et al. 2010, Braakhuis et al. 2014a, Creutzenberg et al. 2012, De Jong et al. 2008, Elder et al. 2006, Geraets et al. 2012, Kreyling et al. 2009, Leite-Silva et al. 2013, Ma-Hock et al. 2012, Oberdorster et al. 2004, Semmler et al. 2004, Takenaka et al. 2001, van der Zande et al. 2012, 2014). These studies show that, depending on the exposure route, time, concentration, as well as on their characteristics, nanoparticles can cross the lung, gut, skin, and placental barrier.

Information on the kinetics of nanoparticles in the human body is essential for risk assessment purposes, because of their potency to accumulate. The overall resultant of absorption, distribution, metabolism, and excretion (ADME), i.e. internal exposure, will determine target tissue doses and will be critical for the ultimate systemic adverse health effects (Geraets et al. 2014). Even in cases of low absorption of nanoparticles, the often chronic nature of the exposure (inhalatory, oral or dermal) might result in internal accumulation of the nanoparticles potentially reaching levels that might give rise to health concerns (van Kesteren et al. 2014). The current risk assessment of nanoparticles (and chemicals) mainly relies on in vivo studies using animal models (EFSA 2011). While these in vivo studies provide unique information on the distribution of nanoparticles in a whole organism, the number of animal studies should be reduced as much as possible for several reasons (Hartung et al. 2013). First, the use of animals is ethically debatable. Secondly, animal models do not fully simulate the physiology of humans. Lastly, given the great number of and variety in different nanoparticles, it is impossible and economically not feasible to test all of them through in vivo studies. Therefore, in vitro models have been developed to study the translocation of nanoparticles (Hartung et al. 2013) and estimate the in vivo internal exposure. However, before such in vitro models can reliably be used in risk assessment of nanoparticles, they need to be well described and validated (Kandarova and Letasiova 2011, Worth and Balls 2004) using in vivo data (Genschow et al. 2002). 
To obtain reliable NP kinetic data from in vitro or in vivo studies, robust analytical detection methods should be used in the experiments. Over the last couple of years, the quality of NP characterization methods used in kinetic studies has been improved, but much is to be gained here. Therefore, the current state of the knowledge on analytical detection methods was reviewed and directions for further improvement and incorporation in in vitro or in vivo studies were proposed.

This review aimed to evaluate the existence and performance of in vitro models that mimic the barriers of the human body. Where possible the observed translocation in vitro was compared to the in vivo translocation to compare to what extent the in vitro results mimic the in vivo situation. In addition, the placental barrier was included that protects the unborn foetus from exposure via the maternal circulation. For an overview on the status of alternatives for regulatory toxicology in general, we refer to the 2014 JRC Science and Policy Report by Worth et al. (2014). First the different in vitro models that are currently in use to study the transfer of nanoparticles via inhalation, oral uptake, skin uptake, and placental uptake were described. In vitro models that are used only to assess the toxicity of nanoparticles, but not to measure translocation, are excluded from this review. After defining the in vitro models available to study transfer across the different barriers, the results of the in vitro models were compared with available in vivo data and discuss their predictive value. Finally, recommendations were given for the future development of relevant in vitro models.

\section{Introduction to the lung barrier}

The main function of the lungs is to transport oxygen from the atmosphere into the bloodstream and to release carbon dioxide from the bloodstream into the atmosphere. During inhalation, air travels from the mouth or nose through the nasopharynx, oropharynx, larynx, and trachea. The trachea divides into two main bronchi, which branch to the left and right lungs and subsequently subdivide into a system of bronchi and bronchioles until the alveoli where the gas exchange takes place. The airways are lined by ciliated respiratory epithelium, which is covered by a mucus layer. The mucociliary movement is an important clearance mechanism, especially to remove inhaled (nano)particles. Deeper in the airways, the clearance is slower, given the increased pathway length and decreased mucous velocity (Geiser and Kreyling 2010). Nanoparticles, especially those that dissolve readily such as $\mathrm{ZnO}$, may be able to translocate the mucus layer and reach the epithelial cells and thus cause local damage (Frieke Kuper et al. 2015, Landsiedel et al.2014a, Vandebriel and De Jong 2012). They may also be able to cross the epithelial barrier and reach underlying interstitium with its blood and lymph vasculature.

The alveoli are lined by a single epithelial layer under which is an interstitium with extracellular matrix, blood capillaries, and stromal cells. The epithelial layer is covered by surfactant 
at the alveolar luminal side. Alveolar type I cells form the structure of the alveolar wall. These cells are very thin to improve the gas exchange. In addition, type I cells have tight junctions to prevent chemicals and particles from entering the bloodstream. Besides type I cells, alveolar type II cells secrete pulmonary surfactant to lower the surface tension. Finally, for clearance of particles and pathogens from the lungs, alveolar macrophages are present (Klein et al. 2011, Möller et al. 2010).

To cross the lung-blood barrier, nanoparticles must deposit in the alveolar region. The deposition of inhaled particles depends on the morphology of the lungs, the respiratory conditions, and the physicochemical properties of the particles. The most important physicochemical properties of inhaled particles that influence deposition are (agglomerate) size, size distribution, density, shape, charge, and hygroscopicity (Braakhuis et al. 2014b, Carvalho et al. 2011, Pilcer and Amighi 2010). When the agglomerate size of nanoparticles is $<100 \mathrm{~nm}$ but $>10 \mathrm{~nm}$, a considerable part will deposit in the alveolar region (about $30 \%$ of the particles) (Asgharian et al. 2009, ICRP 1994, Oberdorster 1989). Below $30 \mathrm{~nm}$, the deposition shifts from the alveoli more towards to tracheobronchial region (Braakhuis et al. 2014b).

Once deposited in the alveoli, nanoparticles can be cleared from the lungs by alveolar macrophages. However, single nanoparticles and agglomerates of $<100 \mathrm{~nm}$ are less efficiently phagocytized by alveolar macrophages compared with microparticles or large agglomerates of $>1 \mu \mathrm{m}$ (Bakand et al. 2012, Muhlfeld et al. 2008, Phalen et al. 2010). After uptake of the particles, macrophages can move gradually upward by the mucociliary escalator, are subsequently swallowed, and enter the gastrointestinal tract. If not cleared by phagocytosis, nanoparticles can be taken up by the alveolar epithelium and reach the pulmonary interstitium from which they are transported to the local lymph nodes, or reach the blood circulation (Borm et al. 2006). Translocated particles may subsequently reach organs where they can be taken up and might cause damage (Braakhuis et al. 2014b).

\section{Introduction to the intestinal barrier}

The primary functions of the human gastrointestinal (GI) tract are related to the digestion and absorption of nutrients and electrolytes, and to water homeostasis. The GI tract is responsive to internal stimuli as well as to (microbe) stimuli from the lumen content. The GI epithelial layer forms a tight, but selective barrier: nutrients are absorbed efficiently, while microbes, for example, are not. Anatomically the gut wall can be divided into the mucosa, submucosa, muscularis externa, and serosa. At the lumen site, the gut wall consists of a mucosa, which is a combined mucus and cell epithelial layer. The composition of the mucus and the type of cells is variable along the GI tract and reflects the specialized function of each region. The submucosa is a layer of connective tissue that contains lymphatic and blood vessels as well as ganglion and nerve cells. In the next layer, the muscularis externa, the main smooth muscles 
of the gut are found. The thickness of the muscle layer varies. The serosa is a squamous epithelium (mesothelium) which sits on connective tissue and is continuous with the abdominal peritoneum.

The small intestine is the site where most of the chemical and mechanical digestion takes place and where almost all of the absorption of nutrients and electrolytes is carried out. The wall of the small intestine is lined with absorptive mucosa. The mucosal surface is extended by the presence of crypts and villi. The most common epithelial cell is the enterocyte, its major function being to absorb nutrients. The second cell type is the mucus-secreting goblet cell, the mucus acts as a lubricant and protects the mucosa from irritation. Lastly, the gut-associated lymphoid tissue (GALT) includes several specialized cells including Peyer's patches, M cells, and intraepithelial lymphocytes, which are part of the intestinal immune system.

Following ingestion, translocation of particles into and across the gastrointestinal mucosa can occur via four different: (1) via endocytosis, through enterocytes, (2) via the M cell-rich layer of Peyer's patches (small intestinal lymphoid aggregates), (3) via persorption, where particles can trans- locate through a 'hole' left in the epithelium when enterocytes shed from the villous tip, and (4) via the paracellular route, where nanoparticles pass across tight junctions of the epithelial cell layer (Powell et al. 2010). While the exposure of the gastrointestinal mucosa to engineered nanoparticles might pose yet unresolved heath issues, it is important to realize that people in the Western world are daily exposed to sub-micrometre-sized mineral particles (Powell et al. 1996, 2010). These particles have been observed to be composed of aluminosilicates, titanium dioxide, and a small percentage of non-aluminium-containing silicates such as silica $\left(\mathrm{SiO}_{2}\right)$ and magnesium trisilicate (talc) (Dekkers et al. 2011, Powell et al. 1996).

\section{Introduction to the skin barrier}

The skin is a barrier towards loss of water and ingress of microorganisms, UV radiation, and potentially harmful chemicals. Although the permeability of skin is an order of magnitude less than that of the intestinal epithelial cell layer, due to its large surface of almost $2 \mathrm{~m}^{2}$ and likelihood of dermal exposure in everyday life, the skin can pose an important absorption route for nanoparticles. The barrier function of the skin is generally attributed to its upper layer, the stratum corneum. The stratum corneum consists of stacked layers of corneocytes, enucleated flattened cells that are surrounded by impermeable cornified envelope and embedded in organized lipid bilayers. There are three potential routes by which a penetrant can diffuse across the stratum corneum: (1) across the lipid bilayers (intercellular route; Fig. 1a), (2) across the corneocytes and lipid bilayers (intracellular route; Fig. 1b), and (3) along hair follicles and sweat glands (Fig. 1c). 


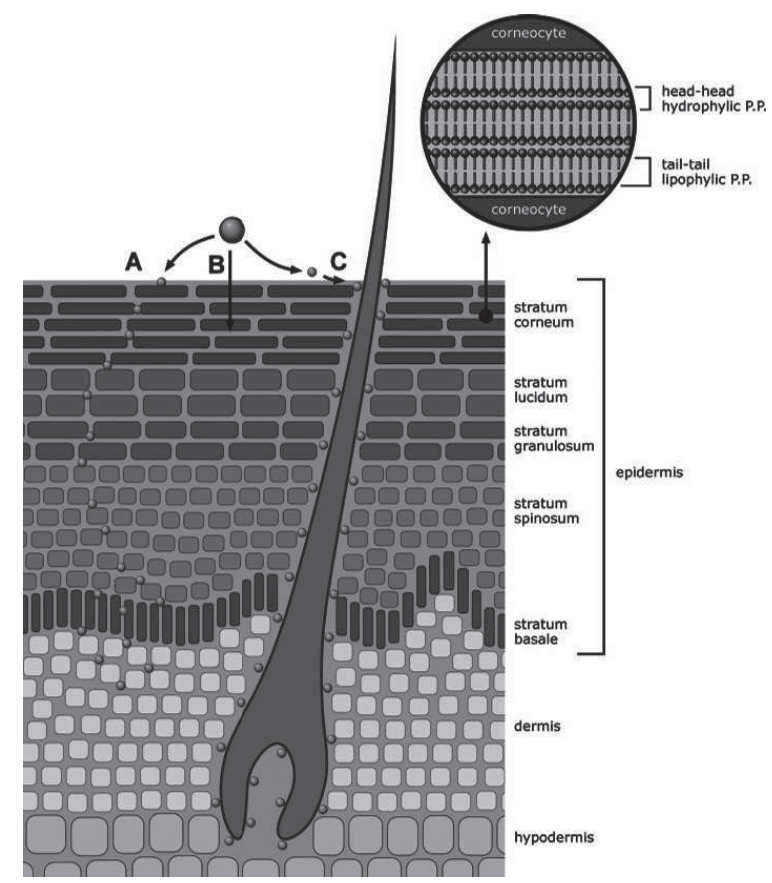

Figure 1. A schematic illustration of the skin and main penetration routes, insert showing the lipid bilayers between corneocytes. Route A: across the lipid bilayers (intercellular route); Route B: across the corneocytes and lipid bilayers (intracellular route); Route C: along hair follicles and sweat glands

The intracellular route is thermodynamically unfavourable due to the highly impermeable cornified envelope of the corneocytes. For most chemicals, the route across lipid bilayers represents the main diffusional pathway. The stratum corneum lipids are organized in two coexisting lamellar phases: a long periodicity phase with a repeat distance of around $13 \mathrm{~nm}$ and a short periodicity phase with a repeat distance of around $6 \mathrm{~nm}$ (Baroli 2010). The space between the tail-tail and head-head domains of the lipid bilayers (Fig. 1) restricts the size of a NP that is able to penetrate across the stratum corneum (Baroli 2010, Cevc and Vierl 2010). Another restriction factor for the penetration of nanoparticles across the intracellular route is high diffusion resistance for particles larger than $5 \mathrm{~nm}$ (Watkinson et al. 2013). Thus, theoretically for nanoparticles larger than approximately $5 \mathrm{~nm}$ the route along hair follicles might represent the predominant penetration route.

This view, however, can be challenged in the case of a compromised skin barrier, which can occur due to intrinsic and environmental factors. For instance, one of the main features of a common inflammatory skin disease, atopic dermatitis (AD), is a damaged skin barrier (Kezic et al.2014). Furthermore, exposure to skin irritating chemicals such as detergents and or- 
ganic solvents in the work place as well as in everyday life can also lead to increased skin permeability (Kezic and Nielsen 2009). Data on percutaneous penetration of nanoparticles are scarce. Recently, Labouta et al. 2011a, b showed that in contrast to intact skin barrier, the skin compromised by toluene allows for penetration of nanoparticles of $15 \mathrm{~nm}$ into viable skin (Labouta et al.2011b).

\section{Introduction to the placental barrier}

The human placenta is a unique organ, structurally complex, highly efficient, and metabolically and biosynthetically active (Aye and Keelan 2013). The placenta is responsible for the (bidirectional) transfer of substances between the maternal and foetal circulations including carbon dioxide, oxygen, water, nutrients, hormones, vitamins, and also xenobiotics including drugs and toxic compounds (Desforges and Sibley 2010). In early pregnancy, the human placenta is primarily composed of cytotrophoblasts, which continually fuse to form multinucleate syncytiotrophoblasts as pregnancy progresses. The syncytiotrophoblast consists of two polarized plasma membranes: a maternal-facing microvillous plasma membrane (MVM) and a basal plasma membrane (BM) oriented towards the foetal circulation (Kulvietis et al. 2011, Lager and Powell 2012) (Fig. 2).

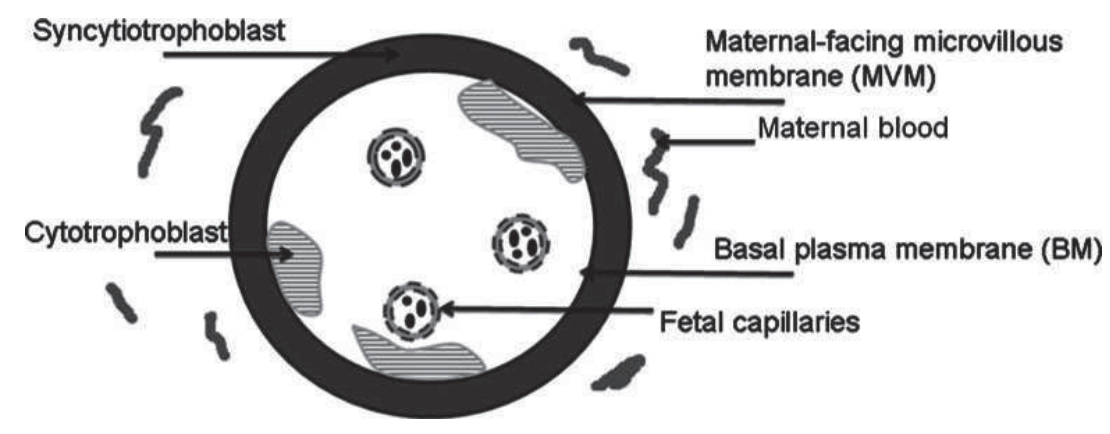

Figure 2. Schematic illustration of the placental barrier as a cross section of a human placental villus. The placental barrier consists of two layers: the syncytiotrophoblast and cytotrophoblast, the latter forming a discontinuous layer. The basal plasma membrane (BM) of the syncytiotrophoblast is oriented towards the foetal circulation, while the maternal-facing microvillous plasma membrane (MVM) faces the maternal blood compartment

The rate-limiting barrier in the human placenta for the permeation of substances between maternal blood and foetal capillaries is the syncytiotrophoblast (Young et al. 2003). Once in the cytoplasm of the syncytiotrophoblast, molecules destined for the foetus exit from the syncytiotrophoblast via the foetal facing basal plasma membrane (BM) (Desforges and Sibley 
2010). At term, the placental diameter varies between 200 and $220 \mathrm{~mm}$ (Benirschke et al. 2006). The diffusion distance between the maternal and foetal circulations varies between 4 and $5 \mu \mathrm{m}$, while in the first trimester the distance varies between 50 and $100 \mu \mathrm{m}$ (Aye and Keelan 2013, Benirschke et al. 2006). Passage across the placenta can occur via simple diffusion, pinocytosis, receptor-mediated uptake, and both active and facilitated transport (Aye and Keelan 2013). The syncytiotrophoblast plasma membranes express numerous transporters which may be regulated by foetal, maternal and placental signals (Lager and Powell 2012). The anatomy and physiology of the human placenta is different from the rodent placenta. The main difference is that in humans the syncytiotrophoblasts arise from fusion of cytotrophoblast cells and form a syncytium with no lateral cell membranes. In rodents, three trophoblast layers are present between maternal blood and foetal blood capillaries.

\section{Overview of currently used in vitro models to study translocation of nanoparti- cles}

Many different in vitro models have been developed to study the translocation of nanoparticles. Most in vitro barrier models culture cells on Transwell inserts, which consist of a permeable membrane separating an apical and a basolateral compartment. Cells are seeded and cultured on the inserts to form a barrier (upon confluence of the cells) between the two compartments. Depending on the cell type selected, the Transwell model can be used to study lung, gastrointestinal, or placental transfer.

Transwells can be used to quantify both uptake of nanoparticles into the cells from the apical compartment and efflux from the cells to the basolateral compartment as a measure of translocation (Fig. 3). Fluorescent polystyrene nanoparticles are the most commonly employed because of their easy detection.

Skin in vitro models are not based on Transwell inserts, because these cell culturing models

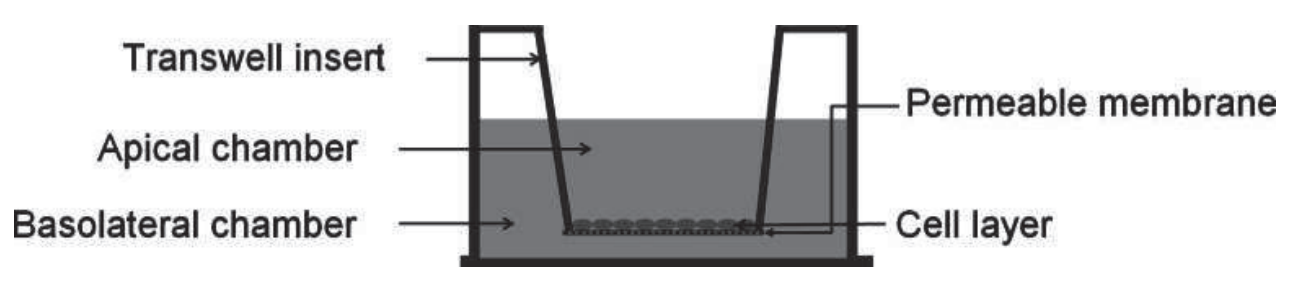

Figure 3. Two-compartment cell culture system contains a permeable cell culture insert, separating two compartments in a Transwell. Cells are seeded and cultured on the inserts to form a barrier between the two compartments 
lack the principal barrier, the stratum corneum. Therefore, ex vivo skin models are mostly used for the in vitro assessment of nanoparticles translocation. Also for the other barriers, especially for the placental barrier and to a lesser extent for the lung and intestinal barrier, ex vivo models are available to test the translocation of nanoparticles.

Table 1 presents an overview of in vitro barrier models currently used to study the translocation of nanoparticles after inhalation, oral intake, dermal exposure, and across the placenta.

\section{Lung in vitro models}

The most frequently used lung epithelial cells lines are A549, Calu-3, H441, and 16HBE140-. Of these cell lines, Calu-3, H441, and 16HBE14o- form tight junctions, but A549 do not (Lehmann et al. 2011, Chowdhury et al. 2010, Geys et al. 2006, Hermanns et al. 2004, George et al. 2015). In in vitro translocation studies, primary rat alveolar cells have also been frequently used (Fazlollahi et al. 2011, Geys et al. 2009, Yacobi et al. 2008).

Besides differences in cell types, in vitro lung barrier models differ in whether they are submerged or cultured at the air-liquid interface. Submerged models have the advantage of being technically simple. However, the culture medium can alter the properties of the nanoparticles, and subsequently their uptake and effects. Air-liquid models mimic more realistically the inhalation exposure; therefore, many air-liquid models have been developed recently (Blank et al. 2006, Brandenberger et al. 2010, Frohlich et al. 2013, Herzog et al. 2013, Holder and Marr 2013, Lenz et al. 2009, 2013, Raemy et al. 2011, Rothen-Rutishauser et al. 2009, Savi et al. 2008, Xie et al. 2012). Disadvantages are the complexity of the system to maintain constant temperature and humidification, and the high costs in comparison with submerged models.

In recent years, co-culture models containing more than one cell type are used to mimic the lung barrier more closely compared with monocultures (Klein et al. 2011). Most models use lung epithelial cells as a basis, those being either primary cells or immortalized cell lines. To obtain co-culture models, different cell types are added to the basic model. The first type of co-culture models includes, in addition to epithelial cells, endothelial cells to mimic the alveolar-capillary barrier (Bermudez et al. 2002, Chowdhury et al. 2010, Hermanns et al. 2010, Hermanns et al. 2004, Papritz et al. 2010). This type of model can be extended by the addition of alveolar macrophages, mast cells, and/or type II alveolar cells. The second type of models does not include endothelial cells, but adds dendritic cells and macrophages to the epithelial cell layer (Brandenberger et al. 2010, Muller et al. 2010, Rothen-Rutishauser et al. 2008, Rothen-Rutishauser et al. 2005). This type of model can also be completed with type II alveolar cells. The third type of models includes, in addition to the epithelial cells, fibroblasts instead of endothelial cells, which can be extended by adding dendritic cells. 


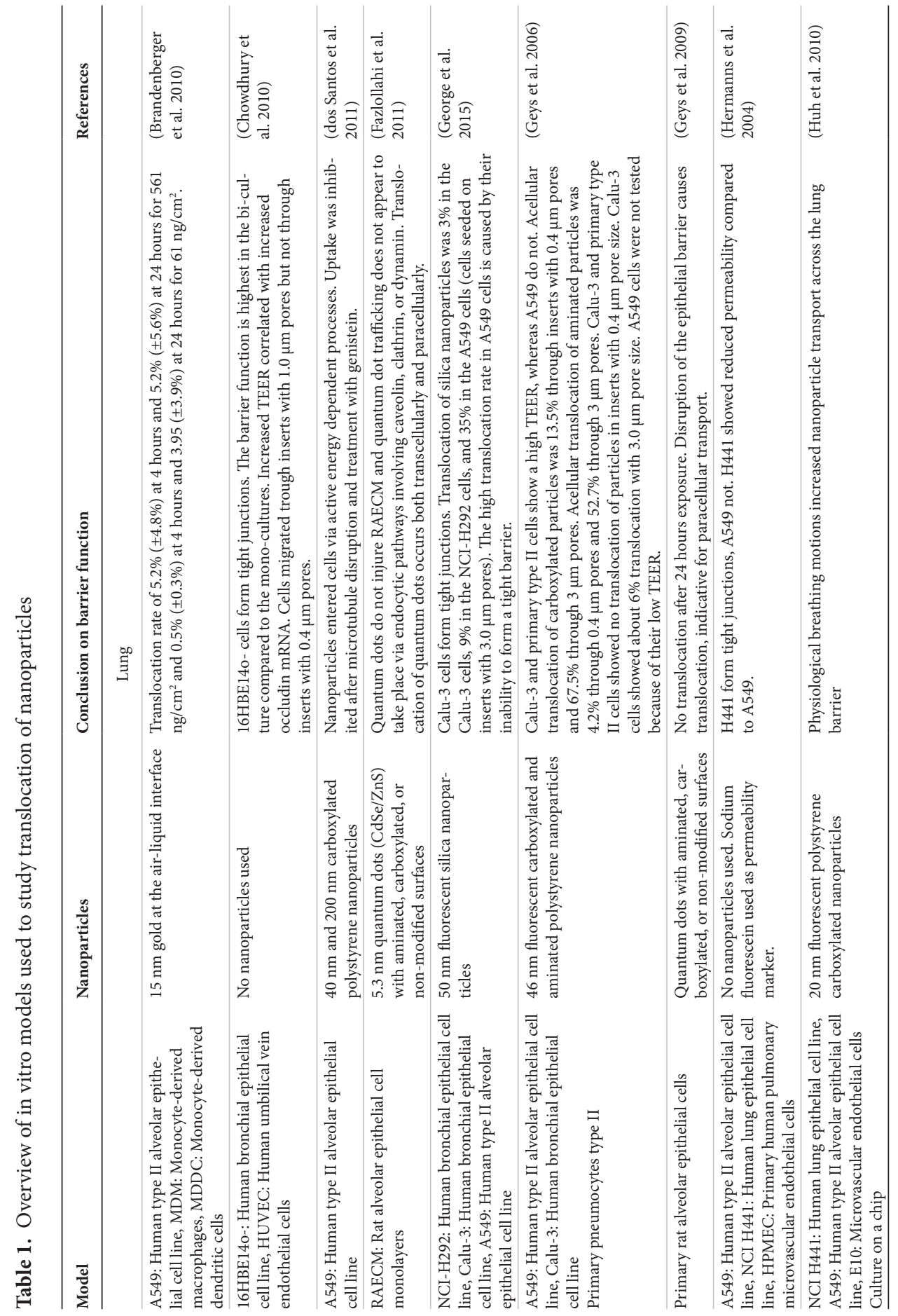




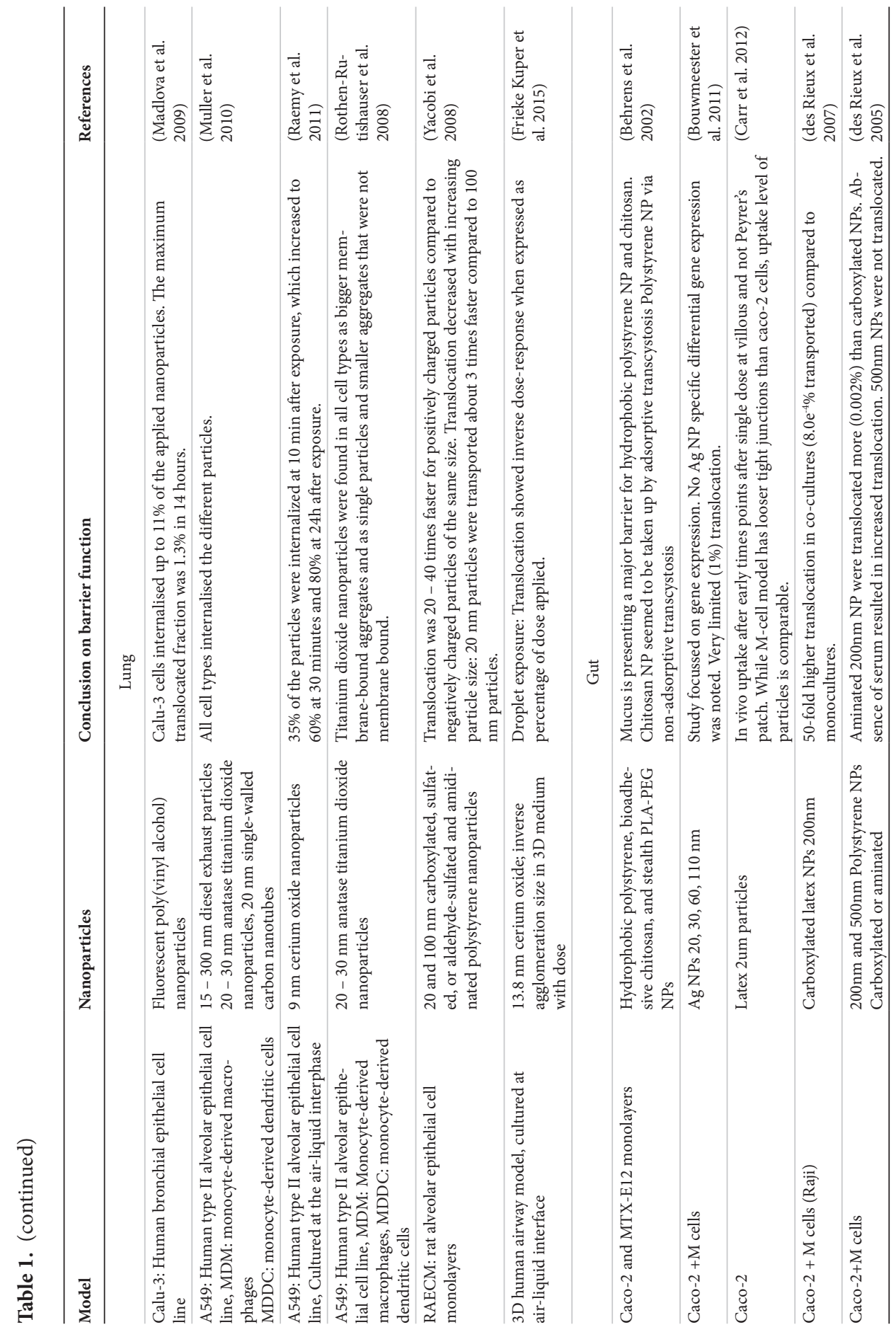




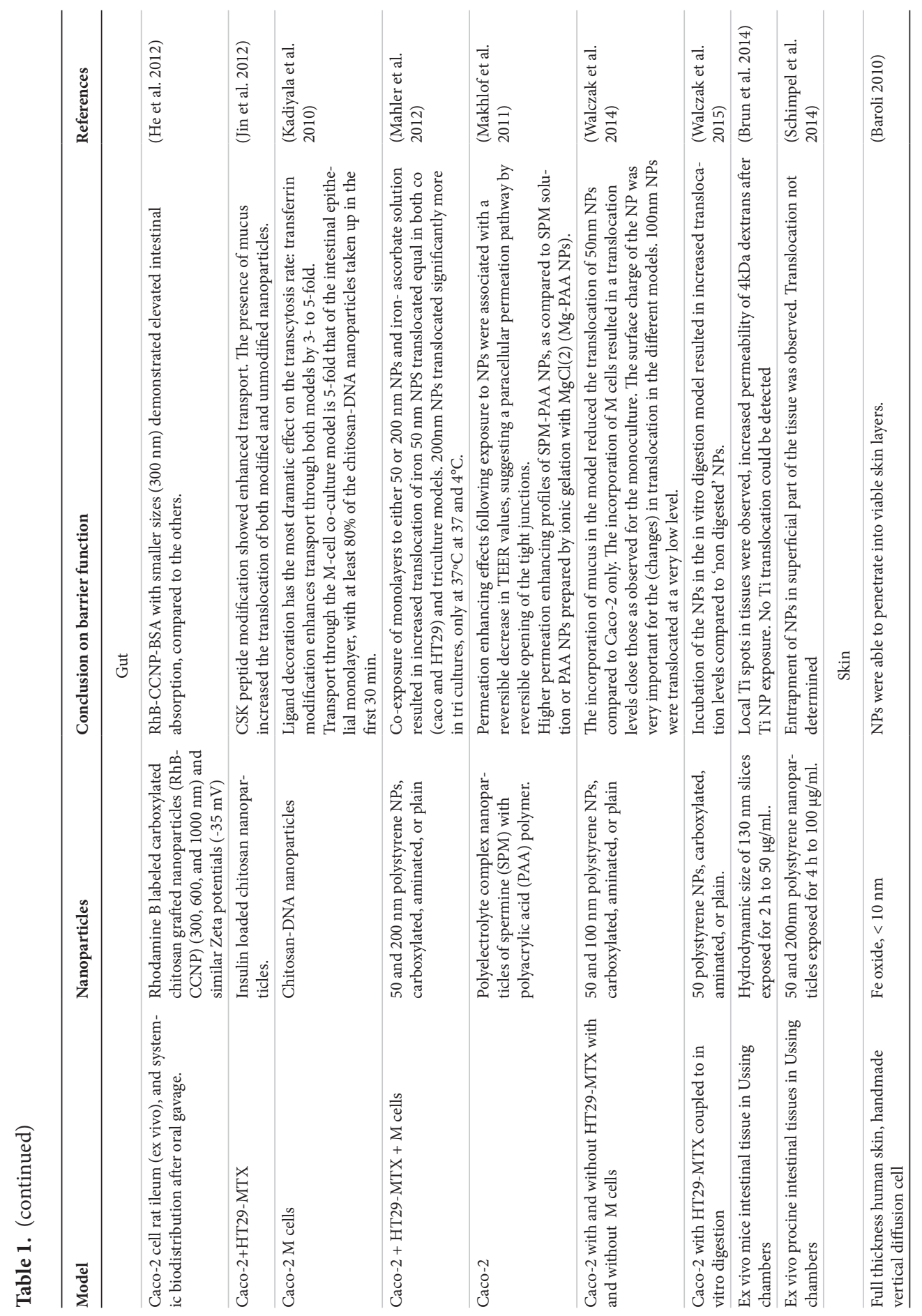




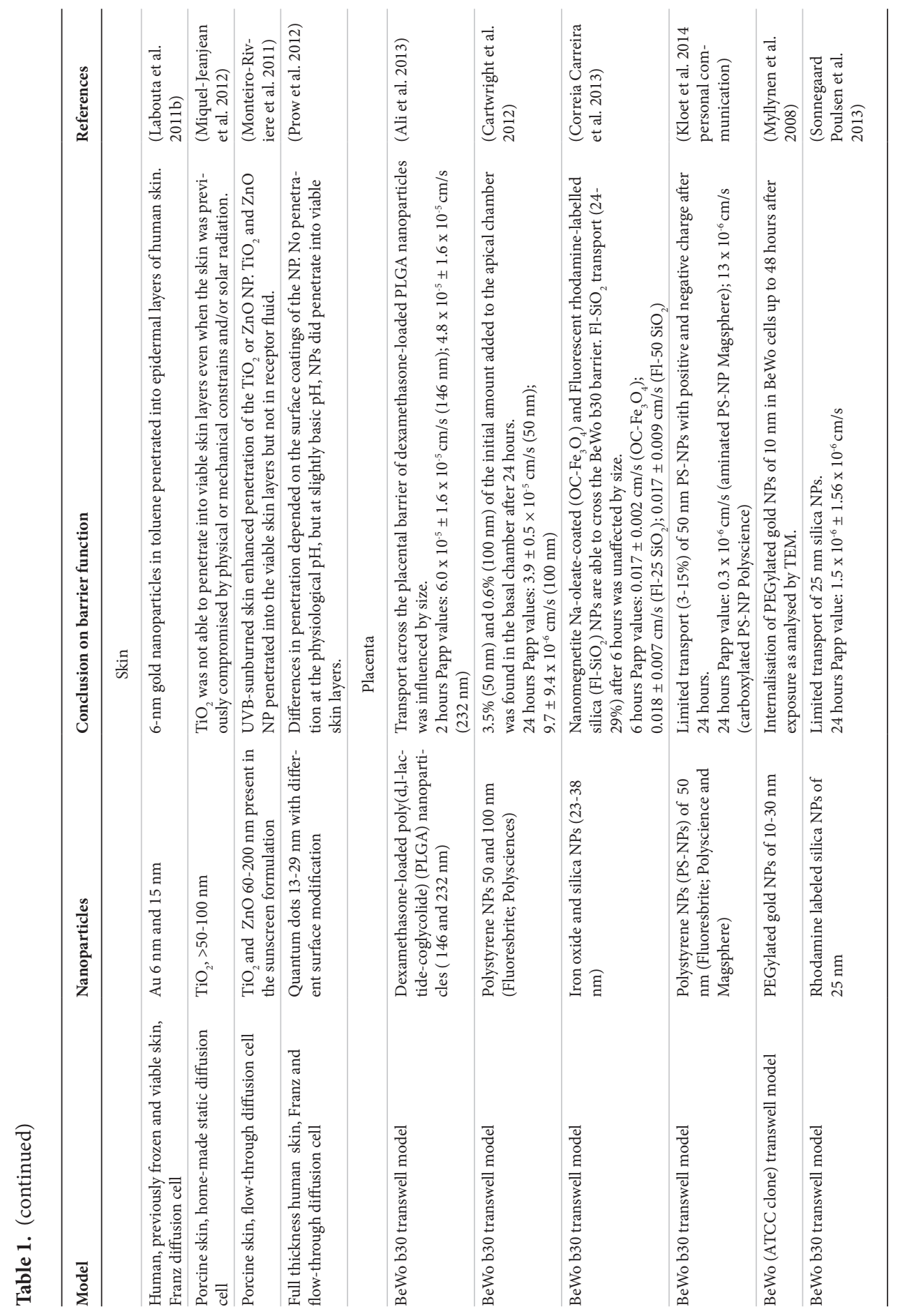




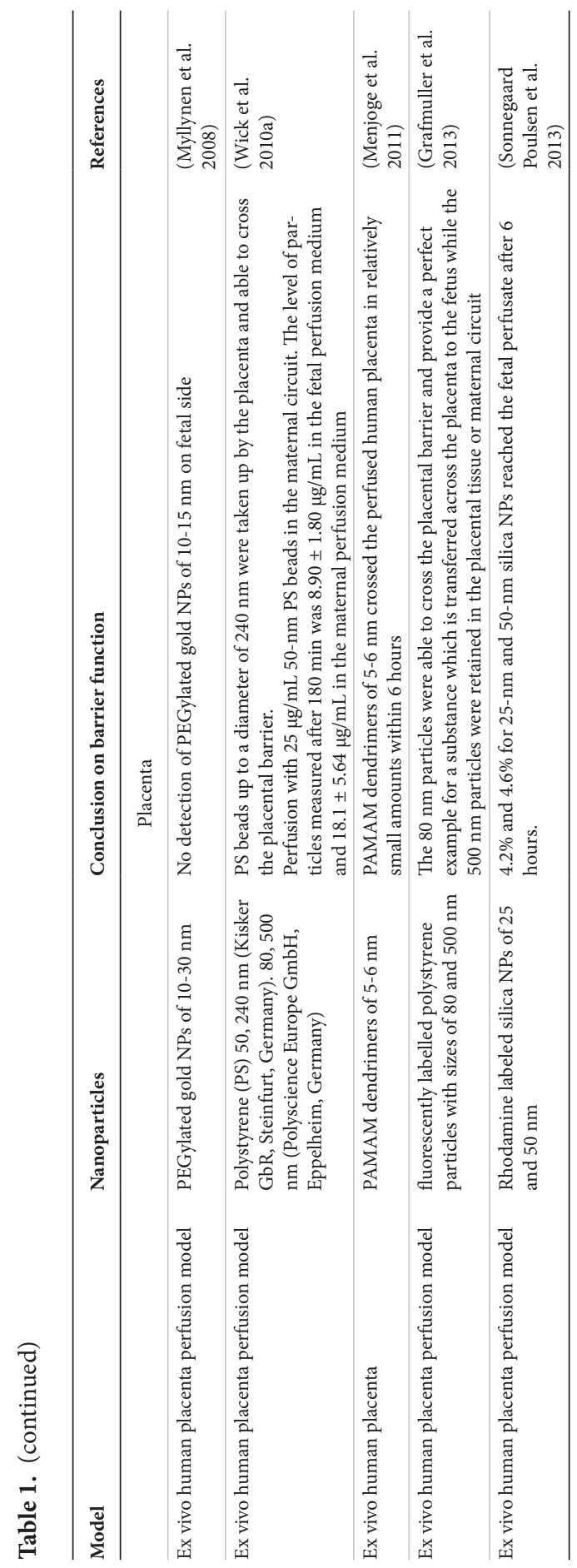


A limited number of nanoparticles have been studied to test translocation across the in vitro lung barrier. These include polystyrene nanoparticles, titanium dioxide nanoparticles, quantum dots, cerium dioxide nanoparticles, gold nanoparticles, silica nanoparticles, diesel particles, and single-walled carbon nanotubes (Table 1). The translocation rate of nanoparticles is higher in models with cells that do not form tight junctions compared with cells that do (Geys et al. 2006, Hermanns et al. 2004, George et al. 2015). Probably, the higher translocation rate is a consequence of paracellular transport, which will not occur in a healthy lung but might occur in a damaged or inflamed lung. The pore size of the permeable membrane of the inserts also influences the translocation rate of nanoparticles: the larger the pore size, the higher the translocation rate (Geys et al. 2006). Therefore, for each single type of nanoparticles, the translocation across the different types of inserts should be tested without cells to assess whether the nanoparticles are not withheld by the insert itself.

Ex vivo tissues as model for the lung are not addressed here. The precision-cut lung slices (PCLS) taken from human and rodent lungs have been used to study translocation and toxicity of nanomaterials. The use of PCLS for translocation of nanoparticle-mediated drug delivery has recently been reviewed by Paranjpe and Muller-Goymann (Paranjpe and Muller-Goymann 2014).

\section{Gut in vitro models}

Orally ingested nanoparticles are exposed to continuously changing conditions while transiting through the gastrointestinal tract, which influences their nature and characteristics (Bellmann et al. 2015). In vitro GI models aim to mimic the gastrointestinal environment as closely as possible, to generate physiologically relevant results. These models focus either on the aspects of dynamically changing GI conditions during digestion by simulation of the transit of nanoparticles along the GI tract from the mouth towards the large intestine (digestion models), or on mimicking translocation and uptake behaviour (in vitro human intestinal epithelium models) (Lefebvre et al. 2014).

Human digestion models can first be simple and static, and mimic only gastric or small intestinal conditions, in which materials are incubated with simulated gastric fluids, simulated small intestinal fluids or buffers at static pH values (Mwilu et al. 2013, Minekus et al. 2014). More complex static models often include most of the relevant GI conditions, i.e. the oral, gastric, small intestinal (and large intestinal conditions) (Oomen et al. 2003, Van de Wiele et al. 2007, Versantvoort et al. 2005). Recently, these models have been used to assess the fate of 60$\mathrm{nm}$ silver nanoparticles, and nanometre-sized silica (synthetic amorphous silica) nanoparticles during digestion (Peters et al. 2012, Walczak et al. 2013). In contrast to static models, more complex dynamic models simulate successive changes in conditions (i.e. $\mathrm{pH}$, secretion of digestive fluids) and transit times (Helbig et al. 2013, Kong and Singh 2010, Minekus et 
al. 1995, Wickham et al. 2009, Zangenberg et al. 2001). Such a dynamic computer controlled model was used to study the behaviour of engineered nanoclay materials (Newsome 2014).

Translocation and uptake of nanoparticles can be addressed by cellular models that can also be divided in relatively simple models or more complex ones (Lefebvre et al. 2014). Amongst the variety of cell models that are available in vitro, intestinal Caco-2 cells (human epithelial colorectal adenocarcinoma cells) are the most commonly used cell type (Miret et al. 2004) in nanoparticle translocation studies. Caco-2 cells are regarded as model cells for enterocytes, the most abundant epithelial cell type in the intestine. Several examples can be found in the literature where monocultures of Caco-2 cells have been used to study the in vitro translocation of nanoparticles, (mainly polystyrene, but also silicon, silver, and organic nanoparticles) (Bhattacharjee et al. 2013, des Rieux et al. 2007, des Rieux et al. 2005, Mahler et al. 2009, Natoli et al. 2012, Nkabinde et al. 2012, Walczak et al. 2014).

A potential drawback of Caco-2 monolayers is the lack of a mucus layer (at least in conventional models), which can, however, be introduced by co-culturing Caco- 2 cells with HT29-MTX cells (human colon adenocarcinoma mucus-secreting cells) (Behrens et al. 2002, Mahler et al. 2009, Scaldaferri et al. 2012, Walczak et al. 2014). The mucus layer consists of mucin glycoproteins that form viscoelastic gels, and it is thinner in the small intestine compared with the large intestine. Though this makes the small intestine a prominent place for nanoparticle uptake, the mucus layer also represents a hindrance allowing selective passage of materials. The mucus can entrap nanoparticles (and thus reduce their translocation) because it poses a physical barrier due to its thickness, density, negative charge, and constant renewal (Cone 2009, Crater and Carrier 2010, Szentkuti and Lorenz 1995). In addition, mucus has a protective function for bio-relevant fluids present in the lumen of the gut, and direct exposure of Caco-2 cells (without a mucus layer) to lumen content stimulants reduces the barrier function of Caco-2 cells models (Ingels et al. 2002). This is probably due to the lack of mucus layer making the Caco-2 cells much more sensitive to direct exposure to the low $\mathrm{pH}$ and high osmolality of the buffers (Westerhout et al. 2014).

To further increase the complex anatomy of the human gut epithelium, human intestine microfold (M) cells are introduced in epithelial monolayers. While M cells in total compose less than 1 percent of the small intestine epithelial cell layer, they are responsible for the uptake and translocation of relatively larger particles (Antunes et al. 2013, Bouwmeester et al. 2011, des Rieux et al. 2007, des Rieux et al. 2005, Kerneis et al. 1997, Martinez-Argudo et al.2007, Walczak et al. 2014). Recently, in vitro digestion models have been linked to in vitro gut epithelial models to study first the digestion of nanoparticles and next the bioavailability of the digested particles in the intestines (Walczak et al. 2015) (see Table 1). 
Several ex vivo gut models have been developed to study the translocation of chemicals. Different approaches are used ranging from in situ perfusion (intestinal loop) models to models in which part of the gut epithelium is excised from animals and maintained in, for example, Ussing chambers for a limited period of time. These models have recently been reviewed by Lefebvre et al. (2014). These authors summarize studies in which the translocation of mainly organic nanoparticles have been evaluated, only a limited number of studies used polystyrene or titanium nanoparticles that have also been used in vitro (see Table 1).

\section{Skin in vitro models}

For the determination of dermal absorption of chemicals, several guidelines have been established by prescribing the type of skin membrane, species, and experimental protocol (OECD 2004). However, specifically for nanoparticles, there are no such guidelines and critical evaluation of the current models is missing. Skin in vitro models differ in the type of the skin membrane (full thickness skin vs. dermatomed skin), species (human vs. animal skin), vehicle and type of the diffusion cell, which hampers comparison and interpretation of the results.

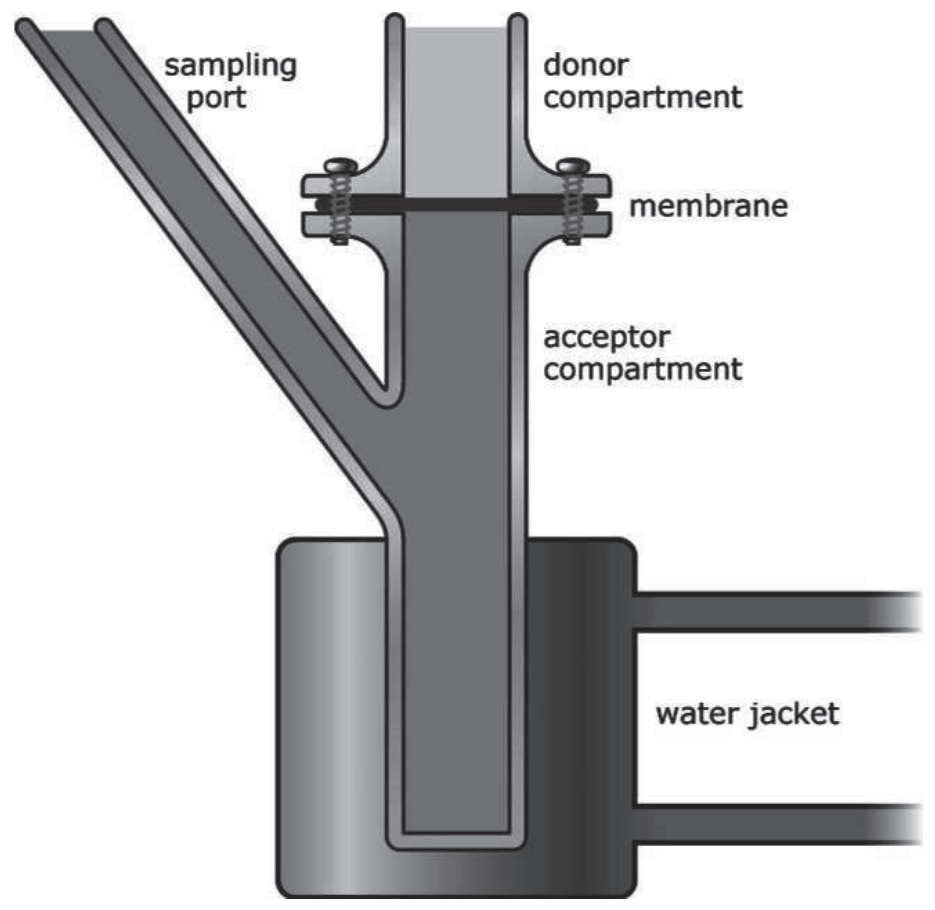

Figure 4. In vitro diffusion chamber to test bioavailability of nanoparticles across the skin barrier. 
In contrast to the lung, intestinal and placental barrier, no Transwells are used for percutaneous penetration studies. The reported in vitro studies on percutaneous penetration of nanoparticles have been performed by using either a Franz static cell or a flow-through diffusion cells. In both systems, the skin membrane is clamped between two chambers, one of which contains a vehicle supplemented with the investigated chemical (donor chamber) and the other one a receptor fluid from which the penetrated chemical will be sampled (Fig. 4) (Jakasa and Kezic 2008). In several studies, human reconstructed skin models have been applied although there were concerns regarding a less well-developed barrier in these models and the absence of the follicular penetration route that might play an important role for translocation of nanoparticles (Labouta et al. 2011a, b). In in vitro assays, usually a cryopreserved skin is used, which might, however, lead to changes of the skin barrier and shrinkage of the hair follicles (Labouta et al. 2011a).

Although human skin is regarded as a gold standard, a large number of studies on percutaneous penetration of nanoparticles use different animal models including mice, rat, and pig skin (Labouta and Schneider 2013). However, structural and morphological differences between human and animal skin, especially concerning the density of the hair follicles, thickness of the skin layers, skin lipid composition, and structure, could certainly affect the NP percutaneous penetration (Labouta and Schneider 2013). Another problem encountered by using hairy animals is damage of the skin barrier due to hair clipping.

\section{Placenta in vitro models}

Models for studying transplacental transport have long been based on the perfused isolated human placenta studied in ex vivo study designs. The perfused isolated human placenta ex vivo model presents a directly relevant alternative that maintains the complexity of the intact placenta (Saunders 2009). Transport studies in the ex vivo intact placenta are technically challenging and require large quantities of substances to be tested. Therefore, models using representative placental cell lines in Transwell study designs are being developed as in vitro alternatives. Most commonly used human placental cell lines are the BeWo, Jar, and JEG-3 cell lines, which have been applied to study transplacental transfer of a variety of drugs and compounds. The most popular cellular model consists of the BeWo cell line, representing a choriocarcinoma-derived placental cell line that strongly resembles cytotrophoblastic cells. The BeWo b30 subclone can be grown on Transwell inserts to form confluent cell layers, enabling the quantification of both uptake into the cells from the apical compartment (maternal side) and efflux from the cells to the basolateral compartment (foetal side) (Buerki-Thurnherr et al. 2012). In some studies these models have been validated by comparison of the transport rate across the Transwell cellular BeWo b30 layer and the transport rates detected in ex vivo placental models for the same compounds (Li et al. 2013, Poulsen et al. 2009). 
Although the type and nature of the nanoparticles studied in in vitro placental models are different and the number of studies is limited, some general observations can be made. Nanoparticles can be transported across the placental barrier, where their size and type of nanoparticles influence the efficiency of the transport. Furthermore, in contrast to drugs and other xenobiotic bulk chemicals, transport of several nanoparticles across the placental barrier appears to be highly variable. The latter conclusion can be derived from the observation that for the nanoparticles studied so far the amount (\%) of the nanoparticles transported across the BeWo b30 cell layer varies from 0.6 to $29 \%$ in $6 \mathrm{~h}$ (Table 1). Comparison of this transport rate to that reported in the BeWo model for several chemicals mounting up to $30 \%$ in $2 \mathrm{~h}$ reveals that the transport of nanoparticles across the BeWo cell layer, just as that of chemicals, may be limited or significantly depending on the type of nanoparticles. This implies that a validated in vitro model to quantify the potential transport of nanoparticles across the placental barrier would be of high value to set priorities for further in vivo testing, thereby avoiding in vivo testing of all newly developed nanoparticles.

\section{General remarks on currently used in vitro models to study translocation of nanoparticles}

Currently used in vitro models to study translocation of nanoparticles are mostly based on cell culturing on Transwell inserts or on the use of ex vivo tissues. Transwell inserts can differ in the type of plastic they are made of and in the pore size of the permeable membrane. These differences between the Transwell inserts influence the translocation rate (Cartwright et al. 2012, Geys et al. 2006). Transwell inserts with a larger pore size give increased translocation rates compared with inserts with smaller pore sizes. In addition, some types of plastic of the Transwell inserts yield decreased translocation due to nanoparticle adsorption on the material surface. Therefore, for each single type of nanoparticles, the translocation across the different types of inserts should be tested without cells to assess whether the nanoparticles are not withheld by the insert itself.

Next to this, different cell types are used, which clearly influences the translocation and the mechanism of translocation. When cell types lack the formation of tight junctions, nanoparticles can easily pass between the cells resulting in a higher translocation rate compared with cell types that do form tight junctions. To prevent paracellular transport, epithelial cells forming tight junctions are preferred. As already discussed, the incorporation of a mucus layer in gut epithelial models decreases the translocation (Walczak et al. 2014). This mucus layer might be a very important barrier for nanoparticles in vivo because of electrostatic repulsion (for negatively charged nanoparticles) and mucus entrapment (for positively charged nanoparticles) (Husain et al. 2001, Lai et al. 2007, Norris et al. 1998, Szentkuti and Lorenz 1995). Similarly, such a mucus layer might also be essential for in vitro lung barrier models, since nanoparticles first come in contact with the pulmonary surfactant if they are deposited 
in the lungs. Lastly, the use of specific cells types in co-culture on top of an epithelial layer can alter the translocation rate of the nanoparticles. For instance, alveolar macrophages in lung models can decrease the translocation rate by uptake of nanoparticles, while $\mathrm{M}$ cells in intestinal models can enhance translocation. The addition of these specific cell types can improve the in vitro models by more closely mimicking the in vivo situation in which, for example, the lungs are protected from particles via phagocytosis by alveolar macrophages.

Some reported in vitro models use primary cells, while others use immortalized cell lines. On one hand, primary cells have a more differentiated phenotype compared with cell lines. On the other hand, the isolation of primary cells is often experimentally challenging: the cells dedifferentiate after isolation, differ from batch to batch, and proliferate to a limited extent. Cell lines are easy to work with, well characterized and more homogenous, but they show only few characteristics of differentiated cells. Overall they only poorly represent the in vivo situation (Hartung et al. 2002, Klein et al. 2011). In in vitro lung translocation studies, primary rat alveolar cells have been frequently used (Fazlollahi et al. 2011, Geys et al. 2009, Yacobi et al. 2008). Recent, biologically complex human intestinal tissues have been cultured using human (induced) pluripotent stem cells, resulting in human intestinal organoids that have secretory and absorptive functions (Brugmann and Wells 2013). Comparable systems have now been modified into mature epithelial, functional and polarized monolayers grown on Transwell membranes, which are suitable for translocation studies (VanDussen et al. 2014).

Ex vivo models maintain the complexity of the physiological barriers (Saunders 2009). However, transport studies in ex vivo models are technically challenging, time consuming, and can require large amounts of the test substance.

Advances in the field of miniaturized and microfluidic devices have recently led to the concept of organ-on-a-chip models (Bhatia and Ingber 2014, Huh et al. 2012, Moraes et al. 2012, van der Meer and van den Berg 2012), which can be seen as hybrid devices combining cells and microfabricated structures aiming to recapitulate the dynamic physical, cellular, and functional features of human tissues (Ardavin et al. 2001, Huh et al. 2010. 2013, Schimek et al. 2013, Wagner et al. 2013). These devices first provide a high control on the cell microenvironment (e.g. physical and chemical parameters) together with dynamic culture conditions, since they are embedded in a microfluidic format (Whitesides 2006). Furthermore, these devices can include active elements, which allow exposing cells to mechanical stimuli and surface strains, by stretching the substrate on which they are grown (Sinha et al. 2015). These approaches mostly rely on 3D cell culture conditions, which are acknowledged to better mimic the in vivo conditions compared with conventional monolayer models (Harink et al. 2013). 
Over the years, examples of organ-on-a-chip models in the literature have diversified and, for instance, include gut (Kim and Ingber 2013), lung (Huh et al. 2007, 2010, Nalayanda et al. 2010), or even blood-brain barriers (Griep et al.2013, Huh et al. 2013, Wolff et al. 2015). These models can be of particular interest to the field of nanotoxicology. Huh et al., for instance, have developed a complex lung-on-a-chip model, which recapitulates movements associated with breathing that is accompanied by cyclic stretching of cells (Huh et al. 2010). This device has been applied for NP testing, showing that breathing motion is essential for such nanotoxicological assays. The same group, using the same principle, later proposed a gut-on-a-chip model integrating peristaltic motion associated with digestion. Interestingly, exposed to a combination of dynamic culture and mechanical strain, Caco- 2 cells were producing mucus and microvilli features were formed (Kim and Ingber 2013). Finally, organon-a-chip devices are amenable to parallelization and automation; they are easily coupled to virtually any detection technique, and different organ models can even be combined on one device (Wagner et al. 2013) to eventually mimic the whole body/organism. The availability of such complex and in vivo-like models is expected to facilitate the implementation of the 3R's legislation regarding animal experimentation (Marx et al. 2012, van de Stolpe and den Toonder 2013).

\section{Accuracy of current in vitro translocation models compared to in vivo data}

In vitro translocation studies show mostly limited transport of nanoparticles across the lungs, gut, skin, and placenta. In the sections below, we discuss the comparison between in vitro data and available in vivo data. We included in vivo studies that measured the bioavailability of similar nanoparticles compared to their in vitro counterparts. However, most in vivo data come from separate studies in which not exactly the same type of nanoparticle is used: for instance, the nanoparticles differ in size. Another marked difference is that the in vitro models are composed of cells (often cell lines) with a human origin, while the in vivo models mostly are rodents. Therefore, we first compared data from studies using nanoparticles of the same chemical composition although they sometimes differed for other characteristics. In Table 2 , we included studies that used exactly the same nanoparticles in both an in vivo and an in vitro design.

\section{Lung barrier}

The translocation rate between in vitro lung barrier models and in vivo inhalation studies differs depending on the tested nanoparticles (Table 2). In addition, some studies determined the cellular uptake and not the translocation rate of nanoparticles. The information on their localization can also be used in the comparison between in vitro and in vivo data. 
For the most frequently used polystyrene nanoparticles, the translocation rate, both in vitro and in vivo, is very low 1 day after exposure. Depending on the pore size of the inserts, the translocation amounts to $0-6 \%$ in vitro against $0.05-2 \%$ in vivo. However, one in vivo study shows accumulation over time of polystyrene nanoparticles in the thymus (Sarlo et al. 2009), which cannot be demonstrated in an in vitro lung model. One in vitro study reported a size dependent translocation rate (Yacobi et al. 2008), which is in line with the size-dependent distribution shown after oropharyngeal aspiration (Sarlo et al. 2009). Also for gold nanoparticles, the in vitro translocation rate ranges from 0.5 to $5.2 \%$ (Brandenberger et al. 2010). For comparison this can be compared to the in vivo translocation to the liver of 0.7 to 2.8 $\%$; clearly, this is an underrepresentation of the complete systemic availability (Sadauskas et al. 2009, Sung et al. 2011, Takenaka et al. 2006, Yu et al. 2007). For cerium dioxide, quantum dots, silica, and titanium dioxide nanoparticles, different translocation rates are found in vitro and in vivo. For cerium dioxide, the uptake rate in vivo is very low, and only about $10 \%$ of the inhaled cerium dioxide was detected in the lungs (Geraets et al. 2012), while $80 \%$ of the particles was internalized in cells in vitro (Raemy et al. 2011). After inhalation exposure, quantum dots could be detected in the liver and kidney at relatively high amounts of 15 and $5 \%$, respectively (Ma-Hock et al. 2012). However, in vitro, no translocation of quantum dots across primary rat alveolar epithelial cells was observed (Fazlollahi et al. 2011, Geys et al. 2009). Silica nanoparticles were able to translocate across monolayers of Calu-3, NCI-H292, and A549 cells in vitro (George et al. 2015), but were not detected in tracheobronchial lymph nodes after inhalation exposure (Arts et al. 2007). However, the silica content was not measured in any other tissue or organ besides the lung and lymph nodes, so there might have been some in vivo translocation to the blood, liver, and other organs. Finally, titanium dioxide nanoparticles were internalized by A549 epithelial cells, monocyte-derived macrophages, and monocyte-derived dendritic cells in vitro (Muller et al. 2010, Rothen-Rutishauser et al. 2008), while the in vivo studies showed that most nanoparticles retained in the alveolar space in the lung-lining fluid directly after exposure, and were found later mostly inside alveolar macrophages (Creutzenberg et al. 2012, Geiser et al. 2005, Oberdorster et al. 1994). 


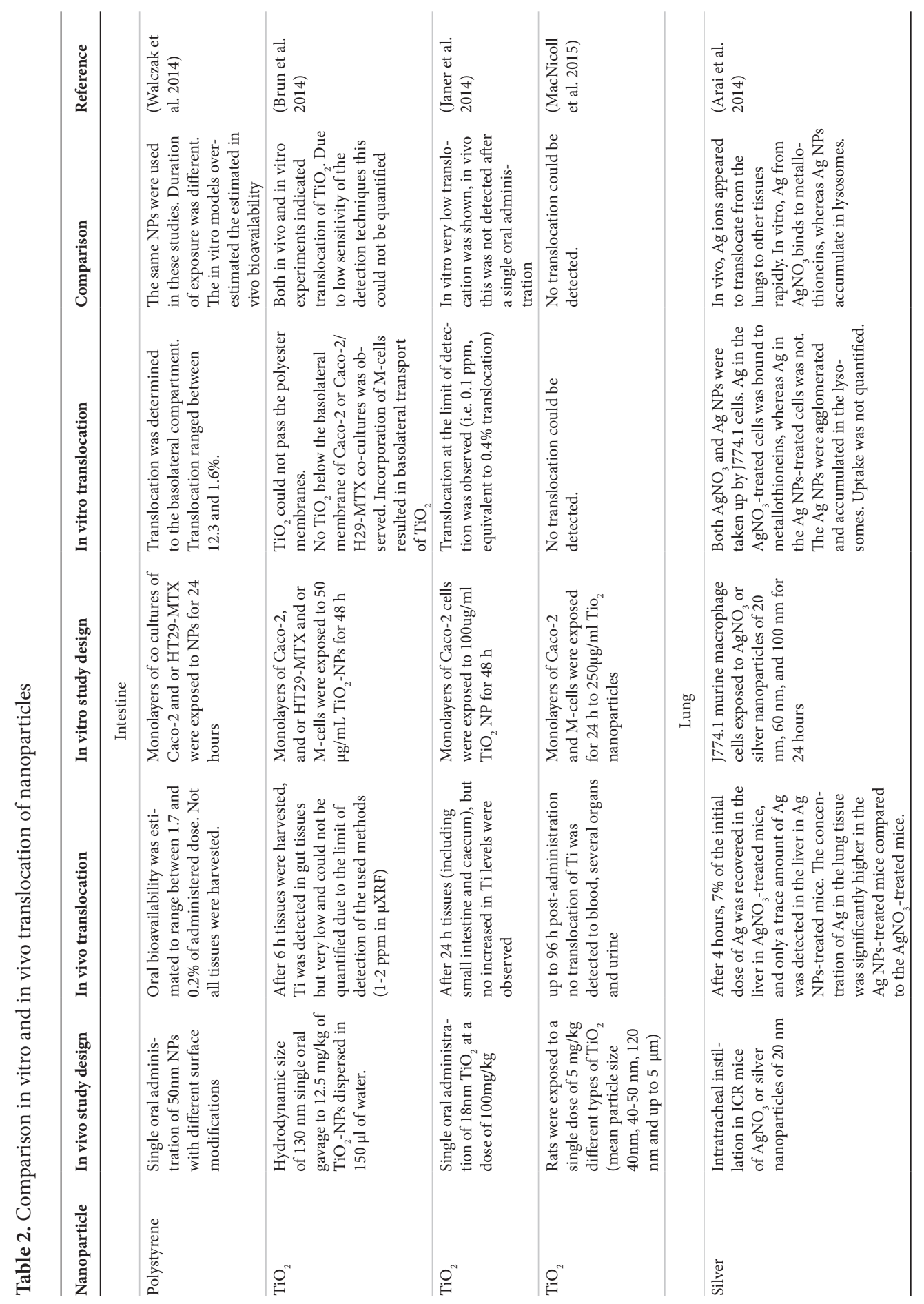




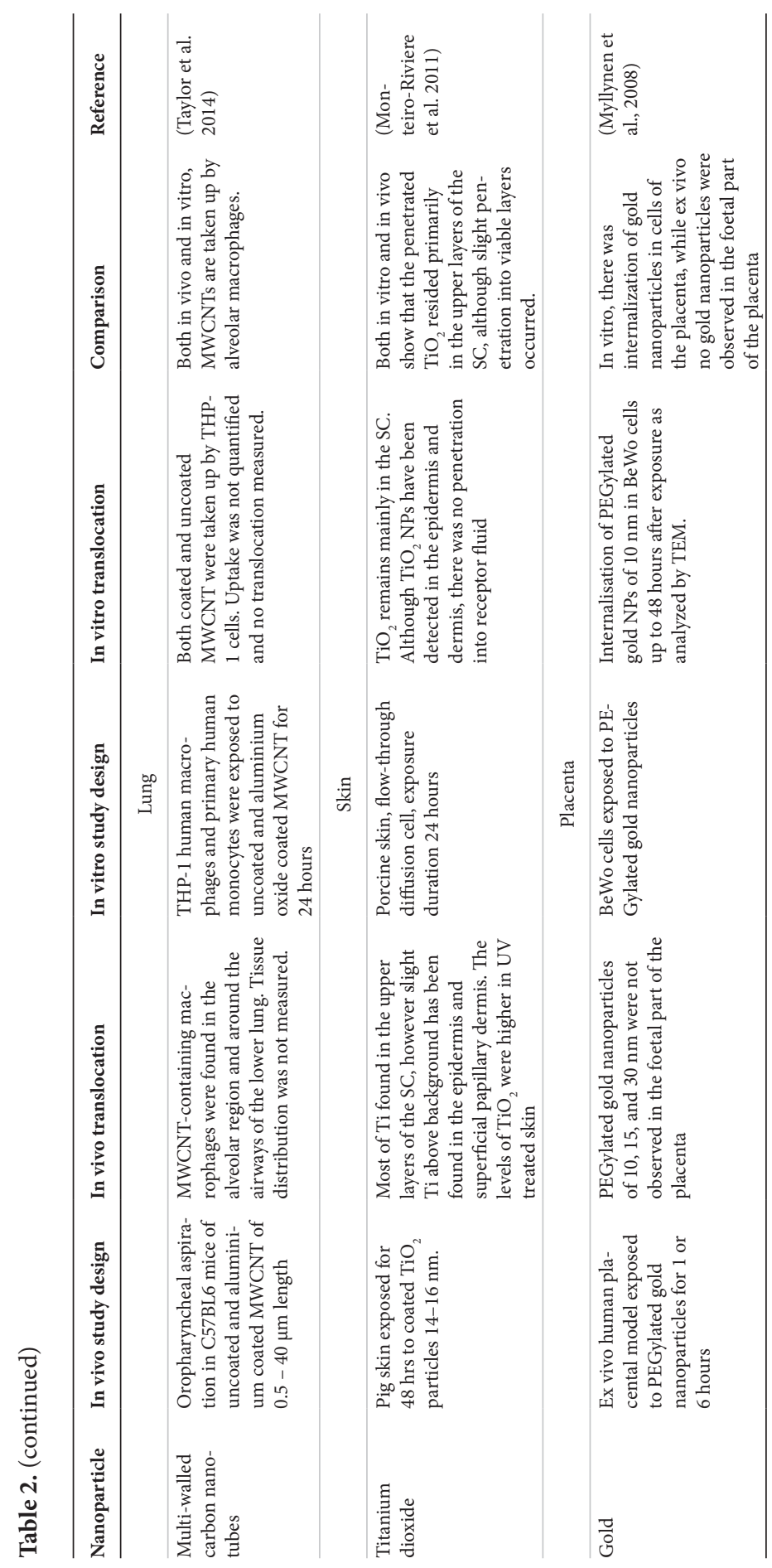


The above-mentioned studies did not test exactly the same nanoparticles for the in vitro and in vivo set-ups. Arai et al. (2014) did test the same silver nanoparticles in vitro and in vivo. However, they did not measure the in vitro NP translocation but the presence of silver material in cells. They concluded that, in vivo, silver ions appear to translocate from the lungs to other tissues rapidly. In vitro, $\mathrm{Ag}$ from $\mathrm{AgNO}_{3}$ binds to metallothioneins, whereas Ag nanoparticles accumulate in lysosomes (Arai et al. 2014). Multi- walled carbon nanotubes (MWCNTs) were jointly tested in vitro and in vivo. However, this study focused on the development of pulmonary fibrosis and did not measure tissue distribution or translocation. The results did show, however, that MWCNTs are taken up by alveolar macrophages both in vivo and in vitro (Taylor et al. 2014).

\section{Intestinal barrier}

Several in vivo oral studies have been performed, but only a very limited number of those in vivo studies can directly be compared to in vitro studies. Here, we focus only on studies that evaluated the uptake of food-relevant nanoparticles such as silica and titanium dioxide. In addition, studies that used model materials such as polystyrene are discussed, as polystyrene nanoparticles have also been used in vitro (Table 1).

Some studies investigated the in vitro and in vivo translocation of titanium dioxide across the gut epithelium. Six hours after a single oral administration of $130-\mathrm{nm} \mathrm{TiO}_{2}$, titanium could be detected in gut tissues, but due to the low concentrations, it could not be quantified (Brun et al. 2014). Janer et al. (2014) did not observe any increase in tissue concentration in vivo 24 $\mathrm{h}$ after a single dose of $100 \mathrm{mg} / \mathrm{kg} \mathrm{TiO}$ of $18 \mathrm{~nm}$, while very low translocation was observed in vitro (Janer et al. 2014). In another study, rats were exposed to $5 \mathrm{mg} / \mathrm{kg}$ of different types of $\mathrm{TiO}_{2}$ nanoparticles (mean particle size $40 \mathrm{~nm}, 40-50 \mathrm{~nm}, 120 \mathrm{~nm}$ and up to $5 \mu \mathrm{m}$ ), and up to $96 \mathrm{~h}$ post-administration, no translocation of titanium was detected to blood, several organs and urine. Also, no translocation was observed in vitro (MacNicoll et al. 2015). In rats exposed for 30 days to $200 \mathrm{mg} / \mathrm{kg}$ body weight (bw) 75- $\mathrm{nm} \mathrm{TiO}_{2}$ nanoparticles, no increased titanium levels were detected in blood, liver kidney and spleen, while effects on liver and other organs have been reported (Wang et al. 2013). Titanium nanoparticles were also orally administered to rats, with a dose of 1 or $2 \mathrm{mg} / \mathrm{kg} \mathrm{TiO}$ nanoparticles with a primary size of 20-60 nm (large agglomerates were present) for 5 days (Tassinari et al. 2014). Only a limited number of tissues were collected; interestingly, in spleen elevated Ti concentrations were found in the high dose group. Detailed analysis revealed the presence of 130-nm (sp ICPMS) or 200-400 nm (SEM-EDX) materials in spleens (Tassinari et al. 2014).

Like titanium dioxide, silica is commonly used as food additives. Therefore, the oral uptake of silica nanoparticles was studied after 28 and 90 days of exposure to food-grade synthetic amorphous silica. Limited uptake was observed: only after 90-day exposure to 2500 
$\mathrm{mg} / \mathrm{kg}$ bw elevated Si levels were found in spleen (van der Zande et al. 2014). More studies are available that focussed on model polystyrene nanoparticles, and they generally highlight the dependence of uptake and accumulation of polystyrene nanoparticles on several factors, including their size, surface charge, and type of coating material (Araujo et al. 2014, Hillery et al. 1994, Hillyer and Albrecht 2001, Hussain and Florence 1998, Hussain et al. 1997, Jani et al. 1989). In general, smaller polystyrene nanoparticles were taken up across the GI tract to a higher extent than the larger ones (Jani et al. 1990); the non-ionic more than the carboxylated ones (Jani et al. 1989) and 407 poloxamer-coated more than 188 poloxamer-coated nanoparticles (Hillery and Florence 1996, Hussain et al. 1997).

The estimated oral bioavailability of 50-nm polystyrene nanoparticles varied between studies from 0.2 to $1.7 \%$ (Walczak et al., submitted) to $6.6 \%$ (Jani et al. 1990). Strikingly, much higher bioavailability (23\%) was reported for 500-nm polystyrene nanoparticles (Hussain et al. 1997), while their 1- $\mu \mathrm{m}$-size counterpart's nanoparticles had a lower uptake $\left(2 \times 10^{-6} \%\right.$ detected in lymph fluid) (Seifert et al. 1996). Also, the amounts of polystyrene nanoparticles associated with intestinal tissues that were reported by Walczak (between 0.38 and $0.74 \%$ depending on the type of polystyrene nanoparticles, calculated as a sum of the small- and large intestinal walls), were lower than the ones reported by others for 60-nm nanoparticles (between 1.5 and $10 \%$, depending on the type (i.e. surface chemistry) of polystyrene nanoparticles used) (Hillery and Florence 1996, Hillery et al. 1994). Comparison of the oral in vivo bioavailability ( 0.2 and $1.7 \%$ ) (Walczak 2015), with the in vitro translocation values (1.6 to $12.3 \%$ ) of the same 50-nm polystyrene nanoparticles (Walczak et al. 2014), shows lower uptake values in the in vivo model. Therefore, the in vitro model used by Walczak et al. appears to overestimate the in vivo translocation.

\section{Skin barrier}

In the literature, various in vitro assays (based on ex vivo skin tissue) have been used to determine percutaneous penetration of nanoparticles. However, the scarcity of in vivo human data hampers proper evaluation of these models. The vast majority of in vitro studies focused on $\mathrm{TiO}_{2}$ and $\mathrm{ZnO}$ nanoparticles found in sunscreens. Most, although not all in vitro studies find that these nanoparticles do not penetrate beyond the superficial layers of the stratum corneum (Cross et al. 2007, Mavon et al. 2007, Wu et al. 2009, Zvyagin et al. 2008). One of the disadvantages of in vitro assays is that exposure duration is limited to $24 \mathrm{~h}$, although long-term exposures would be closer to the real-life situation. Wu et al. (2009) compared in vitro and in vivo penetration of nanoscale $\mathrm{TiO}_{2}(4 \mathrm{~nm}$ and $60 \mathrm{~nm})$ in two animal models (hairless mice and pigs). After in vitro dermal exposure, $\mathrm{TiO}_{2}$ nanoparticles were not detected beyond the stratum corneum. However, in vivo, 30-day dermal exposure to the same nanoparticles in hairless mice revealed, that in contrast to short-term in vitro exposure, $\mathrm{TiO}_{2}$ 
nanoparticles do reach viable skin layers (Wu et al. 2009). Furthermore, after 60-day dermal exposure, $\mathrm{TiO}_{2}$ nanoparticles could penetrate through the skin, reach different tissues and induce diverse pathological lesions in several major organs (Wu et al. 2009). Deeper, although minimal, penetration into epidermal layers has also been found for $\mathrm{ZnO}$ nanoparticles in an in vivo study in human volunteers (Leite-Silva et al. 2013). In a parallel in vitro-in vivo study, Monteiro- Riviere et al. (2011) investigated the penetration of $\mathrm{TiO}_{2}$ and $\mathrm{ZnO}$ nanoparticles in UVB-damaged porcine skin. Under both conditions, $\mathrm{TiO}_{2}$ and $\mathrm{ZnO}$ NP predominantly resided in the stratum corneum, although small amounts of $\mathrm{TiO}_{2}$ and $\mathrm{ZnO}$ were also detected in the viable skin layers. On the other side, not all in vivo studies report penetration of nanoparticles beyond the stratum corneum (Monteiro- Riviere et al. 2011). For instance, Zvyagin et al. found in an in vivo study with human skin no penetration of $\mathrm{ZnO}(26-30 \mathrm{~nm})$ into the viable layers (Zvyagin et al. 2008). This is consistent with the findings reported by Mavon et al. showing no penetration of $\mathrm{TiO}_{2}$ into the viable epidermal layers of human skin either in vivo or in vitro (Mavon et al.2007). Obviously, the discrepancy in the results cannot be explained solely by the differences between in vitro and in vivo data but also by the characteristics of the used nanoparticles (size, coating), exposure duration and sensitivity of the detection methods.

Another frequently investigated NP is silver, which is used on a large scale in medicinal and consumer products. Larese et al. found in an in vitro study with human skin that smaller silver nanoparticles $(30 \mathrm{~nm}$ ) can penetrate across the stratum corneum into the upper layers of the epidermis (Larese et al. 2009). This is consistent with in vivo data obtained in human volunteers (George et al. 2014) showing that silver nanoparticles could penetrate as deep as the reticular dermis. Zhu et al. showed, using a highly sensitive detection method, that the penetration depth of Ag nanoparticles could exceed the stratum corneum thickness (Zhu et al. 2015).

\section{Placental barrier}

As described above, the transport efficiency of nanoparticles across the human placenta is likely to be different from that in rodents (Wick et al. 2010). To study the translocation over the human placental barrier, dual recirculation human placental (ex vivo) perfusion models are used. In such a design the translocation of 50, 80-, 240-, or 500-nm polystyrene was studied, under highly controlled conditions (i.e. translocation of a marker compound) (Wick et al.2010). After a single administration, 50-, 80-, and 240-nm polystyrene nanoparticles were observed in the foetal circulation (foetal to maternal ratios $0.4 ; 0.4 ; 0.1$, respectively), while the 500-nm polystyrene nanoparticles were retained in the placenta (Wick et al. 2010). In vitro similar results were found, where the translocation rate of 50-nm polystyrene nanoparticles was larger compared with 100-nm nanoparticles (Cartwright et al., 2012). Myllynen et al. perfused ex vivo human placentas with 10-, 15-, and 30-nm PEGylated gold nanoparticles 
(for up to $6 \mathrm{~h}$ ) and did not observe any PEGylated gold nanoparticles in the foetal part of the placenta (Myllynen et al. 2008). In vitro, they observed internalization of PEGylated gold nanoparticles of $10 \mathrm{~nm}$ in BeWo cells up to $48 \mathrm{~h}$ after exposure (Myllynen et al. 2008).

In rodents, fluorescent polystyrene nanoparticles were administered via the extraembryonic tissue. The embryos were ex vivo incubated for $12 \mathrm{~h}$ with 20-, 100-, and 500- nm carboxylated and 200-nm amino-conjugated/terminated polystyrene nanoparticles. The 20 -nm carboxylated nanoparticles were distributed in the embryonic and extraembryonic germ layers of ectoderm, mesoderm, and endoderm. The 100 and 500-nm carboxylated polystyrene nanoparticles accumulated in extraembryonic tissue. Interestingly, the 200-nm amino-conjugated particles can pass into the embryos (Tian et al. 2009). For 5-nm gold nanoparticles, $0.018 \%$ of the administered dose (118 $\mu \mathrm{g} / \mathrm{kg}$ bw intravenous) administered to rats at day 19 of gestation was detectable in the embryo (Takahashi and Matsuoka 1981).

In addition, studies with silica nanoparticles showed that $70 \mathrm{~nm}$, but not 300 or $1000 \mathrm{~nm}$ reached the brain and liver of the foetus after i.v. administration to the mother (Yamashita et al. 2011), while there was limited transport of $25-\mathrm{nm}$ silica nanoparticles in vitro (Sonnegaard Poulsen et al. 2013). In the same study of Yamashita et al. 63-nm $\mathrm{TiO}_{2}$ nanoparticles were detected by TEM in brains and livers of foetuses (Yamashita et al. 2011). In another study, surface modified 28-30-nm iron oxide nanoparticles were administered (intraperitoneal) to mice from gestation days 9-16. Nanoparticles with a positive zeta potential in water [coated with hydrophilic polyethyleneimine (PEI)] were detected in the livers of foetuses 1 day after dosing of the dams, while negatively charged nanoparticles (coated with acrylic acid) could not be found in the foetuses (Di Bona et al. 2014). In vitro, iron oxide nanoparticles were able to cross a BeWo cell layer (Correia Carreira et al. 2013).

Based on these studies, it can be concluded that nanoparticles can pass the placenta of rodents and humans (ex vivo). This translocation is size- and surface charge- dependent. Although there are few studies available to compare the in vitro translocation to the in vivo or ex vivo translocation, for polystyrene nanoparticles, size-dependent translocation has been observed both ex vivo and in vitro. However, for gold nanoparticles and iron oxide nanoparticles the in vitro translocation was higher compared with the ex vivo or in vivo translocation, while for silica nanoparticles it was lower. 


\section{ANALYTICAL TECHNIQUES TO QUANTIFY AND CHARACTERIZE NANOPAR- TICLE TRANSLOCATION IN VITRO AND IN VIVO}

Meaningful interpretation and comparison of the results obtained using different in vitro experiments and extrapolation to in vivo data require reliable characterization of the nanoparticles and their aggregates, as well as matrix-based influences on the nanoparticles. Therefore, appropriate analytical techniques should be applied to determine the nanoparticle size distribution, composition, and concentration in the experimental samples.

Widely used methods to detect nanoparticles in liquid dispersions are dynamic light scattering (DLS), centrifugal liquid sedimentation (CLS) (Braun et al. 2011, Cascio et al. 2015, Murdock et al. 2008, Nickel et al. 2014, Powers et al. 2006) and nanoparticle tracking analysis (NTA) (Filipe et al. 2010, Vasco et al. 2010). These methods allow determining an average size or size distribution related to the measured intensity signal. NTA is able to count and size nanoparticles in aqueous media at $\mu \mathrm{g} / \mathrm{L}$ to $\mathrm{mg} / \mathrm{L}$ concentrations (Filipe et al. 2010, Vasco et al. 2010). Both DLS and NTA are highly dependent on the polydispersity of the nanoparticle suspension and material properties of the particles since the scattered light of the individual particles must be sufficiently strong for detection. CLS is more robust since particles are size-separated before their actual detection and sizing. Transmission and scanning electron microscopy (TEM, SEM) are techniques to visualize nanoparticles (Dudkiewicz et al. 2011, Zhang et al. 2012). If pure nanoparticle dispersions are analysed, EM is currently the only technique that reliably covers the entire size range down to $1 \mathrm{~nm}$. In cells or tissues, the minimal particle size that can be detected is around $20 \mathrm{~nm}$, depending on the electron density of the nanoparticles (De Jong et al. 2010). Furthermore, EM distinguishes size aggregates and primary particles. Other imaging techniques are atomic force microscopy (AFM) (Brown et al. 2013) and particle- induced X-ray emission (PIXE) spectroscopy (Lozano et al. 2012, 2013).

Elemental information about the sample can also be obtained by atomic spectrometry methods such as inductively coupled plasma optical emission spectrometry (ICP-OES) (Elzey et al. 2012) and ICP mass spectrometry (ICP-MS) (Krystek 2012, Krystek et al. 2013), especially in single particle mode (sp ICP-MS) (Laborda et al. 2014, Pace et al. 2011, Peters et al. 2014a). From these studies it becomes clear that he smallest particle sizes that now can be determined are around $20 \mathrm{~nm}$ for silver and gold nanoparticles. For $\mathrm{TiO}_{2}$ and $\mathrm{SiO}_{2}$, nanoparticles size detection limits are around 50 and $200 \mathrm{~nm}$; however, recent experiments suggest that the size detection limit may become lower in the next few years. Another promising possibility is to combine size-specific techniques, that separate particles from each other, such as hydrodynamic chromatography (HDC) or field-flow fractionation (FFF) with atomic spectroscopy techniques, that characterize particles, such as ICP-MS (Bednar et al. 2013, Hassellov et al. 
2008, Peters et al. 2014b, Striegel and Brewer 2012, Von der Kammer et al. 2011). Currently, asymmetric flow field-flow fractionation (AF4) is the most successfully used variant of FFF (Zattoni et al. 2014). In in vitro testing, AF4 coupled to ICP-MS becomes a powerful tool to investigate, for example, time-dependent uptake of medium-sized silver nanoparticles (Krystek et al. 2015). Laser ablation (LA)-ICP-MS has been used to quantify gold nanoparticles in single cells (Wang et al. 2014).

Most of the detection methods described above generally require sample preparation procedures. However, only little information concerning sample preparation techniques is available in the literature (Szakal et al. 2014). Aqueous media containing nanoparticles only need limited sample preparation; samples may be sonicated or tip-sonicated to suspend materials and proteins such as bovine serum albumin (BSA), or detergents such as sodium dodecylsulphate (SDS) may be added to stabilize nanoparticle suspensions (Jensen et al. 2011). For other matrices, matrix removal or nanoparticle isolation from the matrix can be achieved by physical processes such as centrifugation, filtration, column techniques or cloud point extraction, or by chemical or enzymatic destruction of the matrix (Loeschner et al. 2014, Peters et al. 2014a, b).

As mentioned not only size, but also, surface chemistry and charge, and composition of the so-called protein corona have been shown to significantly affect the translocation of nanoparticles. The nanoparticle surface charge (or zeta potential) is routinely determined, but a correct interpretation might be hampered by interactions of the matrix with these measurements. More details analysis of the surface of the nanoparticles, for example, by matrix-assisted laser desorption/ionization time-of-flight mass spectrometry (MALDI-TOF) should be considered (Walczak et al.2014). Methods for the assessment of protein composition range from simple gel electrophoreses experiments to a full characterization of the biomolecules that are present using mass spectrometry-based techniques (Lesniak et al. 2010, Lundqvist et al. 2008, Tedja et al. 2012).

Overall, different techniques are available to determine the nanoparticle size distribution, composition and concentration in experimental samples. Attention should be paid to the limits of the chosen techniques and to adequate sample preparation; this should be incorporated in studies reporting translocation data.

\section{Conclusions on comparison of in vitro translocation models with in vivo data}

While comparing the in vitro translocation to in vivo data, we noticed that very few studies tested exactly the same nanoparticles in in vitro and in in vivo settings. It seems that most studies are performed either in vitro or in vivo. Therefore, we focused our comparison on the nanoparticle chemical composition although the nanoparticles studied sometimes differ in 
other particle characteristics such as size. Interestingly, this approach revealed that for some nanoparticles, the in vitro translocation is similar to the in vivo translocation. Examples are the translocation of polystyrene and gold nanoparticles in in vitro lung barrier models, and the translocation of titanium dioxide and silver nanoparticles in in vitro skin models. However, for other nanoparticles, major differences seem to exist between the in vitro translocation rate and the in vivo translocation.

These differences in translocation may be caused by the many differences existing between the in vitro barrier models and the in vivo study designs. First of all, the in vitro models mostly use an acute exposure of maximum $24 \mathrm{~h}$ and a short post-exposure time, while, for example, in vivo inhalation studies vary from $1 \mathrm{~h}$ to 13 weeks of inhalation exposure, 5 days a week, $6 \mathrm{~h}$ per day with post-exposure periods of up to 1 year. The relative short in vitro exposure durations might imply that only a fraction the nanoparticles that have been taken up (intracellularly) has been translocated. Longterm exposures are not conceivable in an in vitro set-up, although this would be a more realistic exposure scenario. Second, exposure concentrations between the in vitro and in vivo studies differ. In in vitro experiments, high particle concentrations are sometimes used to be able to detect the nanoparticles in the basolateral compartment. These high concentrations might increase the agglomeration state of the nanoparticles and damage the epithelial barriers resulting in a different translocation mechanism compared with the in vivo situation. Clearly also in vivo dose selection can be critical, as it has been shown in rats that exposure to high concentrations to silica nanoparticles cause gelation (agglomeration) of silica nanoparticles in the gut (van der Zande et al. 2014). Third, in vitro models have a single basolateral compartment, while in vivo, nanoparticles can translocate to various tissues and organs. In the in vitro set-up, saturation might occur while under in vivo conditions nanoparticles can continue translocating, since they are taken up in organs and removed from the blood. On the other hand, this distribution of nanoparticles to various tissues can hamper translocation studies in vivo. Fourth, current in vitro models are almost all static, while in vivo exposure is dynamic. Fifth, nanoparticles adsorb proteins and/or phospholipids in biological fluids such as serum or lung-lining fluid (Landsiedel et al. 2014b). These proteins or phospholipids form a corona around the particles that affects their uptake and bioavailability (Lesniak et al. 2012). The proteins that are encountered in in vitro models, for example in foetal calf serum, are completely different from the proteins in vivo (i.e. rodent or human). Sixth, many in vitro lung models are submerged, which might alter the nanoparticle characteristics and thus the translocation rate, while most in vivo studies rely on inhalation exposure. 


\section{CONCLUSIONS}

The aim of this review was to evaluate the performance of in vitro models that mimic different physiological barriers found in the human body by comparing - when possible - the in vitro translocation of nanoparticles to their in vivo translocation across the lung, gut, skin, and placental barriers. For all these barriers, a great variety of in vitro models are available to evaluate the translocation of nanoparticles, ranging in complexity from single-cell-type monolayer to multi-cell (3D) models. Many studies that use in vitro models on inserts focus on the toxicity of nanoparticles, do not include their translocation, and were thus not included in this review. Clearly, for a correct interpretation of the observed toxicity, the translocation (or systemic availability, internal concentration) is a crucial parameter. In recent years, the availability of analytical detection methods to quantify and characterize the nanoparticles in in vitro settings has improved considerably, which provides highquality data that are valuable in studying the relationship between physiochemical properties of the nanoparticles and their translocation. The improved analytical chemical detection methods also contributed to an increase in in vivo uptake data (bioavailability) of nanoparticles.

Here, while comparing the in vitro translocation to in vivo data, we noticed that very few studies tested exactly the same materials in both settings. Comparing data obtained using nanoparticles of the same chemical composition, we found that for some nanoparticles, the in vitro translocation is similar to the in vivo bioavailability. Examples are the translocation of polystyrene and gold nanoparticles in in vitro lung barrier models, and that of titanium dioxide and silver nanoparticles in in vitro skin models that are both in line with the in vivo data. However, for other nanoparticles, major differences were found between the in vitro and the in vivo translocation rate. As discussed in the sections above, many differences exist in the experimental set-up between in vitro and in vivo study design that probably account for the poor correlation between these two types of studies. Especially, the changes in the physicochemical characteristics of the nanoparticles caused by the presence of lung-lining fluid, mucus, serum protein, and lipoproteins that form a corona should be taken into account, as these dramatically alter their recognition, uptake and translocation (Lesniak et al. 2012, Treuel et al. 2013).

Risk assessment of nanoparticles (as is true for chemicals in general) still heavily relies on in vivo studies using experimental animals. However, the latter must be reduced as far as possible, for numerous reasons. Therefore, there is an urgent need to validate existing in vitro models using data from animal models, although these animal models do not fully simulate the physiology of humans. How can we establish an in vitro barrier model that has value for the risk assessment of nanoparticles for humans? Ideally, in vitro models reflect the key mechanisms of corresponding in vivo end points, which cannot always be accomplished and 
may not be required if in vitro models reliably detect nanoparticles that are of concern in vivo (Landsiedel et al. 2014b). The predictive value of in vitro models can be better assessed by testing exactly the same nanoparticles simultaneously in both in vitro and in vivo assays. If the ranking of the tested nanoparticles from the lowest translocation rate to the highest translocation rate is the same in both cases, the in vitro models provide information on the internal exposure, which is critical for the ultimate systemic adverse effects. Then, the in vitro models can be considered as suitable for the risk assessment of nanoparticles and will in addition help to reduce animal testing by setting priorities for subsequent in vivo testing.

A crucial factor for both translocation studies is the sensitivity of the analytical technique used for the quantification of the translocation. While this is of importance also for in vivo studies, in vitro studies are more vulnerable for poorly performing methods. The observed low translocation rates combined with the relatively short exposure durations in vitro pose great analytical challenges. The absence of acute in vitro translocation should always be interpreted in relation to the sensitivity of the detection technique (both in terms of concentration and nanoparticle size). The introduction of in vitro models that allow chronic exposures are promising in that a chronic exposure better reflects the real-life human exposure.

In vitro models with a high predictive value do not necessarily have to be complex, but can consist of a single cell type, as long as they give a similar ranking of nanoparticles as obtained in the in vivo situation. However, the mechanism in these models might be completely different from the in vivo situation. To obtain more insight into the mechanisms behind nanoparticle uptake and translocation, in vitro models should be further developed to become physiologically very close to the in vivo human conditions. Such in vitro models must be more complex because the human lung, gut, skin, and placental barrier consist of multiple cell types, which are exposed to a low concentration of nanoparticles. A physiologically realistic model will increase the confidence in the NP testing outcome, but it will also be elaborate and expensive to develop and maintain.

When in vitro models are available that either have a high human predictive value or are physiologically similar to the human situation, experimental data can be used to develop in silico models that will eventually be able to predict the human in vivo bioavailability of nanoparticles from their in vitro translocation rate. The (improved) in vitro models still needs validation, most likely using animal data, clearly alternative approaches need to be developed for this (making use of available human data).

We conclude that the current in vitro models to study the translocation of nanoparticles do not (yet) allow correlating to the reported in vivo translocation because of many differences between the in vitro and in vivo study designs. However, the use of in vitro models is very 
promising since they are currently further improved to mimic the in vivo situation more closely by, for example, using co-cultures of different cell types and implementing them in a microfluidic format. When these models are further validated by testing exactly the same nanoparticles in an in vivo set-up as in the in vitro model, then, they can be used to determine the internal exposure (bioavailability) of nanoparticles and to set priorities for nanoparticles testing.

\section{ACKNOWLEDGEMENTS}

This work is supported by NanoNextNL, a micro- and nanotechnology consortium of the Government of The Netherlands, and 130 partners. 


\section{REFERENCES}

Ali H, Kalashnikova I, White MA, Sherman M, Rytting E (2013) Preparation, characterization, and transport of dexamethasone- loaded polymeric nanoparticles across a human placental in vitro model. Int J Pharm 454(1):149-157. doi:10.1016/j. ijpharm.2013.07.010

Antunes F, Andrade F, Araujo F, Ferreira D, Sarmento B (2013) Establishment of a triple co-culture in vitro cell models to study intestinal absorption of peptide drugs. Eur J Pharm Biopharm 83(3):427-435. doi:10.1016/j. ejpb.2012.10.003

Arai Y, Miyayama T, Hirano S (2014) Difference in the toxicity mechanism between ion and nanoparticle forms of silver in the mouse lung and in macrophages. Toxicology 328:84-92. doi:10.1016/j.tox.2014.12.014

Araujo F, Shrestha N, Shahbazi MA et al (2014) The impact of nanoparticles on the mucosal translocation and transport of GLP-1 across the intestinal epithelium. Biomaterials 35(33):9199- 9207. doi:10.1016/j.biomaterials.2014.07.026

Ardavin C, del Hoyo GM, Martin P et al (2001) Origin and differentiation of dendritic cells. Trends Immunol 22(12):691-700. doi:10.1016/S1471-4906(01)02059-2

Arts JH, Muijser H, Duistermaat E, Junker K, Kuper CF (2007) Five-day inhalation toxicity study of three types of synthetic amorphous silicas in Wistar rats and post-exposure evaluations for up to 3 months. Food Chem Toxicol 45(10):1856-1867. doi:10.1016/j.fct.2007.04.001

Asgharian B, Price O, Miller F et al (2009) Multiple-path particle dosimetry model (MPPD v 2.11): a model for human and rat airway particle dosimetry. In: Applied Research Associates (ARA) HIfHS, National Institute for Public Health and the Environment (RIVM), and Ministry of Housing, Spatial Planning and the Environment (ed). V2.11 edn. Applied Research Associates (ARA), Raleigh, North Carolina

Aye IL, Keelan JA (2013) Placental ABC transporters, cellular toxicity and stress in pregnancy. Chem Biol Interact 203(2):456-466. doi:10.1016/j.cbi.2013.03.007

Bakand S, Hayes A, Dechsakulthorn F (2012) Nanoparticles: a review of particle toxicology following inhalation exposure. Inhal Toxicol 24(2):125-135. doi:10.3109/08958378.2010.642021

Balasubramanian SK, Jittiwat J, Manikandan J, Ong CN, Yu LE, Ong WY (2010) Biodistribution of gold nanoparticles and gene expression changes in the liver and spleen after intravenous administration in rats. Biomaterials 31(8):2034-2042. doi:10.1016/j.biomaterials.2009.11.079

Baroli B (2010) Penetration of nanoparticles and nanomaterials in the skin: fiction or reality? J Pharm Sci 99(1):2150. doi:10.1002/ jps.21817

Bednar AJ, Poda AR, Mitrano DM et al (2013) Comparison of on-line detectors for field flow fractionation analysis of nanomaterials. Talanta 104:140-148. doi:10.1016/j.talanta.2012.11.008

Behrens I, Pena AI, Alonso MJ, Kissel T (2002) Comparative uptake studies of bioadhesive and non-bioadhesive nanoparticles in human intestinal cell lines and rats: the effect of mucus on particle adsorption and transport. Pharm Res 19(8):1185-1193

Bellmann S, Carlander D, Fasano A et al (2015) Mammalian gastrointestinal tract parameters modulating the integrity, surface properties, and absorption of food-relevant nanomaterials. Wiley Interdiscip Rev Nanomed Nanobiotechnol. doi:10.1002/ wnan.1333

Benirschke K, Kaufmann P, Baergen RN (2006) Pathology of the human placenta. Springer, New York

Bermudez LE, Sangari FJ, Kolonoski P, Petrofsky M, Goodman J (2002) The efficiency of the translocation of Mycobacterium tuberculosis across a bilayer of epithelial and endothelial cells as a model of the alveolar wall is a consequence of transport within mononuclear phagocytes and invasion of alveolar epithelial cells. Infect Immun 70(1):140-146

Bhatia SN, Ingber DE (2014) Microfluidic organs-on-chips. Nat Biotechnol 32(8):760-772. doi:10.1038/nbt.2989

Bhattacharjee S, Ershov D, Gucht J et al (2013) Surface charge-specific cytotoxicity and cellular uptake of tri-block copolymer nanoparticles. Nanotoxicology 7(1):71-84. doi:10.3109/17435390.2011.633714

Blank F, Rothen-Rutishauser BM, Schurch S, Gehr P (2006) An optimized in vitro model of the respiratory tract wall to study particle cell interactions. J Aerosol Med 19(3):392-405. doi:10.1089/jam.2006.19.392

Borm PJ, Robbins D, Haubold S et al (2006) The potential risks of nanomaterials: a review carried out for ECETOC. Part Fibre Toxicol 3:11. doi:10.1186/1743-8977-3-11 
Bouwmeester H, Poortman J, Peters RJ et al (2011) Characterization of translocation of silver nanoparticles and effects on whole genome gene expression using an in vitro intestinal epithelium coculture model. ACS Nano 5(5):4091-4103. doi:10.1021/nn2007145

Bouwmeester H, Brandhoff P, Marvin HJ, Weigel S, Peters RJ (2014) State of the safety assessment and current use of nanomaterials in food and food production. Trends Food Sci Technol 40(2):200-210

Braakhuis HM, Gosens I, Krystek P et al (2014a) Particle size dependent deposition and pulmonary inflammation after shortterm inhalation of silver nanoparticles. Part Fibre Toxicol11(1):16. doi:10.1186/s12989-0140049-1

Braakhuis HM, Park MV, Gosens I, De Jong WH, Cassee FR (2014b) Physicochemical characteristics of nanomaterials that affect pulmonary inflammation. Part Fibre Toxicol 11(1):18. doi:10.1186/1743-8977-11-18

Brandenberger C, Rothen-Rutishauser B, Muhlfeld C et al (2010) Effects and uptake of gold nanoparticles deposited at the air-liq- uid interface of a human epithelial airway model. Toxicol Appl Pharmacol 242(1):56-65. doi:10.1016/j.taap.2009.09.014

Braun A, Couteau O, Franks K et al (2011) Validation of dynamic light scattering and centrifugal liquid sedimentation methods for nanoparticle characterisation. Adv Powder Technol 22:766-770

Brown SC, Boyko V, Meyers G, Voetz M, Wohlleben W (2013) Toward advancing nano-object count metrology: a best practice framework. Environ Health Perspect 121(11-12):1282-1291. doi:10.1289/ehp.1306957

Brugmann SA, Wells JM (2013) Building additional complexity to in vitro-derived intestinal tissues. Stem Cell Res Ther 4(Suppl1):S1. doi:10.1186/scrt362

Brun E, Barreau F, Veronesi G et al (2014) Titanium dioxide nanoparticle impact and translocation through ex vivo, in vivo and in vitro gut epithelia. Particle Fibre Toxicol. doi:10.1186/1743-8977-11-13

Buerki-Thurnherr T, von Mandach U, Wick P (2012) Knocking at the door of the unborn child: engineered nanoparticles at the human placental barrier. Swiss Med Wkly 142:w13559. doi:10.4414/ smw.2012.13559

Carr KE, Smyth SH, McCullough MT, Morris JF, Moyes SM (2012) Morphological aspects of interactions between microparticles and mammalian cells: intestinal uptake and onward movement. Prog Histochem Cytochem 46(4):185-252. doi:10.1016/j. proghi.2011.11.001

Cartwright L, Poulsen MS, Nielsen HM et al (2012) In vitro placental model optimization for nanoparticle transport studies. Int J Nanomed 7:497-510. doi:10.2147/ijn.s26601

Carvalho TC, Peters JI, Williams RO 3rd (2011) Influence of particle size on regional lung deposition-what evidence is there? Int J Pharm 406(1-2):1-10. doi:10.1016/j.ijpharm.2010.12.040

Cascio C, Geiss O, Franchini F et al (2015) Detection, quantification and derivation of number size distribution of silver nanoparticles in antimicrobial consumer products. J Anal At Spectrom. doi:10.1039/c4ja00410h

Cevc G, Vierl U (2010) Nanotechnology and the transdermal route: a state of the art review and critical appraisal. J Control Release 141(3):277-299. doi:10.1016/j.jconrel.2009.10.016

Chowdhury F, Howat WJ, Phillips GJ, Lackie PM (2010) Interactions between endothelial cells and epithelial cells in a combined cell model of airway mucosa: effects on tight junction permeability. Exp Lung Res 36(1):1-11. doi:10.3109/01902140903026582

Cone RA (2009) Barrier properties of mucus. Adv Drug Deliv Rev 61(2):75-85. doi:10.1016/j.addr.2008.09.008

Correia Carreira S, Walker L, Paul K, Saunders M (2013) The toxicity, transport and uptake of nanoparticles in the in vitro BeWo b30 placental cell barrier model used within NanoTEST. Nanotoxicology. doi:10.3109/1743 5390.2013.833317

Crater JS, Carrier RL (2010) Barrier properties of gastrointestinal mucus to nanoparticle transport. Macromol Biosci10(12):1473-1483. doi:10.1002/mabi.201000137

Creutzenberg O, Bellmann B, Korolewitz R et al (2012) Change in agglomeration status and toxicokinetic fate of various nanoparticles in vivo following lung exposure in rats. Inhal Toxicol 24(12):821-830. doi:10.3109/0 8958378.2012.721097

Cross SE, Innes B, Roberts MS, Tsuzuki T, Robertson TA, McCormick P (2007) Human skin penetration of sunscreen nanoparticles: in vitro assessment of a novel micronized zinc oxide formulation. Skin Pharmacol Physiol 20(3):148-154. doi:10.1159/000098701

De Jong WH, Hagens WI, Krystek P, Burger MC, Sips AJ, Geertsma RE (2008) Particle size-dependent organ distribution of gold nanoparticles after intravenous administration. Biomaterials 29(12):1912-1919. doi:10.1016/j. biomaterials.2007.12.037 
De Jong WH, Burger MC, Verheijen MA, Geertsma RE (2010) Detection of the presence of gold nanoparticles in organs by transmission electron microscopy. Materials 3:4681-4694

Dekkers S, Krystek P, Peters RJ et al (2011) Presence and risks of nanosilica in food products. Nanotoxicology 5(3):393-405. doi:10.3109/17435390.2010.519836

des Rieux A, Fievez V, Theate I, Mast J, Preat V, Schneider YJ (2007) An improved in vitro model of human intestinal follicle-associated epithelium to study nanoparticle transport by M cells. Eur J Pharm Sci 30(5):380-391. doi:10.1016/j.ejps.2006.12.006

Desforges M, Sibley CP (2010) Placental nutrient supply and fetal growth. Int J Dev Biol 54(2-3):377-390. doi:10.1387/ ijdb.082765md

Di Bona KR, Xu Y, Ramirez PA et al (2014) Surface charge and dosage dependent potential developmental toxicity and biodistribution of iron oxide nanoparticles in pregnant CD-1 mice. Reprod Toxicol 50:36-42. doi:10.1016/j.reprotox.2014.09.010

dos Santos T, Varela J, Lynch I, Salvati A, Dawson KA (2011) Effects of transport inhibitors on the cellular uptake of carboxylated polystyrene nanoparticles in different cell lines. PLoS One 6(9):e24438. doi:10.1371/ journal.pone.0024438

Dudkiewicz A, Tiede K, Loeschner K et al (2011) Characterization of nanomaterials in food by electronmicroscopy. Trends Anal Chem 30:28-43

EFSA (2011) Scientific opinion on guidance on the risk assessment of the application of nanoscience and nanotechnologies in the food and feed chain. EFSA J 9(5):36. doi:10.2903/j.efsa.2011.2140

Elder A, Gelein R, Silva V et al (2006) Translocation of inhaled ultrafine manganese oxide particles to the central nervous system. Environ Health Perspect 114(8):1172-1178

Elzey S, Tsai DH, Rabb SA, Yu LL, Winchester MR, Hackley VA (2012) Quantification of ligand packing density on gold nano- particles using ICP-OES. Anal Bioanal Chem 403(1):145-149. doi:10.1007/s00216-012-5830-0

Fazlollahi F, Sipos A, Kim YH et al (2011) Translocation of PEGylated quantum dots across rat alveolar epithelial cell mon- olayers. Int J Nanomed 6:2849-2857. doi:10.2147/IJN.S26051

Filipe V, Hawe A, Jiskoot W (2010) Critical evaluation of Nanoparticle Tracking Analysis (NTA) by NanoSight for the measurement of nanoparticles and protein aggregates. Pharm Res 27(5):796-810. doi:10.1007/ s11095-010-0073-2

Frieke Kuper C, Grollers-Mulderij M, Maarschalkerweerd T et al (2015) Toxicity assessment of aggregated/agglomerated cerium oxide nanoparticles in an in vitro 3D airway model: the influence of mucociliary clearance. Toxicol In Vitro 29(2):389-397. doi:10.1016/j.tiv.2014.10.017

Frohlich E, Bonstingl G, Hofler A et al (2013) Comparison of two in vitro systems to assess cellular effects of nanoparticles-con- taining aerosols. Toxicol In Vitro 27(1):409-417. doi:10.1016/j. tiv.2012.08.008

Geiser M, Kreyling WG (2010) Deposition and biokinetics of inhaled nanoparticles. Part Fibre Toxicol 7:2. doi:10.1186/1743-8977-7-2

Geiser M, Rothen-Rutishauser B, Kapp N et al (2005) Ultrafine particles cross cellular membranes by nonphagocytic mechanisms in lungs and in cultured cells. Environ Health Perspect 113(11):1555-1560

Genschow E, Spielmann H, Scholz G et al (2002) The ECVAM international validation study on in vitro embryotoxicity tests: results of the definitive phase and evaluation of prediction models. European Centre for the Validation of Alternative Methods. Altern Lab Anim 30(2):151-176

George R, Merten S, Wang TT, Kennedy P, Maitz P (2014) In vivo analysis of dermal and systemic absorption of silver nanoparticles through healthy human skin. Australas J Dermatol 55(3):185-190. doi:10.1111/ajd.12101

George I, Vranic S, Boland S, Courtois A, Baeza-Squiban A (2015) Development of an in vitro model of human bronchial epithelial barrier to study nanoparticle translocation. Toxicol In Vitro 29(1):51-58. doi:10.1016/j. tiv.2014.08.003

Geraets L, Oomen AG, Schroeter JD, Coleman VA, Cassee FR (2012) Tissue distribution of inhaled microand nano-sized cerium oxide particles in rats: results from a 28-day exposure study. Toxicol Sci 127(2):463-473. doi:10.1093/toxsci/kfs113

Geraets L, Oomen AG, Krystek P et al (2014) Tissue distribution and elimination after oral and intravenous administration of different titanium dioxide nanoparticles in rats. Part Fibre Toxicol 11:30. doi:10.1186/1743-8977$11-30$

Geys J, Coenegrachts L, Vercammen J et al (2006) In vitro study of the pulmonary translocation of nanoparticles: a preliminary study. Toxicol Lett 160(3):218-226. doi:10.1016/j.toxlet.2005.07.005 
Geys J, De Vos R, Nemery B, Hoet PH (2009) In vitro translocation of quantum dots and influence of oxidative stress. Am J Physiol Lung Cell Mol Physiol 297(5):L903-L911. doi:10.1152/ ajplung.00029.2009

Grafmuller S, Manser P, Krug HF, Wick P, vonMandach U (2013) Determination of the transport rate of xenobiotics and nanomaterials across the placenta using the ex vivo human placental perfusion model. J Vis Exp. doi:10.3791/50401

Griep LM, Wolbers F, de Wagenaar B et al (2013) BBB on chip: microfluidic platform to mechanically and biochemically modulate blood-brain barrier function. Biomed Microdevices 15(1):145-150. doi:10.1007/s10544012-9699-7

Harink B, Le Gac S, Truckenmuller R, van Blitterswijk C, Habibovic P (2013) Regeneration-on-a-chip? The perspectives on use of microfluidics in regenerative medicine. Lab Chip 13(18):3512-3528. doi:10.1039/c3lc50293g

Hartung T, Balls M, Bardouille C et al (2002) Good cell culture practice. ECVAM good cell culture practice task force report 1. Altern Lab Anim 30(4):407-414

Hartung T, Luechtefeld T, Maertens A, Kleensang A (2013) Integrated testing strategies for safety assessments. ALTEX 30(1):3-18

Hassellov M, Readman JW, Ranville JF, Tiede K (2008) Nanoparticle analysis and characterization methodologies in environmental risk assessment of engineered nanoparticles. Ecotoxicology 17(5):344-361. doi:10.1007/ s10646-008-0225-x

He C, Yin L, Tang C, Yin C (2012) Size-dependent absorption mechanism of polymeric nanoparticles for oral delivery of protein drugs. Biomaterials 33(33):8569-8578. doi:10.1016/j. biomaterials.2012.07.063

Helbig A, Silletti E, van Aken GA et al (2013) Lipid digestion of protein stabilized emulsions investigated in a dynamic in vitro gastro-intestinal model system. Food Dig 4:58-68

Hermanns MI, Unger RE, Kehe K, Peters K, Kirkpatrick CJ (2004) Lung epithelial cell lines in coculture with human pulmonary microvascular endothelial cells: development of an alveolo-capillary barrier in vitro. Lab Invest 84(6):736-752. doi:10.1038/ labinvest.3700081

Hermanns MI, Kasper J, Dubruel P, Pohl C, Uboldi C, Vermeersch V, Fuchs S, Unger RE, Kirkpatrick CJ (2010) An impaired alveolar-capillary barrier in vitro: effect of proinflammatory cytokines and consequences on nanocarrier interaction. J R Soc Interface 7(Suppl 1):S41-S54. doi:10.1098/rsif.2009.0288. focus

Herzog F, Clift MJ, Piccapietra F et al (2013) Exposure of silver-nanoparticles and silver-ions to lung cells in vitro at the air-liquid interface. Part Fibre Toxicol 10(1):11. doi:10.1186/1743-8977-10-11

Hillery AM, Florence AT (1996) The effect of adsorbed poloxamer188 and 407 surfactants on the intestinal uptake of 60 -nm polystyrene particles after oral administration in the rat. Int J Pharm 132(1-2):123-130. doi:10.1016/0378-5173(95)04353-5

Hillery AM, Jani PU, Florence AT (1994) Comparative, quantitative study of lymphoid and non-lymphoid uptake of $60 \mathrm{~nm}$ polystyrene particles. J Drug Target 2(2):151-156. doi:10.3109/10611869409015904

Hillyer JF, Albrecht RM (2001) Gastrointestinal persorption and tissue distribution of differently sized colloidal gold nanoparticles. J Pharm Sci 90(12):1927-1936

Holder AL, Marr LC (2013) Toxicity of silver nanoparticles at the air-liquid interface. Biomed Res Int 2013:328934. doi:10.1155/2013/328934

Huh D, Fujioka H, Tung YC et al (2007) Acoustically detectable cellular-level lung injury induced by fluid mechanical stresses in microfluidic airway systems. Proc Natl Acad Sci USA 104(48):18886-18891. doi:10.1073/ pnas.0610868104

Huh D, Matthews BD, Mammoto A, Montoya-Zavala M, Hsin HY, Ingber DE (2010) Reconstituting organ-level lung functions on a chip. Science 328(5986):1662-1668. doi:10.1126/ science.1188302

Huh D, Torisawa YS, Hamilton GA, Kim HJ, Ingber DE (2012) Microengineered physiological biomimicry: organs-on-chips. Lab Chip 12(12):2156-2164. doi:10.1039/c2lc40089h

Huh D, Kim HJ, Fraser JP et al (2013) Microfabrication of human organs-on-chips. Nat Protoc 8(11):2135-2157. doi:10.1038/ nprot.2013.137

Hussain N, Florence AT (1998) Utilizing bacterial mechanisms of epithelial cell entry: invasin-induced oral uptake of latex nanoparticles. Pharm Res 15(1):153-156

Hussain N, Jani PU, Florence AT (1997) Enhanced oral uptake of tomato lectin-conjugated nanoparticles in the rat. Pharm Res14(5):613-618

Hussain N, Jaitley V, Florence AT (2001) Recent advances in the understanding of uptake of microparticulates across the gastrointestinal lymphatics. Adv Drug Deliv Rev 50(1-2):107-142 
ICRP (1994) Human respiratory tract model for radiological protection. ICRP Publication 66, vol 24. International Commission on Radiological Protection, p 1-3

Ingels F, Deferme S, Destexhe E, Oth M, Van den Mooter G, Augustijns P (2002) Simulated intestinal fluid as transport medium in the Caco-2 cell culture model. Int J Pharm 232(1-2):183-192

Jakasa I, Kezic S (2008) Evaluation of in vivo animal and in vitro models for prediction of dermal absorption in man. Hum Exp Toxicol 27(4):281-288. doi:10.1177/0960327107085826

Janer G, Mas del Molino E, Fernandez-Rosas E, Fernandez A, Vazquez-Campos S (2014) Cell uptake and oral absorption of titanium dioxide nanoparticles. Toxicol Lett 228(2):103-110. doi:10.1016/j.toxlet.2014.04.014

Jani P, Halbert GW, Langridge J, Florence AT (1989) The uptake and translocation of latex nanospheres and microspheres after oral administration to rats. J Pharm Pharmacol 41(12):809-812

Jani P, Halbert GW, Langridge J, Florence AT (1990) Nanoparticle uptake by the rat gastrointestinal mucosa: quantitation and particle size dependency. J Pharm Pharmacol 42(12):821-826

Jensen KA, Kembouche Y, Christiansen E et al (2011) Final protocol for producing suitable manufactures nanomaterial exposure media. The generic NANOGENOTOX dispersion protocol, Standard Operation Procedure (SOP). http://www.nanogenotox. eu/

Jin Y, Song Y, Zhu X et al (2012) Goblet cell-targeting nanoparticles for oral insulin delivery and the influence of mucus on insulin transport. Biomaterials 33(5):1573-1582. doi:10.1016/j. biomaterials.2011.10.075

Kadiyala I, Loo Y, Roy K, Rice J, Leong KW (2010) Transport of chitosan-DNA nanoparticles in human intestinal M-cell model versus normal intestinal enterocytes. Eur J Pharm Sci 39(1-3):103-109. doi:10.1016/j. ejps.2009.11.002

Kandarova H, Letasiova S (2011) Alternative methods in toxicology: pre-validated and validated methods. Interdiscip Toxicol4(3):107-113. doi:10.2478/v10102-011-0018-6

Kerneis S, Bogdanova A, Kraehenbuhl JP, Pringault E (1997) Conversion by Peyer's patch lymphocytes of human enterocytes into M cells that transport bacteria. Science 277(5328):949-952

Kezic S, Nielsen JB (2009) Absorption of chemicals through compromised skin. Int Arch Occup Environ Health 82(6):677-688. doi:10.1007/s00420-009-0405-x

Kezic S, Novak N, Jakasa I et al (2014) Skin barrier in atopic dermatitis. Front Biosci (Landmark Ed) 19:542-556

Kim HJ, Ingber DE (2013) Gut-on-a-Chip microenvironment induces human intestinal cells to undergo villus differentiation. Integr Biol (Camb) 5(9):1130-1140. doi:10.1039/c3ib40126j

Klein SG, Hennen J, Serchi T, Blomeke B, Gutleb AC (2011) Potential of coculture in vitro models to study inflammatory and sensitizing effects of particles on the lung. Toxicol In Vitro 25(8):1516-1534. doi:10.1016/j. tiv.2011.09.006

Kong F, Singh RP (2010) A human gastric simulator (HGS) to study food digestion in human stomach. J Food Sci 75(9):E627-E635. doi:10.1111/j.1750-3841.2010.01856.x

Kreyling WG, Semmler-Behnke M, Seitz J et al (2009) Size dependence of the translocation of inhaled iridium and carbon nanoparticle aggregates from the lung of rats to the blood and secondary target organs. Inhal Toxicol 21(Suppl 1):55-60. doi:10.1080/08958370902942517

Krystek P (2012) A review on approaches to biodistribution studies about gold and silver engineered nanoparticles by inductively couples plasma mass spectrometry. Microchem J 105:39-43. doi:10.1016/j.microc.2012.02.008

Krystek P, Braakhuis HM, Park MVDZ, Jong WHd (2013) Inductively coupled plasma-mass spectrometry in biodistribution studies of (Engineered) nanoparticles encyclopaedia of analytical chemistry. Wiley, New York

Krystek P, Kettler K, van der Wagt B, De Jong WH (2015) Exploring influences on the cellular uptake of medium-sized silver nanoparticles into THP-1 cells. Microchem J 120:45-50

Kulvietis V, Zalgeviciene V, Didziapetriene J, Rotomskis R (2011) Transport of nanoparticles through the placental barrier. Tohoku J Exp Med 225(4):225-234

Laborda F, Bolea E, Jimenez-Lamana J (2014) Single particle inductively coupled plasma mass spectrometry: a powerful tool for nanoanalysis. Anal Chem 86(5):2270-2278. doi:10.1021/ ac402980q

Labouta HI, Schneider M (2013) Interaction of inorganic nanoparticles with the skin barrier: current status and critical review. Nanomedicine 9(1):39-54. doi:10.1016/j.nano.2012.04.004

Labouta HI, Liu DC, Lin LL et al (2011a) Gold nanoparticle penetration and reduced metabolism in human skin by toluene. Pharm Res 28(11):2931-2944. doi:10.1007/s11095-011-0561-z

Labouta HI, el-Khordagui LK, Kraus T, Schneider M (2011b) Mechanism and determinants of nanoparticle penetration through human skin. Nanoscale 3(12):4989-4999. doi:10.1039/ clnr11109d 
Lager S, Powell TL (2012) Regulation of nutrient transport across the placenta. J Pregnancy 2012:179827. doi:10.1155/2012/179827

Lai SK, O'Hanlon DE, Harrold S et al (2007) Rapid transport of large polymeric nanoparticles in fresh undiluted human mucus. Proc Natl Acad Sci USA 104(5):1482-1487. doi:10.1073/ pnas.0608611104

Landsiedel R, Ma-Hock L, Hofmann T et al (2014a) Application of short-term inhalation studies to assess the inhalation toxicity of nanomaterials. Part Fibre Toxicol 11:16. doi:10.1186/1743-8977-11-16

Landsiedel R, Sauer UG, Ma-Hock L, Schnekenburger J, Wiemann M (2014b) Pulmonary toxicity of nanomaterials: a critical comparison of published in vitro assays and in vivo inhalation or instillation studies. Nanomedicine (Lond) 9(16):2557-2585. doi:10.2217/nnm.14.149

Larese FF, D'Agostin F, Crosera M et al (2009) Human skin penetration of silver nanoparticles through intact and damaged skin. Toxicology 255(1-2):33-37. doi:10.1016/j.tox.2008.09.025

Lefebvre DE, Venema K, Gombau L et al (2014) Utility of models of the gastrointestinal tract for assessment of the digestion and absorption of engineered nanomaterials released from food matrices. Nanotoxicology. doi: 1 0.3109/17435390.2014.948091

Lehmann AD, Daum N, Bur M, Lehr CM, Gehr P, Rothen-Rut- ishauser BM (2011) An in vitro triple cell co-culture model with primary cells mimicking the human alveolar epithelial barrier. Eur J Pharm Biopharm 77(3):398-406. doi:10.1016/j. ejpb.2010.10.014

Leite-Silva VR, Le Lamer M, Sanchez WY et al (2013) The effect of formulation on the penetration of coated and uncoated zinc oxide nanoparticles into the viable epidermis of human skin in vivo. Eur J Pharm Biopharm 84(2):297-308. doi:10.1016/j. ejpb.2013.01.020

Lenz AG, Karg E, Lentner B et al (2009) A dose-controlled system for air-liquid interface cell exposure and application to zinc oxide nanoparticles. Part Fibre Toxicol 6:32. doi:10.1186/1743-8977-6-32

Lenz AG, Karg E, Brendel E et al (2013) Inflammatory and oxidative stress responses of an alveolar epithelial cell line to airborne zinc oxide nanoparticles at the air-liquid interface: a comparison with conventional, submerged cell-culture conditions. Biomed Res Int 2013:652632. doi:10.1155/2013/652632

Lesniak A, Campbell A, Monopoli MP, Lynch I, Salvati A, Dawson KA (2010) Serum heat inactivation affects protein corona composition and nanoparticle uptake. Biomaterials 31(36):9511-9518. doi:10.1016/j.biomaterials.2010.09.049

Lesniak A, Fenaroli F, Monopoli MP, Aberg C, Dawson KA, Salvati A (2012) Effects of the presence or absence of a protein corona on silica nanoparticle uptake and impact on cells. ACS Nano 6(7):5845-5857. doi:10.1021/ nn300223w

Li H, van Ravenzwaay B, Rietjens IM, Louisse J (2013) Assessment of an in vitro transport model using BeWo b30 cells to predict placental transfer of compounds. Arch Toxicol 87(9):1661-1669. doi:10.1007/s00204013-1074-9

Loeschner K, Brabrand MS, Sloth JJ, Larsen EH (2014) Use of alkaline or enzymatic sample pretreatment prior to characterization of gold nanoparticles in animal tissue by single-particle ICPMS. Anal Bioanal Chem 406(16):3845-3851. doi:10.1007/ s00216-013-7431-y

Lozano O, Mejia J, Masereel B, Toussaint O, Lison D, Lucas S (2012) Development of a PIXE analysis method for the determination of the biopersistence of $\mathrm{SiC}$ and $\mathrm{TiC}$ nanoparticles in rat lungs. Nanotoxicology 6(3):263271. doi:10.3109/17435390.2011.572301

Lozano O, Olivier T, Dogne JM, Lucas S (2013) The use of PIXE for engineered nanomaterials quantification in complex matrices. J Phys Conf Ser 429:012010

Lundqvist M, Stigler J, Elia G, Lynch I, Cedervall T, Dawson KA (2008) Nanoparticle size and surface properties determine the protein corona with possible implications for biological impacts. Proc Natl Acad Sci USA 105(38):14265-14270. doi:10.1073/pnas.0805135105

MacNicoll A, Kelly M, Aksoy H, Kramer E, Bouwmeester H, Chaudhry Q (2015) A study of the uptake and biodistribution of nano-titanium dioxide using in vitro and in vivo models of oral intake. J Nanopart Res 17(66):20

Madlova M, Jones SA, Zwerschke I, Ma Y, Hider RC, Forbes B (2009) Poly(vinyl alcohol) nanoparticle stability in biological media and uptake in respiratory epithelial cell layers in vitro. Eur J Pharm Biopharm 72(2):437443. doi:10.1016/j. ejpb.2009.01.009

Mahler GJ, Esch MB, Glahn RP, Shuler ML (2009) Characterization of a gastrointestinal tract microscale cell culture analog used to predict drug toxicity. Biotechnol Bioeng 104(1):193-205. doi:10.1002/bit.22366 
Mahler GJ, Esch MB, Tako E et al (2012) Oral exposure to polystyrene nanoparticles affects iron absorption. Nat Nanotechnol 7(4):264-271. doi:10.1038/nnano.2012.3

Ma-Hock L, Brill S, Wohlleben W et al (2012) Short term inhalation toxicity of a liquid aerosol of CdS/Cd(OH) core shell quan- tum dots in male Wistar rats. Toxicol Lett 208(2):115-124. doi:10.1016/j.toxlet.2011.10.011

Makhlof A, Werle M, Tozuka Y, Takeuchi H (2011) A mucoadhesive nanoparticulate system for the simultaneous delivery of macromolecules and permeation enhancers to the intestinal mucosa. J Control Release 149(1):81-88. doi:10.1016/j. jconrel.2010.02.001

Martinez-Argudo I, Sands C, Jepson MA (2007) Translocation of enteropathogenic Escherichia coli across an in vitro $\mathrm{M}$ cell model is regulated by its type III secretion system. Cell Microbiol 9(6):1538-1546. doi:10.1111/ j.1462-5822.2007.00891.x

Marx U, Walles H, Hoffmann S et al (2012) 'Human-on-a-chip' developments: a translational cutting-edge alternative to systemic safety assessment and efficiency evaluation of substances in laboratory animals and man? Altern Lab Anim 40(5):235-257

Mavon A, Miquel C, Lejeune O, Payre B, Moretto P (2007) In vitro percutaneous absorption and in vivo stratum corneum distribution of an organic and a mineral sunscreen. Skin Pharmacol Physiol 20(1):10-20. doi:10.1159/000096167

Menjoge AR, Rinderknecht AL, Navath RS et al (2011) Transfer of PAMAM dendrimers across human placenta: prospects of its use as drug carrier during pregnancy. J Control Release 150(3):326-338. doi:10.1016/j. jconrel.2010.11.023

Minekus M, Marteau P, Havenaar R, Huis in’t Veld JHJ (1995) A multi compartmental dynamic computer-controlled model simulating the stomach and small intestine. Altern Lab Anim (ATLA) 23:197-209

Minekus M, Alminger M, Alvito $\mathrm{P}$ et al (2014) A standardised static in vitro digestion method suitable for food-an international consensus. Food Funct 5(6):1113-1124. doi:10.1039/ c3fo60702j

Miquel-Jeanjean C, Crepel F, Raufast V et al (2012) Penetration study of formulated nanosized titanium dioxide in models of damaged and sun-irradiated skins. Photochem Photobiol 88(6):1513-1521. doi:10.1111/ j.1751-1097.2012.01181.x

Miret S, Abrahamse L, de Groene EM (2004) Comparison of in vitro models for the prediction of compound absorption across the human intestinal mucosa. J Biomol Screen 9(7):598-606. doi:10.1177/1087057104267162

Möller W, Kreyling WG, Schmid O, Semmler-Behnke M, Schulz H (2010) Deposition, retention and clearance, and translocation of inhaled fine and nano-sized particles in the respiratory tract. In: Gehr P, Mühlfeld C, Rothen-Rutishauser B, Blank F (eds) Particle-Lung Interactions, 2nd edn. Informa Healthcare USA Inc, New York, p 338

Monteiro-Riviere NA, Wiench K, Landsiedel R, Schulte S, Inman AO, Riviere JE (2011) Safety evaluation of sunscreen formulations containing titanium dioxide and zinc oxide nanoparticles in UVB sunburned skin: an in vitro and in vivo study. Toxicol Sci 123(1):264-280. doi:10.1093/toxsci/kfr148

Moraes C, Mehta G, Lesher-Perez SC, Takayama S (2012) Organs- on-a-chip: a focus on compartmentalized microdevices. Ann Biomed Eng 40(6):1211-1227. doi:10.1007/s10439-011-0455-6

Muhlfeld C, Gehr P, Rothen-Rutishauser B (2008) Translocation and cellular entering mechanisms of nanoparticles in the respiratory tract. Swiss Med Wkly 138(27-28):387-391

Muller L, Riediker M, Wick P, Mohr M, Gehr P, Rothen-Rutishauser B (2010) Oxidative stress and inflammation response after nanoparticle exposure: differences between human lung cell monocultures and an advanced three-dimensional model of the human epithelial airways. J R Soc Interface 7(Suppl 1):S27- S40. doi:10.1098/rsif.2009.0161.focus

Murdock RC, Braydich-Stolle L, Schrand AM, Schlager JJ, Hussain SM (2008) Characterization of nanomaterial dispersion in solution prior to in vitro exposure using dynamic light scattering technique. Toxicol Sci 101(2):239-253. doi:10.1093/toxsci/kfm240

Mwilu SK, El Badawy AM, Bradham K et al (2013) Changes in silver nanoparticles exposed to human synthetic stomach fluid: effects of particle size and surface chemistry. Sci Total Environ 447:90-98. doi:10.1016/j. scitotenv.2012.12.036

Myllynen PK, Loughran MJ, Howard CV, Sormunen R, Walsh AA, Vahakangas KH (2008) Kinetics of gold nanoparticles in the human placenta. Reprod Toxicol 26(2):130-137. doi:10.1016/j. reprotox.2008.06.008 
Nalayanda DD, Wang Q, Fulton WB, Wang TH, Abdullah F (2010) Engineering an artificial alveolar-capillary membrane: a novel continuously perfused model within microchannels. J Pediatr Surg 45(1):45-51. doi:10.1016/j. jpedsurg.2009.10.008

Nanotechnologies PoE (2014) Consumer Products Inventory. http://www.nanotechproject.org/cpi. Accessed June 2014

Natoli M, Leoni BD, D’Agnano I, Zucco F, Felsani A (2012) Good Caco-2 cell culture practices. Toxicol In Vitro 26(8):1243-1246. doi:10.1016/j.tiv.2012.03.009

Newsome R (2014) 2013 IFT International Food Nanoscience Conference: proceedings. Compr Rev Food Sci Food Saf 13(2):190-228. doi:10.1111/1541-4337.12055

Nickel C, Angelstorf J, Bienert R et al (2014) Dynamic light-scattering measurement comparability of nanomaterial suspensions. J Nanopart Res 16:1-12

Nkabinde LA, Shoba-Zikhali LN, Semete-Makokotlela B et al (2012) Permeation of PLGA nanoparticles across different in vitro models. Curr Drug Deliv 9(6):617-627

Norris DA, Puri N, Sinko PJ (1998) The effect of physical barriers and properties on the oral absorption of particulates. Adv Drug Deliv Rev 34(2-3):135-154

Oberdorster G (1989) Dosimetric principles for extrapolating results of rat inhalation studies to humans, using an inhaled Ni compound as an example. Health Phys 57(Suppl 1):213-220

Oberdorster G, Ferin J, Lehnert BE (1994) Correlation between particle size, in vivo particle persistence, and lung injury. Environ Health Perspect 102(Suppl 5):173-179

Oberdorster G, Sharp Z, Atudorei V et al (2004) Translocation of inhaled ultrafine particles to the brain. Inhal Toxicol 16(6-7):437-445. doi:10.1080/08958370490439597

OECD (2004) Guidelines for the testing of chemicals, section 4. Test no.428: skin absorption: in vitro method

Oomen AG, Tolls J, Sips AJ, Van den Hoop MA (2003) Lead speciation in artificial human digestive fluid. Arch Environ Contam Toxicol 44(1):107-115. doi:10.1007/s00244-002-1225-0

Pace HE, Rogers NJ, Jarolimek C, Coleman VA, Higgins CP, Ranville JF (2011) Determining transport efficiency for the purpose of counting and sizing nanoparticles via single particle inductively coupled plasma mass spectrometry. Anal Chem 83(24):9361-9369. doi:10.1021/ac201952t

Papritz M, Pohl C, Wübbeke C, Moisch M, Hofmann H, Hermanns MI, Thiermann H, Kirkpatrick CJ, Kehe K (2010) Side-specific effects by cadmium exposure: apical and basolateral treatment in a coculture model of the blood-air barrier. Toxicol Appl Pharmacol 245(3):361-369. doi:10.1016/j. taap.2010.04.002

Paranjpe M, Muller-Goymann CC (2014) Nanoparticle-mediated pulmonary drug delivery: a review. Int J Mol Sci 15(4):5852-5873. doi:10.3390/ijms15045852

Peters R, Kramer E, Oomen AG et al (2012) Presence of nano-sized silica during in vitro digestion of foods containing silica as a food additive. ACS Nano 6(3):2441-2451. doi:10.1021/nn204728k

Peters RJ, Rivera ZH, van Bemmel G, Marvin HJ, Weigel S, Bouwmeester H (2014a) Development and validation of single particle ICP-MS for sizing and quantitative determination of nano- silver in chicken meat. Anal Bioanal Chem 406(16):3875-3885. doi:10.1007/s00216-013-7571-0

Peters RJ, van Bemmel G, Herrera-Rivera Z et al (2014b) Characterization of titanium dioxide nanoparticles in food products: analytical methods to define nanoparticles. J Agric Food Chem 62(27):6285-6293. doi:10.1021/ jf5011885

Phalen RF, Mendez LB, Oldham MJ (2010) New developments in aerosol dosimetry. Inhal Toxicol 22(Suppl 2):6-14. doi:10.3109/08958378.2010.516031

Pilcer G, Amighi K (2010) Formulation strategy and use of excipients in pulmonary drug delivery. Int J Pharm 392(1-2):1-19. doi:10.1016/j.ijpharm.2010.03.017

Poulsen MS, Rytting E, Mose T, Knudsen LE (2009) Modeling placental transport: correlation of in vitro BeWo cell permeability and ex vivo human placental perfusion. Toxicol In Vitro 23(7):1380-1386. doi:10.1016/j. tiv.2009.07.028

Powell JJ, Ainley CC, Harvey RS et al (1996) Characterisation of inorganic microparticles in pigment cells of human gut associated lymphoid tissue. Gut 38(3):390-395

Powell JJ, Faria N, Thomas-McKay E, Pele LC (2010) Origin and fate of dietary nanoparticles and microparticles in the gastrointestinal tract. J Autoimmun 34(3):J226-J233. doi:10.1016/j. jaut.2009.11.006 
Powers KW, Brown SC, Krishna VB, Wasdo SC, Moudgil BM, Roberts SM (2006) Research strategies for safety evaluation of nanomaterials. Part VI. Characterization of nanoscale particles for toxicological evaluation. Toxicol Sci 90(2):296-303. doi:10.1093/toxsci/kfj099

Prow TW, Monteiro-Riviere NA, Inman AO et al (2012) Quantum dot penetration into viable human skin. Nanotoxicology 6(2):173-185. doi:10.3109/17435390.2011.569092

Raemy DO, Limbach LK, Rothen-Rutishauser B et al (2011) Cerium oxide nanoparticle uptake kinetics from the gas-phase into lung cells in vitro is transport limited. Eur J Pharm Biopharm 77(3):368-375. doi:10.1016/j. ejpb.2010.11.017

Rieux A, Ragnarsson EG, Gullberg E, Preat V, Schneider YJ, Artursson P (2005) Transport of nanoparticles across an in vitro model of the human intestinal follicle associated epithelium. Eur J Pharm Sci 25(4-5):455-465. doi:10.1016/j.ejps.2005.04.015

Rothen-Rutishauser BM, Kiama SG, Gehr P (2005) A three-dimensional cellular model of the human respiratory tract to study the interaction with particles. Am J Respir Cell Mol Biol 32(4):281-289

Rothen-Rutishauser B, Mueller L, Blank F, Brandenberger C, Muehlfeld C, Gehr P (2008) A newly developed in vitro model of the human epithelial airway barrier to study the toxic potential of nanoparticles. ALTEX 25(3):191-196

Rothen-Rutishauser B, Grass RN, Blank F et al (2009) Direct combination of nanoparticle fabrication and exposure to lung cell cultures in a closed setup as a method to simulate accidental nanoparticle exposure of humans. Environ Sci Technol 43(7):2634-2640

Sadauskas E, Jacobsen NR, Danscher G et al (2009) Biodistribution of gold nanoparticles in mouse lung following intratracheal instillation. Chem Cent J 3:16. doi:10.1186/1752-153X-3-16

Sarlo K, Blackburn KL, Clark ED et al (2009) Tissue distribution of $20 \mathrm{~nm}, 100 \mathrm{~nm}$ and $1000 \mathrm{~nm}$ fluorescent polystyrene latex nanospheres following acute systemic or acute and repeat airway exposure in the rat. Toxicology 263(2-3):117-126. doi:10.1016/j.tox.2009.07.002

Saunders M (2009) Transplacental transport of nanomaterials. Wiley Interdiscip Rev Nanomed Nanobiotechnol 1(6):671-684. doi:10.1002/wnan.53

Savi M, Kalberer M, Lang D et al (2008) A novel exposure system for the efficient and controlled deposition of aerosol particles onto cell cultures. Environ Sci Technol 42(15):5667-5674

Scaldaferri F, Pizzoferrato M, Gerardi V, Lopetuso L, Gasbarrini A (2012) The gut barrier: new acquisitions and therapeutic approaches. J Clin Gastroenterol 46(Suppl):S12-S17. doi:10.1097/MCG.0b013e31826ae849

Schimek K, Busek M, Brincker S et al (2013) Integrating biological vasculature into a multi-organ-chip microsystem. Lab Chip 13(18):3588-3598. doi:10.1039/c3lc50217a

Schimpel C, Teubl B, Absenger M et al (2014) Development of an advanced intestinal in vitro triple culture permeability model to study transport of nanoparticles. Mol Pharm 11(3):808-818. doi:10.1021/mp400507g

Seifert J, Haraszti B, Sass W (1996) The influence of age and particle number on absorption of polystyrene particles from the rat gut. J Anat 189:483-486

Semmler M, Seitz J, Erbe F et al (2004) Long-term clearance kinetics of inhaled ultrafine insoluble iridium particles from the rat lung, including transient translocation into secondary organs. Inhal Toxicol 16(6-7):453-459. doi:10.1080/08958370490439650

Sinha R, Le Gac S, Verdonschot N, van den Berg A, Koopman B, Rouwkema J (2015) A medium throughput device to study the effects of combinations of surface strains and fluid-flow shear stresses on cells. Lab Chip 15(2):429-439. doi:10.1039/ c4lc01259c

Sonnegaard Poulsen M, Mose T, Leth Maroun L, Mathiesen L, Ehlert Knudsen L, Rytting E (2013) Kinetics of silica nanoparticles in the human placenta. Nanotoxicology. doi:10.3109/17435390.2013.812259

Striegel AM, Brewer AK (2012) Hydrodynamic chromatography.Annu Rev Anal Chem (Palo Alto Calif) 5:15-34. doi:10.1146/ annurev-anchem-062011-143107

Sung JH, Ji JH, Park JD et al (2011) Subchronic inhalation toxicity of gold nanoparticles. Part Fibre Toxicol 8:16. doi:10.1186/1743-8977-8-16

Szakal C, Roberts SM, Westerhoff P et al (2014) Measurement of nanomaterials in foods: integrative consideration of challenges and future prospects. ACS Nano 8(4):3128-3135. doi:10.1021/ nn501108g

Szentkuti L, Lorenz K (1995) The thickness of the mucus layer in different segments of the rat intestine. Histochem J 27(6):466-472 
Takahashi S, Matsuoka O (1981) Cross placental transfer of 198Au colloid in near term rats. J Radiat Res 22(2):242249

Takenaka S, Karg E, Roth C et al (2001) Pulmonary and systemic distribution of inhaled ultrafine silver particles in rats. Environ Health Perspect 109(Suppl 4):547-551

Takenaka S, Karg E, Kreyling WG et al (2006) Distribution pattern of inhaled ultrafine gold particles in the rat lung. Inhal Toxicol 18(10):733-740. doi:10.1080/08958370600748281

Tassinari R, Cubadda F, Moracci G et al (2014) Oral, short-term exposure to titanium dioxide nanoparticles in Sprague-Dawley rat: focus on reproductive and endocrine systems and spleen. Nanotoxicology 8(6):654662. doi:10.3109/17435390.2013.822114

Taylor AJ, McClure CD, Shipkowski KA et al (2014) Atomic layer deposition coating of carbon nanotubes with aluminum oxide alters pro-fibrogenic cytokine expression by human mononuclear phagocytes in vitro and reduces lung fibrosis in mice in vivo. PLoS One 9(9):e106870. doi:10.1371/journal.pone.0106870

Tedja R, Lim M, Amal R, Marquis C (2012) Effects of serum adsorption on cellular uptake profile and consequent impact of titanium dioxide nanoparticles on human lung cell lines. ACS Nano 6(5):4083-4093. doi:10.1021/ nn3004845

Tian F, Razansky D, Estrada GG et al (2009) Surface modification and size dependence in particle translocation during early embryonic development. Inhal Toxicol 21(Suppl 1):92-96. doi:10.1080/08958370902942624

Treuel L, Jiang X, Nienhaus GU (2013) New views on cellular uptake and trafficking of manufactured nanoparticles. J R Soc Interface 10(82):20120939. doi:10.1098/rsif.2012.0939

van de Stolpe A, den Toonder J (2013) Workshop meeting report Organs-on-Chips: human disease models. Lab Chip 13(18):3449-3470. doi:10.1039/c3lc50248a

Van de Wiele TR, Oomen AG, Wragg J et al (2007) Comparison of five in vitro digestion models to in vivo experimental results: lead bioaccessibility in the human gastrointestinal tract. J Environ Sci Health A Tox Hazard Subst Environ Eng 42(9):1203-1211. doi:10.1080/10934520701434919

van der Meer AD, van den Berg A (2012) Organs-on-chips: breaking the in vitro impasse. Integr Biol (Camb) 4(5):461-470. doi:10.1039/c2ib00176d

van der Zande M, Vandebriel RJ, Van Doren E et al (2012) Distribution, elimination, and toxicity of silver nanoparticles and silver ions in rats after 28-day oral exposure. ACS Nano 6(8):7427-7442. doi:10.1021/nn302649p

van der Zande M, Vandebriel RJ, Groot MJ et al (2014) Sub-chronic toxicity study in rats orally exposed to nanostructured silica. Part Fibre Toxicol 11:8. doi:10.1186/1743-8977-11-8

van Kesteren PCE, Cubadda F, Bouwmeester H et al (2014) Novel insights into the risk assessment of the nanomaterial synthetic amorphous silica, additive E551, in food. Nanotoxicology. doi:10.3109/17435390.2014.940408

Vandebriel RJ, De Jong WH (2012) A review of mammalian toxicity of ZnO nanoparticles. Nanotechnol Sci Appl 5:61-71. doi:10.2147/nsa.s23932

VanDussen KL, Marinshaw JM, Shaikh N et al (2014) Development of an enhanced human gastrointestinal epithelial culture system to facilitate patient-based assays. Gut. doi:10.1136/ gutjnl-2013-306651

Vasco F, Hawe A, Jiskoot W (2010) Critical evaluation of nanoparticle tracking analysis (NTA) by nanosight for the measurement of nanoparticles and protein aggregates. Pharm Res 27:796-810

Versantvoort CH, Oomen AG, Van de Kamp E, Rompelberg CJ, Sips AJ (2005) Applicability of an in vitro digestion model in assessing the bioaccessibility of mycotoxins from food. Food Chem Toxicol 43(1):31-40. doi:10.1016/j.fct.2004.08.007

Von der Kammer F, Legros S, Hofmann T, Larsen EH, Loeschner K (2011) Separation characterization of nanoparticles in complex food and environmental samples by field-flow fractionation. Trends Anal Chem 30:425436

Wagner I, Materne EM, Brincker S et al (2013) A dynamic multi- organ-chip for long-term cultivation and substance testing proven by 3D human liver and skin tissue co-culture. Lab Chip 13(18):3538-3547. doi:10.1039/ c3lc50234a

Walczak AP (2015) Development of an integrated in vitro model for the prediction of oral bioavailability of nanoparticles. Wageningen University, Wageningen.

Walczak AP, Fokkink R, Peters R et al (2013) Behaviour of silver nanoparticles and silver ions in an in vitro human gastrointestinal digestion model. Nanotoxicology 7(7):1198-1210. doi:10.3109/17435390.2012.726382 
Walczak AP, Kramer E, Hendriksen PJ et al (2014) Translocation of differently sized and charged polystyrene nanoparticles in in vitro intestinal cell models of increasing complexity. Nanotoxicology. doi:10.3109/1743 5390.2014.944599

Walczak AP, Kramer E, Hendriksen PJ et al (2015) In vitro gastrointestinal digestion increases the translocation of polystyrene nanoparticles in an in vitro intestinal co-culture model. Nanotoxicology. doi:10.3109/1743539 0.2014 .988664

Wang Y, Chen Z, Ba T et al (2013) Susceptibility of young and adult rats to the oral toxicity of titanium dioxide nanoparticles. Small 9(9-10):1742-1752. doi:10.1002/smll.201201185

Wang M, Zheng LN, Wang B et al (2014) Quantitative analysis of gold nanoparticles in single cells by laser ablation inductively coupled plasma-mass spectrometry. Anal Chem 86(20):10252-10256. doi:10.1021/ac502438n

Watkinson AC, Bunge AL, Hadgraft J, Lane ME (2013) Nanoparticles do not penetrate human skin-a theoretical perspective. Pharm Res 30(8):1943-1946. doi:10.1007/s11095-013-1073-9

Westerhout J, van de Steeg E, Grossouw D et al (2014) A new approach to predict human intestinal absorption using porcine intestinal tissue and biorelevant matrices. Eur J Pharm Sci 63:167-177. doi:10.1016/j. ejps.2014.07.003

Whitesides GM (2006) The origins and the future of microfluidics.Nature 442(7101):368-373. doi:10.1038/nature 05058

Wick P, Malek A, Manser P et al (2010) Barrier capacity of human placenta for nanosized materials. Environ Health Perspect118(3):432-436. doi:10.1289/ehp.0901200

Wickham M, Faulks R, Mills C (2009) In vitro digestion methods for assessing the effect of food structure on allergen breakdown. Mol Nutr Food Res 53(8):952-958. doi:10.1002/ mnfr.200800193

Wolff A, Antfolk M, Brodin B, Tenje M (2015) In vitro blood-brain barrier models-an overview of established models and new microfluidic approaches. J Pharm Sci. doi:10.1002/jps.24329

Worth AP, Balls M (2004) The principles of validation and the ECVAM validation process. Altern Lab Anim 32(Suppl 1B):623-629

Worth A, Barroso J, Bremer S et al (2014) Alternative methods for regulatory toxicology-a state of the art review. JRC Sci Policy Rep EUR 26797:1-475

Wu J, Liu W, Xue C et al (2009) Toxicity and penetration of TiO2 nanoparticles in hairless mice and porcine skin after subchronic dermal exposure. Toxicol Lett 191(1):1-8. doi:10.1016/j. toxlet.2009.05.020

Xie Y, Williams NG, Tolic A et al (2012) Aerosolized ZnO nanoparticles induce toxicity in alveolar type II epithelial cells at the air-liquid interface. Toxicol Sci 125(2):450-461. doi:10.1093/ toxsci/kfr251

Yacobi NR, Demaio L, Xie J et al (2008) Polystyrene nanoparticle trafficking across alveolar epithelium. Nanomedicine 4(2):139145. doi:10.1016/j.nano.2008.02.002

Yamashita K, Yoshioka Y, Higashisaka K et al (2011) Silica and titanium dioxide nanoparticles cause pregnancy complications in mice. Nat Nanotechnol 6(5):321-328. doi:10.1038/ nnano.2011.41

Young AM, Allen CE, Audus KL (2003) Efflux transporters of the human placenta. Adv Drug Deliv Rev 55(1):125132

Yu LE, Yung L-YL, Ong C-N et al (2007) Translocation and effects of gold nanoparticles after inhalation exposure in rats. Nanotoxicology 1(3):235-242. doi:10.1080/17435390701763108

Zangenberg NH, Mullertz A, Kristensen HG, Hovgaard L (2001) A dynamic in vitro lipolysis model. II: evaluation of the model. Eur J Pharm Sci 14(3):237-244

Zattoni A, Roda B, Borghi F, Marassi V, Reschiglian P (2014) Flow field-flow fractionation for the analysis of nanoparticles used in drug delivery. J Pharm Biomed Anal 87:53-61. doi:10.1016/j. jpba.2013.08.018

Zhang Z, Kong F, Vardhanabhuti B, Mustapha A, Lin M (2012) Detection of engineered silver nanoparticle contamination in pears. J Agric Food Chem 60(43):10762-10767. doi:10.1021/ jf303423q

Zhu Y, Choe CS, Ahlberg S et al (2015) Penetration of silver nanoparticles into porcine skin ex vivo using fluorescence lifetime imaging microscopy, Raman microscopy, and surface-enhanced Raman scattering microscopy. J Biomed Opt 20(5):051006. doi:10.1117/1.jbo.20.5.051006

Zvyagin AV, Zhao X, Gierden A, Sanchez W, Ross JA, Roberts MS (2008) Imaging of zinc oxide nanoparticle penetration in human skin in vitro and in vivo. J Biomed Opt 13(6):064031. doi:10.1117/1.3041492 


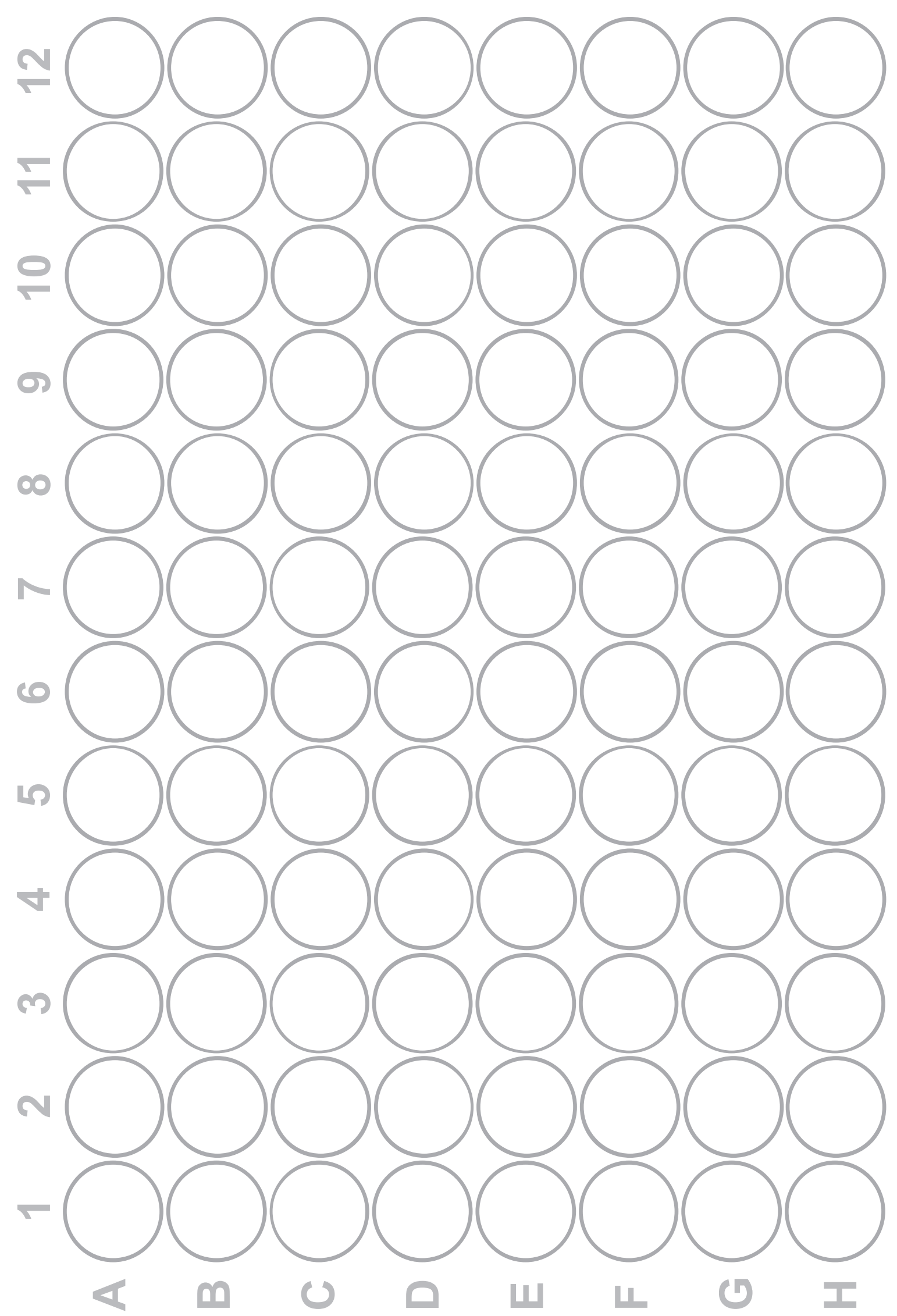




\section{Chapter 3}

\section{Translocation of positively and negatively charged polystyrene nanoparticles in an in vitro placental model}

Samantha K. Kloet Agata P. Walczak Jochem Louisse Hans H.J. van den Berg

Hans Bouwmeester Peter Tromp

Remco G. Fokkink Ivonne M.C.M. Rietjens 


\section{ABSTRACT}

To obtain insight in translocation of nanoparticles across the placental barrier, translocation was studied for one positively and two negatively charged polystyrene nanoparticles (PS-NPs) of similar size in an in vitro model. The model consisted of BeWo b30 cells, derived from a human choriocarcinoma grown on a transwell insert forming a cell layer that separates an apical from a basolateral compartment. PS-NPs were characterized with respect to size, surface charge, morphology and protein corona. Translocation of PS-NPs was not related to PS-NP charge. Two PS-NPs were translocated across the BeWo transwell model to a lower extent than amoxicillin, a model compound known to be translocated over the placental barrier to only a limited extent, whereas one PS-NP showed a slightly higher translocation. Studies on the effect of transporter inhibitors on the translocation of the PS-NPs indicated that their translocation was not mediated by known transporters and mainly dependent on passive diffusion. It is concluded that the BeWo b30 model can be used as an efficient method to get an initial qualitative impression about the capacity of NPs to translocate across the placental barrier and set priorities in further in vivo studies on translocation of NPs to the fetus. 


\section{INTRODUCTION}

Nanoparticles (NPs) are incorporated in a variety of consumer products, such as electronics, clothing, food, cosmetics and medicines. Consequently, there is an increasing potential for workers and consumers to be exposed to various NPs. Due to their small size and relatively large surface area compared to volume, NPs have physico-chemical characteristics different from the bulk material (Auffan et al. 2009). Although these novel characteristics drive the development of numerous applications in many fields of technology and medicine, they may also have unforeseen (adverse) health effects (Oberdorster 2004, Stampfl et al. 2011). It is known that size and surface functionalization are major factors that influence kinetics, including rate of uptake, distribution, and excretion of NPs, and thereby their possible toxicity (Oberdorster et al. 2005, Sager et al. 2009, Park et al. 2011, Bhattacharjee et al. 2013). In order to bypass in vivo toxicity testing of every type of NP, efficient in vitro research methods with prognostic potential would be of great value. Therefore, suitable in vitro methods are needed that can be used to predict the in vivo kinetics of NPs in order to predict in vivo internal exposure. At present, research on transplacental transfer of nanomaterials and their effects on the placenta and the developing fetus is still in its infancy (Saunders 2009, Buerki-Thurnherr et al. 2012, Juch et al. 2013). However, the fetus may be exposed to NPs upon translocation across the placenta, (Menjoge et al. 2011).

The aim of the present study was to obtain insight in translocation of NPs across the placental barrier by studying translocation of positively and negatively charged polystyrene NPs (PSNPs) across an in vitro model. The model consisted of BeWo b30 cells, derived from a human choriocarcinoma, grown on a transwell insert forming a cell layer that separates an apical compartment from a basolateral compartment. When grown on transwell inserts, BeWo b30 cells retain functional adenosine triphosphate binding cassette $(\mathrm{ABC})$ transporters specific to the apical and basolateral membranes, such as P-glycoprotein (Pgp), breast cancer resistance protein (BCRP) or multidrug resistance-associated protein 1 (MRP-1) (Utoguchi et al. 2000, Atkinson et al. 2003, Ceckova et al. 2006, Evseenko et al. 2006, Milane et al. 2009, Mitra et al. 2010, Wick et al. 2010). Furthermore, these cells express placental differentiation markers, such as human chorionic gonadotrophin (HCG) (Takeuchi et al. 1990) and the major cytochrome P450 isoforms (CYP1A1 and 1A2) present in placenta (Avery et al. 2003). In our previous studies the BeWo b30 transwell model was shown to be useful to study placental translocation of drugs and nutrients, and translocation of these compounds across the BeWo b30 layer in the transwell model appeared to correlate with their translocation in an ex vivo human placental model (Li et al. 2013).

In the present study the BeWo b30 transwell model was used to study possible placental translocation of NPs. The model NPs selected for this study were $50 \mathrm{~nm}$ PS-NPs with positive or 
negative charge. PS-NPs are used in various products like food packaging, drug delivery systems, sensors, paints and electronics spacers in LCD displays (Phosphorex, Simon et al. 2008, Arora et al. 2010, Lee et al. 2014, Chiu et al. 2015). PS-NPs were characterized by measuring their size and polydispersity index (PDI) by dynamic light scattering (DLS), their surface potential (as an indication of surface charge) by $\zeta$-potential measurements, their protein corona by SDS-PAGE and their morphology by scanning electron microscopy (SEM).

\section{MATERIALS AND METHODS}

\section{Chemicals}

Fluorescent red PS-NPs were obtained from Magsphere Inc. (Cat nr: AMFR050NM, CAFR050NM). Fluorescent Yellow-Green carboxylated $50 \mathrm{~nm}$ microspheres were obtained from Polysciences (Cat nr. 16661-10). 3-(4,5-Dimethylthiazol-2-yl)-2,5-diphenyltetrazolium bromide (MTT), fluorescein, antipyrine, amoxicillin, $\beta$-mercaptoethanol and Dulbecco's modified Eagle's medium (DMEM) (Cat nr. D5071 and D5671) were purchased from Sigma-Aldrich (Zwijndrecht, the Netherlands). Penicillin-streptomycin, L-glutamine, trypsin-EDTA, fetal calf serum (FCS), phosphate-buffered saline (PBS), and Hank's balanced salt solution (HBSS) were obtained from Invitrogen (Breda, the Netherlands). Laemmli Sample Buffer, Precision Plus Protein Dual Color Standards marker, Bio-Safe Coomassie Stain G-250 and Mini-PROTEAN TGX Gels were obtained from Bio-rad (Veenendaal, the Netherlands).

\section{Cell culture}

The choriocarcinoma BeWo cell line (clone b30) was kindly provided by the Institute of Public Health of the Faculty of Health Sciences (University of Copenhagen, Denmark) with permission from Dr. Alan Schwartz (Washington University, St. Louis, MO) and confirmed to be mycoplasma negative. Cells were cultured at $37^{\circ} \mathrm{C}$ with $5 \% \mathrm{CO}_{2}$ in a humidified atmosphere in DMEM supplemented with $10 \%(\mathrm{v} / \mathrm{v})$ heat inactivated FCS, $1 \%(\mathrm{v} / \mathrm{v})$ penicillin-streptomycin and $1 \%(\mathrm{v} / \mathrm{v})$ L-glutamine. Cells were subcultured every 3 to 4 days when reaching $80 \%$ confluency using trypsin-EDTA after rinsing with PBS.

\section{Particle characterization}

The size (hydrodynamic diameter) of the PS-NPs was determined by dynamic light scattering (DLS) using a Cobolt Samba 300mW DPSS laser at a wavelength of $532 \mathrm{~nm}$ as a light source. Measurements were performed on freshly prepared NP solutions at $10 \mu \mathrm{g} / \mathrm{ml}$ in nanopure water and in cell culture medium containing 10\% FCS. For each sample, twenty measurements were made and the detector intensity data (measured with an ALV/SO SIPD Single Photon Detector with ALV Static and Dynamic Fiber optics) were processed with an ALV5000/60X0 external correlator and ALV-5000/E software (all from ALV-GmbH, Germany). 
Data were analysed with the AfterALV software and mean diameter and polydispersity index (PDI) were determined for each PS-NP sample. Additionally the $\zeta$-potential of the PS-NPs was measured at a concentration of $100 \mu \mathrm{g} / \mathrm{ml}$ using the Malvern Zetasizer 2000 (Malvern Instruments, UK). Three independent measurements, each containing 5 sub-measurements, were used to establish the average $\zeta$-potential and standard deviation (SD) for each sample. Morphology of the PS-NPs was characterised by scanning electron microscopy (SEM). To this end suspensions of the PS-NPs $(20 \mu \mathrm{g} / \mathrm{mL})$ in water were studied using SEM. Of each suspension $20 \mu \mathrm{l}$ was deposited on a nickel-coated polycarbonate filter, which was mounted on an aluminium specimen holder. The droplets were left to dry. Subsequently the filters with PSNPs were coated with a $5 \mathrm{~nm}$ layer of chromium with an Emitech K575X turbo sputter coater. The high resolution field emission gun scanning electron microscope (FEG-SEM) used in this study was a Tescan MIRA-LMH FEG-SEM (Tescan, Brno, Czech Republic). The microscope was operated at an accelerating voltage of $15 \mathrm{kV}$, working distance (WD) of $10 \mathrm{~mm}$ and spot size of $5 \mathrm{~nm}$. The mean diameter \pm SD of 50-500 particles is given.

\section{Protein corona detection}

For the protein corona characterization, methods were adapted from previous similar studies (Lundqvist et al. 2008, Lesniak et al. 2010). Briefly, PS-NPs were suspended in DMEM cell culture medium containing $10 \%$ FCS at a concentration of $1.66 \mathrm{mg} / \mathrm{ml}$ and incubated for 24 hours at $37^{\circ} \mathrm{C}$. The samples were centrifuged $40 \mathrm{~min}$ at $18000 \mathrm{rcf}$ at $15^{\circ} \mathrm{C}$ to pellet the NP-protein complexes. The pellet was resuspended in $1 \mathrm{ml} \mathrm{PBS}$, transferred to a new tube and centrifuged again. This washing procedure was repeated three times to eliminate non-firmly bound proteins, using a new tube in every wash step to avoid artifacts from proteins bound to the tube wall. Finally, after the last centrifugation, the supernatant was removed and the pellets were resuspended carefully with $70 \mu \mathrm{l}$ of a 1:1 (v/v) mixture of PBS and 2x Laemmli Sample Buffer containing 5\% $\beta$-mercaptoethanol. Next, the samples were boiled for $5 \mathrm{~min}$ at $98^{\circ} \mathrm{C}$ to denature the proteins and unbind the proteins from the PS-NPs and then cooled down immediately on ice. Samples were again centrifuged $40 \mathrm{~min}$ at $18000 \mathrm{rcf}$ at $15^{\circ} \mathrm{C}$ to separate the PS-NPs from proteins desorbed from their surface. Of the supernatant containing the desorbed proteins $20 \mu \mathrm{l}$ was loaded on the gel. In this way the amount of protein loaded in each lane was normalized for the weight of the different PS-NPs in the sample. A second gel was loaded with samples that were normalized for amount of protein in each lane. As we could not measure the protein content because of interference of $\beta$-mercaptoethanol which was present and required in all samples, an indirect measurement of total protein present in the samples was used. To this end the acquired gel image of the first gel was digitally analysed for the pixel intensity of each whole lane. We thus obtained an estimate of the amount of protein loaded in each sample. The pixel intensity of all lanes was expressed relative to the pixel intensity of the lane with the lowest pixel intensity. This resulted in a factor by which the 
samples should be diluted in order to load the same amount of protein in all lanes of the second gel. One-dimensional polyacrylamide gel electrophoresis was performed using standard methods on the Bio-Rad Mini-Protean system, at $90 \mathrm{~V}$ for about $80 \mathrm{~min}$, until the proteins neared the end of the gel (Mini-PROTEAN TGX Gel, 12\% polyacrylamide $1 \mathrm{~mm}$ ). A marker sample was included on each gel. The gels were washed in $40 \%$ methanol in water containing $10 \%$ acetic acid for $15 \mathrm{~min}$ and subsequently stained with Bio-Safe Coomassie Stain G-250 for 1.5 hours. Afterwards, the gels were washed extensively and stored overnight in distilled water. Gels were scanned using the Odyssey scanner (Li-cor ISO 9001) and analysed by further quantification of the band intensity of the lanes with the Odyssey software (Li-cor Biosciences). Experiments were repeated three times.

\section{MTT assay}

BeWo b30 cells were seeded at $1 \times 10^{4}$ cells/well in $100 \mu \mathrm{l}$ culture medium in 96-well plates and incubated at $37^{\circ} \mathrm{C}$ and $5 \% \mathrm{CO}_{2}$ for 24 hours, followed by exposure to $100 \mu \mathrm{l}$ of PS-NP solutions with concentrations ranging from $0 \mu \mathrm{g} / \mathrm{ml}$ to $100 \mu \mathrm{g} / \mathrm{ml}$. After 24 hours $10 \mu \mathrm{l}$ of MTT solution in PBS $(5 \mathrm{mg} / \mathrm{ml})$ was added to the medium to reach a final concentration of $0.5 \mathrm{mg} / \mathrm{ml}$ MTT. After 1.5 hours incubation, the MTT solution was removed and $100 \mu \mathrm{l} /$ well pure DMSO were added to lyse the cells and to dissolve the formazan crystals. The plates were shaken for 10 minutes protected from light and subsequently absorbance was measured at 562 and 620 (background) nm using a SpectraMax M2 Microplate Reader (Molecular Devices LLC, Sunnyvale, CA USA). For each PS-NP concentration cellular MTT reduction to formazan is expressed as percentage of the corresponding DMEM control reading $(0 \mu \mathrm{g} / \mathrm{ml})$. The absence of possible interference of particle fluorescence with the absorbance readings was confirmed by reading the absorbance of wells with different concentrations of NPs after adding MTT and this indicated there was no interference of the particles with the absorbance readings.

\section{Translocation studies}

Originally transwell membranes are coated with placental collagen before seeding the BeWo b30 cells (Bode et al. 2006). However, previous studies showed that this collagen coating inhibits NP transfer across the permeable membrane (Cartwright et al. 2012). Therefore, no collagen coating was used in the translocation studies. This will present a model presenting the highest possible translocation and thus preventing underestimation of the translocation. BeWo b30 cells were seeded at a density of 100.000 cells $/ \mathrm{cm}^{2}$ on 12 -well transwell inserts containing a polycarbonate membrane $\left(0.4 \mu \mathrm{m}\right.$ pore size and a surface area of $\left.1.12 \mathrm{~cm}^{2}\right)$ (Corning, NY, product number 3401) with daily refreshment of the medium as described by (Poulsen et al. 2009). At day 6 post-seeding the BeWo b30 cell layers were washed twice with prewarmed $\left(37^{\circ} \mathrm{C}\right) \mathrm{HBSS}$ without phenol red after which transepithelial electrical resis- 
tance (TEER) measurements were performed just before exposure using a Millicell ERS-2 Volt-Ohm Meter (Millipore, USA). Cell layers showing TEER values $>160 \mathrm{ohm} \mathrm{cm}^{2}$ were chosen for translocation experiments, as these were values reported by others indicating a intact barrier (Liu et al. 1997, Li et al. 2013). After TEER measurement, HBSS was removed and replaced by exposure medium.

Translocation of the two reference compounds amoxicillin and antipyrine (both at final concentration $100 \mu \mathrm{M}$ ) was assessed by adding apically $0.5 \mathrm{ml}$ of the test compound dissolved in HBSS and basolaterally $1.5 \mathrm{ml}$ of HBSS. In order to ensure the quality of the BeWo model, we always include both antipyrine and amoxicillin in our transport studies, using the circumstances (HBSS without protein) as used in our validation study (Li et al., 2013). In that study, (Li et al., 2013) showed that the transport of antipyrine and amoxicillin is not affected by the presence of proteins in the medium. At selected time points a sample of $0.2 \mathrm{ml}$ was taken from the basolateral compartment and replaced by an equal volume of HBSS. At the end of each experiment $0.2 \mathrm{ml}$ was also taken from the apical compartment. Subsequently cells were washed three times with HBSS without phenol red, TEER values were determined to check for an intact barrier. If the TEER value after the translocation study was not lower than before the translocation study, the experiment was considered valid, and the membrane with cells was dissected and transferred to an Eppendorf tube containing $250 \mu \mathrm{l} 65 \%$ (v/v) methanol to determine the amount of compound accumulated in the cells using HPLC.

Translocation of PS-NPs in time was tested by adding $0.5 \mathrm{ml}$ of the freshly prepared vortexed PS-NP dilutions in phenol red free DMEM supplemented with 10\% FCS to the apical compartment. At selected time points, samples of $100 \mu \mathrm{l}$ were taken from the apical and basolateral compartments and at the end of the incubation the membranes with BeWo b30 cell layers were washed three times with $\operatorname{HBSS}\left(37^{\circ} \mathrm{C}\right)$ without phenol red and TEER values were measured to check cell layer integrity. Then membranes were dissected and transferred to an Eppendorf tube containing $400 \mu \mathrm{l}$ cold nanopure water. Samples were sonicated in order to lyse the cells and samples of $100 \mu \mathrm{l}$ were taken and added to a 96 wells plate to measure fluorescence for PS-NP quantification. PS-NP quantification was done by fluorescence measurements using a calibration curve (Magsphere PS-NPs: $\lambda_{\mathrm{ex}}=540, \lambda_{\mathrm{em}}=630$, Polysciences PSNPs $\lambda_{\mathrm{ex}}=485, \lambda_{\mathrm{em}}=530$ ) using a SpectraMax M2 Microplate Reader (Molecular Devices LLC, Sunnyvale, CA USA).

The role of specific transporters in the translocation of PS-NPs was tested by performing the translocation experiments in the presence or absence of the transporter inhibitors verapamil (inhibitor of PgP), KO143 (inhibitor of human breast cancer resistance protein; BCRP), MK571 (inhibitor of multidrug resistance-associated protein 1; MRP-1), chlorpromazine (inhibitor of caveolin-mediated endocytosis) or methyl- $\beta$-cyclodextrin $(\mathrm{M} \beta \mathrm{CD}$ ) (inhibitor of clathrin-mediated endocytosis). Table S1 presents an overview of the different transporter 
inhibitors used, their transporter specificity, their concentrations generally used in literature to study transporter inhibition and relevant references. All inhibitors were diluted from a 250 times concentrated stock solution in DMSO in cell culture medium, except for M $\beta C D$ which was diluted from a 250 times concentrated stock solution in PBS. Transporter inhibitors were also added to the basolateral compartment containing $1.5 \mathrm{ml}$ culture medium, using the same concentrations as applied in the apical compartment. Subsequently, the plate was incubated in a humidified atmosphere with $5 \% \mathrm{CO}_{2}$ at $37^{\circ} \mathrm{C}$. After 24 hours, samples of $100 \mu$ l were taken and PS-NPs were quantified by fluorescence measurements in the same way as previously described.

\section{High performance liquid chromatography analysis}

To determine the transport rate of amoxicillin and antipyrine, the collected samples were quantified using High Performance Liquid Chromatography (HPLC) as was described by (Li et al. 2013).

\section{Data analysis}

The apparent permeability coefficients (Papp; $\mathrm{cm} / \mathrm{s}$ ) were calculated according to:

$$
\text { Papp }=(\mathrm{dQ} / \mathrm{dt}) /\left(\mathrm{A} \mathrm{x} \mathrm{C}_{0}\right)
$$

In which $\mathrm{dQ} / \mathrm{dt}$ is the transport velocity $[\mathrm{nmol} / \mathrm{s}$ or $\mu \mathrm{g} / \mathrm{s}]$ in the initial phase when appearance in the basolateral chamber is linear in time, A - the surface area of the membrane insert $\left[\mathrm{cm}^{2}\right]$ and $\mathrm{C}_{0}$ - the initial concentration of the compound in the donor chamber $(\mathrm{nmol} / \mathrm{ml} \mathrm{or} \mu \mathrm{g} / \mathrm{ml})$ (Artursson et al. 1991).

\section{Statistical analysis}

Each experiment was repeated at least three or four times and the results are shown as mean \pm standard deviation (SD). Statistical analyses were performed using GraphPad Prism ${ }^{\bullet}$ version 5, using a one-way analysis of variance ANOVA test and post hoc Tukey test to determine significant differences between the groups. Groups were considered significantly different when $\mathrm{p}<0.05$.

\section{RESULTS}

\section{Physico-chemical characterization of the PS-NPs}

This study included three types of PS-NPs, one $50 \mathrm{~nm}$ positively charged PS-NP and two 50 $\mathrm{nm}$ negatively charged PS-NPs. The two $50 \mathrm{~nm}$ negatively charged PS-NPs were included because previous studies of our group revealed that these are translocated to a different extent across a Caco-2 monolayer (Walczak et al. 2014). Several physico-chemical properties of the nanoparticles were characterized. 
Table 1. DLS data of $50 \mathrm{~nm}$ PS-NPs with positive or negative charge in nanopure water and in medium with $10 \%$ FCS.

\begin{tabular}{llll}
\hline Nanoparticle & Medium & Mean size in nm (SD) & Polydispersity Index (PDI) \\
\hline $\begin{array}{l}50 \text { nm positively charged } \\
\text { PS-NP (Magsphere) }\end{array}$ & Nanopure water & $56.5(3.02)$ & 0.09 \\
& DMEM+FCS & $82.7(30.91)$ & 0.49 \\
50 nm negatively charged & Nanopure water & $50.4(1.74)$ & 0.19 \\
PS-NP (Magsphere) & DMEM+FCS & $69.0(10.1)$ & 0.49 \\
50 nm negatively charged & Nanopure water & $52.4(0.1)$ & 0.21 \\
PS-NP (Polysciences) & DMEM+FCS & $57.6(11.7)$ & 0.50 \\
\hline
\end{tabular}
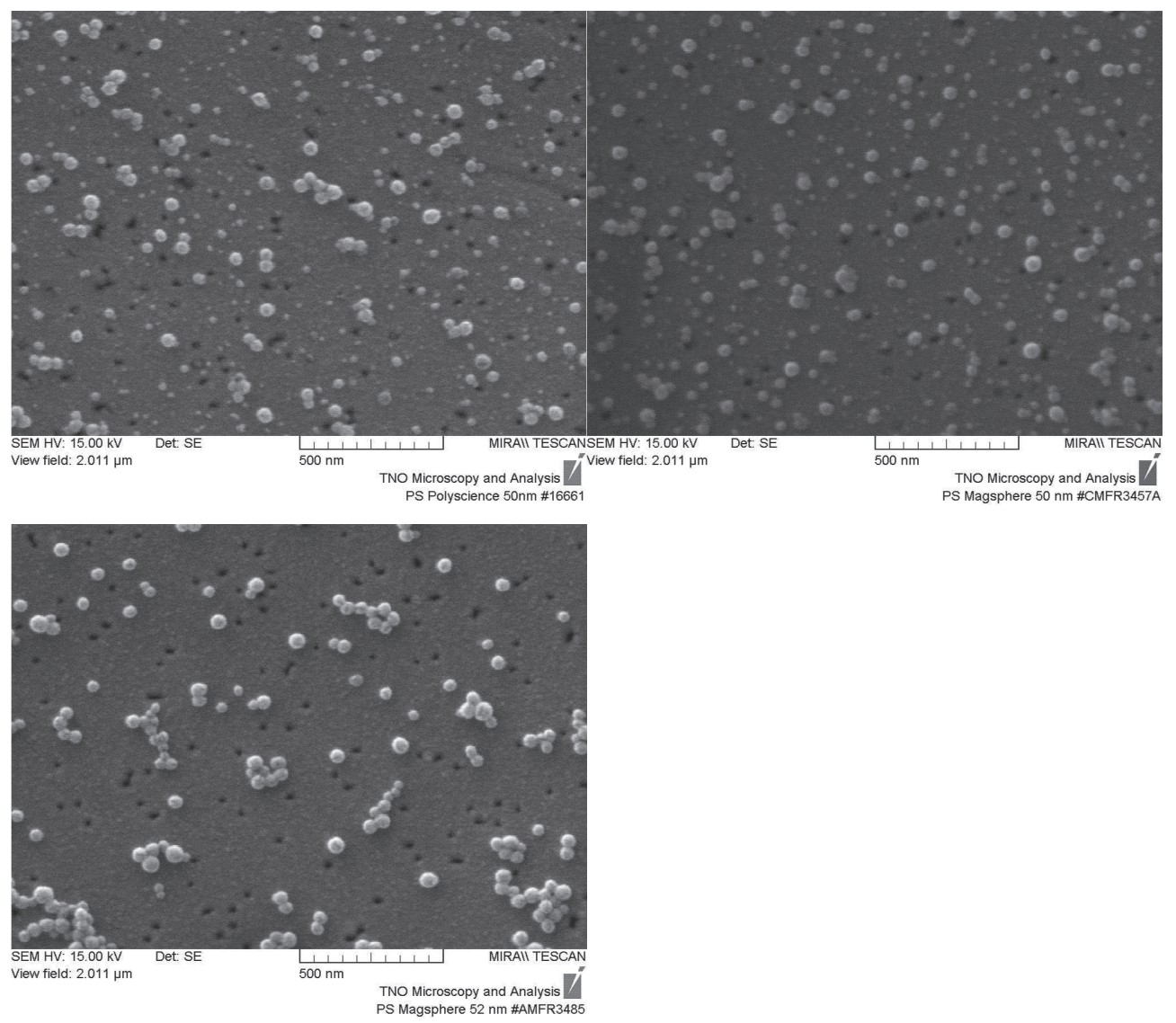

Figure 1. SEM pictures of A) $50 \mathrm{~nm}$ negatively charged PS-NP (Polysciences), B) $50 \mathrm{~nm}$ negatively charged PS-NP (Magsphere) and C) $50 \mathrm{~nm}$ positively charged PS-NP (Magsphere). 
SEM pictures of the PS-NPs (Figure 1) confirm the spherical shape of the particles and their diameter of around $50 \mathrm{~nm}$, although smaller particles can be observed in all samples. Automatic diameter measurements of these desiccated PS-NPs show that their sizes range between $15-75 \mathrm{~nm}$ with a mean value at $31.6 \mathrm{~nm}, 35 \mathrm{~nm}$ and $50.6 \mathrm{~nm}$ for the negatively charged (Polysciences), negatively charged (Magsphere) and positively charged (Magsphere) PS-NPs respectively (Table S2).

The hydrodynamic diameters of the hydrated NPs were measured by dynamic light scattering (DLS) in nanopure water and in the culture medium containing 10\% FCS as used for the translocation experiments (Table 1). The measured sizes of the PS-NPs in nanopure water were close to the sizes reported by the manufacturer. Upon addition of the positively charged PS-NPs to DMEM with 10\% FCS, their size increased 1.5-fold. The measured sizes of the negatively charged PS-NPs (Magsphere) and negatively charged PS-NPs (Polysciences) in DMEM with $10 \%$ FCS increased slightly by 1.4 and 1.1 fold, respectively (Table 1 ). It is of interest to note that DLS analysis of medium with 10\% FCS alone revealed that proteins from the FCS appear to form particles of comparable sizes as the NPs (Figure S1). Formation of protein complexes in the nm range have been reported before (Liu et al. 2013). Thus, DLS measurements of NPs in medium containing 10\% FCS may be hampered by the protein aggregates of comparable size.

The surface charges of the positively and negatively charged PS-NPs were measured in nanopure water and in DMEM with 10\% FCS by determining the zeta potential (Table 2). Positively charged PS-NPs had a zeta potential of $+51.0 \mathrm{mV}$ in nanopure water. Negatively charged PS-NPs of Magsphere and Polysciences have a zeta potential of $-55.5 \mathrm{mV}$ and $-58.3 \mathrm{mV}$ respectively in nanopure water. In medium with $10 \%$ FCS all NPs get a similar slightly negative

Table 2. Zeta potential of $50 \mathrm{~nm}$ PS-NPs with positive or negative charge in nanopure water and in medium with $10 \%$ FCS, and zeta potential measured with medium with $10 \%$ FCS.

\begin{tabular}{llll}
\hline Nanoparticle & Medium & Mean Zeta $(\mathbf{m V})$ & SD \\
\hline 50 nm positively charged PS-NP (Magsphere) & Nanopure water & 51 & 4.5 \\
& DMEM+FCS & -12.0 & 1.2 \\
50 nm negatively charged PS-NP (Magsphere) & Nanopure water & -55.5 & 6.7 \\
& DMEM+FCS & -11.1 & 0.9 \\
50 nm negatively charged PS-NP (Polysciences) & Nanopure water & -58.3 & 2.7 \\
& DMEM+FCS & -10.9 & 0.2 \\
None & DMEM+FCS & -9.16 & 1.3 \\
\hline
\end{tabular}


charge. This may be explained by the fact that the majority of particles present in these samples are protein aggregates from the 10\% FCS so that the zeta potential may reflect the overall charge of the protein aggregates rather than those of the PS-NPs (Table 2). Alternatively the similar charges in the presence of FCS may be ascribed to the protein corona dominating the overall charge.

\section{Protein corona detection}

Figure 2A shows a representative picture of the SDS-PAGE gel with proteins desorbed from the different PS-NPs after their incubation in medium with $10 \%$ FCS. Each lane contains the amount of protein obtained from the same amount of PS-NP and thus, given the similar size of the PS-NPs, presents the amount of protein desorbed from a presumably similar surface area of the PS-NPs. Proteins desorbed from the protein corona of NPs are present in lanes 2-4. Figure $2 \mathrm{~B}$ shows a protein gel prepared to contain the same amount of protein in each lane. The band intensities of the lanes containing NPs were further quantified using the Odyssey software and graphs thus obtained are also presented in Figure 2. These results reveal differences in the amount but not the nature of the proteins in the corona of the three different PS-NPs (Figure 2A+B lanes 2-4).

From the $50 \mathrm{~nm}$ positively charged PS-NPs (lane 3) only a small amount of protein corona was obtained. The control (lane 5) shows the DMEM sample without nanoparticles which was treated by the same experimental procedure as NP samples. Hardly any protein is detected in this control, which corroborates that the proteins which are detected in the other samples are coming from the protein corona of the PS-NPs. Lane 6 shows the proteins present in the medium containing 10\% FCS and comparison of this pattern to that of the PS-NPs reveals that not all medium proteins contribute to the protein corona to the same relative extent at which they are present in the medium, indicating at least some preferential binding of proteins to the NPs.

\section{Cell viability studies}

Before testing the transplacental translocation of the PS-NPs, the concentration of the PS-NPs to be tested in the BeWo b30 transwell translocation studies were chosen based on results from cell viability studies. The cell viability of BeWo b30 cells upon 24 hours exposure to increasing PS-NP concentrations was determined using the MTT assay. Only cells exposed to the positively charged PS-NPs showed a decrease in MTT reduction, an effect that becomes statistically significant at concentrations of $20 \mu \mathrm{g} / \mathrm{ml}$ and higher (Figure 3), whereas no effect was found upon exposure to the negatively charged PS-NPs. Based on these results the concentration of PS-NPs to be tested in the translocation studies was chosen at $10 \mu \mathrm{g} / \mathrm{ml}$. 

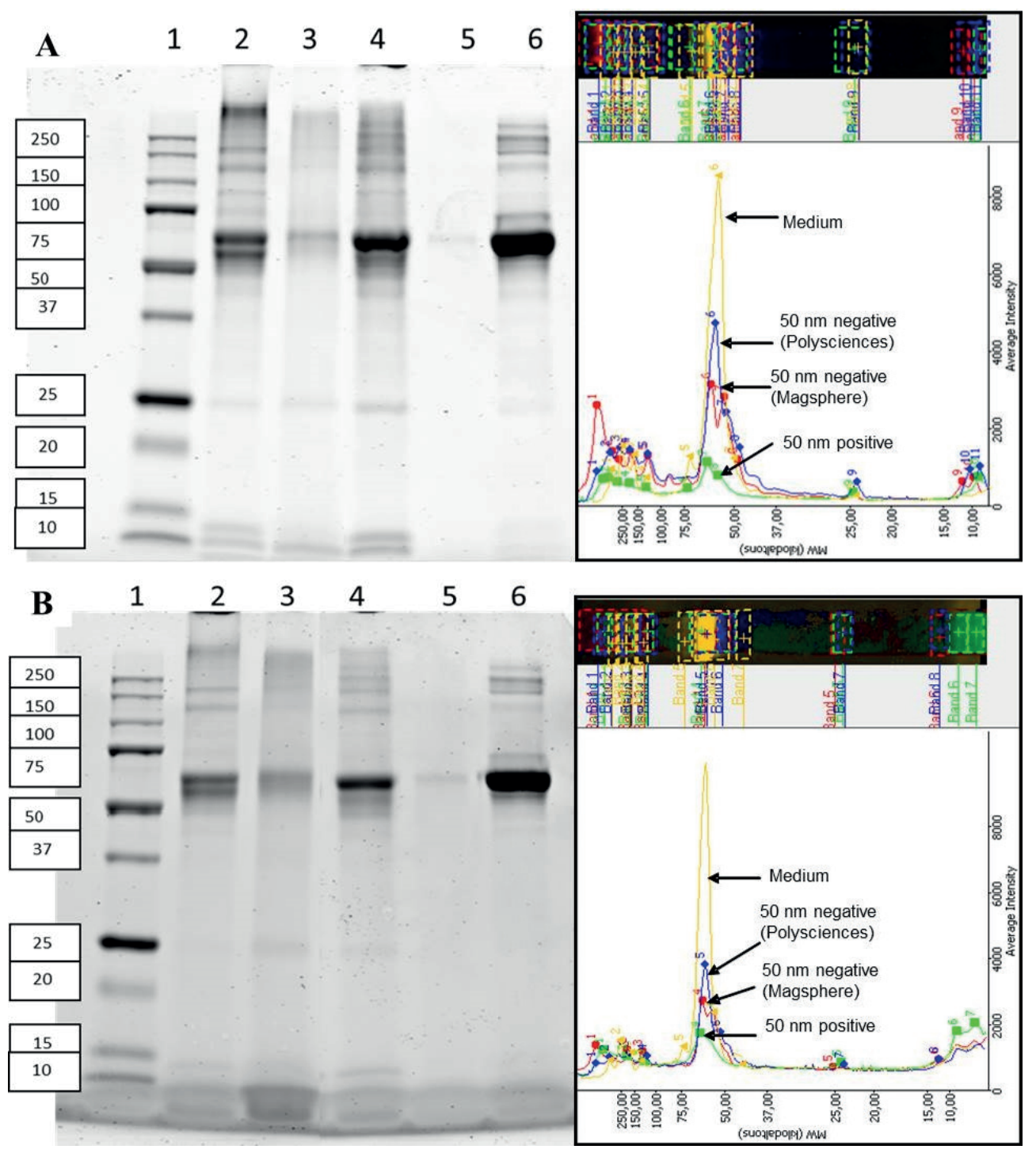

Figure 2. SDS-PAGE gel showing the desorbed protein corona of different PS-NPs with the lanes containing: in gel A) the amount of protein corresponding to the same surface area of the PS-NPs and those in gel B) a similar amount of total protein. Lanes: 1) marker; 2) $50 \mathrm{~nm}$ negatively charged (Magsphere); 3) $50 \mathrm{~nm}$ positively charged (Magsphere); 4) $50 \mathrm{~nm}$ negatively charged (Polysciences); 5) Control: medium sample without NPs which followed the same experimental procedure as the NP-samples; 6) Medium. The right part of the figure shows the banding pattern of lanes 2-4 and 6. 


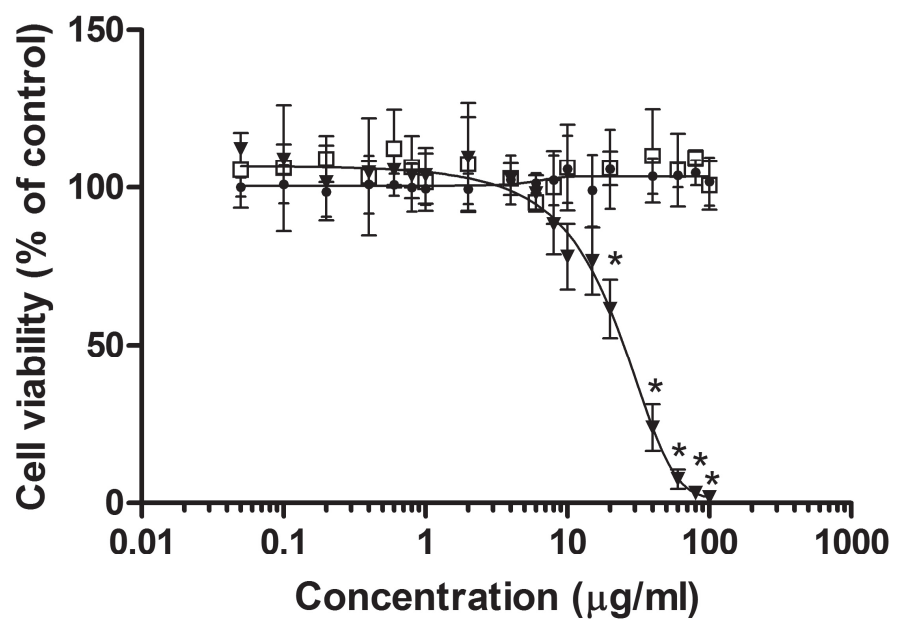

Figure 3. Effects of three different PS-NPs on cell viability of BeWo b30 cells as determined with the MTT assay. BeWo b30 cells were exposed for 24 hours to increasing concentrations of 50 nm positively charged PS-NP (Magsphere) (open triangle) or $50 \mathrm{~nm}$ negatively charged PS-NP (Magsphere) (closed circle) or $50 \mathrm{~nm}$ negatively charged PS-NP (Polysciences) (open square) PS-NPs. Data are expressed as percentage of the negative control and presented as the mean \pm SD of $n=3$ independent biological replicates. The ${ }^{\star}$ indicates $p<0.05$ for a significant difference with the other two types of PS-NPs $(n=3)$.

\section{Placental translocation of model compounds}

In order to calibrate the BeWo b30 model system and provide points for comparison the translocation of two model compounds antipyrine and amoxicillin was determined. These compounds are known to be translocated across the placenta and also across the BeWo cell layer in vitro at respectively high or low rate (Akbaraly et al. 1985, Li et al. 2013). Table 3 shows the apparent permeability coefficients for antipyrine and amoxicillin amounting to 53 $\mathrm{x} 10^{-6} \mathrm{~cm} / \mathrm{s}$ for antipyrine and $5.1 \times 10^{-6} \mathrm{~cm} / \mathrm{s}$ for amoxicillin indicating that the translocation of these two model compounds varies by one order of magnitude. Previously it was reported that for a series of model compounds including antipyrine and amoxicillin a good correlation $\left(\mathrm{r}^{2}=0.95\right)$ exists between their relative transport in the BeWo b30 transwell model and their transport in an ex vivo placental perfusion model (Li et al. 2013) and that also in the ex vivo placental transport model antipyrine and amoxicillin showed a difference in transport rate of about one order of magnitude (Akbaraly et al. 1985). 
Table 3. Apparent permeability (Papp) coefficients across BeWo b30 cell barrier of the reference compounds antipyrine and amoxicillin, and the PS-NPs. Data presented are the mean $\pm S D$ of $n=3$ independent biological replicates.

\begin{tabular}{ll}
\hline & Mean Papp value \pm SD $(\mathrm{cm} / \mathrm{s})$ \\
\hline Antipyrine & $53 \times 10^{-6} \pm 5.6 \times 10^{-6}$ \\
Amoxicillin & $5.1 \times 10^{-6} \pm 0.52 \times 10^{-6}$ \\
$50 \mathrm{~nm}$ positively charged PS-NP (Magsphere) & $0.3 \times 10^{-6} \pm 0.05 \times 10^{-6}$ \\
$50 \mathrm{~nm}$ negatively charged PS-NP (Magsphere) & $* \mathrm{ND}$ \\
$50 \mathrm{~nm}$ negatively charged PS-NP (Polysciences) & $13 \times 10^{-6} \pm 1.1 \times 10^{-6}$ \\
\hline
\end{tabular}

${ }^{*} \mathrm{ND}$ : not detected, so no Papp coefficient derived.

\section{Placental translocation of PS-NPs}

The time-dependent translocation of the three PS-NPs to the basolateral compartment was determined using an apical concentration of $10 \mu \mathrm{g} / \mathrm{ml}$ PS-NP (Figure 4). Upon 24 hours incubation the negatively charged PS-NPs from Magsphere were not detected in the basolateral compartment. For the other two PS-NPs a time dependent increase in the basolateral compartment was observed (Figure 4). The apparent permeability coefficient of the positively charged PS-NPs from Magsphere was the lowest (Papp value of $0.3 \times 10^{-6} \mathrm{~cm} / \mathrm{s}$ ), followed by that of the negatively charged PS-NPs from Polysciences (Papp value of $13 \times 10^{-6} \mathrm{~cm} / \mathrm{s}$ ). In the absence of cells, PS-NP translocation across the insert was much higher which corroborates that the BeWo b30 cell layer acts as a barrier.

Comparison of the apparent permeability coefficients of the PS-NPs that were translocated with that of the reference compound amoxicillin, known to be translocated over the placental barrier to only a limited extent, reveals that translocation of the positively charged PS-NPs of Magsphere is 17 fold lower than that of amoxicillin, whereas the translocation of the negatively charged PS-NPs from Polysciences was 2.5 fold higher than that of amoxicillin (Table 3).

\section{Compartmental distribution of PS-NPs in the presence of transporter inhibitors}

To investigate the possible role of specific transport mechanisms in the translocation of the PS-NPs across BeWo b30 cell layers, the distribution of the NPs over the three compartments (apical, basolateral and intracellular) was assessed in the presence or absence of inhibitors of endocytosis or of specific ABC transporters. Results obtained are presented in figure 5. For the positively charged PS-NPs, the presence of MK571, an inhibitor of MRP-1, slightly but significantly $(\mathrm{p}<0.01)$ decreased the amount of PS-NPs in the apical compartment from 71.5 to $67.8 \%$ and significantly increased $(\mathrm{p}<0.01)$ the amount of PS-NPs in the basolateral 


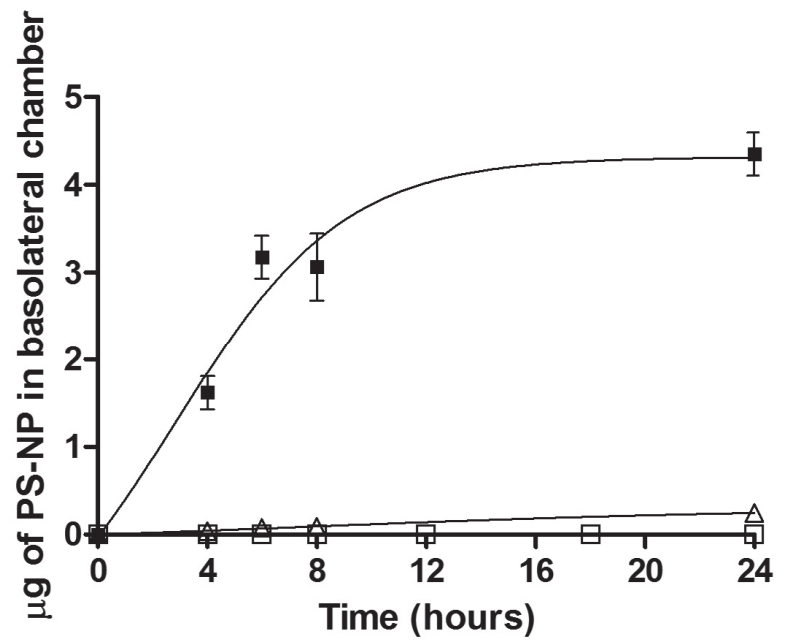

Figure 4. Time dependent translocation across the BeWo b30 cell layer of $50 \mathrm{~nm}$ positively charged PSNPs of Magsphere (open triangle), $50 \mathrm{~nm}$ negatively charged PS-NPs of Magsphere (open square) and $50 \mathrm{~nm}$ negatively charged PS-NPs of Polysciences (closed square) with starting concentration of $10 \mu \mathrm{g} /$ ml. Papp values (Table 3) were calculated from the linear part of the curve. Results presented are the mean $\pm \mathrm{SD}$ of $\mathrm{n}=3$ independent biological replicates.

compartment from 7.5 to $11.5 \%$ (figure $5 \mathrm{~A}$ ), indicating a 1.5 fold increased translocation across the BeWo b30 cell layer. TEER measurements performed after the distribution studies showed that the barrier integrity of the BeWo b30 cell layer was not affected, indicating that the increased translocation did not result from a compromised cell layer. Co-exposure to the positively charged PS-NPs and the BCRP- or PgP-inhibitors KO143 and verapamil, respectively, did not show an effect on the distribution of these PS-NPs (figure 5A). For the negatively charged $50 \mathrm{~nm}$ PS-NPs from Polysciences, none of the inhibitors showed a significant effect on the distribution of these particles in the BeWo transwell model (Figures 5B) and the lack of intracellular signal indicates no intracellular localization. In addition, the clathrin and caveolin inhibitors ( $5 \mu \mathrm{M}$ chlorpromazine or $1 \mu \mathrm{M} \mathrm{M} \beta \mathrm{CD}$, respectively) used to inhibit clathrin- and caveolin- mediated endocytosis, did not show an effect on translocation or distribution of any of the PS-NPs. 

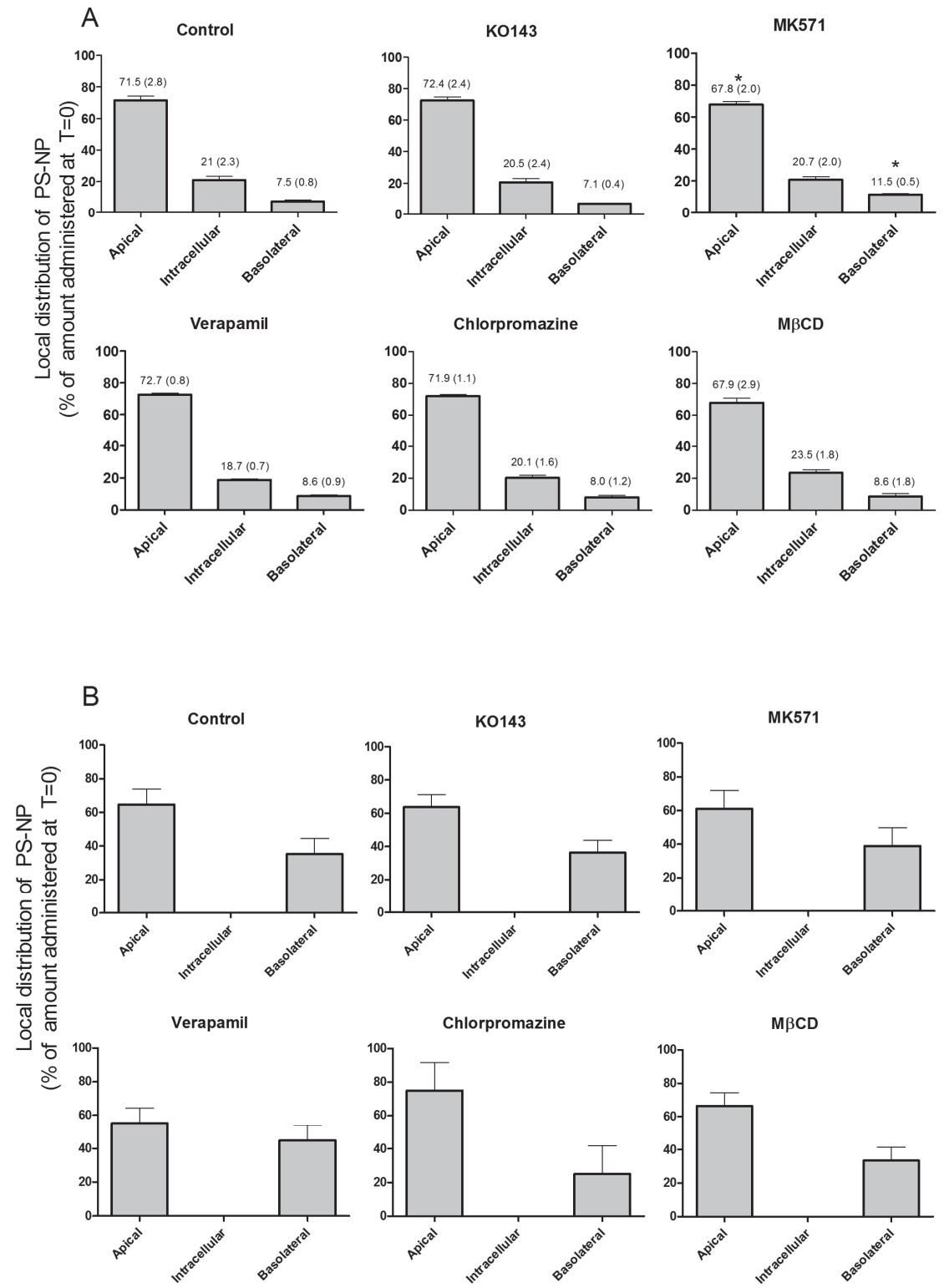

Figure 5. Effect of specific transporter inhibitors (Table S1) on the distribution of A) 50 nm positively charged PS-NPs of Magsphere; B) $50 \mathrm{~nm}$ negatively charged PS-NP of Polysciences in the BeWo transwell model, 24 hours after adding $0.5 \mathrm{ml}$ of a $10 \mu \mathrm{g} / \mathrm{ml}$ PS-NP solution to the apical compartment. Results presented are the mean $\pm \mathrm{SD}$ of $\mathrm{n}=4$ independent biological replicates. The ${ }^{*}$ indicates a difference $(\mathrm{p}<0.01)$ compared to the situation without inhibitors. 


\section{DISCUSSION}

To obtain insight in passage of NPs across the placental barrier, translocation of one positively and two different types of negatively charged PS-NPs across an in vitro model of the placental barrier was studied. The two negatively charged PS-NPs were included because previous studies within our group revealed they were translocated to a different extent across a Caco-2 monolayer, a model for intestinal translocation (Walczak et al. 2014). The model in the present study consisted of cells of the BeWo cell line (clone b30) grown on a transwell insert, and forming a cell layer that separates an apical compartment from a fetal compartment.

The characterization of the PS-NPs was done by measuring the size and polydispersity index (PDI) by dynamic light scattering (DLS), the $\zeta$-potential (as an indication of surface charge) by $\zeta$-potential measurements, the protein corona by SDS-PAGE and shape and the number-based size distribution by scanning electron microscopy (SEM). The results obtained reveal that in the presence of $10 \%$ FCS in cell culture medium, measurement of $\zeta$-potential may be hampered by the presence of free protein particles as the $\zeta$-potential of medium without or with PS-NPs present, resulted in the same negative $\zeta$-potential. Alternatively the similar charges of the differently charged NPs in the presence of FCS may be ascribed to their protein corona dominating the underlying PS-NP's charge. SDS-PAGE analysis of this protein corona revealed that there were differences in mainly the amount but not in the nature of the proteins in the corona of the differently charged PS-NPs. The proteins desorbed from the PS-NPs were to a large extent a reflection of what was detected in the medium containing $10 \%$ FCS. This result was somewhat different from a previous study that reported that surface properties of PS-NPs significantly affected the composition of the protein corona (Lundqvist et al. 2008). In this latter study using different commercially available PS-NPs and mass spectrometry instead of SDS-PAGE, a fraction (about 35\%) of the proteins in the corona were specifically detected on the positively charged PS-NPs or on the negatively charged PS-NPs, the others being similar.

In the present study it appeared that in spite of similar size (SEM and DLS), $\zeta$-potential and type of proteins in the protein corona, the differently charged NPs displayed a remarkable difference in cytotoxicity, with only the PS-NPs with an original positive charge inducing cytotoxicity. The relatively higher cytotoxicity of positively as compared to negatively charged NPs was reported before for tri-block copolymer NPs (Bhattacharjee et al. 2012) but also for other type of NPs like gold NPs (Liu et al. 2013) and silicon NPs (Bhattacharjee et al. 2013). Thus for interaction with the cells, the original difference in charges or surface chemistry of the particles as prepared and measured in water, is apparently still of influence. The higher cytotoxicity of positive NPs might be due to a stronger interaction with cells compared with negative NPs, because of the electrostatic attraction towards the negatively charged cell membrane (Kim et al. 2009). 
Translocation of the different PS-NPs across the BeWo cell layer was not found to be related to PS-NP charge. A remarkable difference in translocation was found between the two $50 \mathrm{~nm}$ negatively charged PS-NPs from different manufacturers. For the PS-NPs from Polysciences, the apparent permeability coefficient was slightly higher than that of amoxicillin, with a Papp value of $13 \times 10^{-6} \mathrm{~cm} / \mathrm{s}$ (table 3 ). When testing the negatively charged PS-NPs from Magsphere, no PS-NPs could be detected in the basolateral compartment upon adding a concentration of $10 \mu \mathrm{g} / \mathrm{ml}$ in the apical compartment and 24 hours incubation. A 30-fold difference was found in translocation of the same types of negatively charged PS-NPs across a Caco-2 monolayer in a similar transwell model system (Walczak et al. 2014). Since none of the characterized parameters, including size, $\zeta$-potential and protein corona revealed remarkable differences between the two negatively charged NPs, the difference may originate from the chemical groups on the surface of the NPs generating the negative charge. Although both PS-NPs are reported by the respective manufacturers to contain carboxylic groups from vinylic co-monomers, including acrylic acid, methacrylic acid and itaconic acid, further details on the chemical characteristics of the NP surface remained unknown. In this respect it is of interest that variation in steric shielding of the charge on a surface moiety due to subtle differences in the chemical structure of the charge bearing moiety may affect the cellular uptake and toxicity of fluorescent tri-block copolymer nanoparticles (Bhattacharjee et al. 2011). In this previous study it was demonstrated that three different positively charged tri-block copolymer nanoparticles showed different cellular uptake and toxicity in spite of a similar overall size and $\zeta$-potential, and that only a different shielding of the charge due to subtle differences in the charge generating surface moiety influenced the cellular uptake and toxicity (Bhattacharjee et al. 2011). This factor may explain the different translocation rates of the two negatively charged $50 \mathrm{~nm}$ PS-NPs in this study, given the different carboxylic groups that have been used in the production of these different PS-NPs.

The translocation studies presented in this study revealed that the PS-NPs were translocated across the BeWo b30 transwell model to only a limited extent. We compared these results to reports in the literature studying the translocation of NPs across the placental barrier. A few studies also applied the BeWo b30 transwell model studying translocation of PEGylated gold NPs of 10-30 nm (Myllynen et al. 2008), iron oxide and silica NPs of 23-38 nm (Correia Carreira et al. 2013), dexamethasone loaded PLGA NPs of 140-289 nm (Ali et al. 2013), rhodamine labelled silica NPs of 25 and $50 \mathrm{~nm}$ (Sonnegaard Poulsen et al. 2013) and polystyrene NPs of 50 and $100 \mathrm{~nm}$ (Cartwright et al. 2012). These studies generally report that the NPs are translocated across the BeWo cell layer after a few hours of incubation to the basolateral compartment albeit in relatively small amounts. The results of the present study are in line with these observations, and suggest that translocation of NPs across the in vitro placental barrier cannot be excluded. Literature data obtained in ex vivo human placental 
perfusion models also report the potential of NPs to cross the placental barrier (Wick et al. 2010, Menjoge et al. 2011, Grafmuller et al. 2013, Sonnegaard Poulsen et al. 2013) and are in line with this conclusion.

In order to investigate the role of specific transporters in the translocation of the PS-NPs across the BeWo b30 cell layer, translocation studies were also performed in the absence and presence of transporter inhibitors. The presence of PgP, BCRP, MRP-1 has been confirmed in the BeWo b30 cells (Atkinson et al. 2003, Ceckova et al. 2006, Evseenko et al. 2006, Milane et al. 2009, Mitra and Audus 2010, Wick et al. 2010) but inhibitors of PgP and BCRP at a concentration of 50 and $2.5 \mu \mathrm{M}$, respectively, showed no effect on translocation of PS-NPs across the BeWo b30 cell layers. MK571 $(24 \mu \mathrm{M})$, an inhibitor of MRP-1, significantly increased the basolateral translocation of positively charged PS-NP by 1.5 fold but did not influence the translocation of the negatively charged PS-NPs so in general the translocation of the PS-NPs tested appeared to occur preferentially by passive diffusion.

In vitro models are attractive for high throughput testing of various NPs. However, it should be kept in mind that in vitro models like the BeWo b30 transwell model applied in the current study have their limitations (Nikitina et al. 2013). For example, given that the physiology of the placental barrier in vivo varies in time, it is difficult to classify the BeWo cell layer in a representing specific stage of pregnancy. Furthermore, the BeWo transwell model is a simplified placental barrier compared to the complex in vivo placental barrier since it lacks the multiple layers of different cell types (cytotrophoblasts and syncytiotrophoblasts) and extracellular matrix including collagen, known to constitute the in vivo placental barrier. This implies that extrapolation of in vitro results to the in vivo situation should acknowledge uncertainties in the predictions made (Mathiesen et al. 2014). To this end we have included the model compounds amoxicillin and antipyrine, known to be translocated across the placental barrier to respectively only a limited or large extent (Akbaraly et al. 1985, Li et al. 2013) to enable qualitative interpretation of the results. This comparison revealed that for positively charged PSNPs translocation was 17 fold lower and for negatively charged PS-NPs from Polysciences 2.5 fold higher than that of amoxicillin, while for the negatively charged PS-NPs from Magsphere no translocation was found under the same test conditions.

In conclusion, the translocation studies of PS-NPs in the present study and the comparison of the data with translocation data of model compounds, for which the model has been validated with an ex vivo placental model, reveal that the in vitro BeWo b30 model can be used as a fast method to get an initial qualitative impression about the capacity of NPs to translocate across the placental barrier and set priorities for further in vivo studies on translocation of NPs to the fetus. 
Chapter 3

\section{ACKNOWLEDGEMENTS}

This work is supported by NanoNextNL, a micro and nanotechnology consortium of the Government of the Netherlands and 130 partners. 


\section{REFERENCES}

Akbaraly, J. P., S. Guibert, J. J. Leng and J. Auzerie (1985). "Transplacental transfer of 5 antibiotics by in vitro human placental perfusion." Pathol Biol (Paris) 33(5): 368-372.

Ali, H., I. Kalashnikova, M. A. White, M. Sherman and E. Rytting (2013). "Preparation, characterization, and transport of dexamethasone-loaded polymeric nanoparticles across a human placental in vitro model." Int J Pharm 454(1): 149-157.

Arora, A. and G. W. Padua (2010). "Review: nanocomposites in food packaging." J Food Sci 75(1): R43-49.

Artursson, P. and J. Karlsson (1991). "Correlation between oral drug absorption in humans and apparent drug permeability coefficients in human intestinal epithelial (Caco-2) cells." Biochem Biophys Res Commun 175(3): 880-885.

Atkinson, D. E., S. L. Greenwood, C. P. Sibley, J. D. Glazier and L. J. Fairbairn (2003). "Role of MDR1 and MRP1 in trophoblast cells, elucidated using retroviral gene transfer." American journal of physiology. Cell physiology 285(3): C584-591.

Auffan, M., J. Rose, J. Y. Bottero, G. V. Lowry, J. P. Jolivet and M. R. Wiesner (2009). "Towards a definition of inorganic nanoparticles from an environmental, health and safety perspective." Nat Nanotechnol 4(10): 634-641.

Avery, M. L., C. E. Meek and K. L. Audus (2003). "The presence of inducible cytochrome P450 types 1A1 and 1A2 in the BeWo cell line." Placenta 24(1): 45-52.

Bhattacharjee, S., D. Ershov, K. Fytianos, J. Gucht, G. Alink, I. Rietjens, A. Marcelis and H. Zuilhof (2012). "Cytotoxicity and cellular uptake of tri-block copolymer nanoparticles with different size and surface characteristics." Particle and Fibre Toxicology 9(1): 11.

Bhattacharjee, S., D. Ershov, J. V. Gucht, G. M. Alink, I. M. Rietjens, H. Zuilhof and A. T. Marcelis (2011). "Surface charge-specific cytotoxicity and cellular uptake of tri-block copolymer nanoparticles." Nanotoxicology.

Bhattacharjee, S., I. M. Rietjens, M. P. Singh, T. M. Atkins, T. K. Purkait, Z. Xu, S. Regli, A. Shukaliak, R. J. Clark, B. S. Mitchell, G. M. Alink, A. T. Marcelis, M. J. Fink, J. G. Veinot, S. M. Kauzlarich and H. Zuilhof (2013). "Cytotoxicity of surface-functionalized silicon and germanium nanoparticles: the dominant role of surface charges." Nanoscale 5(11): 4870-4883.

Bhattacharjee, S., E. J. van Opstal, G. M. Alink, A. T. M. Marcelis, H. Zuilhof and I. M. C. M. Rietjens (2013). "Surface charge-specific interactions between polymer nanoparticles and ABC transporters in Caco-2 cells." Journal of Nanoparticle Research 15(6).

Bode, C. J., H. Jin, E. Rytting, P. S. Silverstein, A. M. Young and K. L. Audus (2006). "In vitro models for studying trophoblast transcellular transport." Methods Mol Med 122: 225-239.

Brand, W., P. A. van der Wel, M. J. Rein, D. Barron, G. Williamson, P. J. van Bladeren and I. M. Rietjens (2008). "Metabolism and transport of the citrus flavonoid hesperetin in Caco-2 cell monolayers." Drug metabolism and disposition: the biological fate of chemicals 36(9): 1794-1802.

Buerki-Thurnherr, T., M. von and P. Wick (2012). "Knocking at the door of the unborn child: engineered nanoparticles at the human placental barrier." Swiss medical weekly 142: 0.

Cartwright, L., M. S. Poulsen, H. M. Nielsen, G. Pojana, L. E. Knudsen, M. Saunders and E. Rytting (2012). "In vitro placental model optimization for nanoparticle transport studies." Int J Nanomedicine 7: 497-510.

Ceckova, M., A. Libra, P. Pavek, P. Nachtigal, M. Brabec, R. Fuchs and F. Staud (2006). "Expression and functional activity of breast cancer resistance protein (bcrp, abcg2) transporter in the human choriocarcinoma cell line bewo" Clinical and Experimental Pharmacology and Physiology 33(1-2): 58-65.

Chiu, H. W., T. Xia, Y. H. Lee, C. W. Chen, J. C. Tsai and Y. J. Wang (2015). "Cationic polystyrene nanospheres induce autophagic cell death through the induction of endoplasmic reticulum stress." Nanoscale 7(2): 736-746.

Correia Carreira, S., L. Walker, K. Paul and M. Saunders (2013). "The toxicity, transport and uptake of nanoparticles in the in vitro BeWo b30 placental cell barrier model used within NanoTEST." Nanotoxicology.

dos Santos, T., J. Varela, I. Lynch, A. Salvati and K. A. Dawson (2011). "Effects of Transport Inhibitors on the Cellular Uptake of Carboxylated Polystyrene Nanoparticles in Different Cell Lines." PLoS One 6(9): e24438.

Evseenko, D. A., J. W. Paxton and J. A. Keelan (2006). "ABC drug transporter expression and functional activity in trophoblast-like cell lines and differentiating primary trophoblast." American Journal of Physiology - Regulatory, Integrative and Comparative Physiology 290(5): R1357-R1365. 
Grafmuller, S., P. Manser, H. F. Krug, P. Wick and U. von Mandach (2013). "Determination of the transport rate of xenobiotics and nanomaterials across the placenta using the ex vivo human placental perfusion model." J Vis $\operatorname{Exp}(76)$.

Juch, H., L. Nikitina, P. Debbage, G. Dohr and M. Gauster (2013). "Nanomaterial interference with early human placenta: Sophisticated matter meets sophisticated tissues." Reproductive Toxicology 41(0): 73-79.

Kim, W. J., A. C. Bonoiu, T. Hayakawa, C. Xia, M. A. Kakimoto, H. E. Pudavar, K. S. Lee and P. N. Prasad (2009). "Hyperbranched polysiloxysilane nanoparticles: surface charge control of nonviral gene delivery vectors and nanoprobes." Int J Pharm 376(1-2): 141-152.

Lee, J. Y., J. S. Kim, H. J. Cho and D. D. Kim (2014). "Poly(styrene)-b-poly(DL-lactide) copolymer-based nanoparticles for anticancer drug delivery." Int J Nanomedicine 9: 2803-2813.

Lesniak, A., A. Campbell, M. P. Monopoli, I. Lynch, A. Salvati and K. A. Dawson (2010). "Serum heat inactivation affects protein corona composition and nanoparticle uptake." Biomaterials 31(36): 9511-9518.

Li, H., B. van Ravenzwaay, I. M. Rietjens and J. Louisse (2013). "Assessment of an in vitro transport model using BeWo b30 cells to predict placental transfer of compounds." Arch Toxicol 87(9): 1661-1669.

Liu, F., M. J. Soares and K. L. Audus (1997). "Permeability properties of monolayers of the human trophoblast cell line BeWo." American Journal of Physiology - Cell Physiology 273(5): C1596-C1604.

Liu, W., J. Rose, S. Plantevin, M. Auffan, J. Y. Bottero and C. Vidaud (2013). "Protein corona formation for nanomaterials and proteins of a similar size: hard or soft corona?" Nanoscale 5(4): 1658-1668.

Liu, X., N. Huang, H. Li, Q. Jin and J. Ji (2013). "Surface and Size Effects on Cell Interaction of Gold Nanoparticles with Both Phagocytic and Nonphagocytic Cells." Langmuir 29(29): 9138-9148.

Lundqvist, M., J. Stigler, G. Elia, I. Lynch, T. Cedervall and K. A. Dawson (2008). "Nanoparticle size and surface properties determine the protein corona with possible implications for biological impacts." Proceedings of the National Academy of Sciences 105(38): 14265-14270.

Mathiesen, L., T. A. Morck, G. Zuri, M. H. Andersen, C. Pehrson, M. Frederiksen, T. Mose, E. Rytting, M. S. Poulsen, J. K. Nielsen and L. E. Knudsen (2014). "Modelling of Human Transplacental Transport as Performed in Copenhagen, Denmark." Basic \& clinical pharmacology \& toxicology.

Menjoge, A. R., A. L. Rinderknecht, R. S. Navath, M. Faridnia, C. J. Kim, R. Romero, R. K. Miller and R. M. Kannan (2011). "Transfer of PAMAM dendrimers across human placenta: prospects of its use as drug carrier during pregnancy." J Control Release 150(3): 326-338.

Milane, A., S. Vautier, H. Chacun, V. Meininger, G. Bensimon, R. Farinotti and C. Fernandez (2009). "Interactions between riluzole and ABCG2/BCRP transporter." Neuroscience Letters 452(1): 12-16.

Mitra, P. and K. L. Audus (2010). "MRP isoforms and BCRP mediate sulfate conjugate efflux out of BeWo cells." International Journal of Pharmaceutics 384(1-2): 15-23.

Myllynen, P. K., M. J. Loughran, C. V. Howard, R. Sormunen, A. A. Walsh and K. H. Vahakangas (2008). "Kinetics of gold nanoparticles in the human placenta." Reprod Toxicol 26(2): 130-137.

Nikitina, L., G. Dohr and H. Juch (2013). "Studying nanoparticle interaction with human placenta: Festina lente!" Nanotoxicology.

Oberdorster, E. (2004). "Manufactured nanomaterials (fullerenes, C60) induce oxidative stress in the brain of juvenile largemouth bass." Environ Health Perspect 112(10): 1058-1062.

Oberdorster, G., E. Oberdorster and J. Oberdorster (2005). "Nanotoxicology: an emerging discipline evolving from studies of ultrafine particles." Environ Health Perspect 113(7): 823-839.

Park, M. V., A. M. Neigh, J. P. Vermeulen, L. J. de la Fonteyne, H. W. Verharen, J. J. Briede, H. van Loveren and W. H. de Jong (2011). "The effect of particle size on the cytotoxicity, inflammation, developmental toxicity and genotoxicity of silver nanoparticles." Biomaterials 32(36): 9810-9817.

Phosphorex Commercial Applications Use Polystyrene Nanoparticles. 2013 ; Available from: http://www.phosphorex.com/1/post/2013/07/commercial-applications-use-polystyrene-nanoparticles.html.

Poulsen, M. S., E. Rytting, T. Mose and L. E. Knudsen (2009). "Modeling placental transport: correlation of in vitro BeWo cell permeability and ex vivo human placental perfusion." Toxicol In Vitro 23(7): 1380-1386.

Sager, T. M. and V. Castranova (2009). "Surface area of particle administered versus mass in determining the pulmonary toxicity of ultrafine and fine carbon black: comparison to ultrafine titanium dioxide." Part Fibre Toxicol 6: 15.

Saunders, M. (2009). "Transplacental transport of nanomaterials." Wiley Interdiscip Rev Nanomed Nanobiotechnol 1(6): 671-684. 
Simon, P., Q. Chaudhry and D. Bakos (2008). "Migration of engineered nanoparticles from polymer packaging to food - a physicochemical view." J Food Nutr Res 47(3): 105-113.

Sonnegaard Poulsen, M., T. Mose, L. Leth Maroun, L. Mathiesen, L. Ehlert Knudsen and E. Rytting (2013). "Kinetics of silica nanoparticles in the human placenta." Nanotoxicology.

Stampfl, A., M. Maier, R. Radykewicz, P. Reitmeir, M. Göttlicher and R. Niessner (2011). "Langendorff Heart: A Model System To Study Cardiovascular Effects of Engineered Nanoparticles." ACS Nano 5(7): 5345-5353.

Takeuchi, Y., R. Sakakibara and M. Ishiguro (1990). "Synthesis and secretion of human chorionic gonadotropin and its subunits in choriocarcinoma cells: a comparative study with normal placental cells." Mol Cell Endocrinol 69(2-3): 145-156.

Ushigome, F., H. Takanaga, H. Matsuo, S. Yanai, K. Tsukimori, H. Nakano, T. Uchiumi, T. Nakamura, M. Kuwano, H. Ohtani and Y. Sawada (2000). "Human placental transport of vinblastine, vincristine, digoxin and progesterone: contribution of P-glycoprotein." European Journal of Pharmacology 408(1): 1-10.

Utoguchi, N., G. A. Chandorkar, M. Avery and K. L. Audus (2000). "Functional expression of P-glycoprotein in primary cultures of human cytotrophoblasts and BeWo cells." Reprod Toxicol 14(3): 217-224.

Walczak, A. P., E. Kramer, P. J. Hendriksen, P. Tromp, J. P. Helsper, M. van der Zande, I. M. Rietjens and H. Bouwmeester (2014). "Translocation of differently sized and charged polystyrene nanoparticles in in vitro intestinal cell models of increasing complexity." Nanotoxicology: 1-9.

Wick, P., A. Malek, P. Manser, D. Meili, X. Maeder-Althaus, L. Diener, P. A. Diener, A. Zisch, H. F. Krug and U. von Mandach (2010). "Barrier capacity of human placenta for nanosized materials." Environmental health perspectives 118(3): 432-436. 


\section{SUPPLEMENTARY MATERIALS CHAPTER 3}

Table S1. Transporter inhibitors used in the PS-NP distribution studies.

\begin{tabular}{llll}
\hline Transporter inhibitor & Target & $\begin{array}{l}\text { Concentration } \\
\text { used in present } \\
\text { study }(\mu \mathrm{M})\end{array}$ & Reference \\
\hline Methyl- $\beta$-cyclodextrin & Caveolin & 1 & (Bhattacharjee et al. 2013) \\
Chlorpromazine & Clathrin & 5 & (dos Santos et al. 2011) \\
Verapamil & PgP & 50 & (Ushigome et al. 2000) \\
KO143 & BCRP & 2.5 & (Brand et al. 2008) (Ceckova et al. 2006) \\
MK571 & MRP-1/MRP-2 & 24 & (Brand et al. 2008) \\
\hline
\end{tabular}

Table S2. Automatic diameter measurements of PS-NPs using Scandium SIS image analysis software (Olympus Soft Imaging Solutions GmbH, Germany). The mean diameter \pm SD and range of 50-500 particles is given (in $\mathrm{nm}$ ).

\begin{tabular}{lccc}
\hline Nanoparticle & Mean & SD & Range \\
\hline 50 nm negatively charged PS-NP (Polysciences) & 31.6 & 13.6 & $15-70$ \\
$50 \mathrm{~nm}$ negatively charged PS-NP (Magsphere) & 35.0 & 15.3 & $15-70$ \\
$50 \mathrm{~nm}$ positively charged PS-NP (Magsphere) & 50.6 & 9.3 & $25-75$ \\
\hline
\end{tabular}



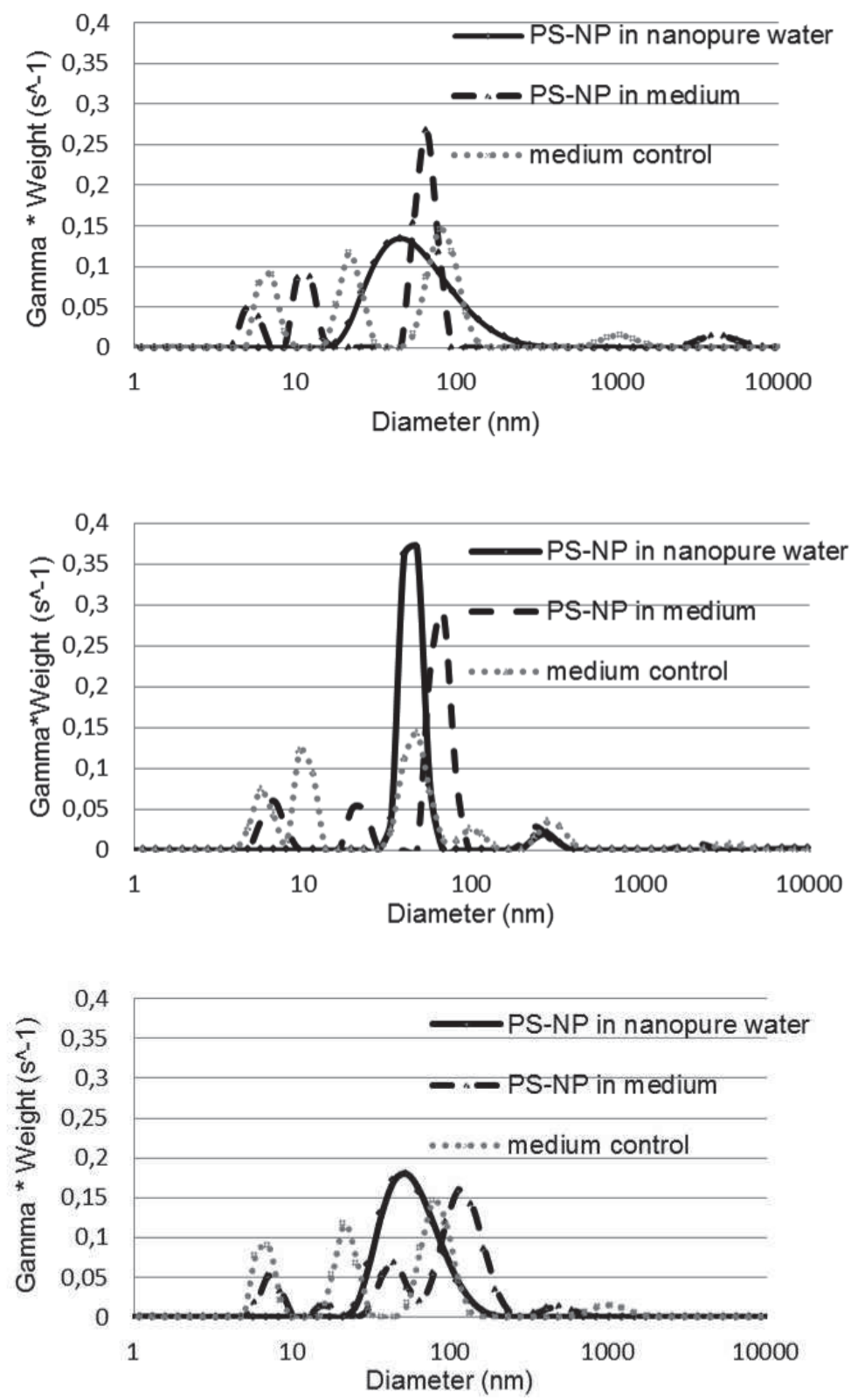

Figure S1. Dynamic light scattering of A) $50 \mathrm{~nm}$ positively charged PS-NP (Magsphere), B) $50 \mathrm{~nm}$ negatively charged PS-NP (Magsphere), C) $50 \mathrm{~nm}$ negatively charged PS-NP (Polysciences) freshly dispersed at $10 \mu \mathrm{g} / \mathrm{ml}$ in cell culture medium or nanopure water; for comparison the scattering of the cell culture medium alone is also shown (medium control). 


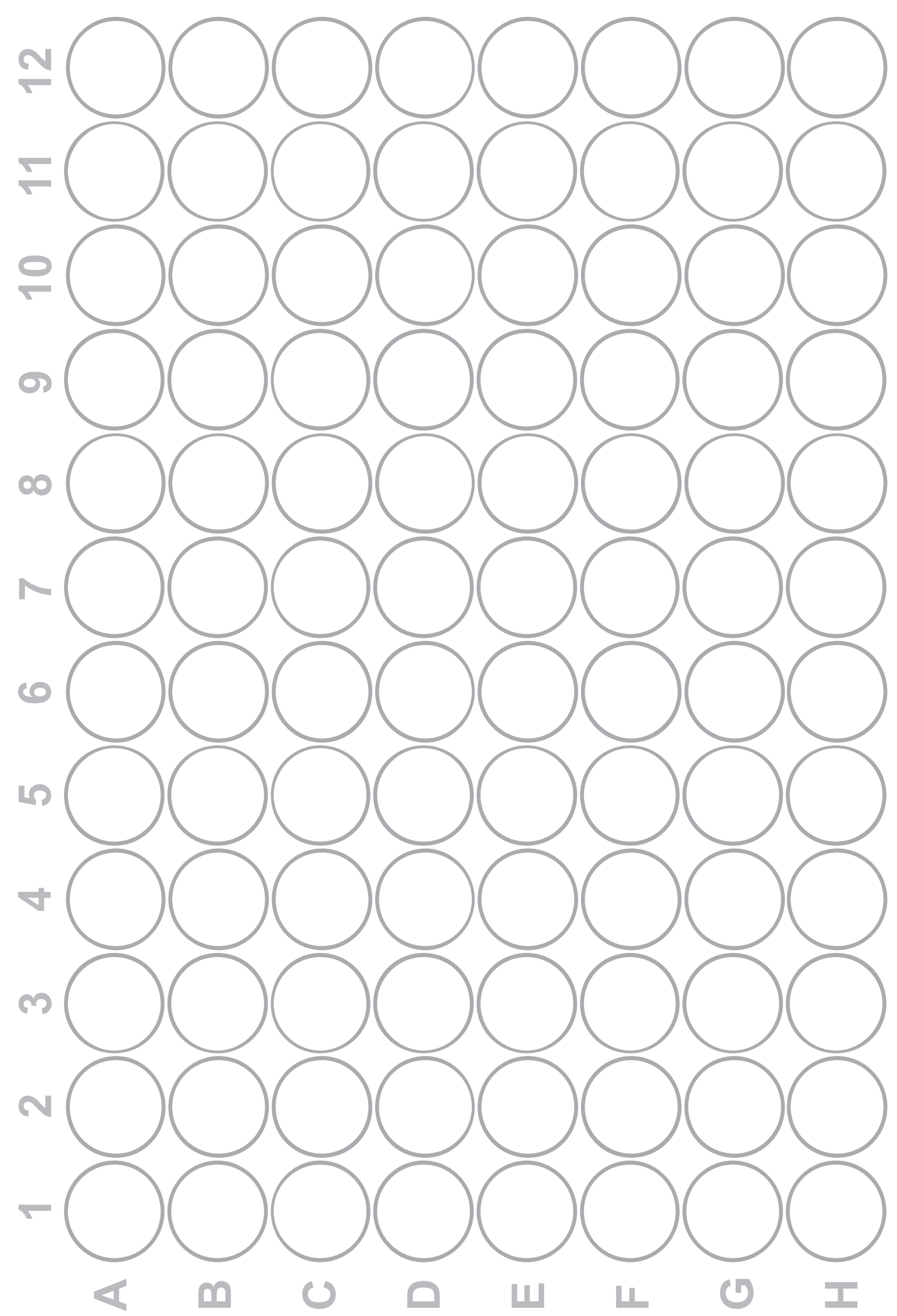




\section{Chapter 4}

\section{Assessment of the potential developmental toxicity of positively and negatively charged polystyrene nanoparticles using the embryonic stem cell differentiation assay}

Samantha K. Kloet Hans H.J. van den Berg Leonie F.H. Fransen Ignacio Miro Estruch

Vasileios Vasileiadis

Laura de Haan Ivonne M.C.M. Rietjens Jochem Louisse 


\section{ABSTRACT}

Knowledge on the relation between physicochemical characteristics of nanoparticles (NPs) and their toxicity would be of great help for setting priorities for NP toxicity testing. The present study aimed to assess the role of surface charge of NPs on in vitro developmental toxicity, by determining the effects of positively or negatively charged polystyrene nanoparticles (PS-NPs) in the embryonic stem (ES-D3) cell differentiation assay. The results show that the negatively charged PS-NPs did not affect ES-D3 cell differentiation, whereas the positively charged PS-NPs showed a concentration-dependent inhibition of ES-D3 cell differentiation, indicating that the effects of NPs on ES-D3 cell differentiation may depend on their surface charge. In order to assess whether NP-induced effects on ES-D3 cell differentiation may be related to other physicochemical properties, we performed a literature search on the reported potencies of NPs in these models. This analysis shows that the potency of NPs may depend on size, surface charge or coating, but that currently available data are too limited to indicate a clear relation between physicochemical characteristics and their in vitro developmental toxicity. The results also reveal that additional experimental data, as presented in the present study, may help to further advance the field. 


\section{INTRODUCTION}

The rapid increase of nanotechnology has several benefits for society, yet there is an increasing concern that exposure to nanoparticles (NPs) may result in significant adverse effects (Oberdorster et al. 2007, Gebel et al. 2013). NPs have been shown to be able to pass physiological barriers, such as that of the intestines and the placenta (Braakhuis et al. 2015). This indicates that systemic exposure, including fetal exposure, is feasible and that the fetus is a potential target for NPs. To assess possible adverse effects of NPs on the developing fetus, in vivo developmental toxicity studies can be performed in for example rats or mice. However, because of economic and ethical constrains with in vivo studies, alternatives for in vivo toxicity studies are being developed and validated (Jelinek et al. 1985, Fort and Paul 2002, zur Nieden et al. 2004, Spielmann et al. 2006, Park et al. 2009, de Jong et al. 2011).

One of the in vitro alternative assays for developmental toxicity testing is the embryonic stem cell test (EST) which has been validated by the European Centre for the Validation of Alternative Methods (ECVAM). In particular the differentiation assay of the EST has been shown to be useful in assessing relative developmental toxicity potencies of chemicals and NPs (Genschow et al. 2004, de Jong et al. 2011, Pietroiusti et al. 2011, Li et al. 2015). In this assay, the chemical- or NP-induced inhibition of blastocyst-derived mouse embryonic stem (ES-D3) cells developing into contracting cardiomyocytes, is used as an in vitro endpoint of developmental toxicity (de Jong et al. 2009, Campagnolo et al. 2013). Considering the large variety of NPs that have not been tested yet for their developmental toxicity, knowledge on the relation between physicochemical characteristics of NPs, such as size or surface charge, and their toxicity would be of great help for setting priorities for NP toxicity testing.

To increase our knowledge on the relation between physicochemical properties of NPs and their developmental toxicity potential, the present study aimed to assess the role of surface charge in the in vitro developmental toxicity potential of NPs. To this end, we assessed the effects of one $50 \mathrm{~nm}$ positively charged polystyrene NP (PS-NP) and two $50 \mathrm{~nm}$ negatively charged PS-NPs on the viability and differentiation of ES-D3 cells. Further, we made an overview of the available literature on NP-induced effects on ES-D3 cell differentiation, in order to assess whether also other physicochemical characteristics may relate to the in vitro developmental toxicity potential of NPs.

\section{METHODS}

\section{Materials}

WST-1 Cell Proliferation Reagent was purchased from Roche (Woerden, The Netherlands). Phosphate buffered saline (PBS), non-essential amino acids, penicillin-streptomycin and 
L-glutamine were obtained from Invitrogen (Breda, The Netherlands). Non-enzymatic cell dissociation solution, $0.1 \%$ gelatine, murine leukemia inhibiting factor (LIF) and $\beta$-mercaptoethanol were purchased from Sigma-Aldrich (Zwijndrecht, The Netherlands). AdvanceSTEM $^{\mathrm{TM}}$ Low Osmo DMEM was obtained from Hyclone (Hyclone; Logan, USA) and foetal calf serum (FCS) was obtained from ATCC (USA).

\section{Nanoparticles}

This study included three different PS-NPs, one $50 \mathrm{~nm}$ positively charged PS-NP and two $50 \mathrm{~nm}$ negatively charged PS-NPs. Positively and negatively charged fluorescent red PS-NPs were obtained from Magsphere Inc. (California, USA) (Cat nr: CAFR050NM, AMFR050NM respectively). Negatively charged fluorescent Yellow-Green carboxylated $50 \mathrm{~nm}$ PS-NPs were obtained from Polysciences (Warrington, USA) (Cat nr. 16661-10). All the PS-NPs used had an average particle size of $50 \mathrm{~nm}$ as indicated by the manufacturers' specification.

\section{Particle characterization}

The size (hydrodynamic diameter) of the NPs was determined by dynamic light scattering (DLS) using a Cobolt Samba 300mW DPSS laser at a wavelength of $532 \mathrm{~nm}$ as a light source. Measurements were performed on freshly prepared NP solutions at $100 \mu \mathrm{g} / \mathrm{ml}$ in nanopure water and in cell culture medium containing $20 \%$ FCS. For each sample, twenty measurements were made and the detector intensity data (measured with an ALV/SO SIPD Single Photon Detector with ALV Static and Dynamic Fiber optics) were processed with an ALV5000/60X0 external correlator and ALV-5000/E software (all from ALV-GmbH, Germany). Data were analysed with the AfterALV software and mean diameter and polydispersity index (PDI) were determined for each NP sample. Additionally, the $\zeta$-potential of the NPs was measured at a concentration of $100 \mu \mathrm{g} / \mathrm{ml}$ using the Malvern Zetasizer 2000 (Malvern Instruments, UK).

\section{Cell culture}

The murine ES-D3 cell line was purchased from ATCC (Wesel, Germany). The cells were maintained in cell culture flasks (Corning, the Netherlands) that were pre-coated with $0.1 \%$ gelatin, using AdvanceSTEM ${ }^{\mathrm{TM}}$ Low Osmo DMEM, supplemented with 20\% FCS, $2 \mathrm{mM}$ L-glutamine and 50U/50 $\mu$ g penicillin-streptomycin and leukemia inhibiting factor (LIF 1000 $\mathrm{U} / \mathrm{ml}$ ), in a humidified atmosphere at $37^{\circ} \mathrm{C}$ and $5 \% \mathrm{CO}_{2}$. LIF serves to keep the embryonic stem cells in the pluripotent state, by inhibiting their differentiation. Cells were subcultured every 2 to 3 days using non-enzymatic cell dissociation solution to detach the cells when reaching $60-80 \%$ confluence. Experiments were performed using ES-D3 cells between passages 4 and 18 . 


\section{Assessment of cell viability}

To assess the effects of the PS-NPs on cell viability, ES-D3 cells were seeded at $2 \times 10^{4}$ cells/ $\mathrm{ml}$ (one-day exposure) or $1 \times 10^{3}$ cells/ml (five-day exposure) in $100 \mu \mathrm{L}$ culture medium in 96-well plates (Greiner bio-one) in the absence of LIF and incubated at $37^{\circ} \mathrm{C}$ and $5 \% \mathrm{CO}_{2}$ for 24 hours to allow cell adherence. Then the cells were exposed to $100 \mu \mathrm{L}$ of PS-NPs at concentrations up to $100 \mu \mathrm{g} / \mathrm{ml}$ and subsequently incubated for one or five days at $37^{\circ} \mathrm{C}$ and $5 \%$ $\mathrm{CO}_{2}$ in a humidified atmosphere. Culture medium was used as a negative control and $200 \mu \mathrm{M}$ $\mathrm{CuSO}_{4}$ was used as a positive control. After incubation, $20 \mu \mathrm{L}$ WST-1 reagent was added to each well and the plates were incubated for another 3 hours. Then absorbance was measured at $450 \mathrm{~nm}$ and $620 \mathrm{~nm}$ (background) using a SpectraMax M2 Microplate Reader (Molecular Devices LLC, Sunnyvale, CA USA). The cell viability was expressed as percentage of the negative control, with the control set at $100 \%$ viability.

\section{Differentiation assay with ES-D3 cells}

The potential of PS-NPs to inhibit ES-D3 cell differentiation was evaluated using the ES-D3 cell differentiation assay using culture medium without LIF. In this assay, the effect of chemicals or NPs is assessed on the differentiation of ES-D3 cells into contracting cardiomyocytes. Similar to other studies (van Dartel et al. 2009, van Dartel et al. 2009, van Dartel et al. 2011), we exposed differentiating ES-D3 cells during day 3-10 of the 10-day differentiation process. On day 0, droplets of $20 \mu \mathrm{l}$ ES-D3 cell suspension $\left(3.75 \times 10^{4}\right.$ cells $\left./ \mathrm{ml}\right)$ in the absence of PSNPs were placed as hanging drops on the inner side of the lid of a 96-well plate. Sterile lids of Eppendorf tubes were placed on each corner of the plate to prevent contact of the drops with the plate. The wells of the 96-well plate were filled with $250 \mu \mathrm{l}$ PBS and the plate was sealed with Micropore tape (3M, Neuss, Germany) to prevent evaporation of the hanging drops. Plates were incubated for three days at $37^{\circ} \mathrm{C}$ and $5 \% \mathrm{CO}_{2}$ in a humidified atmosphere to allow for the formation of embryonic bodies (EBs). These EBs were transferred after 3 days to non-tissue culture treated Petri dishes (Greiner, diameter $6 \mathrm{~cm}$ ) with $5 \mathrm{ml}$ of medium in the absence (negative control) or presence of PS-NPs. On day 5, the EBs were transferred to a 24-well plate (Corning) with $1 \mathrm{ml}$ of medium in the absence or presence of PS-NPs, plating one EB per well. On day 10, the number of wells containing contracting cardiomyocytes was determined by visual inspection using a light microscope. Tests were accepted for further analysis if at least 21 of the 24 wells of the negative control contained contracting cardiomyocytes. For each test compound, three independent assays were performed, at concentrations ranging from 0.1 to $50 \mu \mathrm{g} / \mathrm{ml}$. For each concentration, the fraction of the EBs that differentiated into contracting cardiomyocytes was determined, with a value of 1 implying that all EBs in one 24-well plate differentiated into contracting cardiomyocytes. 


\section{Data analysis}

For each study, three independent experiments were performed. In each cell viability study, three technical replicates were used for each condition. Data are shown as mean \pm standard deviation (SD) of all data points and plotted in graphs using GraphPad Prism ${ }^{\circledR}$ version 5. Concentration-response analysis was performed using GraphPad Prism version 5.

\section{Literature search on reported effects of NPs in the ES-D3 differentiation assay}

A literature search was performed to obtain an overview of the effects of NPs on ES-D3 cell differentiation, in order to assess whether the NP-induced inhibition of ES-D3 cell differentiation may be related to specific physicochemical characteristics. For this literature search the following search terms were used in Pubmed and Scopus databases: mouse embryonic stem cell, ES-D3, embryonic stem cell test, EST, ES-D3 cell differentiation assay, nanoparticle, nanomaterial, in vitro, developmental toxicity.

\section{RESULTS}

\section{Physicochemical characterization of the NPs}

This study included three different PS-NPs, one $50 \mathrm{~nm}$ positively charged PS-NP and two 50 $\mathrm{nm}$ negatively charged PS-NPs. The hydrodynamic diameters of the NPs were measured by DLS in nanopure water and in the culture medium containing 20\% FCS (Table 1). The measured sizes of the PS-NPs in nanopure water were close to the sizes reported by the manufacturer. Upon addition of the PS-NPs to DMEM with 20\% FCS, a slight increase in size was observed (Table 1). The PDI increased upon addition of the PS-NPs to DMEM with 20\% FCS as compared to the PDI in water, indicating that the size distribution became wider. Background DLS values of DMEM with 20\% FCS and water are 19.9 and $0 \mathrm{~nm}$ respectively (Table 1).

Table 1. Dynamic light scattering (DLS) $(n=3)$ and zeta potential $(n=3)$ results.

\begin{tabular}{lcccc}
\hline NP & \multicolumn{2}{c}{ Diameter in $\mathbf{n m} \pm$ SD (PDI) } & \multicolumn{2}{c}{ Zeta potential (mV) \pm SD } \\
& In water & $\begin{array}{c}\text { DMEM with 20\% } \\
\text { FCS }\end{array}$ & $\begin{array}{c}\text { In water } \\
\text { DMEM with 20\% } \\
\text { FCS }\end{array}$ \\
\hline $\begin{array}{l}50 \text { nm positively charged PS-NP } \\
\text { (Magsphere) }\end{array}$ & $56.5 \pm 3.0(0.09)$ & $66.6 \pm 2.4(0.34)$ & $51.0 \pm 4.5$ & $-8.7 \pm 0.2$ \\
$\begin{array}{l}50 \text { nm negatively charged PS-NP } \\
\text { (Magsphere) }\end{array}$ & $50.4 \pm 1.7(0.19)$ & $60.9 \pm 5.4(0.33)$ & $-55.5 \pm 6.7$ & $-7.8 \pm 0.9$ \\
$\begin{array}{l}50 \text { nm negatively charged PS-NP } \\
\text { (Polysciences) }\end{array}$ & $52.4 \pm 0.1(0.21)$ & $55.7 \pm 4.8(0.32)$ & $-58.3 \pm 2.7$ & $-9.7 \pm 1.1$ \\
None & 0 & $19.9 \pm 1.2(0.46)$ & 0 & $-9.2 \pm 1.3$ \\
\hline
\end{tabular}


The surface charges of the positively and negatively charged PS-NPs were measured in nanopure water and in DMEM with 20\% FCS by determining the zeta potential (Table 1). Positively charged PS-NPs had a zeta potential of $+51.0 \mathrm{mV}$ in nanopure water. Negatively charged PS-NPs of Magsphere and Polyscience had a zeta potential of $-55.5 \mathrm{mV}$ and $-58.3 \mathrm{mV}$, respectively, in nanopure water. In DMEM with $20 \%$ FCS all NPs got a slightly negative charge of -8 to $-9 \mathrm{mV}$, similar to the charge measured for DMEM with $20 \%$ FCS without PS-NPs (Table 1).

\section{Effects of PS-NPs on ES-D3 cell viability and ES-D3 cell differentiation}

To evaluate the effects of the PS-NPs on ES-D3 cell viability, the WST-1 assay was performed on ES-D3 cells exposed for one day or five days to the PS-NPs (Figure 1). The positively charged PS-NPs caused a concentration-dependent decrease in cell viability, which was more prominent after the 5-day exposure than after the 1-day exposure (Figure 1A), indicated by EC50 values of 2.0 and $71 \mu \mathrm{g} / \mathrm{ml}$, respectively. For both negatively charged PS-NPs no effect on cell viability was observed up to the highest concentrations tested $(100 \mu \mathrm{g} / \mathrm{ml})$ (Figure 1B and $1 \mathrm{C})$.

To study the in vitro developmental toxicity of the PS-NPs, their effect on the differentiation of ES-D3 cells into contracting cardiomyocytes was evaluated (Figure 1). The positively charged PS-NPs showed a concentration-dependent inhibition of the differentiation of ES-D3 cells into beating cardiomyocytes, with an EC50 of $1.2 \mu \mathrm{g} / \mathrm{ml}$, whereas both the negatively charged PS-NPs did not affect the differentiation of the ES-D3 cells up to the highest concentration tested $(50 \mu \mathrm{g} / \mathrm{ml})$ (Figure $1 \mathrm{~B}$ and $1 \mathrm{C})$.

The results indicate a clear effect of NP charge on both viability and differentiation of the ES-D3 cells. The results for the negatively charged PS-NPs do not seem to point at a hazard for developmental toxicity of these NPs. The results for the positively charged PS-NPs may indicate a possible hazard for developmental toxicity. Since cell differentiation was inhibited at concentrations that also inhibited cell viability upon a 5-day exposure, NP-induced inhibition of ES-D3 cell differentiation may be due to cytotoxic effects. 

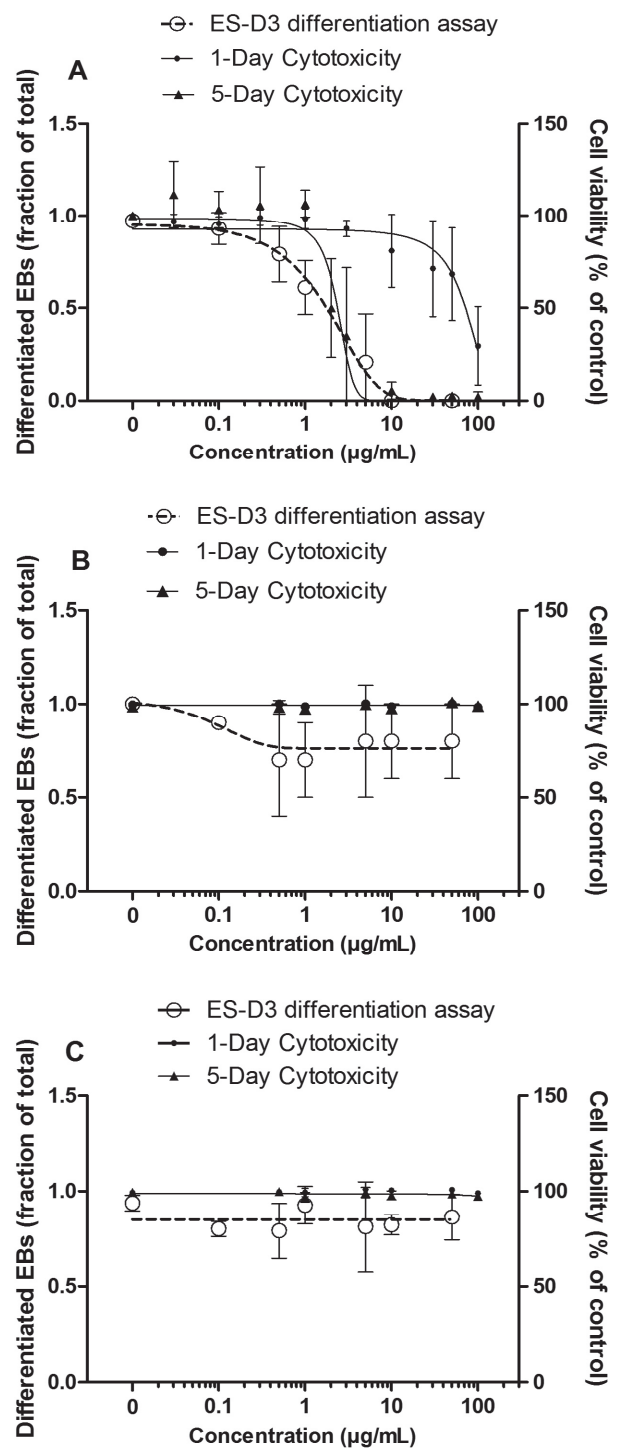

Figure 1. Concentration-dependent effects of A) $50 \mathrm{~nm}$ positively charged PS-NPs (Magsphere), B) $50 \mathrm{~nm}$ negatively charged PS-NPs (Magsphere) C) $50 \mathrm{~nm}$ negatively charged PSNPs (Polysciences) on ES-D3 cell viability (1-day exposure: with solid line; 5-day exposure: $\Delta$ with solid line) and ES-D3 cell differentiation into beating cardiomyocytes ( $\bigcirc$ with dotted line). Cell viability data are expressed as percentage of the negative control and cell differentiation data are expressed as the fraction differentiated EBs of the total EBs for that exposure condition. Data are presented as the mean \pm SD of $n=3$ independent biological replicates. 


\section{Reported effects of other NPs on ES-D3 cell differentiation}

In order to obtain further insight in the role of physicochemical characteristics of NPs on their potential developmental toxicity, we performed a literature search on the NP-induced inhibition of ES-D3 cell differentiation into contracting cardiomyocytes. Table 2 presents an overview of the literature data obtained, showing the EC50 values for the inhibition of ES-D3 cell differentiation, including EC50 values for cell viability, as well as the NP physicochemical properties.

For silicon dioxide NPs and silver NPs, different sizes have been tested in the ES-D3 cell differentiation assay. For silicon dioxide NPs, the two largest particles (80 and $400 \mathrm{~nm}$ ) did not inhibit ES-D3 cell differentiation up to the highest concentration tested $(100 \mu \mathrm{g} / \mathrm{ml})$, whereas the two smallest particles (10 and $30 \mathrm{~nm}$ ) inhibited ES-D3 cell differentiation with EC50 values of 59 and $29 \mu \mathrm{g} / \mathrm{ml}$, respectively (Table 2). For silver NPs all four sizes (7, 20, 80 and 113 $\mathrm{nm}$ ) inhibited ES-D3 cell differentiation, and the smaller the NPs, the higher their potency, except for the two largest particles, which showed a similar potency (Table 2). For other NPs, the effect of coating on the in vitro developmental toxicity has been assessed. Table 2 shows that the coating of NPs had an effect on the toxicity of cobalt ferrite and titanium dioxide NPs, but not on zinc oxide NPs. This literature search also indicated that the present study is the only study so far that has assessed the effect of NP charge on the in vitro developmental toxicity of NPs, showing that positively charged PS-NPs are more potent than negatively charged PS-NPs. The review also reveals however that the effect of surface charge may vary with the type of NPs investigated, since the silicon dioxide, silver and titanium dioxide NPs that were reported to inhibit ES-D3 cell differentiation were negatively charged, while the negatively charged PS-NPs of the present study did not affect ES-D3 cell differentiation.

A comparison of effect concentrations on the inhibition of ES-D3 cell differentiation with effect concentrations on the decrease of ES-D3 cell viability reveals that for various NPs (cobalt ferrite NPs, gold NPs, silicon dioxide NPs and titanium dioxide NPs) ES-D3 cell differentiation is already inhibited at concentrations that do not yet affect cell viability, whereas for other NPs (PS-NPs, silver NPs and zinc oxide NPs), ES-D3 cell differentiation is only inhibited at concentrations that also affect cell viability. These differences between NPs may point to differences in modes of action underlying their inhibition of ES-D3 cell differentiation. In summary, the review shows that, in spite of the limited available data, smaller NPs may be more potent than larger NPs made of the same core material and that NP coating and surface charge can affect the NP-induced decrease of ES-D3 cell viability and inhibition of ES-D3 cell differentiation, albeit in an NP-type specific way. 


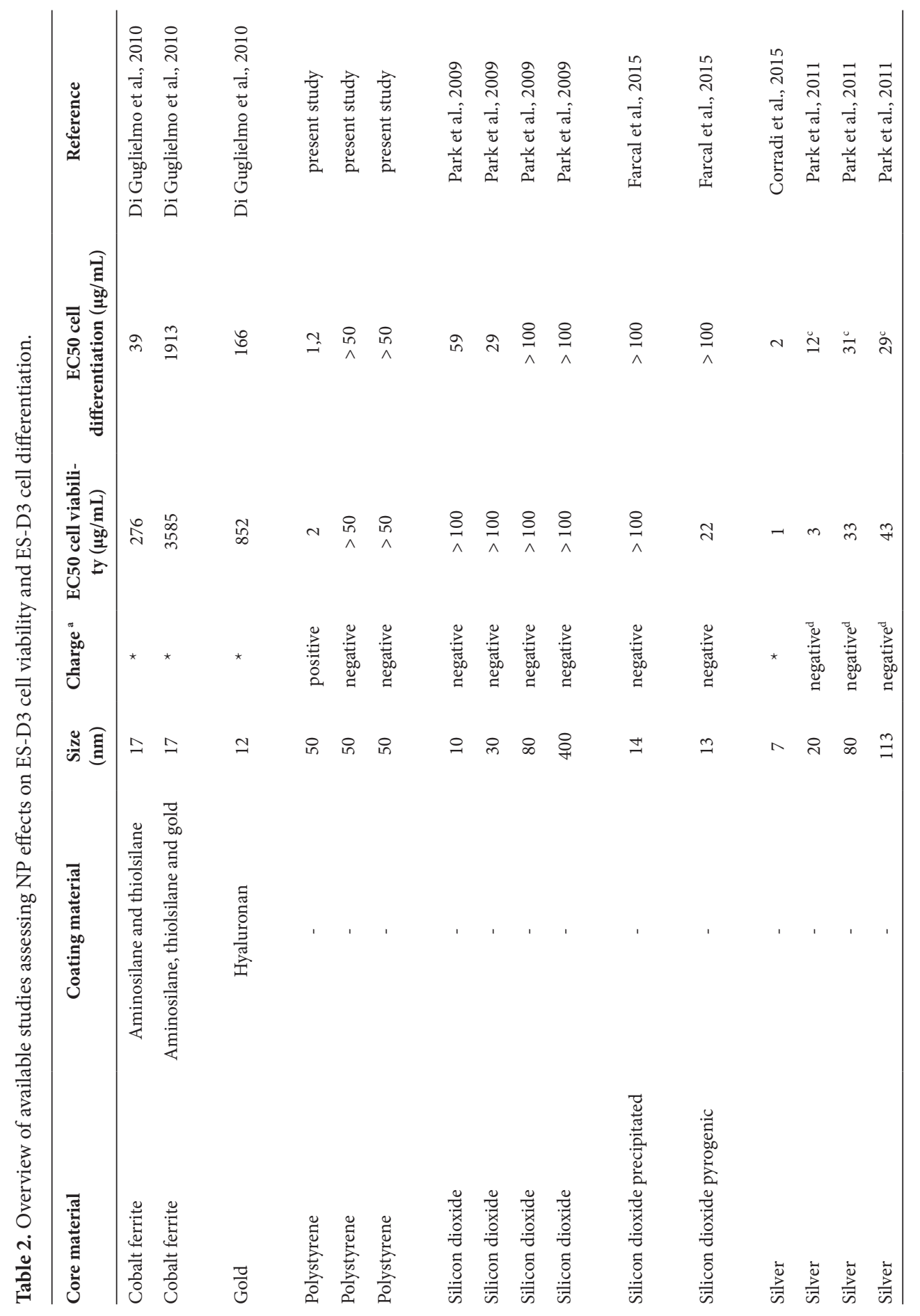




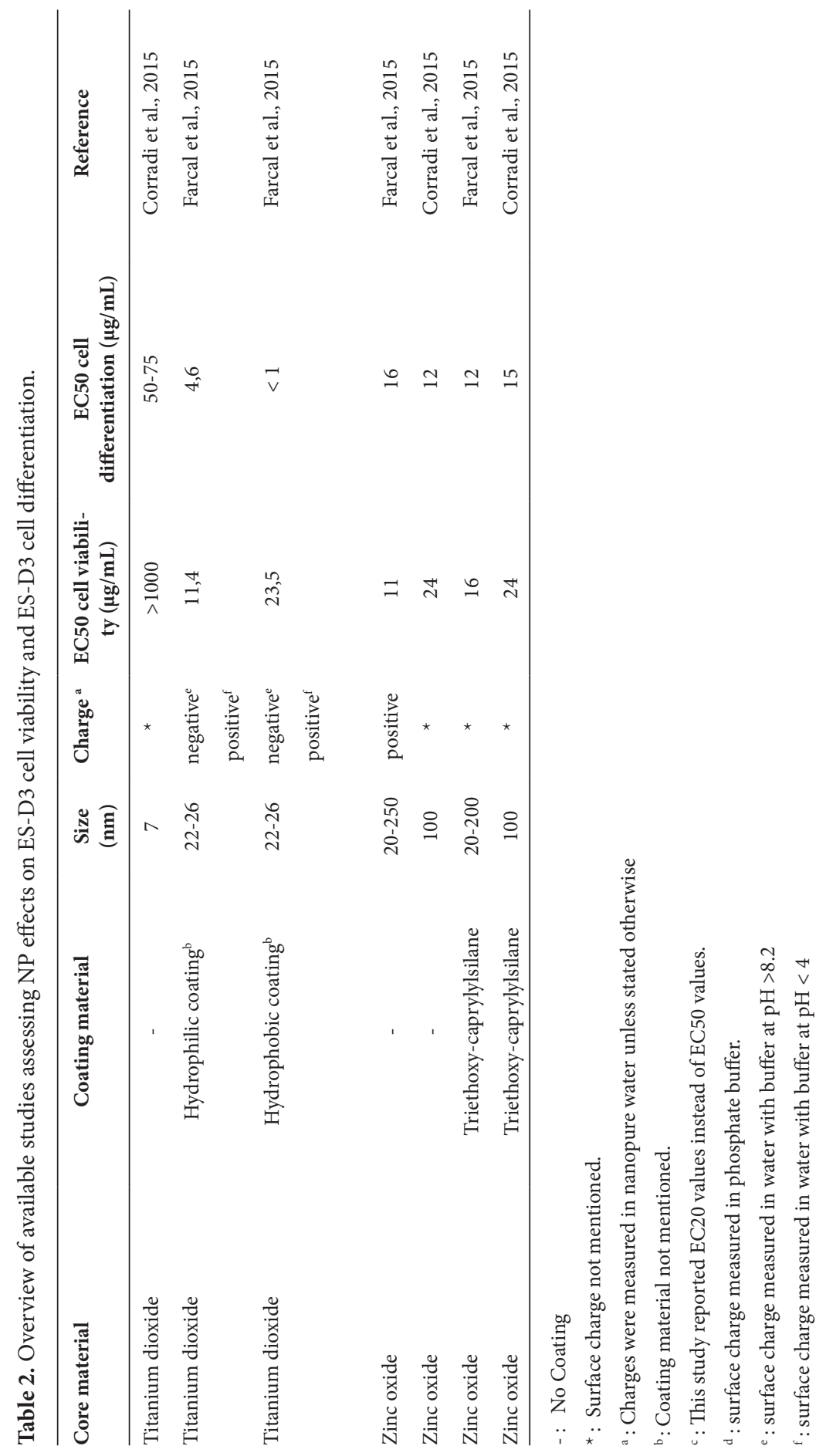




\section{DISCUSSION}

The present study aimed to assess the role of NP surface charge on the in vitro developmental toxicity potential of NPs as measured in the ES-D3 cell differentiation assay. The results show that the positively charged PS-NPs caused a concentration-dependent inhibition of ES-D3 cell differentiation while the two negatively charged PS-NPs did not show any effect, indicating that NP charge may play an important role in NP-induced developmental toxicity. In order to obtain insight whether also other physicochemical characteristics of NPs may relate to their in vitro developmental toxicity potency, we performed a literature search on the reported effects of NPs on ES-D3 cell differentiation. This analysis indicated that besides surface charge, also size and coating are important NP characteristics that may determine the developmental toxicity potential of NPs.

Effect concentrations of the positively charged PS-NPs in the ES-D3 cell differentiation assay were close to cytotoxic concentrations as measured in cell viability studies (Figure 1A), which indicates that the inhibition of the ES-D3 cell differentiation may be caused by non-specific effects. Similar effects have also been found for silver NPs and zinc oxide NPs (Table 2). In the validation study of the EST, most developmental toxicants that were selected based on positive developmental toxicity effects in in vivo studies appeared to cause inhibition of ES-D3 cell differentiation at concentrations that also caused cytotoxicity (Marx-Stoelting et al. 2009). Therefore, the positively charged PS-NPs tested in the present study may present a developmental hazard in vivo, even though the mode of action underlying this developmental toxicity may be related to cytotoxicity.

Knowledge on the relation between physicochemical characteristics of NPs, such as size or surface charge, and their toxicity would be of great help for setting priorities for NP toxicity testing. Although the experiments in the present study show that positively charged PS-NPs are more toxic than negatively charged PS-NPs, it appeared that this may not be generalizable to other NPs, following from the fact that the negatively charged PS-NPs of the present study did not affect ES-D3 cell differentiation, while silicon dioxide, silver and titanium dioxide NPs that were reported to inhibit ES-D3 cell differentiation were also negatively charged (Table 2). The reported surface charges of these NPs were measured in either nanopure water or phosphate buffer. This implies that the negative charge did not arise from serum proteins which may affect the NP charge, as observed in the present study when NP charge was measured in medium with FCS. To obtain better insight in the role of NP surface charge on developmental toxicity more studies are needed that assess the effects of NPs with different charge that are made of the same core material. The effect of surface charge on the potential to affect cell viability has been demonstrated before in other cell lines for tri-block copolymer NPs (Bhattacharjee et al. 2012), gold NPs (Liu et al. 2013) and silicon NPs (Bhattacharjee et al. 2013), 
generally showing higher cytotoxicity for positively charged NPs compared to negatively charged NPs. Whether this implies that positively charged silicon dioxide, silver and titanium dioxide NPs would show more potent effects on ES-D3 cell viability and differentiation than reported for their negative counterparts in literature (Table 2) remains to be investigated.

Our literature search showed that for two different types of NPs (silicon dioxide NPs and silver NPs), it has been shown that the effects on ES-D3 cell viability and differentiation were found at lower concentrations for relatively small NPs than for larger NPs of the same core material, indicating the role of NP size in their effects on ES-D3 cell viability and differentiation. The smaller the diameter of a spherical NP the higher the surface area which is often accompanied by increased chemical reactivity with cells (Nel et al. 2009). It has also been reported that cellular uptake of NPs is dependent on their size, showing a higher cellular uptake for relatively small NPs (Kreyling et al. 2009, Dos Santos et al. 2011), indicating that the intracellular concentration of small-sized NPs may be higher than the intracellular concentration of the larger sized NPs, which may also explain their higher toxic potency. However, when comparing toxic potencies of NPs of different sizes, it must be taken into account that when expressing exposure on a mass-basis $(\mu \mathrm{g} / \mathrm{ml})$ the number of NPs is larger for small-sized NPs than for large-sized NPs at the same concentration. This may need to be accounted for when comparing the toxicity potencies of NPs of different sizes.

Our analysis of the available literature also indicated that the in vitro developmental toxicity potency of NPs may also largely depend on the coating applied. However, from the available data it cannot be concluded whether the toxicity potential is dependent on the coating alone, or whether that is dependent on the combination of the core material and the coating. Therefore, although the limited data available indicate that charge, size and coating of NPs may be important characteristics that determine the developmental toxicity potential of NPs, more (systematic) studies are needed to assess how physicochemical characteristics of NPs relate to their developmental toxicity potency. This information may help to prioritize NPs for in vitro and in vivo developmental toxicity testing.

The results of the present study indicate that the positively charged PS-NPs may pose a hazard for embryonic development. However, to possibly become a risk for embryonic development, NPs need to be able to reach the developing embryo in the in vivo situation. Therefore, with oral exposure, NPs would need to translocate across the intestinal barrier as well as the placental barrier. The bioavailability upon oral exposure has been reported to be low for many NPs, because of a limited translocation across the intestinal barrier (Walczak et al. 2014). As far as we know, no in vivo study using oral exposure has shown so far that NPs can cause developmental toxicity. However, in vivo studies using intravenous injection of NPs have shown that NPs can cause adverse effects to the developing embryo, indicating that NPs can trans- 
locate across the placenta in the in vivo situation (Semmler-Behnke et al. 2008, Yamashita et al. 2011, Yang et al. 2012, Qi et al. 2014). In an earlier study, we have assessed the potential of the same PS-NPs as used in the present study to translocate across an in vitro barrier of human placental (BeWo) cells (Kloet et al. 2015). The results of these studies indicated that the positively charged PS-NPs hardly translocated across the BeWo cell layer, which may indicate that fetal exposure of these PS-NPs is low. Therefore, although the present study indicates the potential of positively charged PS-NPs to cause developmental toxicity, concentrations shown in the present study to be required to exert these effects may not be reached in the in vivo situation, because of the low placental transfer. Therefore, in order to predict which NPs may cause developmental toxicity in the in vivo situation, data obtained in the in vitro ES-D3 cell differentiation assay should be combined with placental translocation data from the BeWo model in order to select the NPs that are expected to have the highest potency in vivo. These combined data may then be used to select and prioritize NPs that should be assessed in vivo for potential developmental toxicity.

\section{ACKNOWLEDGEMENTS}

This work is supported by NanoNextNL, a micro and nanotechnology consortium of the Government of the Netherlands and 130 partners. 


\section{REFERENCES}

Bhattacharjee, S., D. Ershov, K. Fytianos, J. Gucht, G. Alink, I. Rietjens, A. Marcelis and H. Zuilhof (2012). "Cytotoxicity and cellular uptake of tri-block copolymer nanoparticles with different size and surface characteristics." Particle and Fibre Toxicology 9(1): 11.

Bhattacharjee, S., I. M. Rietjens, M. P. Singh, T. M. Atkins, T. K. Purkait, Z. Xu, S. Regli, A. Shukaliak, R. J. Clark, B. S. Mitchell, G. M. Alink, A. T. Marcelis, M. J. Fink, J. G. Veinot, S. M. Kauzlarich and H. Zuilhof (2013). "Cytotoxicity of surface-functionalized silicon and germanium nanoparticles: the dominant role of surface charges." Nanoscale 5(11): 4870-4883.

Braakhuis, H. M., S. K. Kloet, S. Kezic, F. Kuper, M. V. Park, S. Bellmann, M. van der Zande, S. Le Gac, P. Krystek, R. J. Peters, I. M. Rietjens and H. Bouwmeester (2015). "Progress and future of in vitro models to study translocation of nanoparticles.” Arch Toxicol 89(9): 1469-1495.

Campagnolo, L., I. Fenoglio, M. Massimiani, A. Magrini and A. Pietroiusti (2013). "Screening of nanoparticle embryotoxicity using embryonic stem cells." Methods Mol Biol 1058: 49-60.

de Jong, E., M. Barenys, S. A. B. Hermsen, A. Verhoef, B. C. Ossendorp, J. G. M. Bessems and A. H. Piersma (2011). "Comparison of the mouse Embryonic Stem cell Test, the rat Whole Embryo Culture and the Zebrafish Embryotoxicity Test as alternative methods for developmental toxicity testing of six 1,2,4-triazoles." Toxicology and Applied Pharmacology 253(2): 103-111.

de Jong, E., J. Louisse, M. Verwei, B. J. Blaauboer, J. J. van de Sandt, R. A. Woutersen, I. M. Rietjens and A. H. Piersma (2009). "Relative developmental toxicity of glycol ether alkoxy acid metabolites in the embryonic stem cell test as compared with the in vivo potency of their parent compounds." Toxicol Sci 110(1): 117-124.

Dos Santos, T., J. Varela, I. Lynch, A. Salvati and K. A. Dawson (2011). "Quantitative Assessment of the Comparative Nanoparticle-Uptake Efficiency of a Range of Cell Lines." Small.

Fort, D. J. and R. R. Paul (2002). "Enhancing the predictive validity of Frog Embryo Teratogenesis Assay--Xenopus (FETAX).” J Appl Toxicol 22(3): 185-191.

Gebel, T., R. Marchan and J. G. Hengstler (2013). “The nanotoxicology revolution.” Arch Toxicol 87(12): 2057-2062.

Genschow, E., H. Spielmann, G. Scholz, I. Pohl, A. Seiler, N. Clemann, S. Bremer and K. Becker (2004). "Validation of the embryonic stem cell test in the international ECVAM validation study on three in vitro embryotoxicity tests." Altern Lab Anim 32(3): 209-244.

Jelinek, R., M. Peterka and Z. Rychter (1985). “Chick embryotoxicity screening test--130 substances tested.” Indian J Exp Biol 23(10): 588-595.

Kloet, S. K., A. P. Walczak, J. Louisse, H. H. van den Berg, H. Bouwmeester, P. Tromp, R. G. Fokkink and I. M. Rietjens (2015). "Translocation of positively and negatively charged polystyrene nanoparticles in an in vitro placental model.” Toxicol In Vitro 29(7): 1701-1710.

Kreyling, W. G., M. Semmler-Behnke, J. Seitz, W. Scymczak, A. Wenk, P. Mayer, S. Takenaka and G. Oberdorster (2009). "Size dependence of the translocation of inhaled iridium and carbon nanoparticle aggregates from the lung of rats to the blood and secondary target organs." Inhal Toxicol 21 Suppl 1: 55-60.

Li, H., I. M. Rietjens, J. Louisse, M. Blok, X. Wang, L. Snijders and B. van Ravenzwaay (2015). "Use of the ES-D3 cell differentiation assay, combined with the BeWo transport model, to predict relative in vivo developmental toxicity of antifungal compounds." Toxicol In Vitro 29(2): 320-328.

Liu, X., N. Huang, H. Li, Q. Jin and J. Ji (2013). "Surface and Size Effects on Cell Interaction of Gold Nanoparticles with Both Phagocytic and Nonphagocytic Cells.” Langmuir 29(29): 9138-9148.

Marx-Stoelting, P., E. Adriaens, H. J. Ahr, S. Bremer, B. Garthoff, H. P. Gelbke, A. Piersma, C. Pellizzer, U. Reuter, V. Rogiers, B. Schenk, S. Schwengberg, A. Seiler, H. Spielmann, M. Steemans, D. B. Stedman, P. Vanparys, J. A. Vericat, M. Verwei, F. van der Water, M. Weimer and M. Schwarz (2009). "A review of the implementation of the embryonic stem cell test (EST). The report and recommendations of an ECVAM/ReProTect Workshop." Altern Lab Anim 37(3): 313-328.

Nel, A. E., L. Madler, D. Velegol, T. Xia, E. M. Hoek, P. Somasundaran, F. Klaessig, V. Castranova and M. Thompson (2009). "Understanding biophysicochemical interactions at the nano-bio interface." Nat Mater 8(7): 543557.

Oberdorster, G., V. Stone and K. Donaldson (2007). “Toxicology of nanoparticles: A historical perspective." Nanotoxicology 1(1): 2-25. 
Park, M. V., W. Annema, A. Salvati, A. Lesniak, A. Elsaesser, C. Barnes, G. McKerr, C. V. Howard, I. Lynch, K. A. Dawson, A. H. Piersma and W. H. de Jong (2009). "In vitro developmental toxicity test detects inhibition of stem cell differentiation by silica nanoparticles." Toxicol Appl Pharmacol 240(1): 108-116.

Pietroiusti, A., M. Massimiani, I. Fenoglio, M. Colonna, F. Valentini, G. Palleschi, A. Camaioni, A. Magrini, G. Siracusa, A. Bergamaschi, A. Sgambato and L. Campagnolo (2011). "Low Doses of Pristine and Oxidized Single-Wall Carbon Nanotubes Affect Mammalian Embryonic Development.” ACS Nano 5(6): 4624-4633.

Qi, W., J. Bi, X. Zhang, J. Wang, J. Wang, P. Liu, Z. Li and W. Wu (2014). "Damaging effects of multi-walled carbon nanotubes on pregnant mice with different pregnancy times.” Sci Rep 4: 4352.

Semmler-Behnke, M., W. G. Kreyling, J. Lipka, S. Fertsch, A. Wenk, S. Takenaka, G. Schmid and W. Brandau (2008). "Biodistribution of 1.4- and 18-nm gold particles in rats." Small 4(12): 2108-2111.

Spielmann, H., A. Seiler, S. Bremer, L. Hareng, T. Hartung, H. Ahr, E. Faustman, U. Haas, G. J. Moffat, H. Nau, P. Vanparys, A. Piersma, J. R. Sintes and J. Stuart (2006). “The practical application of three validated in vitro embryotoxicity tests. The report and recommendations of an ECVAM/ZEBET workshop (ECVAM workshop 57)." Altern Lab Anim 34(5): 527-538.

van Dartel, D. A., J. L. Pennings, L. J. de la Fonteyne, K. J. Brauers, S. Claessen, J. H. van Delft, J. C. Kleinjans and A. H. Piersma (2011). "Concentration-dependent gene expression responses to flusilazole in embryonic stem cell differentiation cultures." Toxicol Appl Pharmacol 251(2): 110-118.

van Dartel, D. A., J. L. Pennings, P. J. Hendriksen, F. J. van Schooten and A. H. Piersma (2009). “Early gene expression changes during embryonic stem cell differentiation into cardiomyocytes and their modulation by monobutyl phthalate." Reprod Toxicol 27(2): 93-102.

van Dartel, D. A. M., N. J. L. Zeijen, L. J. J. de la Fonteyne, F. J. van Schooten and A. H. Piersma (2009). “Disentangling cellular proliferation and differentiation in the embryonic stem cell test, and its impact on the experimental protocol." Reproductive Toxicology 28(2): 254-261.

Walczak, A. P., E. Kramer, P. J. Hendriksen, P. Tromp, J. P. Helsper, M. van der Zande, I. M. Rietjens and H. Bouwmeester (2014). "Translocation of differently sized and charged polystyrene nanoparticles in in vitro intestinal cell models of increasing complexity." Nanotoxicology: 1-9.

Yamashita, K., Y. Yoshioka, K. Higashisaka, K. Mimura, Y. Morishita, M. Nozaki, T. Yoshida, T. Ogura, H. Nabeshi, K. Nagano, Y. Abe, H. Kamada, Y. Monobe, T. Imazawa, H. Aoshima, K. Shishido, Y. Kawai, T. Mayumi, S. Tsunoda, N. Itoh, T. Yoshikawa, I. Yanagihara, S. Saito and Y. Tsutsumi (2011). "Silica and titanium dioxide nanoparticles cause pregnancy complications in mice." Nat Nanotechnol 6(5): 321-328.

Yang, H., C. Sun, Z. Fan, X. Tian, L. Yan, L. Du, Y. Liu, C. Chen, X. J. Liang, G. J. Anderson, J. A. Keelan, Y. Zhao and G. Nie (2012). "Effects of gestational age and surface modification on materno-fetal transfer of nanoparticles in murine pregnancy." Sci Rep 2: 847.

zur Nieden, N. I., G. Kempka and H. J. Ahr (2004). "Molecular multiple endpoint embryonic stem cell test--a possible approach to test for the teratogenic potential of compounds." Toxicol Appl Pharmacol 194(3): 257-269. 


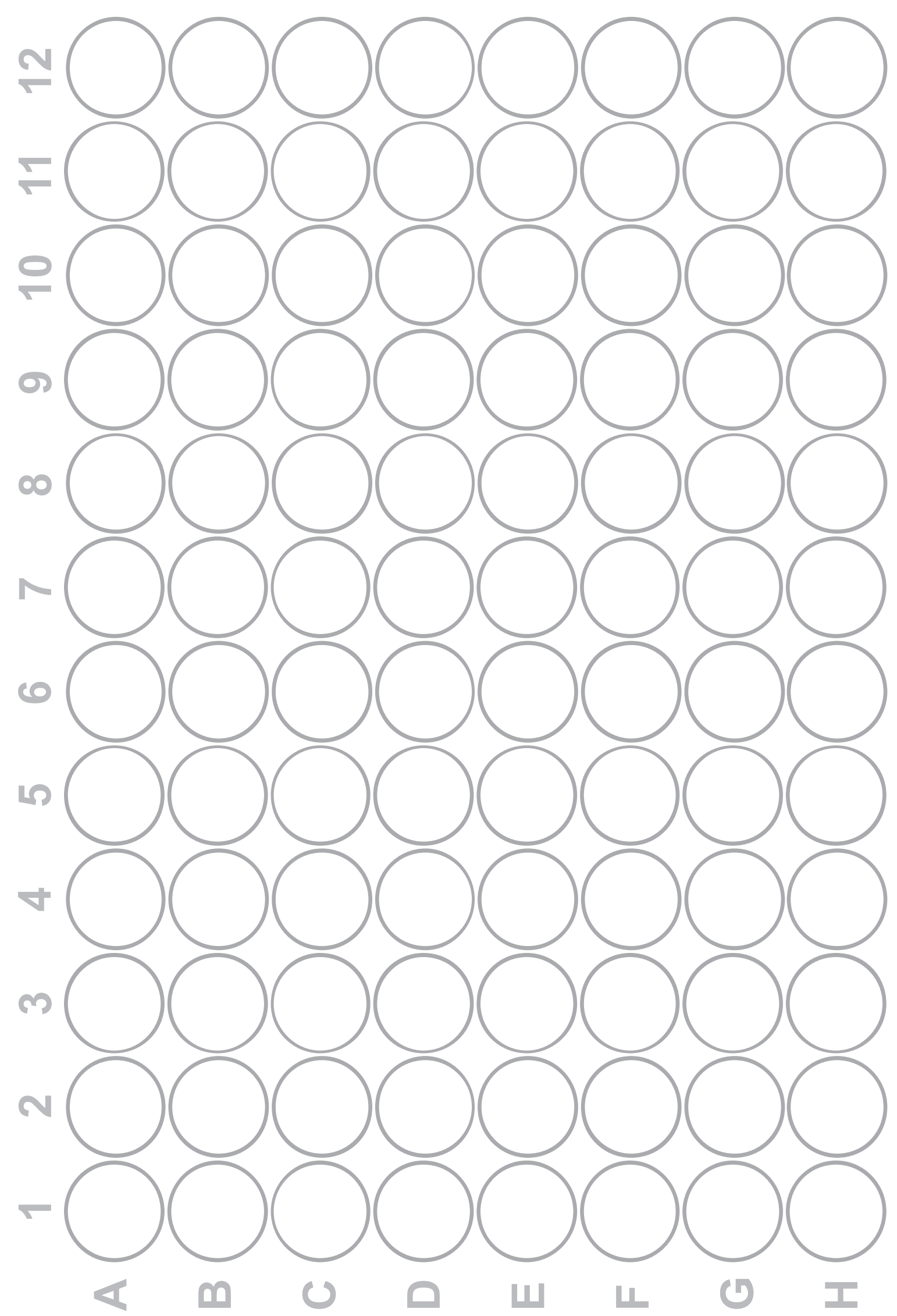




\section{Chapter 5}

\section{Effects of nanoparticle forms of metal (oxide) food additives and related nanoparticles on cell viability, TNF- $\alpha$ production and mitochondria-related parameters in RAW264.7 macrophages}

Samantha K. Kloet

Sunday Makama

Hans H.J. van den Berg

Gang Wu

Georgios Saviolakis

Heike Vogt

Aikaterini Manolesou

Nico van den Brink Ivonne M.C.M. Rietjens

Jochem Louisse 


\section{ABSTRACT}

Specifications of food additives like titanium dioxide, iron oxide, silicon dioxide and silver do not exclude the material of commerce being present in part in nanoform. The aim of the present study was to investigate toxic effects of the nanoform of these metal (oxide) food additives and other nanoparticles (NPs), being manganese oxide, copper oxide, chromium oxide, cobalt oxide and nickel oxide NPs, using an in vitro model of macrophage RAW264.7 cells. Effects on cell viability, reactive oxygen species (ROS) production, mitochondrial permeability transition pore (MPTP) opening, ATP, and TNF- $\alpha$ were assessed. The results indicate that most of the tested NPs show no effects on the selected endpoints up to the highest concentration tested. Of the food additive related NPs, silicon dioxide and silver NPs were most toxic, showing a decrease in cell viability at concentrations of $100 \mu \mathrm{g} / \mathrm{ml}$ and higher. Altogether, the present study shows no or limited effects of the NP forms of metal (oxide) food additives on cell viability, ROS production, MPTP opening, ATP levels and TNF- $a$ production in RAW264.7 macrophages. Effects were only observed at high concentrations that may not be physiologically relevant, indicating that related adverse effects upon exposure to the respective NPs may be limited. 


\section{INTRODUCTION}

Several food additives, such as titanium dioxide $\left(\mathrm{TiO}_{2}\right)(\mathrm{E} 171)$, iron (III) oxide $\left(\mathrm{Fe}_{2} \mathrm{O}_{3}\right)(\mathrm{E} 172)$, silicon dioxide $\left(\mathrm{SiO}_{2}\right)(\mathrm{E} 551)$ and silver (Ag) (E174), contain metal or metal oxide particles of variable sizes (Athinarayanan et al. 2014, Periasamy et al. 2015). At present the specifications of these food additives do not exclude the presence of the compounds in their nanoform, because food additives are excluded from the regulation that states that all ingredients that are present in nano-form should be indicated in the list of ingredients as 'nano' (2011). A recent study investigated the morphology and dimensions of the food additive $\mathrm{SiO}_{2}$ in several commercial food products, showing the presence of spherical $\mathrm{SiO}_{2}$ nanoparticles (NPs) of about 10-50 $\mathrm{nm}$ in size (Athinarayanan et al. 2014). Other studies found that a certain food grade $\mathrm{TiO}_{2}$ contained $\sim 25-38 \%$ of the $\mathrm{TiO}_{2}$ as NPs smaller than $200 \mathrm{~nm}$ (Weir et al. 2012, Faust et al. 2014). These findings indicate that studies on the health effects of nanoforms of current food additives are of high interest. At the same time, safety considerations for nanoscale materials in food products have not been well developed yet (Bouwmeester et al. 2009). As the health effects of NPs are largely unknown, toxicity studies with nanomaterials that measure such health effects are urgently needed.

The aim of the present study was to investigate the toxic effects of the nanoform of metal (oxide) food additives $\mathrm{TiO}_{2}, \mathrm{Fe}_{2} \mathrm{O}_{3}, \mathrm{SiO}_{2}$ and $\mathrm{Ag}$ using an in vitro model consisting of macrophage RAW264.7 cells. Further, the effects of other metal oxide NPs of manganese oxide $\left(\mathrm{Mn}_{2} \mathrm{O}_{3}\right)$, copper oxide $(\mathrm{CuO})$, chromium oxide $\left(\mathrm{Cr}_{2} \mathrm{O}_{3}\right)$, cobalt oxide $\left(\mathrm{Co}_{3} \mathrm{O}_{4}\right)$ and nickel oxide $(\mathrm{NiO})$, to which we may be exposed via products like paints, catalysts, construction materials, coatings and batteries were quantified (Siddiqui et al. 2012, Tavares et al. 2014, Dang et al. 2015). The possible NP-induced adverse effects on macrophage RAW264.7 cells may indicate possible immunotoxic responses and hazards since macrophages play a major role in the innate immune response, providing a first line of defence (Ma et al. 2003, Elsabahy et al. 2013). NP-induced macrophage cell toxicity may therefore decrease the innate immune response. Also, activation of cytokine production, such as tumor necrosis factor- $\alpha$ (TNF- $\alpha$ ), by macrophages exposed to NPs is undesired, since this may lead to inflammatory reactions.

To assess effects of the NPs on the macrophages, cell viability, mitochondria-related parameters and release of the inflammatory mediator TNF- $\alpha$ were assessed in NP-exposed RAW264.7 cells. This study focussed on the effects of the NPs on mitochondria-related parameters, since previous research indicated that mitochondria play an important role in NP-induced toxicity (Park et al. 2008, Sharma et al. 2011, Shvedova et al. 2012, Bhattacharjee et al. 2013). Especially cationic NPs have been reported to damage the mitochondria, leading to disturbance of intracellular adenosine $5^{\prime}$-triphosphate (ATP) and reactive oxygen species (ROS) production, which may trigger apoptotic reactions that ultimately lead to the observed toxicity (Bhat- 
tacharjee et al. 2013). Therefore, in the present study the effects of the metal oxide NPs on ROS production, mitochondrial permeability transition pore (MPTP) opening and intracellular ATP levels were assessed, in addition to effects on cell viability and production of TNF- $\alpha$.

\section{MATERIALS AND METHODS}

\section{Materials}

High glucose Dulbecco's Modified Eagle Medium (DMEM/F-12), Phosphate Buffered Saline (PBS), Fetal Calf Serum (FCS), Hank's Balanced Salt Solution (HBSS) and trypsin-EDTA were obtained from Invitrogen (Breda, the Netherlands). Bovine Serum Albumin (BSA), 3-(4,5-Dimethylthiazol-2-yl)-2,5-diphenyltetrazolium bromide (MTT) and copper sulfate were purchased from Sigma Aldrich (Zwijndrecht, the Netherlands). WST-1 Cell Proliferation Reagent was purchased from Roche (Woerden, The Netherlands). Information on size and specific surface area of the NPs that were used in the present study as provided by the manufacturers are presented in table $1 . \mathrm{Fe}_{2} \mathrm{O}_{3} \mathrm{NPs}, \mathrm{TiO}_{2}$ anatase and $\mathrm{TiO}_{2}$ rutile NPs, and $\mathrm{Cr}_{2} \mathrm{O}_{3}$ NPs were obtained from US Research Nanomaterials (Houston, Texas, USA). $\mathrm{Mn}_{2} \mathrm{O}_{3}$ NPs were obtained from Auer Remy (Hamburg, Germany). $\mathrm{SiO}_{2} \mathrm{NPs}$ and NiO NPs were obtained from Skyspring (Houston, Texas, USA). $\mathrm{Co}_{3} \mathrm{O}_{4} \mathrm{NPs}$ and $\mathrm{CuO}$ NPs were obtained from Sigma Aldrich (Zwijndrecht, The Netherlands). Ag NPs were obtained from NanoComposix Inc. (San Diego, California, USA).

\section{Preparation of NPs for in vitro studies}

All NPs were delivered in powdered form except for Ag NPs which came as a stable $5 \mathrm{mg} / \mathrm{ml}$ aqueous suspension in nanopure water. The working suspensions were prepared according to a method described by Zhang et al. (2012). Briefly, a $5 \mathrm{mg} / \mathrm{ml}$ stock suspension in nanopure water of each powdered NP was prepared in a sterile glass vial. These suspensions were sonicated in a bath sonicator (VWR international, Leuven, Belgium) for 20 minutes. Afterwards, an equal volume of $4 \%$ BSA in nanopure water was added to stabilise the suspension. These suspensions were left for one hour at room temperature to equilibrate. After vortexing the NP stock suspension, further dilutions were prepared with 2\% BSA in nanopure water, except for the Ag NP stock suspension which was further diluted in nanopure water. Finally, the BSA-coated NP suspensions were $12.5 \mathrm{x}$ diluted in cell culture medium containing $10 \%$ FCS, reaching the final exposure NP concentrations between 0 and $200 \mu \mathrm{g} / \mathrm{ml}$.

\section{NP characterization}

The size (hydrodynamic diameter) of the NPs was determined by dynamic light scattering (DLS) using a Cobolt Samba 300mW DPSS laser at a wavelength of $532 \mathrm{~nm}$. Measurements were performed on freshly prepared NP suspensions at $10 \mu \mathrm{g} / \mathrm{ml}$ in nanopure water and in cell culture medium containing $10 \%$ FCS. For each sample, twenty measurements were made 
Table 1. Commercial source information for metal (oxide) NPs used in the present study. Reported size was determined by TEM and reported specific surface area was calculated using bulk density.

\begin{tabular}{llccc}
\hline $\mathbf{N P}$ & Source & Catalogue number & Size (nm) & $\begin{array}{c}\text { Specific surface } \\
\text { area (SSA) } \\
\left(\mathbf{m}^{2} / \mathbf{g}\right)\end{array}$ \\
\hline $\mathrm{TiO}_{2}$ Anatase & US Research Nanomaterials, Inc & US3498 & 50 \\
$\mathrm{TiO}_{2}$ Rutile & US Research Nanomaterials, Inc & US3520 & 30 & $35-60$ \\
$\mathrm{Fe}_{2} \mathrm{O}_{3}$ & US Research Nanomaterials, Inc & US3210 & $20-40$ & $40-60$ \\
$\mathrm{SiO}_{2}$ & Skyspring Nanomaterials, Inc & $6810 \mathrm{DL}$ & $20-60$ & $400-600$ \\
$\mathrm{Ag}$ & Nanocomposix & DAC1612 & 50 & 9.8 \\
$\mathrm{Mn}_{2} \mathrm{O}_{3}$ & Auer Remy & $3610 \mathrm{FY}$ & 30 & 14 \\
$\mathrm{Cr}_{2} \mathrm{O}_{3}$ & US Research Nanomaterials, Inc & US3060 & 60 & 20 \\
$\mathrm{CuO}$ & Sigma Aldrich & 544868 & $<50$ & $40-70$ \\
$\mathrm{Co}_{3} \mathrm{O}_{4}$ & Sigma Aldrich & 637025 & $<50$ & $>6$ \\
$\mathrm{NiO}$ & Skyspring Nanomaterials, Inc & $5410 S \mathrm{C}$ & 50 & 5 \\
\hline
\end{tabular}

and the detector intensity data (measured with an ALV/SO SIPD Single Photon Detector with ALV Static and Dynamic Fiber optics) were processed with an ALV5000/60X0 external correlator and ALV-5000/E software (all from ALV-GmbH, Germany). Data were analysed with the AfterALV software and mean diameter and polydispersity index (PDI) were determined for each NP sample. Additionally the zeta potential of the NPs was measured at a concentration of $100 \mu \mathrm{g} / \mathrm{ml}$ in both nanopure water and in cell culture medium containing $10 \%$ FCS using the Malvern Zetasizer 2000 (Malvern Instruments, UK).

Morphology of the NPs was characterised by scanning electron microscopy (SEM). To this end, suspensions of the NPs $(10-100 \mu \mathrm{g} / \mathrm{mL})$ in nanopure water were studied using SEM. Of each suspension $25 \mu \mathrm{l}$ was deposited and left to dry on a nickel-coated polycarbonate filter, which was mounted on an aluminium specimen holder. The high resolution field emission gun scanning electron microscope (FEG-SEM) used in this study was a Tescan MIRA-LMH FEG-SEM (Tescan, Brno, Czech Republic). The microscope was operated at an accelerating voltage of $15 \mathrm{kV}$, working distance (WD) of $10 \mathrm{~mm}$ and spot size of $5 \mathrm{~nm}$.

Images of cells exposed to $200 \mu \mathrm{g} / \mathrm{ml}$ were captured with an inverted fluorescence microscope (Olympux IX70, USA) equipped with a digital camera (Canon EOS 1000D, Japan). 


\section{Cell culture and assessment of cell viability}

RAW264.7 cells (LGC, ATCC, Teddington, UK) were cultured at $37^{\circ} \mathrm{C}$ with $5 \% \mathrm{CO}_{2}$ in a humidified atmosphere in $10 \mathrm{ml}$ DMEM supplemented with $10 \%(\mathrm{v} / \mathrm{v})$ FCS in a $75 \mathrm{~cm}^{2}$ culture flask (Corning, USA). Cells were subcultured every 3 to 4 days when reaching $80 \%$ confluency using trypsin-EDTA after rinsing with PBS.

To assess effects of the NPs on cell viability, RAW264.7 cells were seeded at $2 \times 10^{4}$ cells/well in $100 \mu \mathrm{l}$ culture medium with 10\% FCS in 96-well plates (Greiner bio-one) and incubated at $37^{\circ} \mathrm{C}$ and $5 \% \mathrm{CO}_{2}$ for 24 hours. After this, the medium was removed and cells were subsequently exposed to $100 \mu \mathrm{l}$ of NP suspensions using concentrations ranging from 0 to 200 $\mu \mathrm{g} / \mathrm{ml}$ in culture medium with $10 \%$ FCS. After 24 hours exposure, $10 \mu \mathrm{l}$ of WST solution was added to the medium. After 45 min incubation, the 96-well plate was shaken for 5 minutes (protected from light) and subsequently absorbance was measured at 450 and $620 \mathrm{~nm}$ (background) using a SpectraMax M2 Microplate Reader (Molecular Devices LLC, Sunnyvale, CA USA). For each experimental condition, formazan formation was expressed as percentage of the formazan formation of the solvent control. Ag NPs showed a concentration-dependent interference in this WST assay. Therefore, instead of WST solution, $5 \mu \mathrm{l}$ of a $5 \mathrm{mg} / \mathrm{mL}$ tetrazolium salt 3-(4,5-dimethylthiazol-2-yl)-2,5-diphenyltetrazolium bromide (MTT) solution in PBS was added to each well in the experiments on Ag NPs and incubated for 1.5 hours. The exposure media containing MTT was then removed and $100 \mu \mathrm{L}$ DMSO was added to solubilize the formazan crystals, which was achieved by shaking the plates protected from light for $10 \mathrm{~min}$. The dissolved formazan was quantified by measuring the absorption at $562 \mathrm{~nm}$ and $620 \mathrm{~nm}$ (background) using a SpectraMax M2 Microplate Reader. For each experimental condition, formazan formation was expressed as percentage of the formazan formation of the solvent control. Copper sulfate $(100 \mu \mathrm{M})$ was used as the positive control.

\section{Assessment of mitochondrial permeability transition pore (MPTP) opening}

The MPTP opening refers to a change in the inner mitochondrial membrane permeability (Bonora et al. 2014). An increase in MPTP opening results in an increase in the mitochondrial membrane permeability, leading to rapid loss of the mitochondrial membrane potential, a decrease in ATP production, and finally to the loss of cell integrity (Ly et al. 2003). After 24 hours exposure to the NPs under similar conditions as with the cell viability assay, MPTP opening was measured using the MitoProbe Transition Pore Assay Kit (Invitrogen, Breda, The Netherlands, cat nr. M34153). Fluorescence was measured at 485 and $530 \mathrm{~nm}$ (background) using a SpectraMax M2 Microplate Reader. Ionomycin, which was supplied with the kit, was used as the positive control ( 15 minutes incubation at a concentration of $10 \mu \mathrm{M})$. In the assay, an increase in MPTP opening causes a decrease in the fluorescent signal which correlates with an increase in the mitochondrial membrane permeability, so with a decrease in the mitochondrial membrane integrity. 


\section{Assessment of intracellular ROS production}

To determine the effects of the NPs on intracellular ROS production, RAW264.7 cells were seeded at $2 \times 10^{4}$ cells/well in $100 \mu \mathrm{l}$ culture medium with $10 \%$ FCS in 96-well plates and incubated at $37^{\circ} \mathrm{C}$ and $5 \% \mathrm{CO}_{2}$ for 24 hours, followed by removal of the medium and addition of a solution of $20 \mu \mathrm{M}$ 2,7-dichlorodihydrofluorescein diacetate (DCFDA) in medium with $10 \%$ FCS. After 45 minutes the DCFDA solution was removed and cells were exposed for 24 hours to the NPs under similar conditions as with the cell viability assay. Fluorescence was measured using a SpectraMax M2 Microplate Reader at 485 and $538 \mathrm{~nm}$ (background) wavelengths. $\mathrm{H}_{2} \mathrm{O}_{2}(10 \mathrm{mM})$ was used as the positive control.

\section{Assessment of intracellular ATP levels}

The effects of NPs on intracellular ATP levels was determined. After 24 hours exposure to the NPs under similar conditions as with the cell viability assay, the ATP levels were determined using the ATP bioluminescent somatic cell assay kit (Sigma Aldrich, Zwijndrecht, The Netherlands, cat nr. FLASC) by measuring luminescence using the Luminoscan Ascent (Thermo electroncorporation). 2,4-Dinitrophenol (4 mM) was used as the positive control.

\section{Assessment of TNF- $\alpha$ production}

To determine the effects of the NPs on TNF- $\alpha$ production, the sandwich ELISA mouse TNF- $\alpha$ kit (Invitrogen, Breda, The Netherlands, cat nr. KMC3011C) was used. After 24 hours exposure to the NPs under similar conditions as with the cell viability assay, plates were centrifuged (centrifuge model $4 \mathrm{k} 10$ from Sigma) at $1200 \mathrm{rpm}$ for 5 minutes at $4^{\circ} \mathrm{C}$. Subsequently, the supernatants were collected and frozen at $-80^{\circ} \mathrm{C}$. Samples were thawed and added to microtiter strips, coated with monoclonal antibodies specific for mouse TNF- $\alpha$. The samples were diluted twice with standard diluent buffer from the kit and the secondary antibody (biotinylated mouse TNF- $\alpha$ Biotin Conjugate) was added. After 90 minutes of incubation, the wells were washed four times with wash buffer (to remove any unbound secondary antibody) after which a $100 \mu \mathrm{l}$ solution containing Streptavidin-HRP was added, which binds to the biotinylated secondary antibody. The plate was covered with a plastic cover and stored for 30 minutes at room temperature and the wells were subsequently washed four times with wash buffer delivered with the kit. Stabilized chromogen was added to each well and the plate was stored for 20 to 25 minutes in the dark. Subsequently, a stop solution was added to each well and the absorbance of each well was read at $450 \mathrm{~nm}$, using a SpectraMax M2 Microplate Reader. A series of TNF- $\alpha$ concentrations were included in duplicate to each 96 wells plate in order to define a calibration curve enabling quantification of TNF- $\alpha$. Lipopolysaccharide (LPS, $0.1 \mu \mathrm{g} / \mathrm{ml}$ ) was used as the positive control. 


\section{Data analysis}

For each study, three or four independent experiments were performed $(n=3-4)$. In each experiment three technical replicates were included, which were averaged per experiment. Data are shown as mean \pm standard deviation (SD) of all data points per experiment and plotted in graphs using GraphPad Prism version 5. Statistical analyses among mean values of the different treatments were performed by one-way analysis of variance followed by a Tukey's Multiple Comparison Test using GraphPad Prism version 5.

\section{RESULTS}

\section{Physicochemical characterization of the NPs}

NP size, zeta potential and morphology were characterized using SEM, DLS and zeta potential analyses. Figure 1 shows the SEM pictures of the different NPs. Ag NPs are rather spherical shaped and monodispersed, while the other NPs have variable shapes and form agglomerates (Figure 1).

Dynamic light scattering (DLS) measurements were performed to determine the hydrodynamic diameter of the different NPs in nanopure water and cell culture medium with $10 \%$ FCS (Table 2). All NPs agglomerated when dispersed in water or cell culture medium with

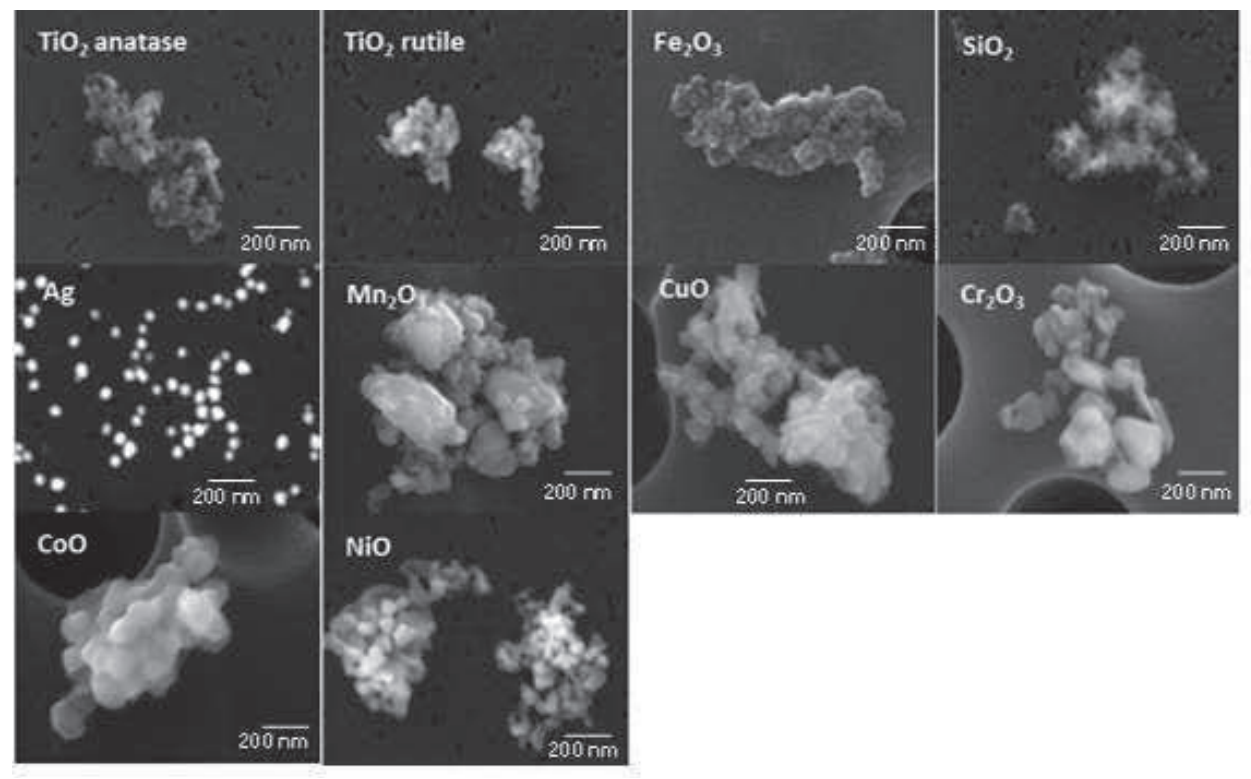

Figure 1. SEM pictures (magnification $200000 x$ ) of the NPs used in the present study. 
10\% FCS albeit to varying degrees. For all NPs, except Ag NPs, their hydrodynamic size was higher in water compared to cell culture medium, indicating more agglomeration in water (Table 2). Of all NPs used in the present study only Ag NPs had a hydrodynamic size close to the size as given by the manufacturer (Table 1). The PDI of the NPs varied between 0.2 and 0.6. For all NPs except $\mathrm{Mn}_{2} \mathrm{O}_{3}$, the PDI was higher for cell culture medium as compared to the PDI in water, indicating that the size distribution became wider. Background values measured for cell culture medium with 10\% FCS and water are 12.1 and $0 \mathrm{~nm}$ respectively.

The zeta potential of the NPs was measured as an indication of the charge of the NPs in both water and cell culture medium with 10\% FCS (Table 2). All NPs had a zeta potential in water between -29.6 and $-46.1 \mathrm{mV}$. In cell culture medium the zeta potential of all NPs varied between -8.9 and $-17.1 \mathrm{mV}$. Background values measured for cell culture medium with $10 \%$ FCS and water are -6.4 and $3.3 \mathrm{mV}$ respectively.

Table 2. Dynamic light scattering (DLS) $(n=3)$ and zeta potential $(n=3)$ results.

\begin{tabular}{lcccc}
\hline $\mathbf{N P}$ & \multicolumn{2}{c}{ Diameter in $\mathbf{n m} \pm$ SD (PDI) } & \multicolumn{2}{c}{ Zeta potential (mV) \pm SD } \\
& In water & $\begin{array}{c}\text { In cell culture medium } \\
\text { with } 10 \% \mathrm{FCS}\end{array}$ & In water & $\begin{array}{c}\text { In cell culture medium } \\
\text { with } 10 \% \mathrm{FCS}\end{array}$ \\
\hline $\mathrm{TiO}_{2}$ anatase & $497 \pm 290.2(0.5)$ & $170 \pm 65(0.5)$ & $-29.6 \pm 1.35$ & $-11.8 \pm 0.1$ \\
$\mathrm{TiO}_{2}$ rutile & $599 \pm 512(0.3)$ & $176 \pm 5.8(0.5)$ & $-36.7 \pm 3.7$ & $-13.4 \pm 0.7$ \\
$\mathrm{Fe}_{2} \mathrm{O}_{3}$ & $991 \pm 286(0.3)$ & $423 \pm 139(0.5)$ & $-32.3 \pm 0.4$ & $-10.1 \pm 0.5$ \\
$\mathrm{SiO}_{2}$ & $606 \pm 184(0.3)$ & $246 \pm 67(0.5)$ & $-46.1 \pm 1.2$ & $-9.5 \pm 0.3$ \\
$\mathrm{Ag}$ & $65 \pm 2.0(0.2)$ & $74 \pm 2.2(0.3)$ & $-44.7 \pm 3.6$ & $-8.9 \pm 0.7$ \\
$\mathrm{Mn}_{2} \mathrm{O}_{3}$ & $662 \pm 280(0.4)$ & $313 \pm 107(0.3)$ & $-33.6 \pm 2.5$ & $-17.1 \pm 2.5$ \\
$\mathrm{Cr}_{2} \mathrm{O}_{3}$ & $460 \pm 101(0.3)$ & $153 \pm 19(0.5)$ & $-35.6 \pm 3.9$ & $-14.3 \pm 0.5$ \\
$\mathrm{CuO}_{\mathrm{Co}} \mathrm{O}_{4}$ & $491 \pm 334(0.3)$ & $205 \pm 124(0.5)$ & $-36.3 \pm 2.5$ & $-15.8 \pm 0.4$ \\
$\mathrm{NiO}_{\mathrm{None}}$ & $1285 \pm 827(0.5)$ & $224 \pm 113(0.6)$ & $-37.6 \pm 0.6$ & $-13.1 \pm 0.9$ \\
\hline
\end{tabular}

\section{Effects of metal (oxide) food additive NPs on RAW264.7 cells}

NP forms of food additives $\mathrm{Fe}_{2} \mathrm{O}_{3}, \mathrm{SiO}_{2}, \mathrm{Ag}$ and $\mathrm{TiO}_{2}$ anatase and $\mathrm{TiO}_{2}$ rutile NPs were tested for their effects on cell viability, ROS production, mitochondrial membrane integrity (as measured by MPTP opening), intracellular ATP levels and TNF- $\alpha$ production in RAW264.7 cells upon 24 hours of exposure. Data obtained for the positive controls of the tests are presented in the supplementary figure 1. When dispersing the NPs in the medium, sedimentation was observed using light microscopy at the concentrations of $50 \mu \mathrm{g} / \mathrm{ml}$ and higher (Supplementa- 
ry figure 2). The results reveal that of the different $\mathrm{NP}$ forms of food additives only $\mathrm{SiO}_{2}$ and Ag NPs cause a significant concentration-dependent decrease in cell viability (Figure 2A). No effect on cell viability was noticed when RAW264.7 cells were exposed to the other NPs to concentrations up to $200 \mu \mathrm{g} / \mathrm{ml}$ (Figure 2A). Besides affecting cell viability, Ag NPs and $\mathrm{SiO}_{2}$ NPs also significantly decreased the intracellular ATP levels in a concentration-dependent manner (Figure 2D). $\mathrm{SiO}_{2}$ NPs caused at the highest concentration tested an increase in TNF- $\alpha$ production by the cells (Figure 2E). None of the food-related NPs showed statistically significant effects on mitochondrial membrane integrity (Figure 2C). Only $\mathrm{Fe}_{2} \mathrm{O}_{3}$ NPs significantly induced ROS production at the highest concentration tested (Figure 2B).

\section{Effects of other (non-food-related) metal oxide NPs on RAW264.7 cells}

To assess possible adverse effects of other metal oxide NPs, the effects of an additional series of metal oxide NPs were characterized on RAW264.7 cells, being $\mathrm{Mn}_{2} \mathrm{O}_{3}, \mathrm{CuO}, \mathrm{Cr}_{2} \mathrm{O}_{3}, \mathrm{Co}_{3} \mathrm{O}_{4}$ and $\mathrm{NiO}$ NPs Results obtained reveal that only exposure to $\mathrm{Mn}_{2} \mathrm{O}_{3}$ and $\mathrm{CuO}$ NPs induced significant effects in RAW264.7 cells (Figure 3). No effects were found upon exposure to $\mathrm{Cr}_{2} \mathrm{O}_{3}$, $\mathrm{Co}_{3} \mathrm{O}_{4}$ and $\mathrm{NiO}$ NPs up to concentrations of $200 \mu \mathrm{g} / \mathrm{ml}$. Exposure to $\mathrm{Mn}_{2} \mathrm{O}_{3}$ and $\mathrm{CuO} \mathrm{NPs}$ resulted in a statistically significant decrease in cell viability (starting from 200 and $50 \mu \mathrm{g} / \mathrm{ml}$ respectively; Figure 3A), a decrease of mitochondrial integrity (starting from 100 and $50 \mu \mathrm{g} /$ $\mathrm{ml}$ respectively; Figure $3 \mathrm{C}$ ) and a decrease in intracellular ATP levels (starting from 50 and $100 \mu \mathrm{g} / \mathrm{ml}$ respectively; Figure 3D). $\mathrm{Mn}_{2} \mathrm{O}_{3}$ and $\mathrm{CuO}$ NPs also caused a significant increase in ROS production (Figure 3B). Further, a concentration-dependent increase of TNF- $\alpha$ production upon $\mathrm{Mn}_{2} \mathrm{O}_{3}$ NPs exposure was found, but these effects were not statistically significant (Figure 3E). 

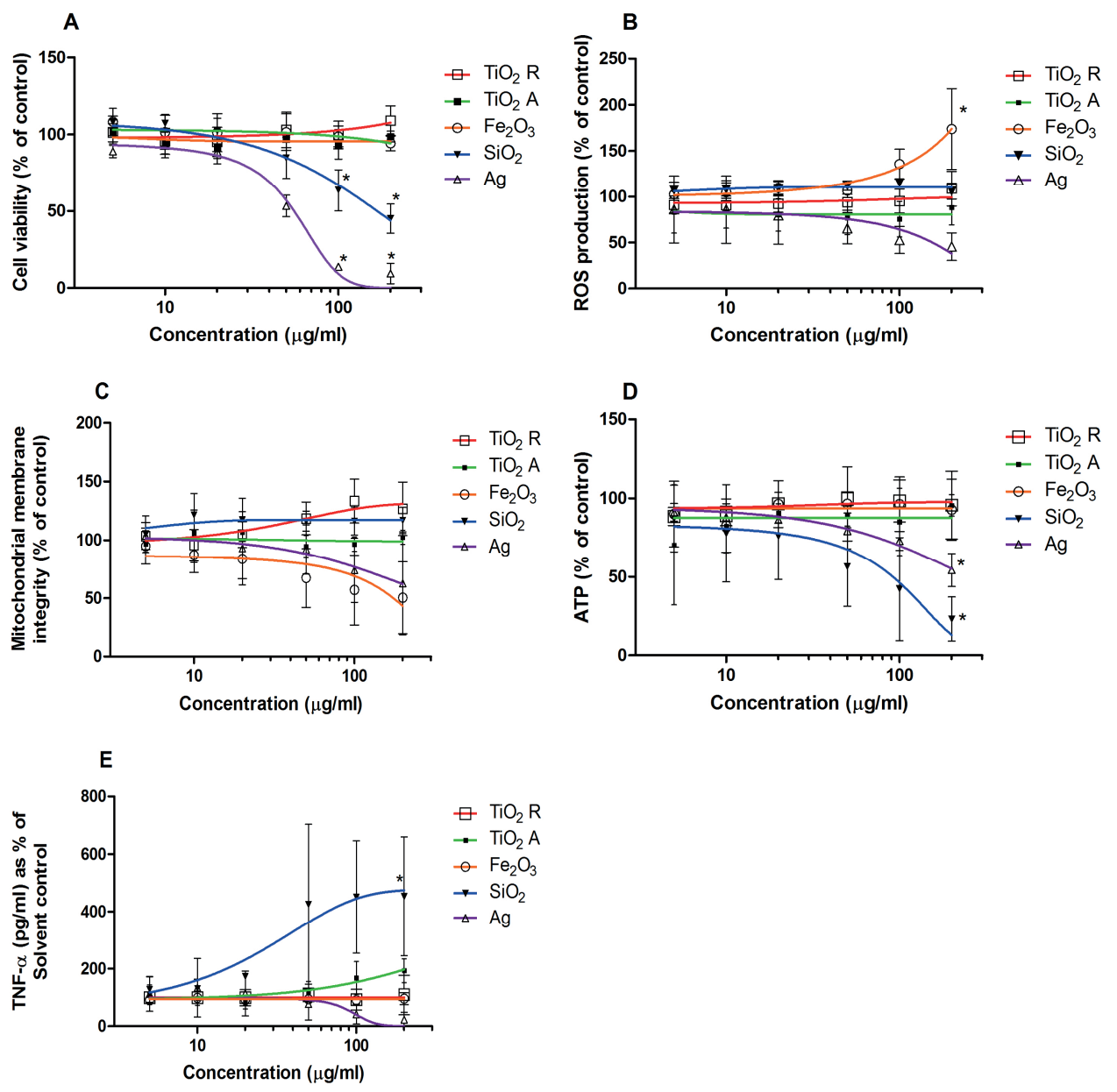

Figure 2. Effects of different NP forms of metal (oxide) food additives on: A) cell viability, B) ROS producti on, C) mitochondrial membrane integrity, D) ATP levels and E) TNF- $\alpha$ production in RAW264.7

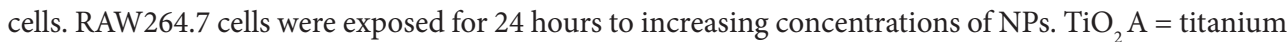
dioxide anatase $\mathrm{NP} ; \mathrm{TiO}_{2} \mathrm{R}=$ titanium dioxide rutile $\mathrm{NP} ; \mathrm{Fe}_{2} \mathrm{O}_{3}=$ iron oxide $\mathrm{NP} ; \mathrm{SiO}_{2}=$ silicon dioxide $\mathrm{NP} ; \mathrm{Ag}=$ silver NP. 

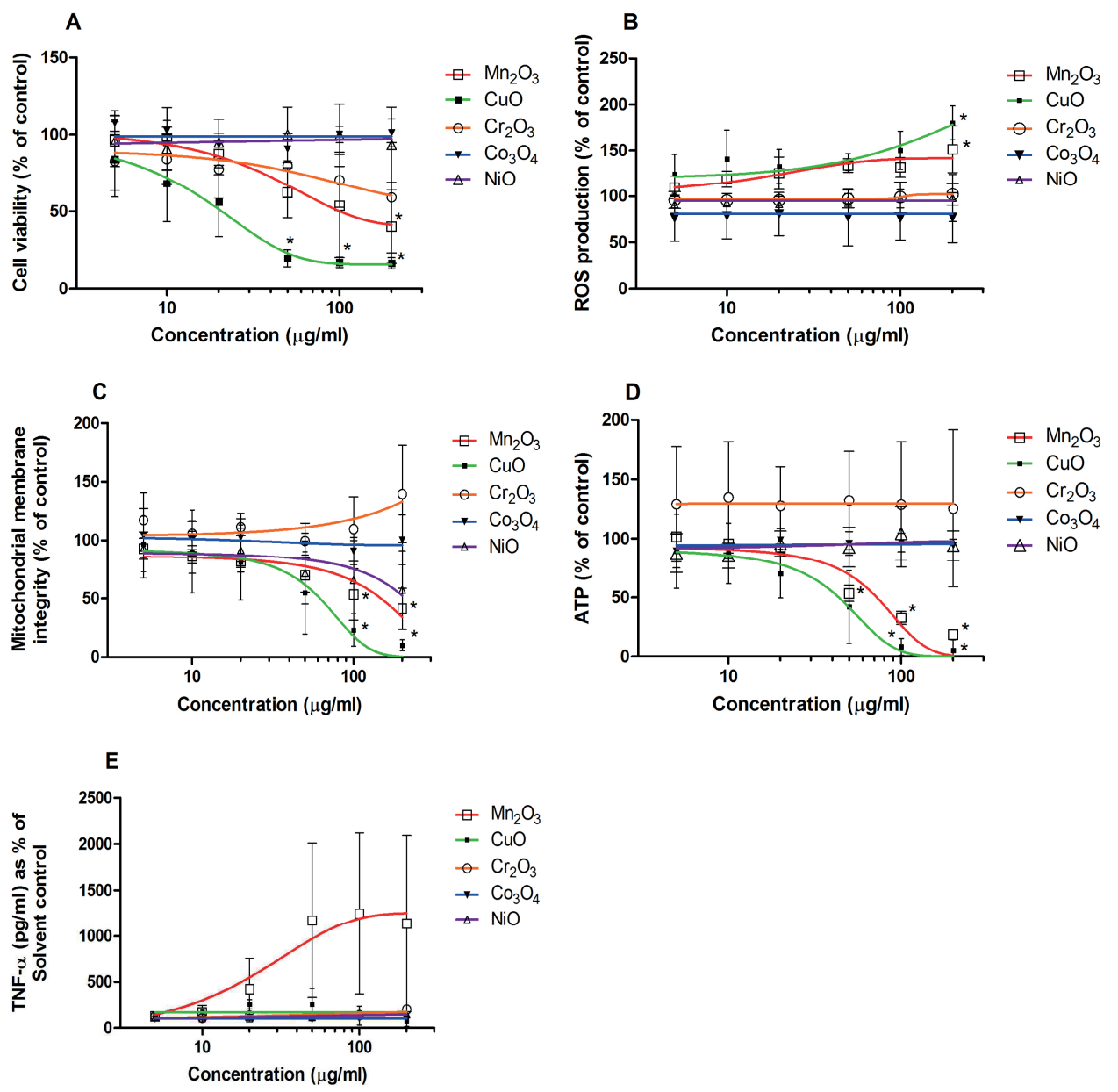

Figure 3. Effects of other (non-food-related) metal oxide NPs on A) cell viability, B) ROS production, C) mitochondrial membrane integrity, D) ATP, and E) TNF- $\alpha$ production in RAW264.7 cells. RAW264.7 cells were exposed for 24 hours to increasing concentrations of NPs. $\mathrm{Mn}_{2} \mathrm{O}_{3}=$ manganese oxide NP; $\mathrm{CuO}=$ copper oxide $\mathrm{NP} ; \mathrm{Cr}_{2} \mathrm{O}_{3}=$ chromium oxide $\mathrm{NP} ; \mathrm{Co}_{3} \mathrm{O}_{4}=$ cobalt oxide $\mathrm{NP} ; \mathrm{NiO}=$ nickel oxide $\mathrm{NP}$. 


\section{DISCUSSION}

The present study assessed the adverse effects of metal (oxide) NPs on macrophages in vitro, to study possible hazards of such NPs. To this end the effects of a series of food-related and other (non-food-related) metal (oxide) NPs on cell viability, ROS production, mitochondrial integrity, intracellular ATP levels and TNF- $\alpha$ production in RAW264.7 macrophages were assessed. The results indicate that of the food additive related $\mathrm{NPs}, \mathrm{SiO}_{2}$ and $\mathrm{Ag} \mathrm{NPs}$ were the most toxic, showing a significant decrease of cell viability at concentrations of $100 \mu \mathrm{g} / \mathrm{ml}$ and higher, and a decrease of intracellular ATP at $200 \mu \mathrm{g} / \mathrm{ml}$ (Figure 2). In addition, $\mathrm{SiO}_{2} \mathrm{NPs}$ induced increased TNF- $\alpha$ production at $200 \mu \mathrm{g} / \mathrm{ml}$. From the series of other (non-food-related) metal oxide NPs, $\mathrm{CuO}$ and $\mathrm{Mn}_{2} \mathrm{O}_{3}$ NPs were the most toxic, affecting mitochondrial integrity, ROS production, ATP levels and cell viability at concentrations of $50 \mu \mathrm{g} / \mathrm{ml}$ and higher. Altogether, the present study shows no or limited effects of the NP forms of metal (oxide) food additives and related NPs on cell viability, ROS production, mitochondrial integrity, intracellular ATP levels and TNF- $\alpha$ production in RAW264.7 macrophages.

In the present study, effects for most NPs were only detected at high concentrations, if detected at all. At concentrations of $50 \mu \mathrm{g} / \mathrm{ml}$ and higher, metal oxide NP sedimentation was observed (supplementary figure 2), and it has been reported that toxicity of NPs may be affected by their agglomeration (Oberdorster 2010, Albanese et al. 2011, Zook et al. 2011, Caballero-Díaz et al. 2013). However, since these NP agglomerations appeared to be co-localized with the RAW264.7 marcrophages and since they were not dispersed in the medium, this may indicate that these agglomerations are not the result of dispersity problems, but that the macrophages may have internalised the NPs. Therefore, the toxicity of NPs to the macrophages as observed in the present study for $\mathrm{SiO}_{2}, \mathrm{Ag}, \mathrm{CuO}$ and $\mathrm{Mn}_{2} \mathrm{O}_{3} \mathrm{NPs}$ may be related to a high NP uptake, as has been reported before for gold NPs (Zhang et al. 2011).

It has been hypothesized that oxidative stress is an important mechanism underlying the cytotoxicity of NPs, including $\mathrm{Mn}_{2} \mathrm{O}_{3}$ and CuO NPs (Carlson et al. 2008, Park et al. 2009, Foucaud et al. 2010). NPs' ability to generate oxidative stress can be due to their high surface reactivity (Krug et al. 2011), the presence of transition metal ions in the case of metal oxides and metal NPs (Kim et al. 2014), the fact that they can interact with the mitochondrial membrane and disrupt the electron transport chain (Elsaesser et al. 2012), and because they can attract macrophages and neutrophils that in response produce ROS (Krug et al. 2011). For $\mathrm{Mn}_{2} \mathrm{O}_{3}$ NPs and $\mathrm{CuO}$ NPs, we indeed found an increase in ROS production upon exposure of the cells (Figure 3B), which was accompanied by a decrease in cell viability at high exposure concentrations. The increase in ROS production upon $\mathrm{Mn}_{2} \mathrm{O}_{3} \mathrm{NPs}$ and $\mathrm{CuO}$ NPs exposure was clearly associated with a decrease in mitochondrial membrane integrity (Figure 3C) and a decrease in ATP levels (Figure 3D) suggesting that the ROS production may originate 
from impairment of the membrane integrity resulting in uncoupling of the electron transport chain. For the other two most cytotoxic NPs tested in the present study, the food-related $\mathrm{SiO}_{2}$ NPs and Ag NPs, no increased ROS production was found (Figure 2B). Also, no effects on mitochondrial membrane integrity were observed for these NPs (Figure 2C) and only slight effects on ATP levels (Figure 2D), which may be a secondary effect because of dying cells. This indicates that the food-related $\mathrm{Ag}$ NPs and $\mathrm{SiO}_{2}$ NPs likely have a different mode of action underlying their cytotoxicity than the non-food related $\mathrm{Mn}_{2} \mathrm{O}_{3}$ NPs and $\mathrm{CuO}$ NPs.

Of the NPs used in the present study, Ag NPs and $\mathrm{CuO}$ NPs were the most toxic ones. $\mathrm{CuO}$ and Ag NPs have been reported before as being significantly cytotoxic to A549 cells and Calu3 epithelial cells and Raw264.7 macrophages (Karlsson et al. 2008, Haase et al. 2011, Rotoli et al. 2012). It is known that ions are released from $\mathrm{Ag}$ and $\mathrm{CuO}$ NPs at different rates, making the understanding of the delivered dose challenging (Ponti et al. 2009, Misra et al. 2012). Discussion remains about the role of this ion dissolution in the mode of action underlying the toxicity (Karlsson et al. 2008, Adamczyk et al. 2016). Although toxicity of CuO NPs may be (partly) caused by dissolution of $\mathrm{Cu}$ ions, a study of Karlsson et al. (2008) showed that the toxicity of $\mathrm{CuO}$ NPs cannot be solely explained by $\mathrm{Cu}$ ions released into the cell medium. For example, cell viability of A549 cells decreased more when exposed to $40 \mu \mathrm{g} / \mathrm{mL}$ of $\mathrm{Cu}$ from $\mathrm{CuO} N$ Ps $(50 \mu \mathrm{g} / \mathrm{mL} \mathrm{CuO})$ than when exposed to $40 \mu \mathrm{g} / \mathrm{mL}$ of $\mathrm{Cu}$ from $\mathrm{CuCl}_{2}(85 \mu \mathrm{g} / \mathrm{mL}$ $\mathrm{CuCl}_{2}$ ) (Karlsson et al. 2008). Regarding Ag NPs, no toxic effect was observed when HeLa cells were exposed to Ag ions (as silver nitrate) up to $8 \mu \mathrm{g} / \mathrm{mL}$ silver nitrate whereas Ag NPs caused toxicity starting from $2.0 \mu \mathrm{g} / \mathrm{mL}$ (Kaba and Egorova 2015). For U937 cells on the other hand, Ag ions showed similar toxicity as Ag NPs in a concentration range of $0.5-8 \mu \mathrm{g} / \mathrm{mL}$ (Kaba et al. 2015). So although it is known that ions can be released from these NPs, it remains still a topic of investigation how these ions contribute to the toxicity observed.

Our results showed that $\mathrm{SiO}_{2} \mathrm{NPs}$ were the most toxic ones among the tested food-related NPs. $\mathrm{SiO}_{2} \mathrm{NPs}$ are known to exert a decrease in cell viability in primary human monocyte-derived macrophages and MH-S and RAW264.7 cells (Farcal et al. 2015, Di Cristo et al. 2016). Exposure to food-related NPs is expected to be mainly via the gastrointestinal tract. Upon oral exposure, NP characteristics may change because of gastrointestinal digestion (Rogers et al. 2012, Walczak et al. 2015). When food products containing $\mathrm{SiO}_{2}$ were tested in an in vitro digestion model, simulating the conditions of the human gastrointestinal tract, it was found that $\mathrm{SiO}_{2}$ NPs (5-200 nm) were still present in the intestinal content (Peters et al. 2012). This indicates that it is most likely that upon consumption of foods containing particulate $\mathrm{SiO}_{2}$, the gut epithelium is exposed to $\mathrm{SiO}_{2} \mathrm{NPs}$ (5-200 nm) (Peters et al. 2012). To enter the systemic circulation, NPs need to be absorbed in the gastrointestinal tract. Intestinal absorption largely depends on the size of NPs, surface charge and hydrophobicity (Bouwmeester et al. 2011, Bhattacharjee et al. 2013). In an in vivo study in rats it was shown that 6.6-9.7\% of $\mathrm{SiO}_{2} \mathrm{NPs}$ 
of 20 and $100 \mathrm{~nm}$ were absorbed into the systemic circulation when orally administered at a single dose of 500 and $1000 \mathrm{mg} / \mathrm{kg}$ (Lee et al. 2014), indicating that $\mathrm{SiO}_{2} \mathrm{NPs}$ can get translocated across the intestinal barrier. An in vitro study showed that $50 \mathrm{~nm} \mathrm{SiO}{ }_{2} \mathrm{NPs}_{\text {sere }}$ able to translocate across an intestinal barrier based on results obtained in a Caco-2 cell transwell model system (Sakai-Kato et al. 2014).

With an estimated daily intake of $\mathrm{SiO}_{2}$ in the nano-size range of $1.8 \mathrm{mg} / \mathrm{kg}$ bw/day (van der Zande et al. 2014, van Kesteren et al. 2014), the daily intake of a $70 \mathrm{~kg}$ person would be 126 $\mathrm{mg}$. In the adult human the gastrointestinal tract represents a considerable surface area of about $200 \mathrm{~m}^{2}$ which has the potential to interact with ingested NPs (Bergin et al. 2013). In addition the gastrointestinal tract has localised representations of the adaptive immune system in the form of gut-associated lymphoid tissue (GALT) aggregates (Peyer's Patches) (Bergin and Witzmann 2013). These Peyer's patches account for $1 \%$ of the total surface area of the gastrointestinal tract (des Rieux et al. 2006). About 9 litres of fluid pass through the GI system each day (Sherwood L. 2014). Thus, upon oral intake of $126 \mathrm{mg} \mathrm{SiO}_{2}$ per day this would lead to a concentration of $14 \mu \mathrm{g} / \mathrm{ml}$, which is lower than the lowest observed adverse effect concentration of $100 \mu \mathrm{g} / \mathrm{mL}$ in the present study.

If $100 \%$ of the estimated daily intake of $\mathrm{SiO}_{2} \mathrm{NPs}(126 \mathrm{mg})$ would be transported from the intestines into the blood, assuming 5 liters of blood and a $100 \%$ clearance per day, a maximal concentration of $25 \mu \mathrm{g} / \mathrm{ml}$ would be reached if NPs would not distribute to tissues. Also this concentration is lower than the lowest observed adverse effect concentration of $100 \mu \mathrm{g} / \mathrm{mL}$ defined in the present study. Considering that the oral bioavailability is lower than $10 \%$ and that NPs also distribute to tissues (Lee et al. 2014), concentrations higher than $2.5 \mu \mathrm{g} / \mathrm{mL}$ are not likely to be reached in a real-life exposure scenario. This concentration is far below the concentrations of $\mathrm{SiO}_{2}$ causing in vitro toxicity in the present study, suggesting that in vivo toxic effects of food additive related $\mathrm{SiO}_{2} \mathrm{NPs}$ on macrophages are expected to be limited. This is all in line with the results from an oral repeated-dose toxicity study in which male Wistar rats were exposed to $\mathrm{SiO}_{2} \mathrm{NPs}$ via the diet for 28 or 84 days, showing no toxicity at the highest dose level tested (2500 mg/kg bw/day) (van der Zande et al. 2014).

It has to be kept in mind that NPs may not only affect the gastrointestinal cells directly but may also influence the microbiome, although the evaluation of effects of NPs on the microbiome is in its early infancy (Pietroiusti et al. 2015). For example, the cytotoxicity of Ag NPs on Escherichia coli as representative for intestinal bacteria was compared with the cytotoxicity of Ag NPs on gastrointestinal cells. This revealed that Ag NPs affected bacteria at lower concentrations than those that affected gastrointestinal cells, indicating that Ag NPs may cause adverse effects by selectively affecting the gut microbiota (Frohlich et al. 2016). In a study using an in vitro colon model (Taylor et al. 2015), $\mathrm{TiO}_{2}, \mathrm{ZnO}$ and $\mathrm{CeO}_{2} \mathrm{NPs}$ induced significant 
phenotypic changes of the gut microbial community, inlcuding the production of short chain fatty acids which are the key metabolites in the control of energy homeostatis and which have been implicated in the development of obesity (Schwiertz et al. 2010). For other food related metal (oxide) NPs such as $\mathrm{SiO}_{2}, \mathrm{Fe}_{2} \mathrm{O}_{3}$ and $\mathrm{Ag} \mathrm{NPs}$, such effects on the gut microbiota and related short chain fatty acid production remain to be investigated.

\section{CONCLUSION}

In conclusion, the present study shows no or limited effects of the NP forms of metal (oxide) food additives on cell viability, ROS production, mitochondrial integrity, ATP levels and TNF- $\alpha$ production in RAW264.7 macrophages. Of the food additive related $\mathrm{NPs}, \mathrm{SiO}_{2}$ and $\mathrm{Ag}$ NPs were the most toxic, showing a decrease of cell viability at concentrations of $100 \mu \mathrm{g} / \mathrm{ml}$ and higher. Effects were only observed at high concentrations that may not be physiologically relevant, indicating that related adverse effects upon exposure to the respective NPs may be limited.

\section{ACKNOWLEDGEMENTS}

This work is supported by NanoNextNL, a micro and nanotechnology consortium of the Government of the Netherlands and 130 partners. 


\section{REFERENCES}

(2011). "Regulation EU no 1169/2011 on the provision of food information to consumers. Official Journal of the European Union. ." Official Journal of the European Union from http://eur-lex.europa.eu/legal-content/EN/ TXT/PDF/?uri=CELEX:32011R1169\&from=EN

Adamczyk, Z., M. Ocwieja, H. Mrowiec, S. Walas and D. Lupa (2016). “Oxidative dissolution of silver nanoparticles: A new theoretical approach.” J Colloid Interface Sci 469: 355-364.

Albanese, A. and W. C. Chan (2011). "Effect of gold nanoparticle aggregation on cell uptake and toxicity." ACS Nano 5(7): 5478-5489.

Athinarayanan, J., V. S. Periasamy, M. A. Alsaif, A. A. Al-Warthan and A. A. Alshatwi (2014). "Presence of nanosilica (E551) in commercial food products: TNF-mediated oxidative stress and altered cell cycle progression in human lung fibroblast cells." Cell biology and toxicology 30(2): 89-100.

Bergin, I. L. and F. A. Witzmann (2013). "Nanoparticle toxicity by the gastrointestinal route: evidence and knowledge gaps.” International journal of biomedical nanoscience and nanotechnology 3(1-2).

Bhattacharjee, S., D. Ershov, J. Gucht, G. M. Alink, I. M. Rietjens, H. Zuilhof and A. T. Marcelis (2013). "Surface charge-specific cytotoxicity and cellular uptake of tri-block copolymer nanoparticles." Nanotoxicology 7(1): 71-84.

Bhattacharjee, S., I. M. Rietjens, M. P. Singh, T. M. Atkins, T. K. Purkait, Z. Xu, S. Regli, A. Shukaliak, R. J. Clark, B. S. Mitchell, G. M. Alink, A. T. Marcelis, M. J. Fink, J. G. Veinot, S. M. Kauzlarich and H. Zuilhof (2013). "Cytotoxicity of surface-functionalized silicon and germanium nanoparticles: the dominant role of surface charges." Nanoscale 5(11): 4870-4883.

Bonora, M. and P. Pinton (2014). "The mitochondrial permeability transition pore and cancer: molecular mechanisms involved in cell death." Frontiers in oncology 4: 302.

Bouwmeester, H., S. Dekkers, M. Y. Noordam, W. I. Hagens, A. S. Bulder, C. de Heer, S. E. ten Voorde, S. W. Wijnhoven, H. J. Marvin and A. J. Sips (2009). "Review of health safety aspects of nanotechnologies in food production.” Regul Toxicol Pharmacol 53(1): 52-62.

Bouwmeester, H., J. Poortman, R. J. Peters, E. Wijma, E. Kramer, S. Makama, K. Puspitaninganindita, H. J. Marvin, A. A. Peijnenburg and P. J. Hendriksen (2011). "Characterization of translocation of silver nanoparticles and effects on whole-genome gene expression using an in vitro intestinal epithelium coculture model." ACS Nano 5(5): 4091-4103.

Caballero-Díaz, E., C. Pfeiffer, L. Kastl, P. Rivera-Gil, B. Simonet, M. Valcárcel, J. Jiménez-Lamana, F. Laborda and W. J. Parak (2013). "The Toxicity of Silver Nanoparticles Depends on Their Uptake by Cells and Thus on Their Surface Chemistry.” Particle \& Particle Systems Characterization 30(12): 1079-1085.

Carlson, C., S. M. Hussain, A. M. Schrand, L. K. Braydich-Stolle, K. L. Hess, R. L. Jones and J. J. Schlager (2008). "Unique cellular interaction of silver nanoparticles: size-dependent generation of reactive oxygen species." J Phys Chem B 112(43): 13608-13619.

Dang, T., T. Le, T. Hoang and T. Mai (2015). "Synthesis of nanostructured manganese oxides based materials and application for supercapacitor." Advances in Natural Sciences; Nanoscience and Nanotechnology 6.

des Rieux, A., V. Fievez, M. Garinot, Y. J. Schneider and V. Preat (2006). "Nanoparticles as potential oral delivery systems of proteins and vaccines: a mechanistic approach." J Control Release 116(1): 1-27.

Di Cristo, L., D. Movia, M. G. Bianchi, M. Allegri, B. M. Mohamed, A. P. Bell, C. Moore, S. Pinelli, K. Rasmussen, J. Riego-Sintes, A. Prina-Mello, O. Bussolati and E. Bergamaschi (2016). "Proinflammatory Effects of Pyrogenic and Precipitated Amorphous Silica Nanoparticles in Innate Immunity Cells.” Toxicol Sci 150(1): 40-53.

Elsabahy, M. and K. L. Wooley (2013). "Cytokines as biomarkers of nanoparticle immunotoxicity." Chemical Society reviews 42(12): 5552-5576.

Elsaesser, A. and C. V. Howard (2012). “Toxicology of nanoparticles.” Adv Drug Deliv Rev 64(2): 129-137.

Farcal, L., F. Torres Andon, L. Di Cristo, B. M. Rotoli, O. Bussolati, E. Bergamaschi, A. Mech, N. B. Hartmann, K. Rasmussen, J. Riego-Sintes, J. Ponti, A. Kinsner-Ovaskainen, F. Rossi, A. Oomen, P. Bos, R. Chen, R. Bai, C. Chen, L. Rocks, N. Fulton, B. Ross, G. Hutchison, L. Tran, S. Mues, R. Ossig, J. Schnekenburger, L. Campagnolo, L. Vecchione, A. Pietroiusti and B. Fadeel (2015). "Comprehensive In Vitro Toxicity Testing of a Panel of Representative Oxide Nanomaterials: First Steps towards an Intelligent Testing Strategy." PLoS One 10(5): e0127174. 
Faust, J. J., K. Doudrick, Y. Yang, P. Westerhoff and D. G. Capco (2014). "Food grade titanium dioxide disrupts intestinal brush border microvilli in vitro independent of sedimentation.” Cell biology and toxicology 30(3): 169-188.

Foucaud, L., S. Goulaouic, A. Bennasroune, P. Laval-Gilly, D. Brown, V. Stone and J. Falla (2010). “Oxidative stress induction by nanoparticles in THP-1 cells with 4-HNE production: stress biomarker or oxidative stress signalling molecule?” Toxicol In Vitro 24(6): 1512-1520.

Frohlich, E. E. and E. Frohlich (2016). "Cytotoxicity of Nanoparticles Contained in Food on Intestinal Cells and the Gut Microbiota." International journal of molecular sciences 17(4).

Haase, A., J. Tentschert, H. Jungnickel, P. Graf, A. Mantion, F. Draude, J. Plendl, M. E. Goetz, S. Galla, A. Masic, A. F. Thuenemann, A. Taubert, H. F. Arlinghaus and L. A (2011). “Toxicity of silver nanoparticles in human macrophages: uptake, intracellular distribution and cellular responses.” Journal of Physics: Conference series 304.

Kaba, S. I. and E. M. Egorova (2015). "In vitro studies of the toxic effects of silver nanoparticles on HeLa and U937 cells." Nanotechnology, science and applications 8: 19-29.

Karlsson, H. L., P. Cronholm, J. Gustafsson and L. Moller (2008). "Copper oxide nanoparticles are highly toxic: a comparison between metal oxide nanoparticles and carbon nanotubes." Chemical research in toxicology 21(9): 1726-1732.

Kim, C. S., H. D. Nguyen, R. M. Ignacio, J. H. Kim, H. C. Cho, E. H. Maeng, Y. R. Kim, M. K. Kim, B. K. Park and S. K. Kim (2014). "Immunotoxicity of zinc oxide nanoparticles with different size and electrostatic charge." Int J Nanomedicine 9 Suppl 2: 195-205.

Krug, H. F. and P. Wick (2011). "Nanotoxicology: An Interdisciplinary Challenge.” Angew Chem Int Ed Engl.

Lee, J. A., M. K. Kim, H. J. Paek, Y. R. Kim, M. K. Kim, J. K. Lee, J. Jeong and S. J. Choi (2014). "Tissue distribution and excretion kinetics of orally administered silica nanoparticles in rats.” Int J Nanomedicine 9 Suppl 2: 251-260.

Ly, J. D., D. R. Grubb and A. Lawen (2003). “The mitochondrial membrane potential (deltapsi(m)) in apoptosis; an update." Apoptosis 8(2): 115-128.

Ma, J., T. Chen, J. Mandelin, A. Ceponis, N. E. Miller, M. Hukkanen, G. F. Ma and Y. T. Konttinen (2003). "Regulation of macrophage activation." Cellular and molecular life sciences : CMLS 60(11): 2334-2346.

Misra, S. K., A. Dybowska, D. Berhanu, S. N. Luoma and E. Valsami-Jones (2012). "The complexity of nanoparticle dissolution and its importance in nanotoxicological studies." The Science of the total environment 438 : 225-232.

Oberdorster, G. (2010). “Safety assessment for nanotechnology and nanomedicine: concepts of nanotoxicology." J Intern Med 267(1): 89-105.

Park, E. J. and K. Park (2009). "Oxidative stress and pro-inflammatory responses induced by silica nanoparticles in vivo and in vitro." Toxicol Lett 184(1): 18-25.

Park, E. J., J. Yi, K. H. Chung, D. Y. Ryu, J. Choi and K. Park (2008). "Oxidative stress and apoptosis induced by titanium dioxide nanoparticles in cultured BEAS-2B cells." Toxicol Lett 180(3): 222-229.

Periasamy, V. S., J. Athinarayanan, A. M. Al-Hadi, F. A. Juhaimi, M. H. Mahmoud and A. A. Alshatwi (2015). "Identification of titanium dioxide nanoparticles in food products: induce intracellular oxidative stress mediated by TNF and CYP1A genes in human lung fibroblast cells." Environ Toxicol Pharmacol 39(1): 176-186.

Peters, R., E. Kramer, A. G. Oomen, Z. E. Rivera, G. Oegema, P. C. Tromp, R. Fokkink, A. Rietveld, H. J. Marvin, S. Weigel, A. A. Peijnenburg and H. Bouwmeester (2012). "Presence of nano-sized silica during in vitro digestion of foods containing silica as a food additive." ACS Nano 6(3): 2441-2451.

Pietroiusti, A., A. Magrini and L. Campagnolo (2015). "New frontiers in nanotoxicology: Gut microbiota/microbiome-mediated effects of engineered nanomaterials." Toxicology and applied pharmacology.

Ponti, J., E. Sabbioni, B. Munaro, F. Broggi, P. Marmorato, F. Franchini, R. Colognato and F. Rossi (2009). “Genotoxicity and morphological transformation induced by cobalt nanoparticles and cobalt chloride: an in vitro study in Balb/3T3 mouse fibroblasts." Mutagenesis 24(5): 439-445.

Rogers, K. R., K. Bradham, T. Tolaymat, D. J. Thomas, T. Hartmann, L. Ma and A. Williams (2012). "Alterations in physical state of silver nanoparticles exposed to synthetic human stomach fluid." The Science of the total environment 420: 334-339. 
Rotoli, B. M., O. Bussolati, A. L. Costa, M. Blosi, L. Di Cristo, P. P. Zanello, G. Bianchi, R. Visigalli and E. Bergamaschi (2012). "Comparative effects of metal oxide nanoparticles on human airway epithelial cells and macrophages." Journal of nanoparticle research : an interdisciplinary forum for nanoscale science and technology 14(1069).

Sakai-Kato, K., M. Hidaka, K. Un, T. Kawanishi and H. Okuda (2014). "Physicochemical properties and in vitro intestinal permeability properties and intestinal cell toxicity of silica particles, performed in simulated gastrointestinal fluids." Biochim Biophys Acta 1840(3): 1171-1180.

Schwiertz, A., D. Taras, K. Schafer, S. Beijer, N. A. Bos, C. Donus and P. D. Hardt (2010). "Microbiota and SCFA in lean and overweight healthy subjects.” Obesity 18(1): 190-195.

Sharma, V., D. Anderson and A. Dhawan (2011). "Zinc oxide nanoparticles induce oxidative stress and genotoxicity in human liver cells (HepG2).” J Biomed Nanotechnol 7(1): 98-99.

Sherwood L. (2014). Human Physiology from cells to systems. Canada, Cengage Learning.

Shvedova, A. A., A. Pietroiusti, B. Fadeel and V. E. Kagan (2012). "Mechanisms of carbon nanotube-induced toxicity: focus on oxidative stress.” Toxicol Appl Pharmacol 261(2): 121-133.

Siddiqui, M. A., M. Ahamed, J. Ahmad, M. A. Majeed Khan, J. Musarrat, A. A. Al-Khedhairy and S. A. Alrokayan (2012). "Nickel oxide nanoparticles induce cytotoxicity, oxidative stress and apoptosis in cultured human cells that is abrogated by the dietary antioxidant curcumin." Food Chem Toxicol 50(3-4): 641-647.

Tavares, K., A. Caloto-Oliveira, D. Vicentini, S. Melegari and W. Matias (2014). "Acute toxicity of copper and chromium oxide nanoparticles to Daphnia similis." Ecotoxicolo. Environ. Contam. 9(1): 43-50.

Taylor, A. A., I. M. Marcus, R. L. Guysi and S. L. Walker (2015). "Metal oxide nanoparticles induce minimal phenotypic changes in a model colon gut microbiota." Environ Eng Sci 32: 602-612.

van der Zande, M., R. J. Vandebriel, M. J. Groot, E. Kramer, Z. E. Herrera Rivera, K. Rasmussen, J. S. Ossenkoppele, P. Tromp, E. R. Gremmer, R. J. Peters, P. J. Hendriksen, H. J. Marvin, R. L. Hoogenboom, A. A. Peijnenburg and H. Bouwmeester (2014). "Sub-chronic toxicity study in rats orally exposed to nanostructured silica." Part Fibre Toxicol 11: 8.

van Kesteren, P. C., F. Cubadda, H. Bouwmeester, J. C. van Eijkeren, S. Dekkers, W. H. de Jong and A. G. Oomen (2014). "Novel insights into the risk assessment of the nanomaterial synthetic amorphous silica, additive E551, in food." Nanotoxicology: 1-10.

Walczak, A. P., E. Kramer, P. J. Hendriksen, R. Helsdingen, M. van der Zande, I. M. Rietjens and H. Bouwmeester (2015). "In vitro gastrointestinal digestion increases the translocation of polystyrene nanoparticles in an in vitro intestinal co-culture model.” Nanotoxicology 9(7): 886-894.

Weir, A., P. Westerhoff, L. Fabricius, K. Hristovski and N. von Goetz (2012). “Titanium dioxide nanoparticles in food and personal care products." Environ Sci Technol 46(4): 2242-2250.

Zhang, Q., V. M. Hitchins, A. M. Schrand, S. M. Hussain and P. L. Goering (2011). "Uptake of gold nanoparticles in murine macrophage cells without cytotoxicity or production of pro-inflammatory mediators." Nanotoxicology 5(3): 284-295.

Zook, J. M., R. I. Maccuspie, L. E. Locascio, M. D. Halter and J. T. Elliott (2011). "Stable nanoparticle aggregates/ agglomerates of different sizes and the effect of their size on hemolytic cytotoxicity." Nanotoxicology 5(4): 517-530. 


\section{SUPPLEMENTARY MATERIALS CHAPTER 5}
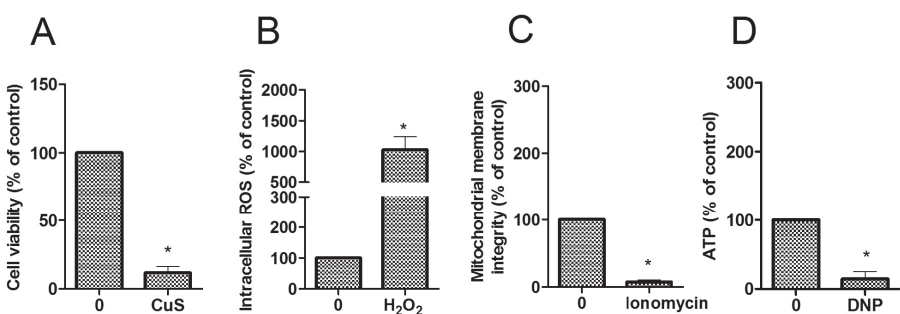

$\mathrm{E}$

Supplementary figure 1. Data obtained for the positive controls for the cell viability assay, ROS assay, MPTP assay, ATP assay and TNF- $\alpha$ assay using respectively A) $100 \mu \mathrm{M}$ copper sulfate, B) $10 \mathrm{mM}$ hydrogen peroxide, C) $10 \mu \mathrm{M}$ ionomycin, D) $4 \mathrm{mM}$ 2,4-dinitrophenol (DNP), and E) $0.1 \mu \mathrm{g} / \mathrm{ml}$ lipopolysaccharide (LPS).

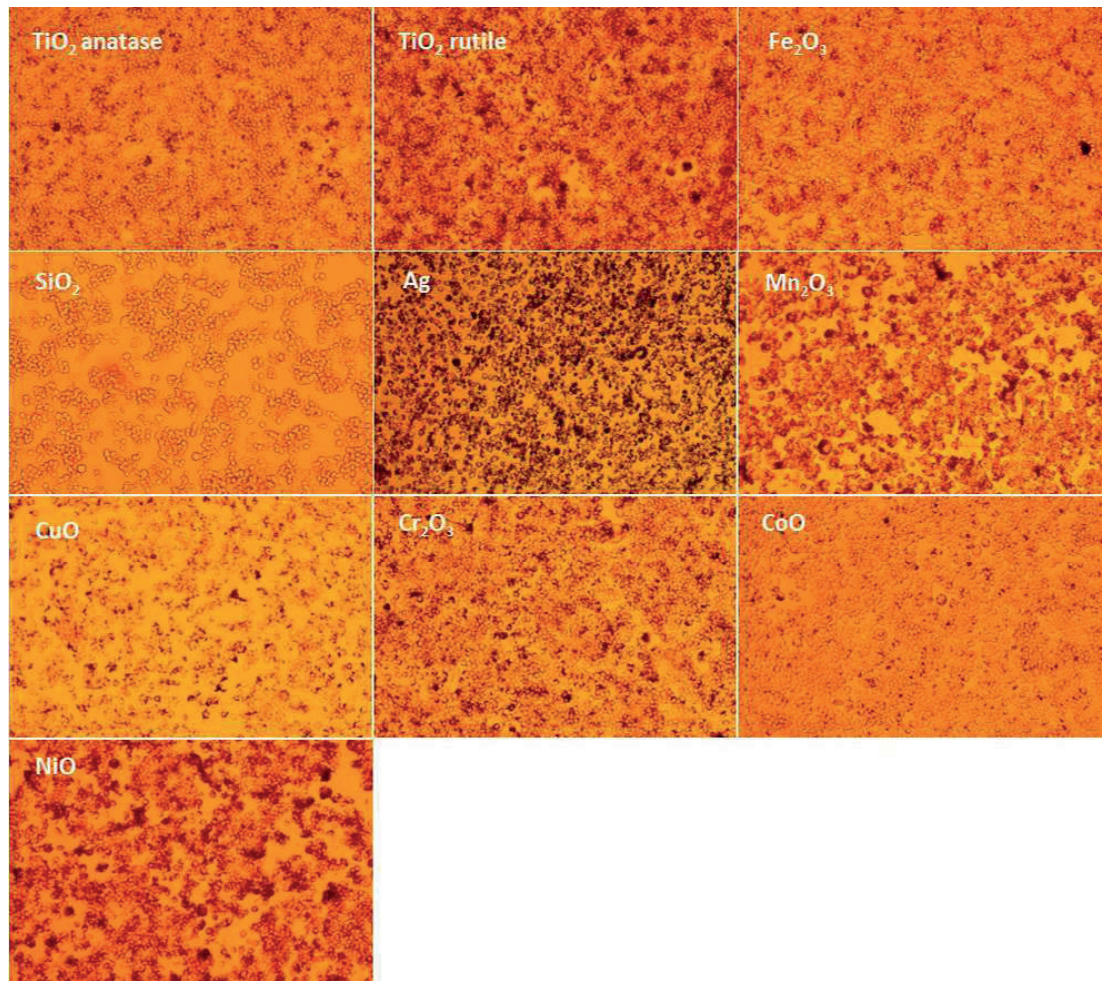

Supplementary figure 2. Microscopic pictures of RAW264.7 cells after a 24-hour exposure to metal (oxide) NPs at a concentration of $200 \mu \mathrm{g} / \mathrm{ml}$. 


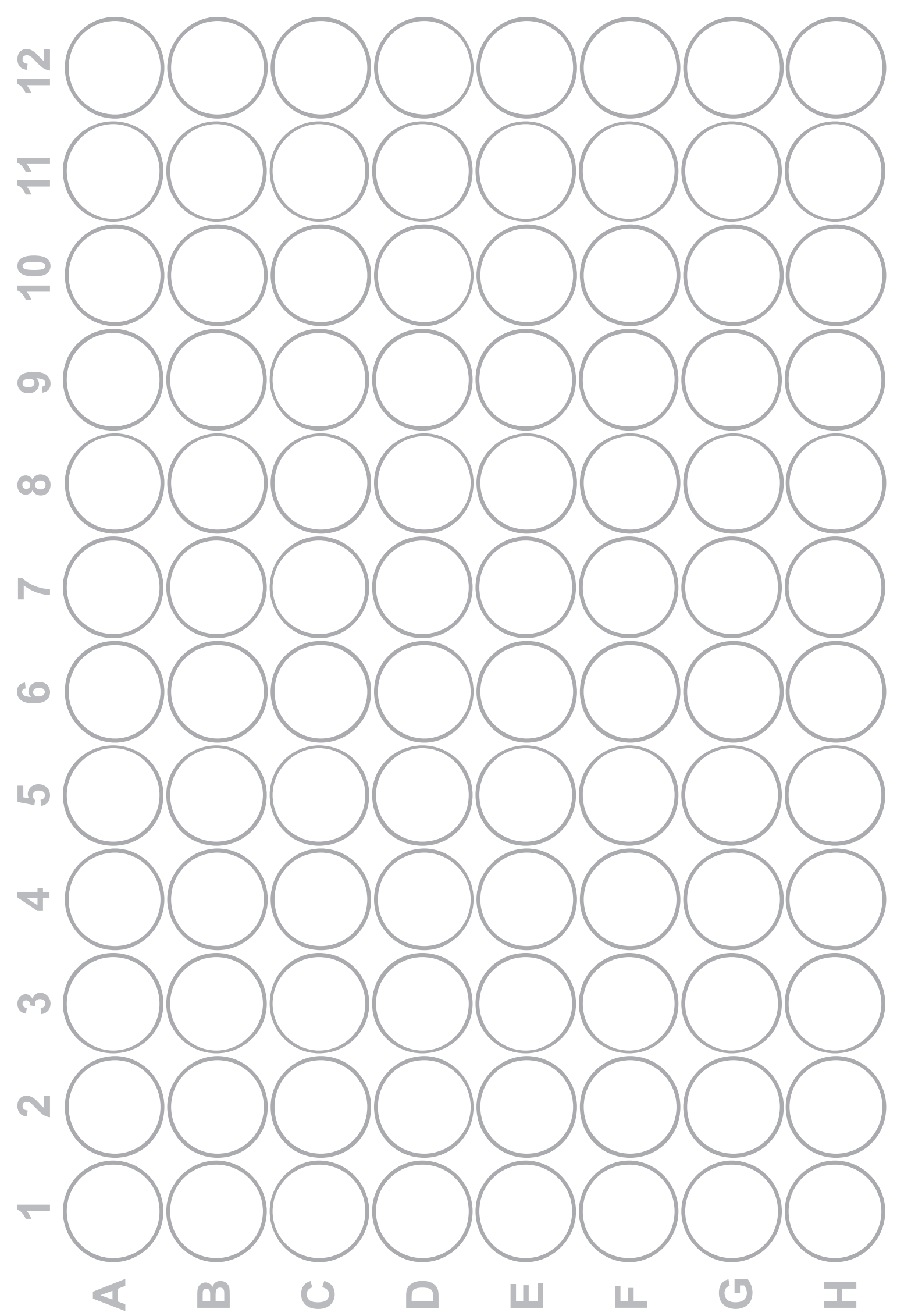




\title{
Chapter 6
}

\section{Cellular interactions of silver nanoparticles with systematic variation in size and surface coating with macrophage RAW 264.7 cells}

\author{
Sunday Makama \\ Samantha K. Kloet \\ Jordi Piella \\ Johannes H.J. van den Berg \\ Norbert C. A. de Ruijter \\ Victor F. Puntes \\ Ivonne M.C.M. Rietjens \\ Nico W. van den Brink
}




\section{ABSTRACT}

Increasing use of engineered nanoparticles has led to an increased likelihood of their environmental release and raises concern for ecosystem and health hazard. The volume of silver nanoparticle (Ag NP)-based products entering the market is relatively high, making the risk assessment of Ag NPs a priority. In literature, varying and sometimes conflicting reports exists about the physicochemical properties of NPs that are important in influencing their uptake and effects. To address this discrepancy, small and medium sized (20 and $50 \mathrm{~nm}$ ) Ag NPs with different surface coating/charges (chitosan/positive: Ag NP_Chit; bovine serum albumin/negative: Ag NP_BSA, and; polyvinylpyrrolidone/neutral: Ag NP_PVP) were specifically synthesized and characterized. Macrophage cells (RAW 264.7) were exposed to these Ag NPs at $0-200 \mu \mathrm{g} / \mathrm{mL}$, and uptake dynamics, cell viability, inductions of tumor necrosis factor (TNF)- $\alpha$ and reactive oxygen species (ROS) were assessed. Generally, adverse effects from exposure to the tested Ag NPs resulting in reduced overall viability were similar irrespective of Ag NP types or sizes. On adenosine triphosphate production and specific mechanisms of toxicity (TNF- $\alpha$ and ROS production) however, the Ag NPs differed significantly. Tests targeting specific mechanisms of action indicated the highest induction of TNF- $\alpha$ in cells exposed to both sizes of the more potent negatively charged Ag NP_BSA (80x higher than control). Significant ROS induction was only observed with the $20 \mathrm{~nm}$ positively charged $\mathrm{Ag}$ NP_Chit. Additionally, live microscopic imaging of exposed cells allowed the monitoring of the uptake dynamics and subcellular cytoplasmic accumulation of Ag NPs. The present study provides further evidence of the influence of NP properties in driving their toxicity in an in vitro model. 


\section{INTRODUCTION}

Nanotechnology can be considered as one of the fastest growing technologies which has revolutionized the industrial sector (Peterson, 2000, Gwinn and Vallyathan, 2006, Kennell, 2009), bringing about possibilities only imagined a few decades ago. This has led to increased production and applications of engineered nanoparticles (NPs) for consumer products (Wijnhoven et al., 2009, Wijnhoven et al., 2010). Presently, NPs continue to find applications in the design and manufacture of household products, electronics and medical devices, food and transport systems, to mention a few (Ahamed et al., 2010, Haider and Kang, 2015). Consequently, the increase in production and use of NPs will likely result in an increased release into the environment as industrial waste (or by-product), sewage or waste water, (Geranio et al., 2009, Wijnhoven et al., 2009, Benn et al., 2010, Reidy et al., 2013). Such environmental release could potentially have harmful impacts.

Currently, silver nanoparticles (Ag NPs) represent the nanomaterial most frequently used in products on the market (Wijnhoven et al., 2010, Vance et al., 2015) owing to their well demonstrated antimicrobial activity (Silver and Phung, 1996, Sondi and Salopek-Sondi, 2004, Lara et al., 2010, Hwang et al., 2012, Prabhu and Poulose, 2012, Franci et al., 2015). At the nanoscale $(1-100 \mathrm{~nm})$, the small size and increased surface area of Ag NPs result in novel properties. These can be enhanced by stabilization and/or functionalization using biocompatible conjugates. Essentially, the type of surface coating and process used in stabilizing engineered NPs during synthesis determines their surface charges, solubility and/or hydrophobicity (Evanoff and Chumanov, 2005, Abou El-Nour et al., 2010, Bastus et al., 2014, Shenashen et al., 2014). This in turn influences the behavior and environmental fate of NPs, as well as their effects on organisms (Kreuter, 2004, Roohani-Esfahani et al., 2010, Shoults-Wilson et al., 2011, Chanana and Liz-Marzán Luis, 2012).

Over the last two decades, researchers have attempted to elucidate the fate and effect of various NPs using both in vivo and in vitro models (Foldbjerg et al., 2012, Yu et al., 2013, van der Ploeg et al., 2014a, van der Ploeg et al., 2014b). Although the importance of the physicochemical properties of NPs in influencing their fate and toxicity has increasingly been investigated, reports in available literature have not been consistent. Important physicochemical properties in this regard include size and NPs dispersion (both mono- and polydispersity), shape, zeta potential, and agglomeration and dissolution rates (Reidy et al., 2013). While some studies have reported effects of size (Powers et al., 2011b) or charge (Suresh et al., 2012), others fail to detect these (Yang et al., 2012). Additional perspectives on the influence of physicochemical properties of Ag NPs on their interactions in both in vivo and in vitro models, has been reviewed earlier (Johnston et al., 2010, Reidy et al., 2013). From the reviews cited and examples mentioned above, one can identify the challenge associated with the regulation of Ag NPs and 
other NPs in general. This is especially so, given the limited data base and the limitations of generating in vivo data for all the different forms and types of NPs.

A better understanding of the properties that influence the fate and effects of Ag NPs in organisms therefore, will facilitate appropriate risk assessment which in turn will assist the regulation of nanomaterials. Using in vitro models in risk assessments of chemicals facilitates the setting of priorities for in vivo testing, thereby reducing the number of animals required (Braakhuis et al., 2015). By first investigating with in vitro models, insights in the toxicity and possible mode of action of NPs are obtained. Such information could be used in a weight of evidence approach (Connolly et al., 2015), furthering our understanding of observations made under in vivo situations, as well as defining priorities for in vivo testing and facilitating read-across.

In this study, we systematically investigated the physicochemical properties of Ag NPs influencing their interactions at cellular level, using an in vitro mammalian cell line model: the mouse monocyte macrophage (RAW 264.7). Macrophages are involved in the defense system of an organism against infections, and the role of Ag NP properties in initiating inflammatory (TNF- $\alpha$ ) and oxidative stress responses in these cells were investigated. Since it appears that how a NP is produced influences both its fate and effect following exposures, specifically prepared Ag NPs were used in the current study. These were synthesized to differ in size and surface chemistry (charge), two important properties influencing NP uptake and effect (Reidy et al., 2013). Using imaging techniques, we also showed the uptake dynamics of Ag NPs at a cellular level. The outcome of this study provides further insight into how Ag NP properties determine their fate and effects at cellular level, thus indirectly shedding light on what the outcome may likely be in intact organisms (Landsiedel et al., 2012, Cornelis et al., 2014).

\section{MATERIALS AND METHODS}

\section{Experimental design}

The mouse monocyte macrophage cell line (RAW 264.7) was used as the in vitro model, and cells were treated with Ag NPs. Experiments were conducted in three independent exposures for each type of $\mathrm{Ag} \mathrm{NP}(\mathrm{n}=3)$ and the data generated were processed with Microsoft Excel. Where appropriate, the data were subjected to one-way analysis of variance (ANOVA) with the aid of GraphPad Prism 5.04 for Windows (GraphPad Software, San Diego California US, www.graphpad.com), and logistic regression was done using GenStat 17th ed. (17.1.0.14713; VSN International, Hemel Hempstead, UK, GenStat.co.uk). A $p$ value of $<0.05$ was considered significant. 


\section{Reagents and Instruments}

All chemicals, enzymes and reagents were analytical grade and purchased from Sigma-Aldrich $^{\circledast}$ (Zwijndrecht, NL), unless where stated otherwise. Apart from one commercially obtained Ag NP (Ag NP_NC), all other Ag NPs were synthesized at the Catalan Institute of Nanoscience and Nanotechnology (ICN2, Barcelona, ES) by methods described below. Endpoint measurements were carried out using SpectraMax M2 Microplate Reader (Molecular Devices LLC, Sunnyvale, CA US) for fluorescence and absorbance, or Luminoskan Ascent (Thermo Scientific, 5300172, MA, US) for luminescence.

\section{Synthesis of Ag NPs}

Colloidal, dispersed Ag NPs of two different sizes (20 and $50 \mathrm{~nm}$ ) were prepared separately, following a kinetically controlled seeded-growth method previously reported (Bastus et al., 2014) with slight modifications. The approach is based on the reduction of silver nitrate $\left(\mathrm{AgNO}_{3}\right)$ in the presence of two competing reducing agents, tannic acid (TA) and trisodium citrate hexahydrate (SC) at $100^{\circ} \mathrm{C}$. Six different Ag NPs were synthesized by surface-coating both sizes with bovine serum albumin (BSA), chitosan (Chit) or polyvinylpyrrolidone (PVP) to generate negative Ag NP_BSA, positive Ag NP_Chit and neutral Ag NP_PVP, respectively. Details of these procedures are elaborated upon in the electronic supplementary material.

An aqueous suspension of a negatively charged $50 \mathrm{~nm}$ PVP-coated Ag NP (Ag NP_NC) was obtained from NanoComposix ${ }^{\circ}$ (San Diego, US) and included in this study for comparison with the synthesized Ag NPs. Based on the manufacturer's information, the Ag NP_NC is reported to have mean core- and hydrodynamic diameters of $54.8 \pm 10 \mathrm{~nm}$ and $72 \pm 14.4 \mathrm{~nm}$ respectively. The stock suspension of the pristine Ag NP has a mass concentration of $5.0 \mathrm{mg}$ $\mathrm{Ag} / \mathrm{mL}$, and a particle number concentration of $5.2 \times 10^{12}$ particles $/ \mathrm{mL}$. The surface area was $9.8 \mathrm{~m}^{2} / \mathrm{g}$ while surface charge (zeta-potential) was $-37.8 \mathrm{mV}$. The DLS and zeta potential in both milliQ and DMEM were confirmed in our laboratory, and details previously provided (Makama et al., 2015).

\section{Ag NP Characterization}

Prior to exposure experiments, pristine Ag NPs were dispersed in Dulbecco's modified Eagle's medium, DMEM (Invitrogen Breda, NL), as well as milliQ water and characterized by transmission electron microscopy (TEM), UV-Vis spectroscopy, dynamic light scattering (DLS), and zeta-potential measurements ( $\zeta$-potential). A combination of different techniques was used to characterize the Ag NPs and to monitor their proper coating and stability in the different media. Characterization details are given in the electronic supplementary material. 


\section{NPs dispersions}

Ag NPs stock dispersions were prepared by first suspending the respective powders in milliQ water except for Ag NP_Chit, where $50 \mathrm{mM}$ acetic acid (Merck, Darmstadt, DE) was used instead. Ag NP_Chit suspensions were initially prepared in $50 \mathrm{mM}$ acetic acid in order to prevent agglomeration while ensuring monodispersity (Zhao et al., 2011, Nadesh et al., 2013). Where used, the final acetic acid concentration in the medium at which the cells were exposed to was $4 \mathrm{mM}(0.02 \%)$, and determined to be non-cytotoxic (data not shown). For all Ag NPs, the stock dispersions were prepared at $2.5 \mathrm{mg} \mathrm{Ag} / \mathrm{mL}$ and sonicated in a Sonorex RK100 (Berlin, DE) water-bath over ice for $15 \mathrm{~min}$. Further dilutions to the desired exposure concentration ranges $(0.1,1,5,10,20,50,100$, and $200 \mu \mathrm{g} \mathrm{Ag} / \mathrm{mL})$ were made in DMEM fortified with heat inactivated fetal calf serum (FCS) at $10 \%$.

\section{Ag NPs exposure and Uptake dynamics}

A mouse monocyte macrophage cell line, RAW 264.7 (ATCC ${ }^{\circ}$ TIB-71"; Manassas, VA US) was used in this study at passages 16-22. Cells were cultured in DMEM, enriched with FCS at $10 \%(\mathrm{v} / \mathrm{v})$. Sub-culturing and passaging of cells was done every $3-4$ days when reaching $80 \%$ confluency by trypsinization after rinsing with phosphate buffered saline (PBS). Before exposure to Ag NPs, cells were grown for $24 \mathrm{~h}$ in 96 -well plates at a seeding density of $10^{5}$ cells/well for all assays, and incubated at $37^{\circ} \mathrm{C}$ in humidified air (plus $5 \% \mathrm{CO}_{2}$ ) to attain above $80 \%$ adherent confluency. After this initial $24 \mathrm{~h}$ of stabilization and growth, Ag NPs exposures for all assays with the exception of the Dichlorofluorescein (DCF) assay for ROS production, were conducted over a $24 \mathrm{~h}$ duration. For the ROS production assay, exposure to Ag NPs was for $6 \mathrm{~h}$, based on an adaptation of a similar method described earlier (Carlson et al., 2008). Unless stated otherwise, general incubation conditions were as described above.

Macrophages were imaged by means of confocal microscopy to follow cellular uptake, localization and accumulation behavior of the different Ag NPs over time. Imaging conditions were chosen such as to achieve low photon stress, without any visible loss of vitality after $24 \mathrm{~h}$ of exposure and imaging. This was performed for the $50 \mathrm{~nm}$ size class only, assuming that this large size is generally taken up less rapidly compared to $20 \mathrm{~nm}$ (Shang et al., 2014a, Shang et al., 2014b). Details on methodology and other results are provided in the electronic supplementary material.

\section{Effect assessment}

Cytotoxicity assays were performed to evaluate the viability of the macrophages after exposure to the different Ag NPs. 3-(4,5-dimethylthiazol-2-yl)-2,5-diphenyltetrazolium bromide (MTT) assay (Kloet et al., 2015); ATP content was determined using a bioluminescent somatic cell assay kit (Sigma-Aldrich', St Louis, MO, US), and; mitochondrial permeability tran- 
sition-pore (MPTP) assays were performed using a commercial kit from Molecular Probes (Leiden, NL). The productions of ROS as well as the induction of TNF- $\alpha$ were also assessed to elucidate specific modes of action involved. A 2',7'-dichlorodihydrofluorescein diacetate $\left(\mathrm{H}_{2} \mathrm{DCF}-\mathrm{DA}\right)$ dye from Molecular probes (Carlsbad, CA, US) was used for ROS, while TNF- $\alpha$ induction was assessed by a solid phase sandwich Enzyme-Linked Immuno-Sorbent Assay (ELISA) using a Mouse TNF- $\alpha$ ELISA kit (Invitrogen ${ }^{\mathrm{TM}}$, Paisley, UK). Protocols for these assessments were based on the manufacturers' instructions, and details are provided in the electronic supplementary material.

\section{Controls}

Macrophage cells exposed to DMEM, containing equal proportions of milliQ $\mathrm{H}_{2} \mathrm{O}$, but no NPs served as blanks. For exposures with Ag NP_Chit group, $0.02 \%$ acetic acid (4 mM final exposure concentration) was used. Positive controls were prepared in exposure medium without NPs and included for each assay. All controls were incubated according to the respective protocols and results can be found in the supplementary Fig. S1. To check the likelihood of particle interference with spectroscopic measurements, different Ag NP concentrations were included (in the absence of cells) and measured for all assays. Readings confirmed no interference of the particles with the fluorescence, absorbance or luminescence readings, and all controls were as expected (Supplementary Fig. S1).

\section{RESULTS}

\section{Ag NPs Characterization}

Fig. $1 \mathrm{a}-\mathrm{c}$ presents representative TEM images and UV-Vis spectra of $50 \mathrm{~nm}$ Ag NPs characterization, while information on the $20 \mathrm{~nm}$ size group is provided in supplementary Table S1 and Fig. S2. TEM images below (Fig. $1 \mathrm{a}-\mathrm{c}$ ) indicate visually that the primary particle sizes targeted by the synthesis were achieved and that for all coatings, the morphology of the particles were preserved after coating and lyophilization processes. Average particle sizes obtained by analysis of over 250 NPs by TEM were $51.1 \pm 5.7,51.9 \pm 6.4$ and 51.0 \pm 6.1 for Ag NP_BSA, Ag NP_Chit and Ag NP_PVP $50 \mathrm{~nm}$ size group, respectively (Fig. 2 a - c). For the $20 \mathrm{~nm}$ group, these values were respectively $19.5 \pm 5.4,18.2 \pm 5.1$ and $23.8 \pm 4.6 \mathrm{~nm}$.

The UV-Vis specter of pre-coated Ag NPs showed SPR peaks centered at $405 \mathrm{~nm}$ (Supplementary Fig. S2 d-f) for the $20 \mathrm{~nm}$ size group, and $441 \mathrm{~nm}$ (Fig. $1 \mathrm{~d}$-f dashed line) for the $50 \mathrm{~nm}$ sizes. Also, the hydrodynamic sizes of the $20 \mathrm{~nm}$ and $50 \mathrm{~nm} \mathrm{Ag} \mathrm{NPs} \mathrm{were} \mathrm{determined} \mathrm{to} \mathrm{be} 36$ $\mathrm{nm}$ and $55 \mathrm{~nm}$, respectively. Coating the Ag NPs with BSA, PVP and Chitosan led to a slight red-shift in the positions of the SPR peaks, indicating an affinity of these molecules for the silver surfaces and their spontaneous association. The SPR peaks shifted 6-8 $\mathrm{nm}$ in the case of Ag NP_BSA, 2-3 nm for the Ag NPs-PVP and 9-10 nm for the Ag NPs-Chit. 
DLS measurements in milliQ water and DMEM showed larger particles sizes (Supplementary Table S1) than those obtained from TEM. The hydrodynamic sizes for $20 \mathrm{~nm} / 50 \mathrm{~nm}$ NPs were $41.5 \pm 1.3 / 69.9 \pm 3.2$ and $46.8 \pm 0.1 / 71.3 \pm 2.0$ for Ag NP_BSA and Ag NP_PVP respectively. In accordance with these results, the hydrodynamic sizes of the Ag NPs after their surfaces were coated also increased by 10-12 nm for the Ag NPs_BSA, 15-20 nm for the Ag NPs_PVP and 50 - $100 \mathrm{~nm}$ for Ag NP_Chit. Ag NP_Chit formed agglomerates during re-suspension, consequently overestimating the average diameter to be around $240 \mathrm{~nm}$ for both sizes. Fig. 2 shows the particle size distribution of Ag NPs as determined by TEM. The frequency curves shifts from left to right with increasing particle sizes. The surface charges of the $20 \mathrm{~nm} \mathrm{Ag}$ NPs measured in water (and DMEM) showed $\zeta$-potentials (meV) of $-37.0 \pm 2.0(-23.0 \pm 2.0)$, $+7.0 \pm 1.0(-21.3 \pm 1.4)$ and $-22 \pm 2.0(-19.3 \pm 0.7)$ for Ag NP_BSA, Ag NP_Chit and Ag NP_PVP, respectively. The surface charges for the $20 \mathrm{~nm}$ particles were similar to their $50 \mathrm{~nm}$ counterparts (see supplementary material Table S1). After coating, the $\zeta$-potential measured indicated changes in the surface charges from -40 to $-50 \mathrm{meV}$ corresponding to citrate ions on the surface of the pre-coated intermediate product of Ag NP synthesis. These changes were between -29 and $-37 \mathrm{meV}$ (Ag NP_BSA), -16 and $-22 \mathrm{meV}$ (Ag NP_PVP) and +7 to $+8 \mathrm{meV}$ (Ag NP_Chit). The $\zeta$-potential values in DMEM were all negative, although the negatively charged Ag NP_BSA had a slightly more negative potential in all measurements. 

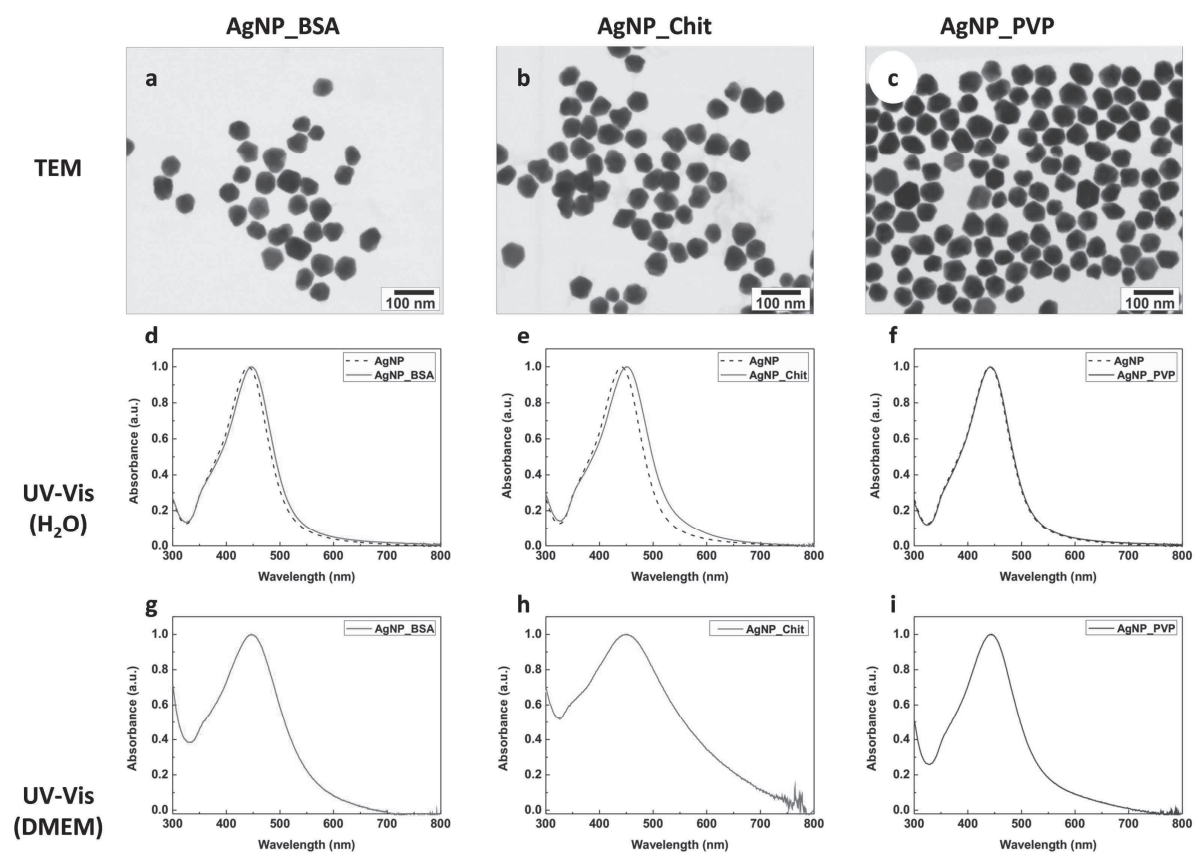

Figure 1. Representative Ag NPs characterization results for $50 \mathrm{~nm}$ size group. TEM images (a - c) of re-suspended Ag NPs showing quasi-spherical particles within the expected size ranges, with overall average diameter of $51.3 \pm 6.1 \mathrm{~nm}$. UV-Vis also shows SPR peaks within the expected wavelength range $300-600 \mathrm{~nm}$ both in milliQ water and in cell culture medium ( $\mathrm{d}-\mathrm{f}$ and $\mathrm{g}-\mathrm{i}$, respectively). The UV-Vis SPR curves of coated and non-coated Ag NPs nicely overlaps ( $-\mathrm{f}$ ), with the post-coating SPR curves exhibiting a red-shift indicative of the effect of Ag NPs coating. DMEM: Dulbecco's modified Eagle's medium.

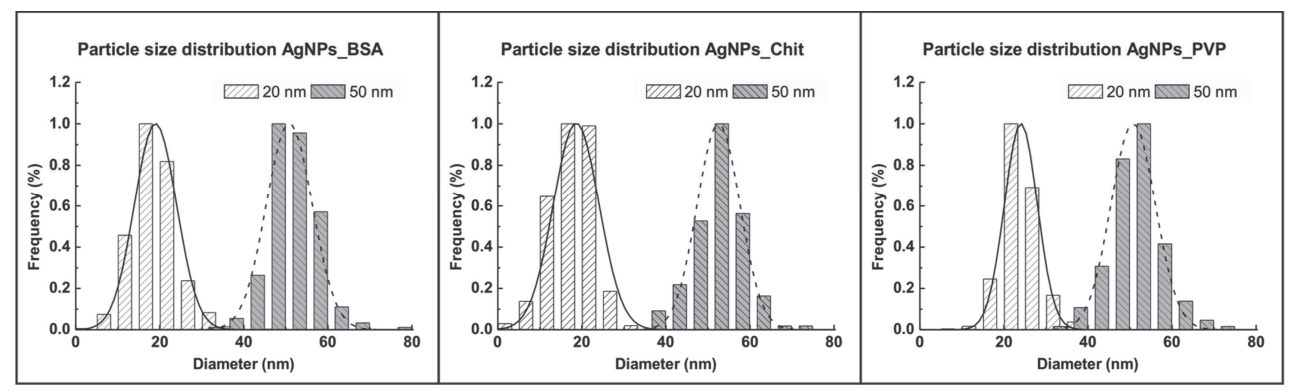

Figure 2. Particle size distribution of Ag NP_BSA (a), Ag NP_Chit (b) \& Ag NP_PVP (c) showing frequency counts as determined by transmission electron microscopy (TEM). The frequency curves shifts from left to right with increasing particle sizes: $20 \mathrm{~nm}$ (solid lines); $50 \mathrm{~nm}$ (broken lines). 


\section{Confocal localization and uptake dynamics of Ag NPs}

Fig. 3 shows a time series of macrophages imaged in $t z$ intervals for $2.5 \mathrm{~h}$ during exposure to 5 $\mu \mathrm{M}$ Ag NP_BSA, indicating Ag NPs as red dense regions in the cytoplasm of cells. Additional imaging results are provided in the electronic supplementary material.

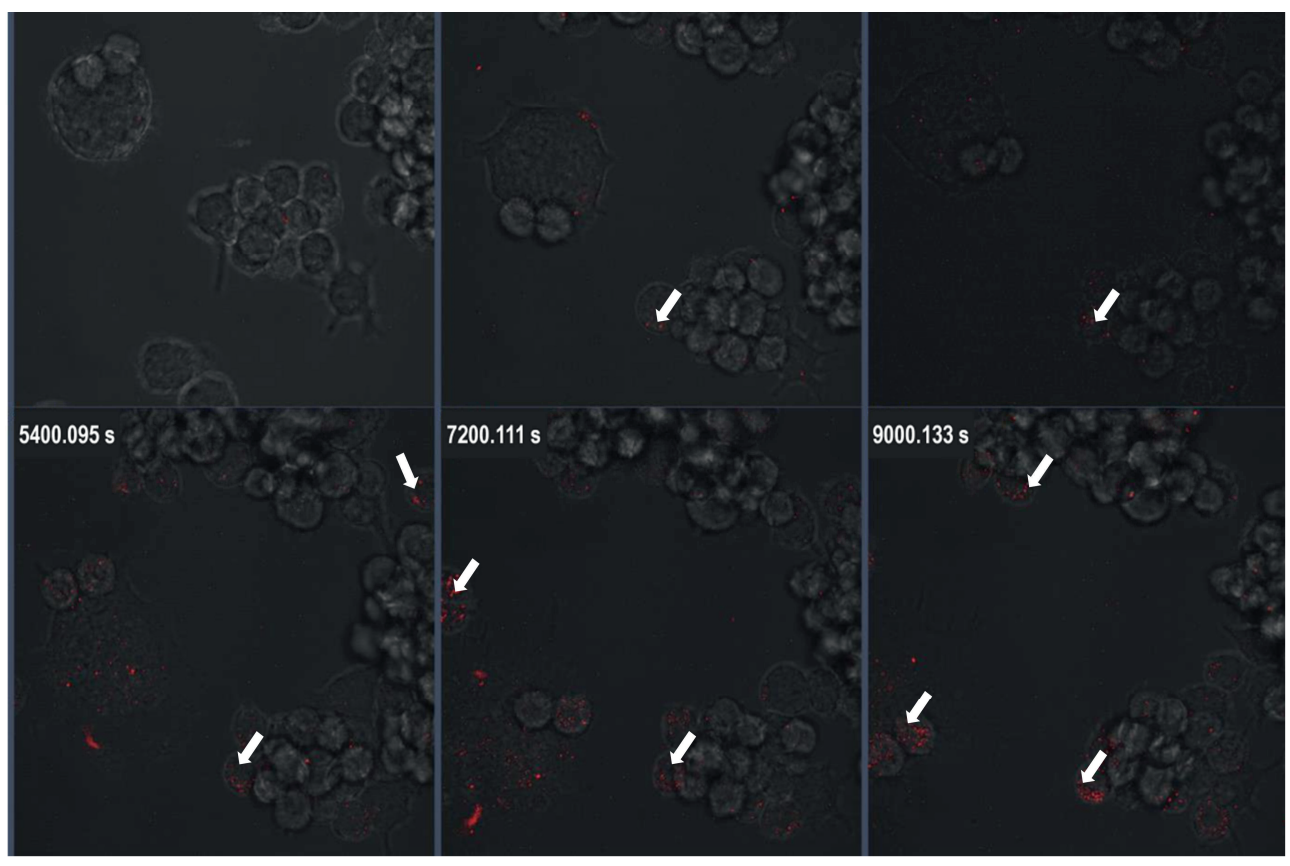

Figure 3. Frames of a time series of macrophages imaged in $t z$ intervals for $2.5 \mathrm{~h}$ during exposure to 5 $\mu \mathrm{M}$ Ag NP_BSA. Maximal intensity $z$-projections were made of optical sections in the 7-14 $\mu \mathrm{m}$ midrange, representing the maximal fluorescent signal of individual time-points. Ag NPs are visible as red dotted regions in the cytoplasm of cells (white arrows), while the non-confocal bright field image is displayed in grey levels (BW). Displaced clusters show how cells move in the $x y$ plane over time (0-9000 sec). These frames were captured from the supplemental movie MOV_Ag NPs.avi.

\section{Effect assessments}

\section{MTT Assay}

There was a concentration-dependent decrease in mitochondrial function assessed by the MTT viability assay, with smaller Ag NPs $(20 \mathrm{~nm})$ presenting lower $\mathrm{EC}_{50}$ values compared to their larger $(50 \mathrm{~nm}$ ) counterparts (Fig. $4 \mathrm{a}-\mathrm{d}$; Table 1). Exposure of the cells to the Ag NP_BSA results in a statistically significant decrease in MTT, already at low exposure concentration of $10 \mu \mathrm{g} / \mathrm{mL}$ for the $20 \mathrm{~nm}$ Ag NPs. Further statistical analyses however, did not 
present any significant difference between the $\mathrm{EC}_{50}$ values obtained for the different sizes (20 $\mathrm{nm}$ vs $50 \mathrm{~nm}$ ) nor types (surface-coating/charge) of Ag NPs. Except for Ag NP_Chit $50 \mathrm{~nm}$ for which the $\mathrm{EC}_{50}$ value exceeded the highest exposure concentration (Table 1).

Table 1. Table of $\mathrm{EC}_{50} \mathrm{~s}(\mu \mathrm{g} / \mathrm{mL})$ for assays, representing the effective concentration of Ag NPs required to produce the effect (increase/decrease) by $50 \%$.

\begin{tabular}{lccccccccccc}
\hline & \multicolumn{3}{c}{ Ag NP_BSA } & \multicolumn{3}{c}{ Ag NP_Chit } & \multicolumn{3}{c}{ Ag NP_PVP } & \multicolumn{2}{c}{ Ag NP_NC } \\
Assay & $20 \mathrm{~nm}$ & $50 \mathrm{~nm}$ & P-value & $20 \mathrm{~nm}$ & $50 \mathrm{~nm}$ & P-value & $20 \mathrm{~nm}$ & $50 \mathrm{~nm}$ & P-value & $50 \mathrm{~nm}$ \\
\hline MTT & 11.7 & 58.9 & 0.224 & 10.3 & 125.9 & 0.553 & 14.8 & 37.6 & 0.438 & 40.7 \\
ATP & 28.4 & 114.7 & 0.005 & 93.0 & $>200$ & 0.006 & 66.8 & 104.2 & 0.333 & 182.0 \\
MPTP & 23.3 & 114.3 & 0.197 & 14.5 & 24.3 & 0.112 & 56.3 & 109.2 & 0.301 & 137.3 \\
ROS & 77.6 & 31.6 & 0.073 & $>200$ & $>200$ & $<0.001$ & 80.2 & $>200$ & 0.588 & 89.5 \\
TNF- $\alpha$ & 1.1 & 5.0 & 0.156 & 49.9 & 15.1 & 0.002 & nc & 14.2 & 0.038 & 93.6 \\
\hline
\end{tabular}

Significant when $\mathrm{p}<0.05$; nc, not converged.

\section{Mitochondrial permeability transition pore (MPTP) Assay}

Results indicated a significant opening of the MPTP with increasing Ag NP exposure concentration. A slight right-shift of the Ag NPs $50 \mathrm{~nm}$ dose-response curves was observed when compared to the curves obtained after exposure to the $20 \mathrm{~nm} \mathrm{Ag} \mathrm{NPs} \mathrm{(Fig.} 4 \mathrm{e}-\mathrm{h}$ ). Data showed that higher exposure concentrations of the $50 \mathrm{~nm}$ Ag NPs were needed to elicit the same loss in MPTP integrity as was obtained with the $20 \mathrm{~nm} \mathrm{Ag} \mathrm{NPs,} \mathrm{indicating} \mathrm{a} \mathrm{more} \mathrm{ef-}$ ficient induction of opening of the MPTP by the smaller Ag NPs. These differences however were not statistically significant. The $\mathrm{EC}_{50} \mathrm{~s}$ for the 20 and $50 \mathrm{~nm}$ sizes were only within 1-fold difference for chitosan- and PVP-coated NPs (Table 1). The $\mathrm{EC}_{50}$ for the $50 \mathrm{~nm} \mathrm{Ag} \mathrm{NP \_ BSA}$ exceeded the highest exposure concentration of $200 \mu \mathrm{g} / \mathrm{mL}$.

\section{Adenosine Triphosphate (ATP) Assay}

With increasing exposure concentration, the amount of ATP measured in the cells decreased in a concentration dependent manner, suggesting a loss in viability since ATP is rapidly degraded or lost from the cells as they die (Eguchi et al., 1997). For Ag NP_Chit and Ag NP_ $\mathrm{BSA}$, there was a significant effect of size in addition to exposure concentration, with $p$ values of 0.006 and 0.005 respectively when comparing the $\mathrm{EC}_{50} \mathrm{~s}$ for the respective 20 and $50 \mathrm{~nm}$ particles. There was no effect of size for the Ag NP_PVP. Similar to the $50 \mathrm{~nm} \mathrm{Ag} \mathrm{NP \_ PVP,} \mathrm{Ag}$ NP_NC (also $50 \mathrm{~nm}$, PVP-coated, but negatively charged), ATP was significantly depleted at the highest exposure concentrations, and $\mathrm{EC}_{50}$ values for both types of Ag NPs exceeded the highest concentration of exposure (Fig. 4 i - l; Table 1). 


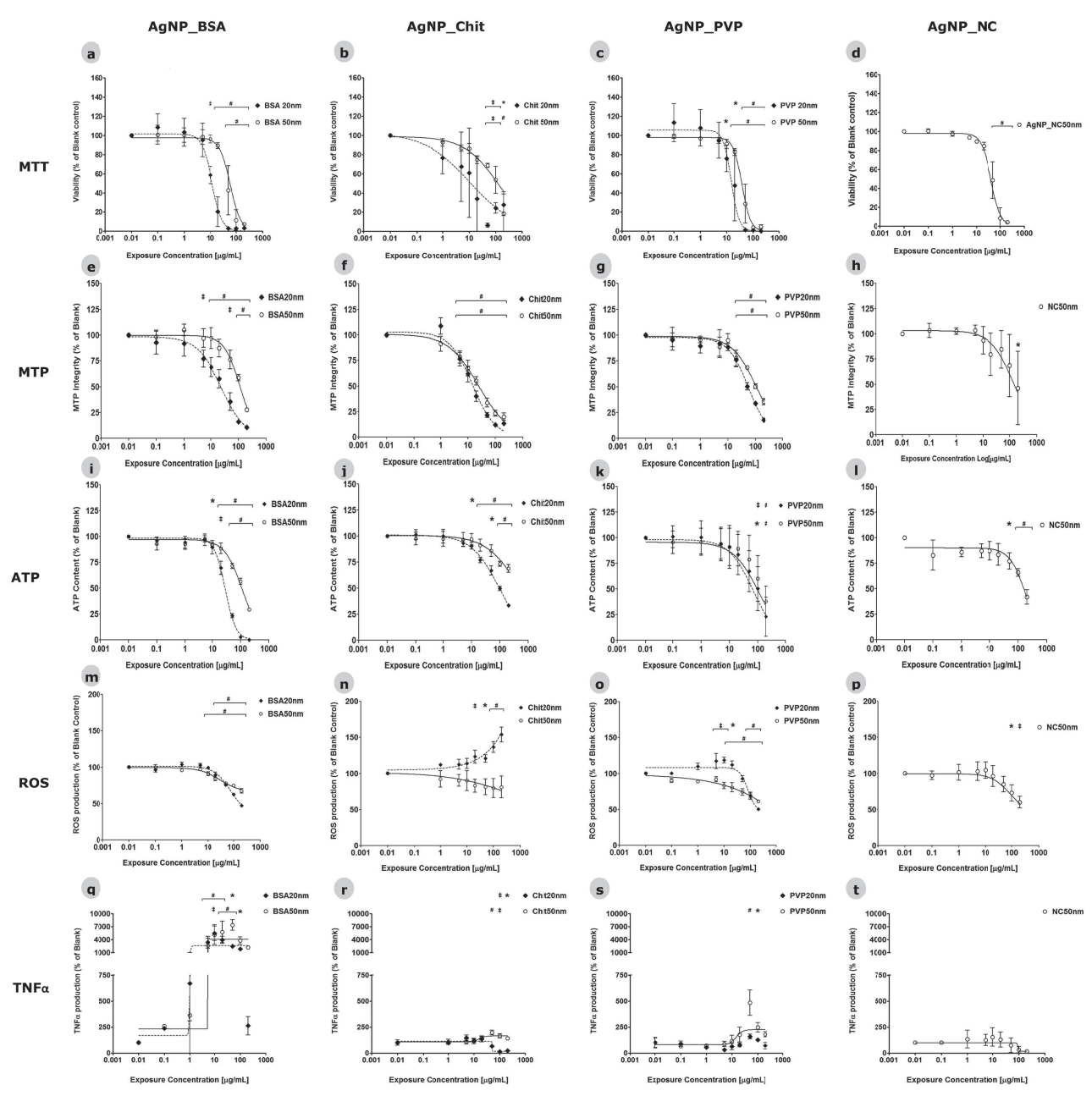

Figure 4. Representative Cytotoxicity of 20 and $50 \mathrm{~nm}$ NPs (Ag NP_BSA, Ag NP_Chit, Ag NP_PVP) and $50 \mathrm{~nm}$ Ag NP_NC to mouse monocyte macrophage cells (RAW 264.7 cell line) assessed by MTT $(\mathrm{a}-\mathrm{d})$, MPTP $(\mathrm{e}-\mathrm{h})$ and ATP $(\mathrm{i}-\mathrm{l})$ assays; and by assays for reactive oxygen species (ROS) production $(\mathrm{m}-\mathrm{p})$ and TNF- $\alpha$ production $(\mathrm{q}-\mathrm{t})$. Results are expressed as percentages (mean \pm standard deviation, $\mathrm{n}=3$ ) relative to the blank, set at $100 \%$. Dotted lines and filled diamond $(20 \mathrm{~nm})$; solid lines and empty circle $(50 \mathrm{~nm})$. Statistical significance compared to control is indicated by ${ }^{*}(\mathrm{p} \leq 0.05) ; \ddagger(\mathrm{p} \leq 0.01) ; \#(\mathrm{p} \leq$ 0.001). Cytotoxicity (MTT, ATP and MPTP assays) showed a similar pattern for all tested Ag NPs, with viability decreasing with increasing exposure concentrations and minimal differences between NPs of different sizes. On mechanisms of action (ROS production and TNF- $\alpha$ induction) however, there were effects of NP type and size (ROS production, 3n), while surface coating of Ag NPs with BSA appeared to result in a potent TNF- $\alpha$ induction (3q). 


\section{Intracellular Reactive Oxygen Species (ROS) generation; $\mathrm{H}_{2}$ DCF-DA Assay}

Ag NP_Chit $20 \mathrm{~nm}$ were the only NPs observed to increase ROS after $6 \mathrm{~h}$ exposure (Fig. $4 \mathrm{~m}$ p). For these NPs, a 1.5x increase in ROS was observed at the highest exposure concentration (Fig. 4 n). The "uncharged" Ag NP_PVP 20 nm, also showed an initial increase in ROS until $10 \mu \mathrm{g} / \mathrm{mL}$ beyond which the curve steepens downwards. For the $50 \mathrm{~nm}$ NPs, no increase in ROS was observed for any type of Ag NPs tested (Fig. $4 \mathrm{~m}-\mathrm{p}$ ). Estimates of the $\mathrm{EC}_{50} \mathrm{~s}$ indicated lower values for the $20 \mathrm{~nm}$ size Ag NPs, and only the Ag NP_Chit showed significant differences between the two sizes tested (Fig. 4 n). Comparing the different $20 \mathrm{~nm} \mathrm{Ag} \mathrm{NPs,}$ the $\mathrm{EC}_{50}$ estimated for ROS generation for Ag NP_Chit was significantly higher than those of the other types (Fig. $4 \mathrm{n}$ ).

\section{TNF- $\alpha$ induction}

Ag NP_BSA were most potent in inducing TNF- $\alpha$, presenting marked increase relative to the control (Fig. 4 q). The other NP types initially appeared to induce some TNF- $\alpha$, but this dropped below the reference (control) with increasing concentration, coinciding with the onset of cytotoxicity (Fig. $4 \mathrm{q}-\mathrm{t}$ ). An opposite size-dependent effect with chitosan- and PVP-coated Ag NPs can be noted, where some marginal induction of TNF- $\alpha$ was shown more by the $50 \mathrm{~nm}$ than the $20 \mathrm{~nm}$ Ag NPs (Fig. $4 \mathrm{~s}$ ). An interesting observation was made with the two different types of PVP-coated Ag NPs, where for TNF- $\alpha$ induction they gave different results (Fig. $4 \mathrm{~s}$ and $\mathrm{t}$ ) even though for all other assays they behaved similarly (Fig. 4). Also, there were significant differences in effects produced by the different sizes of Ag NP_Chit and Ag NP_PVP ( $p=0.002$ and 0.038 respectively), though TNF- $\alpha$ induction was relatively low (1.5 - 2 fold increase) compared to the induction observed with Ag NP_BSA (Fig. $4 \mathrm{q}-\mathrm{t}$ ).

\section{DISCUSSION}

In this present study, we focused on two very important properties; size and surface coating/ charge, identified from literature to be important in influencing the outcome of exposure to NPs. Here we present a more appropriate comparison of the influence of these NP properties by using synthesized Ag NPs from the same stock, designed to differ at specific properties of interest. Our study showed similar cytotoxicity patterns for all tested Ag NPs, differing only at specific modes of action. Additionally, we present further evidence of the cellular uptake dynamics of different Ag NPs.

\section{NP Synthesis, Dispersion and Characterization}

By varying the reaction parameters (temperature, seeding concentration and amount of precursor added), the desired Ag NP size ranges 20 and $50 \mathrm{~nm}$ were achieved. The average NP sizes as determined by TEM were found to be within expected ranges, and depending on the surface coating used, negative, positive and "neutral" charges were obtained. While coating 
with BSA and PVP is spontaneous and easy to monitor by the characterization techniques described above, that with chitosan resulted in Ag NPs aggregation. This may likely be due to the interaction of the negatively charged citrate Ag NP, an intermediate/pre-coated product of Ag NPs synthesis, with the positively charged chitosan (Gnanadhas et al., 2013, Bastus et al., 2014). To overcome this effect, a fast mixing of relatively small volumes of Ag NPs and an excess amounts of chitosan was used, resulting in a fast coating of the NPs.

Before coating, the particles showed SPR peaks and hydrodynamic sizes in accordance with expected results for the three different sizes (Bastus et al., 2014). A slight shift in the SPR peak was observed by UV-Vis spectroscopy after incubating the NPs for $24 \mathrm{~h}$ with the respective coating molecules. This shift is related to modifications in the close environment of the NPs and proves the affinity of the BSA, PVP and chitosan molecules for the silver surfaces and their spontaneous association (Bastus et al., 2014). The shape of the SPR peaks were preserved in both milliQ water and DMEM (Fig. $1 \mathrm{~d}-\mathrm{f}$ and $\mathrm{g}-\mathrm{i}$, respectively), which means that the particles were dispersed and stable under these conditions. The DLS measurements in both milliQ water and DMEM however, systematically resulted in larger sizes than those obtained before lyophilization. This could be indicative of a few agglomerates formed during the re-suspension of the powdered Ag NPs. Despite the large hydrodynamic diameter seen in the particles coated with chitosan, we observe well dispersed particles by TEM (Fig. 1 b).

Dispersed Ag NP_Chit formed some aggregates in the suspensions media, and these may have led to an overestimation of the hydrodynamic sizes during the DLS measurements, resulting in rather high NP diameters (Supplementary Table S1). Because the DLS operates on Rayleigh's approximation principle of light scattering by particles where the intensity of scattering is proportional to the sixth power of the particle's radius, DLS technique is very sensitive to particle agglomerates and/or any aggregation in NP suspensions (Hagendorfer et al., 2012). Hence, the occurrence of even minute amounts of agglomerates may skew the results. This aggregation observed with Ag NP_Chit for instance, represents a small fraction of the NPs, otherwise the SPR peaks would not be preserved (Fig. $1 \mathrm{~d}-\mathrm{i}$ ), and it would also be noticeable in the TEM images. Hence, the results indicate the formation of some aggregates but the majority of the Ag NPs appear to be single NPs, even for Ag NP_Chit.

Following dispersion in DMEM, the surface charges changed to negative values in all Ag NPs. Positively charged particles were likely coated by negatively charged proteins in the media, and may explain the negative $\zeta$-potentials measured for all Ag NPs. A Z-potential measurement in a medium like DMEM containing an abundance of charged protein molecules is greatly influenced by the electrostatic interactions in the matrix. This was demonstrated by others (Pavlin and Bregar, 2012, Shannahan et al., 2013). Similarly, we observed more negative $\zeta$-potentials values (Supplementary Table S1) when Ag NPs were re-suspended in water, 
with minimal effect on hydrodynamic sizes regardless of the dispersant used. Lyophilization of the samples did not result in particle aggregation or surface modifications, and except for Ag NP_Chit, they could be easily dispersed in water as TEM images above show (Fig. $1 \mathrm{a}-\mathrm{c}$ ) and DMEM. The SPR peaks and the surface charges were preserved as well.

\section{Ag NPs localization and Uptake dynamics}

Uptake of the negatively charged Ag NP_BSA was rapid and most significant based on an arbitrary unit (AU) of quantification. Due to agglomeration in the suspension and growth medium, it was not possible to assess uptake of Ag NP_Chit by the cells. Supplementary Table S2 shows that the mean cellular uptake of Ag NP_BSA was twice that of Ag NP_PVP at similar exposure concentrations and time, though the uptake efficiency of individual cells does vary a lot. Several cells showed fast uptake, with up to $80 \%$ of the Ag NPs observed to be taken up within the first $30 \mathrm{~min}$ of exposure (not shown). After $2.5 \mathrm{~h}$ however, cytoplasmic fluorescence intensity levels reached a plateau for the different Ag NPs tested (Table S2). In fact, there was no significant increase in the mean fluorescent intensity in the macrophages after $24 \mathrm{~h}$ exposure (Supplementary Table S2). This rapid Ag NP uptake indicates that the $6 \mathrm{~h}$ exposure duration used for the assessment of ROS production is sufficient for cells to internalize the Ag NPs. With increasing exposure concentrations, the mean AU plateau value increased nonlinearly for all Ag NPs. Fresh re-exposure at the same concentration and for an additional $24 \mathrm{~h}$ did not significantly increase the plateau value either. Even at the lower exposure ranges, it can be seen that the uptake after $2 \mathrm{~h}$ is already reaching a plateau for the different Ag NPs (Table S2) and this may suggest that cells loose their uptake capacity over time.

Cellular vitality was monitored during uptake assays by intercellular dynamics and presence of membrane protrusions, the latter being typical for functional macrophages (Vonna et al., 2007, Venter et al., 2014). Over a period of $48 \mathrm{~h}$, no apparent cell death or abnormalities were observed while visualizing the subcellular bio-distribution of the Ag NPs. Subcellular dynamics remained visible during standardized imaging, as well as after $48 \mathrm{~h}$ exposure to $5 \mu \mathrm{g} / \mathrm{mL}$ of $50 \mathrm{~nm}$ Ag NP_BSA (Supplementary Fig. S4). The density of membrane protrusions however, slowly decreased over time $(2.5-24 \mathrm{~h}$, not quantified). Only after prolonged local imaging were loss of membrane protrusions, amorphic membrane blebbing and cell fusion events triggered, indicating photon stress. We also demonstrated the uptake dynamics of Ag NP_BSA sticking to a macrophage surface membrane protrusion, and consequently being transported to the cell body within 30 seconds. The time series shows the dynamics of individual vesicles of various sizes in the cytoplasm (Fig. 3 and MOV_Ag NPs.avi). Both dispersed small vesicles as well as vesicles with clustered accumulated Ag NPs, occurred for all Ag NPs. We cannot exclude the likelihood that free Ag NPs were also present in the cytoplasm (Supplementary Fig. S4). Over time, more clustered vesicles appeared, indicating vesicle fusion. Multiple large 
endosomes or lysosomes with many Ag NPs clustered together were observed, suggesting that uptake was not by diffusion. There are different types of active endocytotic pathways such as receptor mediated endocytosis (clathrin or caveolin mediated) and macropinocytosis, but the current study did not discriminate between those.

Vesicle density varied over the cytoplasmic domain, but Ag NPs were never observed in the nucleoplasms (Fig. 3 and Supplementary Fig. S4). The absence of $50 \mathrm{~nm} \mathrm{Ag} \mathrm{NPs} \mathrm{in} \mathrm{the} \mathrm{nuclei}$ was expected since nuclear pores have an effective diameter of $9-10 \mathrm{~nm}$. After $24 \mathrm{~h}$ loading, cells were still viable and healthy as judged by the dynamics from the cells themselves as well as from the organelles within. The dynamic movements of the Ag NPs within membrane domains indicate that accumulation does not imply clustering (MOV_Ag NPs.avi).

\section{Effect Assessment}

The smaller $20 \mathrm{~nm}$ Ag NPs tend to induce earlier effects than the $50 \mathrm{~nm}$ sizes in almost all assays reported here (Fig. 4 and Table 1). However, the differences were minimal and for all Ag NPs tested, MTT reduction and MPTP opening did not differ significantly $(p<0.05)$ between different sizes (20 nm vs $50 \mathrm{~nm}$ ) and surface-coatings or charges. A concentration dependent decrease in MTT reduction was observed, with the $20 \mathrm{~nm}$ NPs showing lower $\mathrm{EC}_{50}$ values than the $50 \mathrm{~nm}$ NPs (Fig. 4 and Table 1). Ag NP_BSA NPs appear to elicit the most effect. With the exception of Ag NP_Chit $50 \mathrm{~nm}$ where the $\mathrm{EC}_{50}$ exceeded the highest exposure concentration, $\mathrm{EC}_{50} \mathrm{~s}$ were between 10 and $15 \mu \mathrm{g} / \mathrm{mL}$ and 35 and $40 \mu \mathrm{g} / \mathrm{mL}$ for the $20 \mathrm{~nm}$ and 50 $\mathrm{nm}$ size groups respectively (Table 1 ). The influence of NP size on its ability to induce toxic effects has been demonstrated previously, (Liu et al., 2010, Park et al., 2011) for instance. Our findings partially agree with the proposition that size does matter as could be seen with ATP production, where the $20 \mathrm{~nm}$ Ag NPs were more potent than the $50 \mathrm{~nm}$ for both Ag NP_BSA and Ag NP_Chit (Fig. 4 i - l; Table 1).

ATP assay results indicated a significant decrease at low exposure concentrations of $10 \mu \mathrm{g} /$ $\mathrm{mL}$, at which the mitochondrial respiratory systems were also impaired (Fig. 4). This effect may likely be due to mitochondrial damage (AshaRani et al., 2009). Interestingly and contrary to the cited report in which ATP depletion in the tested cells was observed only after $48 \mathrm{~h}$, ATP was depleted after $24 \mathrm{~h}$ exposure in our current study. The surface coating used for the Ag NPs in the cited study was starch, and may have interacted differently with the cells, potentially delaying the onset of toxicity. We found an increased opening of the mitochondrial transition pore with increasing exposure concentration, leading to a rapid change in permeability. This is likely followed by membrane depolarization, release of intra-mitochondrial ions and metabolic intermediates amongst other effects (Lemasters et al., 1998). Normally, the MPTP remains closed unless under conditions of stress as found in for instance hypoxia, oxidative stress, and exposure to a calcium ionophore. Ag NPs of all sizes and surface coating tested 
demonstrated ability to cause the MPTP to open, leading to the quenching of the signal from the calcein which is now accessible to the $\mathrm{CoCl}_{2}$. In our study however, we could not demonstrate ROS production for most Ag NPs tested (Fig. 4 m-p). Thus, other factors including increased accumulation of intracellular fatty acids and lysophosphatidase, as well as glutathione oxidation (Lemasters et al., 1998), may also be responsible for activating MPTP opening.

Intracellular ROS generation is an outcome of normal cellular metabolism and these radicals may be cleared by the cell's scavenging processes. Increase in ROS production however, is considered to be an early phase response to toxicants (Aranda et al., 2013), and has been reported as one of the likely mechanisms of toxicity following exposure to Ag NPs as well as other NPs (Asharani et al., 2011, Foldbjerg et al., 2011, Jeong et al., 2011). In this current study, ROS production was only slightly increased with $20 \mathrm{~nm} \mathrm{Ag} \mathrm{NP \_ Chit} \mathrm{which} \mathrm{showed} \mathrm{a}$ 1.5 times increase in ROS relative to the control at the highest exposure concentration (Fig. 4 n). Also, $20 \mathrm{~nm}$ Ag NP_PVP showed an initial increase in ROS, then a decrease as exposure concentration increases. This decrease in ROS production is associated with the onset of cytotoxicity (Fig. 4), and perhaps other cytotoxic processes are initiated or progressing faster than ROS generation. For example, the opening of the MPTP may lead to partial mitochondrial depolarization, which depending on $\mathrm{Ca}^{2+}$ concentrations, could result in a decrease in ROS production (low $\mathrm{Ca}^{2+}$ ) or an increase (high $\mathrm{Ca}^{2+}$ ) as reported earlier (Akopova et al., 2011).

The initial increase in ROS generation at lower exposure concentrations (Fig. $4 \mathrm{~m}$-p) agrees with the findings of others where it was suggested that cytotoxic effects were as a result of ROS production, particularly at low exposure concentrations and short incubation times (Carlson et al., 2008, Suresh et al., 2012, Foldbjerg et al., 2015). It may be that the cytotoxic effect of the Ag NPs overwhelms the cell's capacity to generate ROS as a response, and rather progresses to cell death following other routes as suggested above. Again, positively charged NPs tend to interact more readily with cells due to the negative cell membrane charges (Nafee et al., 2009). This may enhance the exposure of cells to positively charged NPs, likely explaining the increased ROS production observed with the $20 \mathrm{~nm}$ positively charged Ag NP_Chit (Fig. 4n) where there was a significant difference between the two sizes tested $(p<0.001)$. Interestingly, ROS was rather decreased in macrophage cells with increasing exposure concentrations of the $50 \mathrm{~nm}$ size Ag NP_Chit, similar to the other Ag NP types tested. With increasing exposure concentrations, the resulting distortion in metabolic activity coupled with an open MPTP, may eventually lead to ATP depletion and cell death. This process has also been associated with mitochondrial respiratory system impairment following oxidative stress (AshaRani et al., 2009). In this current study, ROS production appears to have been inhibited as Ag NPs exposure concentration increases relative to the control. Considering the early onset of cytotoxicity based on MTT, MPTP and ATP assay results however, the downward plunge of the ROS curves could result from the inability of injured cells to produce oxidative radicals, since their viability has been impaired. 
Ag NPs have been reported to cause immunogenic response in cells, characterized by induction of cytokines like TNF- $\alpha$, macrophage inhibitory protein, interleukins, etc. (Carlson et al., 2008, Park et al., 2011). For the Ag NPs tested in this current study, the negatively charged Ag NP_BSA showed the most induction with both sizes resulting in an 80 -fold increase between exposure concentrations 10 and $50 \mu \mathrm{g} / \mathrm{mL}$. Higher concentrations showed a downward plunge of the curves below the reference (control), coinciding with cytotoxicity (Fig. 4 a, e, $i$, and q). There were no significant differences in the effects induced by the different sizes of Ag NP_BSA (Fig. 4 q). Interestingly, even when coated with similar biomolecules, the behavior of NPs may still differ as can be seen with the $50 \mathrm{~nm}$ PVP-coated Ag NPs commercially produced (negatively charged) and the synthesized one (uncharged). Although both behaved similarly for all other assays tested, the case was different with TNF- $\alpha$ induction (Figs. $3 \mathrm{~s}$ and t) where Ag NP_PVP was a significantly higher TNF- $\alpha$ inducer than Ag NP_NC ( $p=$ 0.002). This could likely be due to the type of synthesis procedure applied for making not only the NPs (Powers et al., 2011a, Samberg et al., 2011), but also the bioconjugate (Bastus et al., 2014). The type or composition of the coating agent influences the outcome of exposure, corroborating previous findings (Aadil et al., 2016) where the influence of lignin concentration, incubation temperature and time in the synthesis of Ag NPs were assessed. Also, the $\mathrm{pH}$ of the solution during the synthesis was important in determining the surface charge of the NPs, with acidic $\mathrm{pH}$ resulting in negatively charged NPs while basic $\mathrm{pH}$ leads to positively charged NPs. This phenomenon is important not only during synthesis of NPs, but also during exposure (Deng et al., 2016).

The greater the NP's relative surface area, the greater the potential for reactivity. Since the relative surface area is determined by the particle size, smaller NPs may interact more with the macrophages (Johnston et al., 2010, Reidy et al., 2013) when administered on an equal mass-basis as was done in the current study. Moreover, surface coatings and their interactions with biomolecules can influence dissolution rates of Ag NPs (Yen et al., 2009, Yang et al., 2012), leading to toxicities. Based on this, one could hypothesize that the synthesized Ag NPs tested in this study were quite stable under the experimental conditions reported here. Longer exposure durations of $48-72 \mathrm{~h}$ may likely present different outcomes.

Also, some studies have shown an opposite effect of size for e.g. with PVP-coated Ag NP (Powers et al., 2011a) where the larger particles produced larger effect. In the present study, this was observed with Ag NP_Chit and Ag NP_PVP in TNF- $\alpha$ induction assay, with the 50 $\mathrm{nm}$ sizes having lower $\mathrm{EC}_{50}$ values (Table 1). For the tested Ag NPs in this study, cytotoxicity patterns were similar irrespective of types and size of the Ag NPs. Looking at ROS and TNF- $\alpha$ induction on the other hand revealed differences between the different types of Ag NPs. This is indicative of varying mechanisms of action involved, corroborating what has been opined by others (Johnston et al., 2010, Reidy et al., 2013). These authors suggested that a combina- 
tion of different physicochemical properties of Ag NPs is more likely responsible in inducing the observed toxicities, rather than only size or particle dissolution for instance, as the release of ions would be expected to be greater for smaller particles.

\section{CONCLUSION}

Our study used well established in vitro assays to investigate the influence of the physicochemical properties of a specifically designed set of different types of Ag NPs systematically synthesized to vary the properties of interest. The role of surface coating/charge in influencing TNF- $\alpha$ induction was demonstrated by the negatively charged Ag NP_BSA. Effect of size was less prominent under our experimental conditions, showing mostly minimal differences that were not statistically significant. Overall, the negatively charged Ag NP_BSA appears to be more potent in inducing adverse effects on the macrophages. The complexity in determining the fate and toxicity of Ag NPs (also other NPs) using in vitro or in vivo models is as yet a challenge that needs further evaluation. Live confocal imaging of exposed cells allowed the monitoring of Ag NPs uptake dynamics and subcellular cytoplasmic accumulation.

A combination of several factors likely play a role in determining the outcome of exposure. These include the processes involved in the NP syntheses, presence and type of coating agents resulting in various physicochemical properties (size, charge, hydrophobicity, etc.), as well as the toxicity tests models used and end-points studied. This has been observed in earlier reviews (Johnston et al., 2010, de Lima et al., 2012, Reidy et al., 2013), and still remains a hindrance for conclusive risk assessment evaluation of Ag NPs. Taken together, the results from MTT, ATP and MPTP assays shows that adverse effects from exposure to the tested Ag NPs were similar irrespective of type or size. However, for ROS and TNF- $\alpha$ induction, we do find significant toxicity differences with variations in the physicochemical properties of the Ag NPs tested.

\section{ACKNOWLEDGEMENTS}

This work was financially supported by NanoNextNL, a micro- and nanotechnology consortium of the Government of The Netherlands and 130 partners; funding was also received from Managing Risks of Nanoparticles, MARINA (EU-FP7, contract CP-FP 263215), and; the Strategic Research Funds titled Novel technologies by the Ministry of Economic Affairs of The Netherlands. Synthesis and characterization of the Ag NPs used in this study received support from the QualityNano Project http://www.qualitynano.eu/ which is financed by the European Community Research Infrastructures under the FP7 Capacities Program (Grant No. INFRA-2010-262163). 


\section{REFERENCES}

Aadil, K.R., Barapatre, A., Meena, A.S. \& Jha, H., 2016. Hydrogen peroxide sensing and cytotoxicity activity of Acacia lignin stabilized silver nanoparticles. Int J Biol Macromol, 82, 39-47.

Abou El-Nour, K.M.M., Eftaiha, A.A., Al-Warthan, A. \& Ammar, R.a.A., 2010. Synthesis and applications of silver nanoparticles. Arabian Journal of Chemistry, 3, 135-140.

Ahamed, M., Alsalhi, M.S. \& Siddiqui, M.K., 2010. Silver nanoparticle applications and human health. Clin Chim Acta, 411, 1841-8.

Akopova, O.V., Kolchynskayia, L.Y., Nosar, V.Y., Smyrnov, A.N., Malisheva, M.K., Man’kovskaia, Y.N. \& Sahach, V.F., 2011. The effect of permeability transition pore opening on reactive oxygen species production in rat brain mitochondria. Ukr Biokhim Zh (1999), 83, 46-55.

Aranda, A., Sequedo, L., Tolosa, L., Quintas, G., Burello, E., Castell, J.V. \& Gombau, L., 2013. Dichloro-dihydro-fluorescein diacetate (DCFH-DA) assay: a quantitative method for oxidative stress assessment of nanoparticle-treated cells. Toxicol In Vitro, 27, 954-63.

Asharani, P.V., Lianwu, Y., Gong, Z. \& Valiyaveettil, S., 2011. Comparison of the toxicity of silver, gold and platinum nanoparticles in developing zebrafish embryos. Nanotoxicology, 5, 43-54.

Asharani, P.V., Low Kah Mun, G., Hande, M.P. \& Valiyaveettil, S., 2009. Cytotoxicity and genotoxicity of silver nanoparticles in human cells. ACS Nano, 3, 279-90.

Bastus, N.G., Merkoci, F., Piella, J. \& Puntes, V., 2014. Synthesis of Highly Monodisperse Citrate-Stabilized Silver Nanoparticles of up to $200 \mathrm{~nm}$ : Kinetic Control and Catalytic Properties. Chemistry of Materials, 26, 28362846.

Benn, T., Cavanagh, B., Hristovski, K., Posner, J.D. \& Westerhoff, P., 2010. The Release of Nanosilver from Consumer Products Used in the Home. Journal of Environmental Quality, 39, 1875-1882.

Braakhuis, H.M., Kloet, S.K., Kezic, S., Kuper, F., Park, M.V., Bellmann, S., Van Der Zande, M., Le Gac, S., Krystek, P., Peters, R.J., Rietjens, I.M. \& Bouwmeester, H., 2015. Progress and future of in vitro models to study translocation of nanoparticles. Arch Toxicol, 89, 1469-95.

Carlson, C., Hussain, S.M., Schrand, A.M., Braydich-Stolle, L.K., Hess, K.L., Jones, R.L. \& Schlager, J.J., 2008. Unique cellular interaction of silver nanoparticles: size-dependent generation of reactive oxygen species. J Phys Chem B, 112, 13608-19.

Chanana, M. \& Liz-Marzán Luis, M., 2012. Coating matters: the influence of coating materials on the optical properties of gold nanoparticles. Nanophotonics. 199.

Connolly, M., Fernandez-Cruz, M.L., Quesada-Garcia, A., Alte, L., Segner, H. \& Navas, J.M., 2015. Comparative Cytotoxicity Study of Silver Nanoparticles (Ag NPs) in a Variety of Rainbow Trout Cell Lines (RTL-W1, RTH-149, RTG-2) and Primary Hepatocytes. Int J Environ Res Public Health, 12, 5386-405.

Cornelis, G., Hund-Rinke, K., Kuhlbusch, T., Van Den Brink, N. \& Nickel, C., 2014. Fate and Bioavailability of Engineered Nanoparticles in Soils: A Review. Critical Reviews in Environmental Science and Technology, 44, 2720-2764

De Lima, R., Seabra, A.B. \& Duran, N., 2012. Silver nanoparticles: a brief review of cytotoxicity and genotoxicity of chemically and biogenically synthesized nanoparticles. J Appl Toxicol, 32, 867-79.

Deng, X., Wang, Y., Zhang, F., Yin, Z., Hu, Q., Xiao, X., Zhou, Z., Wu, Y., Sheng, W. \& Zeng, Y., 2016. Acidic pH-induced charge-reversal nanoparticles for accelerated endosomal escape and enhanced microRNA modulation in cancer cells. Chem Commun (Camb), 52, 3243-6.

Eguchi, Y., Shimizu, S. \& Tsujimoto, Y., 1997. Intracellular ATP levels determine cell death fate by apoptosis or necrosis. Cancer Res, 57, 1835-40.

Evanoff, D.D., Jr. \& Chumanov, G., 2005. Synthesis and optical properties of silver nanoparticles and arrays. Chemphyschem, 6, 1221-31.

Foldbjerg, R., Dang, D.A. \& Autrup, H., 2011. Cytotoxicity and genotoxicity of silver nanoparticles in the human lung cancer cell line, A549. Arch Toxicol, 85, 743-50.

Foldbjerg, R., Irving, E.S., Hayashi, Y., Sutherland, D.S., Thorsen, K., Autrup, H. \& Beer, C., 2012. Global gene expression profiling of human lung epithelial cells after exposure to nanosilver. Toxicol Sci, 130, 145-57.

Foldbjerg, R., Jiang, X., Miclăuş, T., Chen, C., Autrup, H. \& Beer, C., 2015. Silver nanoparticles - wolves in sheep’s clothing? Toxicol. Res., 4, 563-575. 
Franci, G., Falanga, A., Galdiero, S., Palomba, L., Rai, M., Morelli, G. \& Galdiero, M., 2015. Silver nanoparticles as potential antibacterial agents. Molecules, 20, 8856-74.

Geranio, L., Heuberger, M. \& Nowack, B., 2009. The behavior of silver nanotextiles during washing. Environ Sci Technol, 43, 8113-8.

Gnanadhas, D.P., Ben Thomas, M., Thomas, R., Raichur, A.M. \& Chakravortty, D., 2013. Interaction of silver nanoparticles with serum proteins affects their antimicrobial activity in vivo. Antimicrob Agents Chemother, 57, 4945-55.

Gwinn, M.R. \& Vallyathan, V., 2006. Nanoparticles: health effects--pros and cons. Environ Health Perspect, 114, 1818-25.

Hagendorfer, H., Kaegi, R., Parlinska, M., Sinnet, B., Ludwig, C. \& Ulrich, A., 2012. Characterization of silver nanoparticle products using asymmetric flow field flow fractionation with a multidetector approach--a comparison to transmission electron microscopy and batch dynamic light scattering. Anal Chem, 84, 2678-85.

Haider, A. \& Kang, I.K., 2015. Preparation of Silver Nanoparticles and Their Industrial and Biomedical Applications: A Comprehensive Review. Advances in Materials Science and Engineering, 2015, 16.

Hwang, I.S., Hwang, J.H., Choi, H., Kim, K.J. \& Lee, D.G., 2012. Synergistic effects between silver nanoparticles and antibiotics and the mechanisms involved. J Med Microbiol, 61, 1719-26.

Jeong, Y.S., Oh, W.K., Kim, S. \& Jang, J., 2011. Cellular uptake, cytotoxicity, and ROS generation with silica/conducting polymer core/shell nanospheres. Biomaterials, 32, 7217-25.

Johnston, H.J., Hutchison, G., Christensen, F.M., Peters, S., Hankin, S. \& Stone, V., 2010. A review of the in vivo and in vitro toxicity of silver and gold particulates: particle attributes and biological mechanisms responsible for the observed toxicity. Crit Rev Toxicol, 40, 328-46.

Kennell, D., 2009. Nanotechnology: An Industrial Revolution? Monthly Review MRZine.

Kloet, S.K., Walczak, A.P., Louisse, J., Van Den Berg, H.H., Bouwmeester, H., Tromp, P., Fokkink, R.G. \& Rietjens, I.M., 2015. Translocation of positively and negatively charged polystyrene nanoparticles in an in vitro placental model. Toxicol In Vitro, 29, 1701-10.

Kreuter, J., 2004. Influence of the surface properties on nanoparticle-mediated transport of drugs to the brain. J Nanosci Nanotechnol, 4, 484-8.

Landsiedel, R., Fabian, E., Ma-Hock, L., Van Ravenzwaay, B., Wohlleben, W., Wiench, K. \& Oesch, F., 2012. Toxico-/ biokinetics of nanomaterials. Arch Toxicol, 86, 1021-60.

Lara, H.H., Ayala-Nunez, N.V., Ixtepan-Turrent, L. \& Rodriguez-Padilla, C., 2010. Mode of antiviral action of silver nanoparticles against HIV-1. J Nanobiotechnology, 8, 1.

Lemasters, J.J., Nieminen, A.L., Qian, T., Trost, L.C., Elmore, S.P., Nishimura, Y., Crowe, R.A., Cascio, W.E., Bradham, C.A., Brenner, D.A. \& Herman, B., 1998. The mitochondrial permeability transition in cell death: a common mechanism in necrosis, apoptosis and autophagy. Biochim Biophys Acta, 1366, 177-96.

Liu, W., Wu, Y., Wang, C., Li, H.C., Wang, T., Liao, C.Y., Cui, L., Zhou, Q.F., Yan, B. \& Jiang, G.B., 2010. Impact of silver nanoparticles on human cells: effect of particle size. Nanotoxicology, 4, 319-30.

Makama, S., Peters, R., Undas, A. \& Van Den Brink, N.W., 2015. A novel method for the quantification, characterisation and speciation of silver nanoparticles in earthworms exposed in soil. Environmental Chemistry, 12, 643-651.

Nadesh, R., Narayanan, D., P, R.S., Vadakumpully, S., Mony, U., Koyakkutty, M., Nair, S.V. \& Menon, D., 2013. Hematotoxicological analysis of surface-modified and -unmodified chitosan nanoparticles. J Biomed Mater Res A, 101, 2957-66.

Nafee, N., Schneider, M., Schaefer, U.F. \& Lehr, C.M., 2009. Relevance of the colloidal stability of chitosan/PLGA nanoparticles on their cytotoxicity profile. Int J Pharm, 381, 130-9.

Park, M.V., Neigh, A.M., Vermeulen, J.P., De La Fonteyne, L.J., Verharen, H.W., Briede, J.J., Van Loveren, H. \& De Jong, W.H., 2011. The effect of particle size on the cytotoxicity, inflammation, developmental toxicity and genotoxicity of silver nanoparticles. Biomaterials, 32, 9810-7.

Pavlin, M. \& Bregar, V., 2012. Stability of nanoparticle suspension in different biologically relevant media. Digest Journal of Nanomaterials and Biostructures, 7, 1389-1400.

Peterson, C., 2000. Taking technology to the molecular level. Computer, 33, 46-+.

Powers, C.M., Badireddy, A.R., Ryde, I.T., Seidler, F.J. \& Slotkin, T.A., 2011a. Silver Nanoparticles Compromise Neurodevelopment in PC12 Cells: Critical Contributions of Silver Ion, Particle Size, Coating, and Composition. Environmental Health Perspectives, 119, 37-44. 
Powers, C.M., Badireddy, A.R., Ryde, I.T., Seidler, F.J. \& Slotkin, T.A., 2011b. Silver nanoparticles compromise neurodevelopment in PC12 cells: critical contributions of silver ion, particle size, coating, and composition. Environ Health Perspect, 119, 37-44.

Prabhu, S. \& Poulose, E.K., 2012. Silver nanoparticles: mechanism of antimicrobial action, synthesis, medical applications, and toxicity effects. International Nano Letters, 2, 32.

Reidy, B., Haase, A., Luch, A., Dawson, K.A. \& Lynch, I., 2013. Mechanisms of Silver Nanoparticle Release, Transformation and Toxicity: A Critical Review of Current Knowledge and Recommendations for Future Studies and Applications. Materials, 6, 2295-2350.

Roohani-Esfahani, S.I., Nouri-Khorasani, S., Lu, Z., Appleyard, R. \& Zreiqat, H., 2010. The influence hydroxyapatite nanoparticle shape and size on the properties of biphasic calcium phosphate scaffolds coated with hydroxyapatite-PCL composites. Biomaterials, 31, 5498-509.

Samberg, M.E., Orndorff, P.E. \& Monteiro-Riviere, N.A., 2011. Antibacterial efficacy of silver nanoparticles of different sizes, surface conditions and synthesis methods. Nanotoxicology, 5, 244-53.

Shang, L., Nienhaus, K., Jiang, X., Yang, L., Landfester, K., Mailander, V., Simmet, T. \& Nienhaus, G.U., $2014 a$. Nanoparticle interactions with live cells: Quantitative fluorescence microscopy of nanoparticle size effects. Beilstein J Nanotechnol, 5, 2388-97.

Shang, L., Nienhaus, K. \& Nienhaus, G.U., 2014b. Engineered nanoparticles interacting with cells: size matters. J Nanobiotechnology, 12, 5.

Shannahan, J.H., Lai, X., Ke, P.C., Podila, R., Brown, J.M. \& Witzmann, F.A., 2013. Silver nanoparticle protein corona composition in cell culture media. PLoS One, 8, e74001.

Shenashen, M.A., El-Safty, S.A. \& Elshehy, E.A., 2014. Synthesis, Morphological Control, and Properties of Silver Nanoparticles in Potential Applications. Particle \& Particle Systems Characterization, 31, 293-316.

Shoults-Wilson, W.A., Reinsch, B.C., Tsyusko, O.V., Bertsch, P.M., Lowry, G.V. \& Unrine, J.M., 2011. Effect of silver nanoparticle surface coating on bioaccumulation and reproductive toxicity in earthworms (Eisenia fetida). Nanotoxicology, 5, 432-44.

Silver, S. \& Phung, L.T., 1996. Bacterial heavy metal resistance: new surprises. Annu Rev Microbiol, 50, 753-89.

Sondi, I. \& Salopek-Sondi, B., 2004. Silver nanoparticles as antimicrobial agent: a case study on E. coli as a model for Gram-negative bacteria. J Colloid Interface Sci, 275, 177-82.

Suresh, A.K., Pelletier, D.A., Wang, W., Morrell-Falvey, J.L., Gu, B. \& Doktycz, M.J., 2012. Cytotoxicity induced by engineered silver nanocrystallites is dependent on surface coatings and cell types. Langmuir, 28, 2727-35.

Van Der Ploeg, M.J., Handy, R.D., Waalewijn-Kool, P.L., Van Den Berg, J.H., Herrera Rivera, Z.E., Bovenschen, J., Molleman, B., Baveco, J.M., Tromp, P., Peters, R.J., Koopmans, G.F., Rietjens, I.M. \& Van Den Brink, N.W., 2014a. Effects of silver nanoparticles (NM-300K) on Lumbricus rubellus earthworms and particle characterization in relevant test matrices including soil. Environ Toxicol Chem, 33, 743-52.

Van Der Ploeg, M.J., Van Den Berg, J.H., Bhattacharjee, S., De Haan, L.H., Ershov, D.S., Fokkink, R.G., Zuilhof, H., Rietjens, I.M. \& Van Den Brink, N.W., 2014b. In vitro nanoparticle toxicity to rat alveolar cells and coelomocytes from the earthworm Lumbricus rubellus. Nanotoxicology, 8, 28-37.

Vance, M.E., Kuiken, T., Vejerano, E.P., Mcginnis, S.P., Hochella, M.F., Jr., Rejeski, D. \& Hull, M.S., 2015. Nanotechnology in the real world: Redeveloping the nanomaterial consumer products inventory. Beilstein J Nanotechnol, 6, 1769-80.

Venter, G., Oerlemans, F.T.J.J., Wijers, M., Willemse, M., Fransen, J.a.M. \& Wieringa, B., 2014. Glucose Controls Morphodynamics of LPS-Stimulated Macrophages. PLoS ONE, 9, e96786.

Vonna, L., Wiedemann, A., Aepfelbacher, M. \& Sackmann, E., 2007. Micromechanics of filopodia mediated capture of pathogens by macrophages. Eur Biophys J, 36, 145-51.

Wijnhoven, S.W.P., Oomen, A.G., Sips, A.J.a.M., Bourgeois, F.C., Te Dorsthorst, G.J.P.M., Kooi, M.W. \& Bakker, M.I., 2010. Development of an inventory for consumer products containing nanomaterials Final Report RIVM Report 000201006, 103.

Wijnhoven, S.W.P., Peijnenburg, W.J.G.M., Herberts, C.A., Hagens, W.I., Oomen, A.G., Heugens, E.H.W., Roszek, B., Bisschops, J., Gosens, I., Van De Meent, D., Dekkers, S., De Jong, W.H., Van Zijverden, M., Sips, A.J.a.M. \& Geertsma, R.E., 2009. Nano-silver - a review of available data and knowledge gaps in human and environmental risk assessment. Nanotoxicology, 3, 109-U78. 
Yang, X., Gondikas, A.P., Marinakos, S.M., Auffan, M., Liu, J., Hsu-Kim, H. \& Meyer, J.N., 2012. Mechanism of silver nanoparticle toxicity is dependent on dissolved silver and surface coating in Caenorhabditis elegans. Environ Sci Technol, 46, 1119-27.

Yen, H.J., Hsu, S.H. \& Tsai, C.L., 2009. Cytotoxicity and immunological response of gold and silver nanoparticles of different sizes. Small, 5, 1553-61.

Yu, S.J., Yin, Y.G. \& Liu, J.F., 2013. Silver nanoparticles in the environment. Environ Sci Process Impacts, 15, 78-92.

Zhao, L.M., Shi, L.E., Zhang, Z.L., Chen, J.M., Shi, D.D., Yang, J. \& Tang, Z.X., 2011. Preparation and Application of Chitosan Nanoparticles and Nanofibers. Brazilian Journal of Chemical Engineering, 28, 353-362. 


\section{SUPPLEMENTARY MATERIALS CHAPTER 6}

\section{MATERIALS AND METHODS}

\section{Synthesis of Ag NPs}

MilliQ water $(18.2 \mathrm{M} \Omega / \mathrm{cm}$ ) was used for all Ag NPs syntheses. Colloidal, dispersed Ag NPs of two different sizes (20 and $50 \mathrm{~nm}$ ) were prepared separately, following a kinetically controlled seeded-growth method previously reported (Bastus et al. 2014) with slight modifications. The approach is based on the reduction of silver nitrate $\left(\mathrm{AgNO}_{3}\right)$ in the presence of two competing reducing agents, tannic acid (TA) and trisodium citrate hexahydrate (SC) at $100^{\circ} \mathrm{C}$. In order to produce large amounts (grams) of Ag NPs in the present study, a $10 \mathrm{~L}$ batch reactor was used and the concentration of the $\mathrm{AgNO}_{3}$ ( $\mathrm{Ag} \mathrm{NP}$ precursor) was increased by a factor of 10, while all other parameters remained the same with that reported previously (Bastus et al. 2014).

First, early nucleated Ag NP seeds were formed by injecting $5 \mathrm{~mL}$ of $2 \mathrm{M} \mathrm{AgNO}_{3}$ into a boiling solution containing SC $(5 \mathrm{mM})$ and TA $(0.025-0.075 \mathrm{mM})$, under continuous vigorous stirring. These seeds were further grown to the desired sizes by injecting more of the silver precursor dropwise to avoid formation of new seeds. During the growth phase of the seeds, the temperature of the solution was reduced to $90^{\circ} \mathrm{C}$, while slowly adding up to $15 \mathrm{~mL}$ of the $2 \mathrm{M} \mathrm{AgNO}_{3}$ into the reactor. Under these conditions, 10-20 L of a stable, highly monodisperse and colloidal Ag NP solutions (0.25-0.5 g/L or 2.3 - 4.6 mM) were produced for each size.

The solutions containing the synthesized Ag NPs were concentrated to about $6 \%$ of their original volume by ultrafiltration using a tangential flow fractionation (TFF) system (KrosFlo ${ }^{\circ}$ Research II TFF Systems, Spectrum Laboratories Inc.). Negative, positive, and neutral Ag NPs were obtained by subsequently mixing about $200 \mathrm{~mL}$ each of the concentrated solutions of Ag NPs with either $0.01 \mathrm{mM}$ bovine serum albumin (BSA), $0.08 \mathrm{mM}$ chitosan (Chit) or $0.05 \mathrm{mM}$ polyvinylpyrrolidone (PVP) to generate negative Ag NP_BSA, positive Ag NP_Chit and neutral Ag NP_PVP, respectively. To prevent corrosion and facilitate easy transport, the Ag NPs were lyophilized, layered over with argon and kept under dark conditions until use. Thus, six different Ag NPs were synthesized consisting of three types based on surface coating/charge, each at two different sizes.

An aqueous suspension of a negatively charged $50 \mathrm{~nm}$ PVP-coated Ag NP (Ag NP_NC) was obtained from NanoComposix (San Diego, US) and included in this study for comparison with the synthesized Ag NPs. Based on the manufacturer's information, the Ag NP_NC is reported to have mean core- and hydrodynamic diameters of $54.8 \pm 10 \mathrm{~nm}$ and $72 \pm 14.4 \mathrm{~nm}$ respectively. The stock suspension of the pristine Ag NP has a mass concentration of $5.0 \mathrm{mg}$ 
$\mathrm{Ag} / \mathrm{mL}$, and a particle number concentration of $5.2 \times 10^{12}$ particles $/ \mathrm{mL}$. The surface area was $9.8 \mathrm{~m}^{2} / \mathrm{g}$ while surface charge (zeta-potential) was $-37.8 \mathrm{mV}$. The DLS and zeta potential in both milliQ and DMEM were confirmed in our laboratory, and details have been presented earlier (Makama et al. 2015).

\section{Ag NP Characterization}

Prior to exposure experiments, pristine Ag NPs were dispersed in Dulbecco's modified Eagle's medium, DMEM (Invitrogen Breda, The Netherlands), as well as milliQ water and characterized by transmission electron microscopy (TEM), UV-Vis spectroscopy, dynamic light scattering (DLS), and zeta-potential measurements ( $\zeta$-potential). A combination of different techniques was used to characterize the Ag NPs and to monitor proper coating of NPs surfaces and their stability in the different media.

TEM images were acquired with a FEI Magellane 400L SEM electron microscope operating at scanning TEM (STEM) mode and low accelerating voltage $(20 \mathrm{kV})$ and a JEOL 1010 electron microscope operating at an accelerating voltage of $80 \mathrm{kV}$. Samples for TEM were prepared by drop casting onto carbon-coated TEM grids. The grids were left to dry at room temperature. Images were acquired in different parts of the grid at different magnifications. More than 250 particles were computer-analyzed and measured to calculate the size distribution of each type of Ag NPs.

UV-Vis spectroscopy is a very common and well-known analytical technique (Evanoff and Chumanov 2005; Pan 2015), and a Shimadzu UV-2400 spectrophotometer was used to measure the localized surface Plasmon resonance (SPR) peak. Here, $1 \mathrm{~mL}$ of the Ag NPs suspension was placed in a cuvette and the spectrum (UV-Vis) acquired in the $300-800 \mathrm{~nm}$ range. Several metallic NPs, such as gold and silver, exhibit a characteristic absorbance maximum in the visible range (SPR peak). This characteristic wavelength is highly sensitive to the ENP size, surface modifications and aggregation and thus a useful tool to characterize the state of the NPs in solution.

Hydrodynamic sizes of the Ag NPs were estimated by DLS, while particle surface charges ( $\zeta$-potentials) were measured using Malvern Zetasizer Nano ZS (Malvern Instruments, UK). A light source wavelength of $532 \mathrm{~nm}$ and a fixed scattering angle of $173^{\circ}$ were set for DLS measurement. Ag NPs suspensions $(1 \mathrm{~mL})$ were dispensed in a cuvette and the instrument was set with the specific parameters of refractive index and absorption coefficient of the material and the viscosity of the solvent. All measurements were conducted at least three times. Ag NPs characterization was done in both milliQ water and DMEM since the exposure was carried out in the cell culture medium. 


\section{Effect assessment:}

\section{MTT Assay}

At the end of the $24 \mathrm{hr}$ exposure, $10 \mu \mathrm{L}$ of the tetrazolium salt 3-(4,5-dimethylthiazol-2-yl)-2,5-diphenyltetrazolium bromide (MTT) solution at $5 \mathrm{mg} / \mathrm{mL}$ in PBS was added to each well and incubated under the same conditions as described above for $90 \mathrm{~min}$. The exposure medium containing MTT was then removed and $100 \mu \mathrm{L}$ pure dimethyl sulfoxide, DMSO (Acros Organics, Geel, BE) was added to solubilize the crystals of formazan salts. This was followed by shaking the plates in the dark for $10 \mathrm{~min}$. The dissolved formazan produces a purple color and its absorbance was measured at $562 \mathrm{~nm}$ spectrophotometrically (Kloet et al. 2015).

\section{Adenosine Triphosphate (ATP) Assay}

A bioluminescent somatic cell assay kit (Sigma-Aldrich', St Louis, MO, US), was used to determine the ATP content of mouse macrophage cells after exposure. In this assay, ATP is the limiting reagent and the light emitted and measured is proportional to the amount of ATP released from the cells, which is in turn determined by the number of viable cells present. Exposure to Ag NPs for this assay was conducted in white 96-well plates (Packard White plates, Perkin-Elmer), using DMEM without phenol red, but with penicillin/streptomycin (Gibco $)$ $1 \%(\mathrm{v} / \mathrm{v})$. After exposure, cell culture media was removed and replaced with $25 \mu \mathrm{L}$ each of fresh medium and somatic cell ATP releasing agent from the kit. The plates were placed on a shaker for 10 mins in the dark, then transferred to the luminometer where the ATP assay working mix (containing luciferin, luciferase and $\mathrm{MgSO}_{4}$ ) was added automatically to each well before measuring the luminescence.

\section{Mitochondrial permeability Transition-Pore (MPTP) Assay}

A commercial kit from Molecular Probes (Leiden, NL), MitoProbe ${ }^{\mathrm{TM}}$ Transition Pore (MPTP) Assay kit (M34153) was used for this assay. After exposure, the culture medium in all wells was replaced by Hank's balanced salt solution, HBSS (Invitrogen, Breda NL), containing calcium but without serum, phenol red or $\mathrm{NaHCO}_{3}$. Cells in all except the positive control wells were loaded with a mixture of cobalt chloride $\left(\mathrm{CoCl}_{2}\right)$ and an acetoxymethyl ester form of a calcein dye (Calcein AM) at $1 \mathrm{mM}$ and $5 \mu \mathrm{M}$ respectively, followed by a $15 \mathrm{~min}$ incubation. Calcein AM passively diffuses into cells and accumulates in the mitochondria, where it is de-esterified by intracellular esterases to liberate the highly fluorescent calcein. Subsequently, calcein does not cross the mitochondrial or plasma membrane in appreciable amounts over short periods of time, making it conducive for selective marking of the mitochondria (Martel et al. 2012). This selectivity is achieved by adding $\mathrm{CoCl}_{2}$ which quenches the cytosolic fluorescence, while mitochondrial fluorescence is maintained in intact mitochondria since $\mathrm{CoCl}_{2}$ is incapable of entering this organelle. 
The cells in positive control wells were treated during this loading step with an ionophore (ionomycin) at $10 \mu \mathrm{M}$, which was dissolved in the Calcein $\mathrm{AM} / \mathrm{CoCl}_{2}$ mixture above. Ionomycin allows the entry of $\mathrm{Ca}^{2+}$ into the mitochondria in excess, triggering the opening of the mitochondrial permeability transition pore (MPTP). This results in the loss of calcein fluorescence into the cytosolic compartment where it is quenched. Fluorescence was measured at $\lambda 485 / 530 \mathrm{~nm}$. The relative intensity of fluorescence compared to the blank control (100\%) is indicative of the integrity of mitochondrial permeability transition pore after exposure to Ag NPs.

\section{Intracellular Reactive Oxygen Species (ROS) generation; DCF-Assay}

The generation of intracellular ROS was determined using a slightly modified method earlier described (Hussain et al. 2006). Cells were preloaded with $20 \mu \mathrm{M}$ 2',7'-dichlorodihydrofluorescein diacetate $\left(\mathrm{H}_{2} \mathrm{DCF}-\mathrm{DA}\right)$ dye from Molecular probes (Carlsbad, CA, US) for $45 \mathrm{~min}$. After this, the medium containing the dye was removed and the cells were exposed to the various concentrations of Ag NPs for $6 \mathrm{~h}$. Fluorescence was measured at $\lambda 485 / 520 \mathrm{~nm}$ at the end of the exposure and expressed as percentages relative to the blank control (optimal healthy cell ROS value set at $100 \%)$.

\section{TNF- $\alpha$ induction}

At the end of the $24 \mathrm{~h}$ exposure period, $80 \mu \mathrm{L}$ supernatants containing cytokines were collected from each well and stored at $-80{ }^{\circ} \mathrm{C}$ until analyzed for secreted levels of TNF- $\alpha$ by a solid phase sandwich Enzyme-Linked Immuno-Sorbent Assay (ELISA) using a Mouse TNF- $\alpha$ ELISA kit (Invitrogen ${ }^{\mathrm{TM}}$, Paisley, UK). Analysis was conducted according to the manufacturer's instruction, running calibration and other standards for every 12 × 8 strip-well plates. Briefly, samples were added onto microtiter strip wells pre-coated with specific monoclonal TNF- $\alpha$ antibodies. Next, a polyclonal biotinylated conjugate was added as a second antibody and the plates were incubated at room temperature for $90 \mathrm{~min}$. During incubation, any secreted TNF- $\alpha$ in the samples will form a sandwich with these antibodies. After a washing step to get rid of excess antibodies, streptavidin-peroxidase was added to complete the four-member sandwich by binding to the biotinylated antibody during another $30 \mathrm{~min}$ incubation. A wash step gets rid of all unbound enzymes before a substrate solution is added to be acted upon by the bound enzymes to produce a color whose optical density at $450 \mathrm{~nm}$ is directly proportional to the concentration of TNF- $\alpha$ in the sample. 


\section{Controls of Assays}

\section{Effect assessment controls}
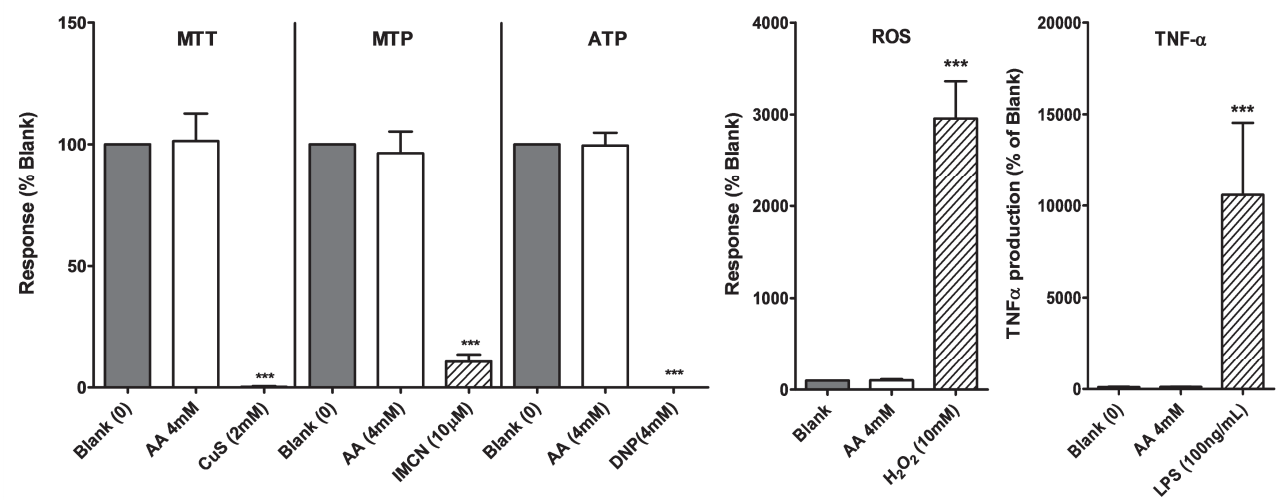

Figure S1. Controls for effect assessment experiments - MTT (cell viability), MPTP opening, ATP production, ROS generation, and TNF- $\alpha$ induction assays. Results are expressed as percentages (mean \pm standard deviation, $\mathrm{n}=3$ ) relative to the blank, set at $100 \%$. Statistical significance compared to control is indicated by ${ }^{* *}(\mathrm{p} \leq 0.001)$. Positive controls showed clear effects, while exposure to acetic acid (AA) at $4 \mathrm{mM}$ was similar to the blank control. DNP, dinitrophenol; IMCN, ionomycin; LPS, lipopolysaccharide.

\section{Ag NPs Characterization}

Table S1. Ag NP characterization showing the hydrodynamic sizes of Ag NPs measured by dynamic light scattering (DLS), surface Plasmon Resonance (SPR) peaks and $\zeta$-potentials. Measurements were performed before and after surface coating of NPs

\begin{tabular}{|c|c|c|c|c|c|c|c|}
\hline & & \multicolumn{2}{|c|}{ BSA (negative) } & \multicolumn{2}{|c|}{ Chitosan (positive) } & \multicolumn{2}{|c|}{ PVP (neutral) } \\
\hline & & $20 \mathrm{~nm}$ & $50 \mathrm{~nm}$ & $20 \mathrm{~nm}$ & $50 \mathrm{~nm}$ & $20 \mathrm{~nm}$ & $50 \mathrm{~nm}$ \\
\hline TEM Size & $\mathrm{nm}$ & $19.5 \pm 5.4$ & $51.1 \pm 5.7$ & $18.2 \pm 5.1$ & $51.9 \pm 6.4$ & $24.0 \pm 4.6$ & $51.0 \pm 6.1$ \\
\hline Size $^{\mathrm{a}}$ & $\mathrm{nm}$ & $41.5 \pm 1.3$ & $65.3 \pm 0.1$ & $247.8 \pm 2.1$ & $241.8 \pm 5.1$ & $46.8 \pm 0.1$ & $68.4 \pm 0.6$ \\
\hline Size $^{\mathrm{b}}$ & $\mathrm{nm}$ & $69.9 \pm 3.2$ & $86.3 \pm 1.0$ & $209.1 \pm 1.4$ & $234.5 \pm 1.2$ & $63.0 \pm 0.2$ & $71.3 \pm 2.0$ \\
\hline$\zeta$-potential ${ }^{\mathrm{a}}$ & $\mathrm{meV}$ & $-29.0 \pm 2.0$ & $-37.0 \pm 2.0$ & $+8.0 \pm 2.0$ & $+7.0 \pm 1.0$ & $-16 \pm 2.0$ & $-25.0 \pm 2.0$ \\
\hline$\zeta$-potential ${ }^{\mathrm{b}}$ & $\mathrm{meV}$ & $-25.5 \pm 2.3$ & $-23.0 \pm 2.0$ & $-16.4 \pm 2.3$ & $-21.3 \pm 1.4$ & $-16.2 \pm 2.3$ & $-19.3 \pm 0.7$ \\
\hline UV-vis SPR peaks ${ }^{c}$ & $\mathrm{~nm}$ & 405 & 441 & 405 & 441 & 405 & 441 \\
\hline UV-vis SPR peaks ${ }^{\mathrm{a}}$ & $\mathrm{nm}$ & 413 & 448 & 413 & 449 & 408 & 443 \\
\hline UV-vis SPR peaks ${ }^{b}$ & $\mathrm{Nm}$ & 412 & 447 & 414 & 450 & 408 & 444 \\
\hline
\end{tabular}

meV, milli-electron volts; c, before coating; b and c, after coating in $\mathrm{H}_{2} \mathrm{O}$ or DMEM respectively. 


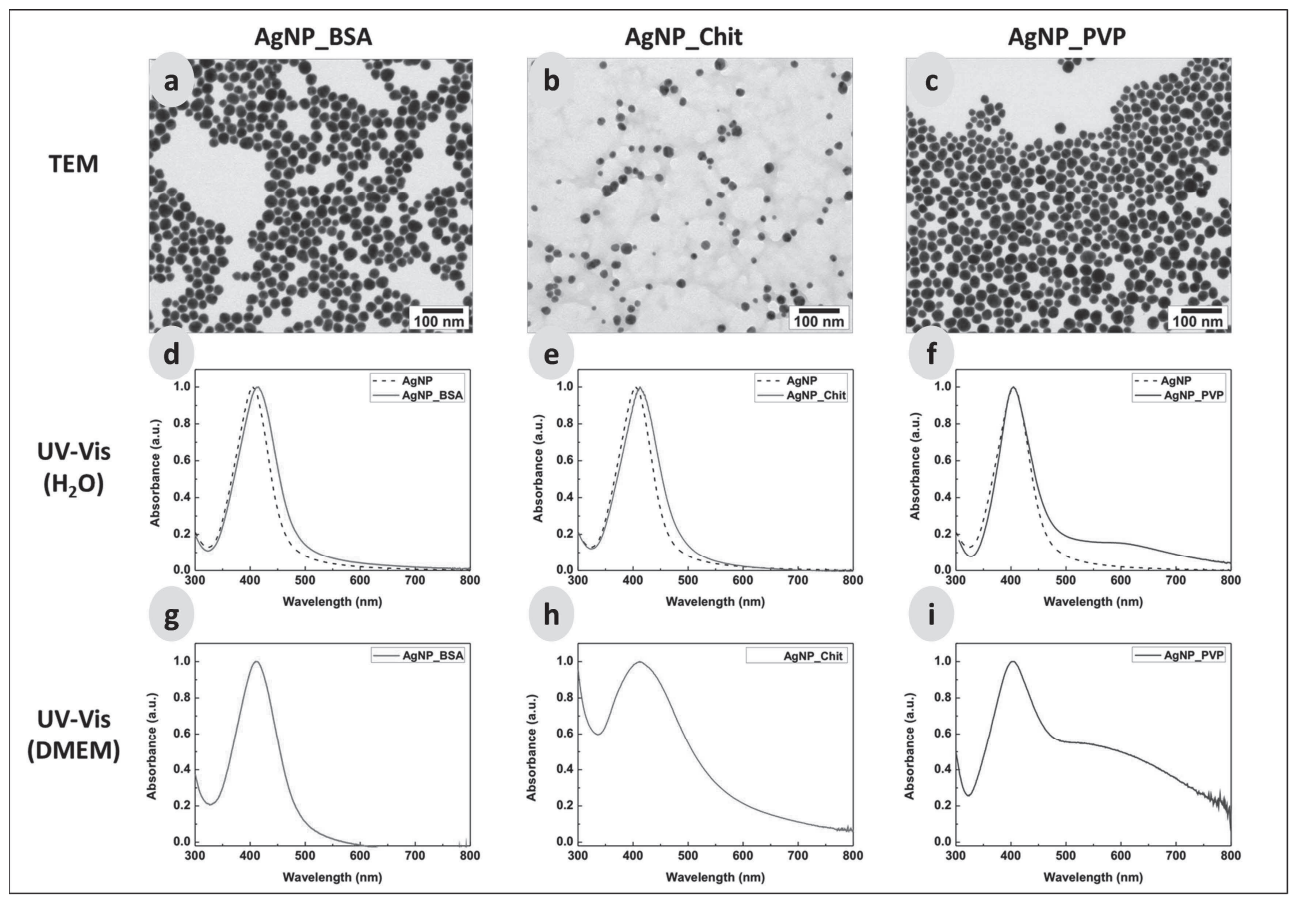

Figure S2. Ag NPs characterization results for 20nm size group. TEM images (a - c) of re-suspended Ag NPs showing quasi-spherical particles within the expected size ranges, with overall average diameter of $20.6 \pm 5.0 \mathrm{~nm}$. UV-Vis also shows SPR peaks within the expected wavelength range $300-600 \mathrm{~nm}$ both in milliQ water and in cell culture medium ( $\mathrm{d}-\mathrm{f}$ and $\mathrm{g}-\mathrm{i}$, respectively). The UV-Vis SPR curves of coated and non-coated Ag NPs nicely overlaps (d - f), with the SPR curves of the coated NPs exhibiting a red-shift indicative of the effect of Ag NPs coating. When Ag NP_Chit and Ag NP_PVP were diluted in Dulbecco's modified Eagle's medium (DMEM), some agglomerations appear to set in as indicated by the changes in the SPR curves (Fig. 2e-f compared with $2 \mathrm{~h}-\mathrm{i}$ ).

\section{Confocal localization and uptake dynamics of Ag NPs}

RAW 264.7 cells were seeded in a glass bottom 8-well $\mu$-Slides (Ibidi GmbH, Martinsried, $\mathrm{DE})$ at a density of $5 \times 10^{5}$ cells/mL growth medium, and incubated at $37^{\circ} \mathrm{C}$ in humidified air (plus $5 \% \mathrm{CO}_{2}$ ) to attain $\sim 80 \%$ adherent confluency. Ag NPs exposures were performed only with the $50 \mathrm{~nm}$ Ag NP_BSA and Ag NP_PVP at $0.1-5 \mu \mathrm{g} / \mathrm{mL}$, and characterized after 2.5 and $24 \mathrm{~h}$ of exposure. The exposure was carried out immediately before microscopic measurements.

Imaging was done on a Confocal Laser Scanning LSM 510-META on an Axiovert 200M microscope (Carl Zeiss, Jena, DE) equipped with a 40x/1.3 Oil DIC Plan Neofluar or 63x/1.4 Oil DIC Plan Apochromat objective. Localizations were obtained with optical $x y$-planes of 1.0 
um depth (z) in adhered cells with $40 \%$ of a $1 \mathrm{~mW} 543 \mathrm{~nm}$ diode laser excitation using LP560 and standardized zoom and exposures (scan speed 6) and gain settings (839) for all acquisitions. By screening the fluorescence spectra of Ag NPs after excitation by a $488 \mathrm{~nm}, 514 \mathrm{~nm}$ or $543 \mathrm{~nm}$ laser with a multi array of detectors in the META channel, it appeared that the $543 \mathrm{~nm}$ absorption and strong LP560 fluorescence provided the most sensitive and specific configuration to be used for imaging. Controls and exposed cells were imaged in the same way, with no background signal in the controls. Uptake dynamics were obtained by image acquisitions in time series over 2.5 and $24 \mathrm{~h}$ periods. Images were similarly processed with Zeiss ZEN software and ImageJ v.1.49o (Rasband, W.S., ImageJ, U. S. National Institutes of Health, Bethesda, Maryland, US, http://imagej.nih.gov/ij/, 1997-2015).

In this study, Ag NPs taken up by macrophages could be excited with $40 \%$ of a $1 \mathrm{~mW} 543 \mathrm{~nm}$ laser and detected with a long pass (LP) $560 \mathrm{~nm}$ filter. Using a multi-detector array (i.e. the META detector in lambda channel mode of a Zeiss LSM510-M), emission wavelengths that could induce the most bright fluorescence were analyzed. Base on this, the most sensitive channel mode imaging configuration, without any auto fluorescent signal from the cells was chosen. To obtain sensitive and specific detection of Ag NPs, several laser/filter configurations were tested, and signals obtained were most bright at $543 \mathrm{~nm}$. A multi-detector array (560-750 nm) showed that emission photons induced after $543 \mathrm{~nm}$ excitation, originated from $560-600 \mathrm{~nm}$ emissions ( $~ 80 \%$ of output) decreasing towards higher wavelengths. Since macrophage intrinsic fluorescence was not detected with these settings, we used a channel mode with $560 \mathrm{LP}$ detection to localize the Ag NPs. Due to the relatively slow point scanning speed, the Ag NPs were best detected during and after cellular uptake since reduced dynamics increases signal detection. A dose dependent signal increase was observed (Table S2, Fig. S3) with different Ag NPs tested, similar to earlier reports (Zucker et al. 2013).

Table S2.Uptake of different types of Ag NPs by RAW264.7 cells exposed for 2.5 and $24 \mathrm{~h}$ to different concentrations of Ag NPs

\begin{tabular}{lcccc}
\hline $\begin{array}{l}\text { Concentration } \boldsymbol{\mu g} / \mathrm{mL} \\
\text { Duration }\end{array}$ & \multicolumn{2}{c}{ Ag NP_BSA } & \multicolumn{2}{c}{ Ag NP_PVP } \\
\hline 0.1 & $\mathbf{2 . 5} \mathbf{h}$ & $\mathbf{2 4} \mathbf{~ h}$ & $\mathbf{2 . 5} \mathbf{~ h}$ & $\mathbf{2 4} \mathbf{~ h}$ \\
0.5 & $12.2 \pm 3.4$ & $10.6 \pm 1.6$ & $6.5 \pm 1.6$ & $6.3 \pm 1.1$ \\
1 & n.m. & n.m. & $7.7 \pm 1.1$ & $6.5 \pm 0.5$ \\
5 & $9.8 \pm 1.5$ & $10.9 \pm 1.3$ & $8.7 \pm 1.6$ & $6.3 \pm 1.3$ \\
\hline
\end{tabular}

n.m., not measured 
The fluorescence signals correlating to the Ag NPs were quantified based on mean pixel intensity of fields of view of representative cytoplasmic domains (Fig. S3 and S4), and were expressed as arbitrary units (AU). At identical exposures as well as imaging and processing conditions, these fluorescence intensity values reflect uptake of the different Ag NPs by macrophage cells. To allow Ag NP uptake comparisons, quantifications of cytoplasmic dense regions from randomly selected cells with similar cellular confluency $(70-80 \%)$ and $z$-depths were made (Fig. S3). The selection of representative cytoplasm dense regions was based on the visual images. This selection was then applied to the fluorescence signal, and the AU quantified. In this way, regions analyzed were selected based on the occurrence of cytoplasm, without the bias of occurrence of silver signal. Based on the AUs estimated, the uptake of the negatively charged Ag NP_BSA was rapid and most significant. Due to agglomeration in the suspension and growth medium, it was not possible to assess the uptake of Ag NP_Chit and will not be discussed further. The cellular uptake of Ag NP_BSA was twice that of Ag NP_ PVP at similar exposure concentrations and time (Table S2), although the uptake efficiency of individual cells does vary a lot. 


\section{REFERENCES}

Bastus NG, Merkoci F, Piella J, Puntes V (2014) Synthesis of Highly Monodisperse Citrate-Stabilized Silver Nanoparticles of up to $200 \mathrm{~nm}$ : Kinetic Control and Catalytic Properties Chem Mater 26:2836-2846 doi:10.1021/ $\mathrm{cm} 500316 \mathrm{k}$

Evanoff DD, Jr., Chumanov G (2005) Synthesis and optical properties of silver nanoparticles and arrays Chemphyschem 6:1221-1231 doi:10.1002/cphc.200500113

Hussain SM, Javorina AK, Schrand AM, Duhart HM, Ali SF, Schlager JJ (2006) The interaction of manganese nanoparticles with PC-12 cells induces dopamine depletion Toxicol Sci 92:456-463 doi:10.1093/toxsci/ kfl020

Kloet SK et al. (2015) Translocation of positively and negatively charged polystyrene nanoparticles in an in vitro placental model Toxicol In Vitro 29:1701-1710 doi:10.1016/j.tiv.2015.07.003

Makama S, Peters R, Undas A, van den Brink NW (2015) A novel method for the quantification, characterisation and speciation of silver nanoparticles in earthworms exposed in soil Environmental Chemistry 12:643-651 doi:10.1071/EN15006

Martel C, Huynh le H, Garnier A, Ventura-Clapier R, Brenner C (2012) Inhibition of the Mitochondrial Permeability Transition for Cytoprotection: Direct versus Indirect Mechanisms Biochem Res Int 2012:213403 doi:10.1155/2012/213403

Pan YL (2015) Detection and characterization of biological and other organic-carbon aerosol particles in atmosphere using fluorescence J Quant Spectrosc Ra 150:12-35 doi:10.1016/j.jqsrt.2014.06.007

Zucker RM, Daniel KM, Massaro EJ, Karafas SJ, Degn LL, Boyes WK (2013) Detection of silver nanoparticles in cells by flow cytometry using light scatter and far-red fluorescence Cytometry Part A 83:962-972 doi:10.1002/ cyto.a. 22342

MOV_Ag NPs.avi: Uptake dynamics (speeded up 1600x) showing different densities of Ag NP uptake by macrophages during $5 \mathrm{~h}$ exposure to $5 \mu \mathrm{M}$ Ag NP_BSA. Maximal intensity z-projections were made of sections in the 7-14 $\mu \mathrm{m}$ midrange subset. The red moving dots visible in the cytoplasm of cells indicate Ag NPs (also some extracellular). 


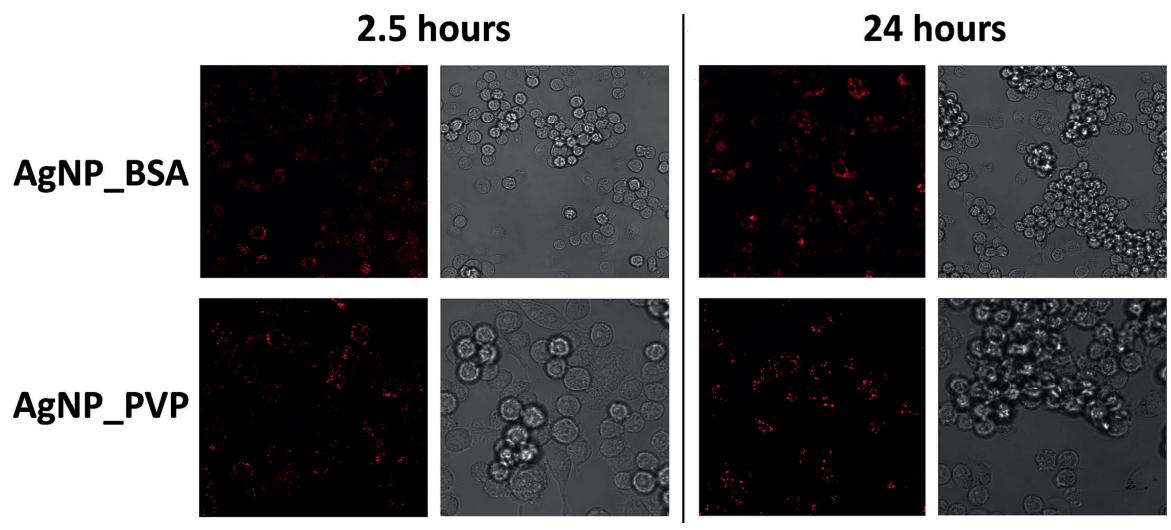

Figure S3. Confocal microscopic images of Ag NP uptake observed in RAW264.7 macrophage cells after 2.5 and $24 \mathrm{~h}$ exposure to $5 \mathrm{mg} / \mathrm{mL}$ Ag NPs. Upper row: Ag NP_BSA; lower row: Ag NP_PVP. Left column $2.5 \mathrm{~h}$ of exposure; Right column $24 \mathrm{~h}$ of exposure. Left panes: signals from Ag NPs; Right panes: bright-field micrographs of the same field. Size of all squares: BSA: $225^{\star} 225 \mu \mathrm{m}$; PVP: $112.5^{\star} 112.5 \mu \mathrm{m}$.

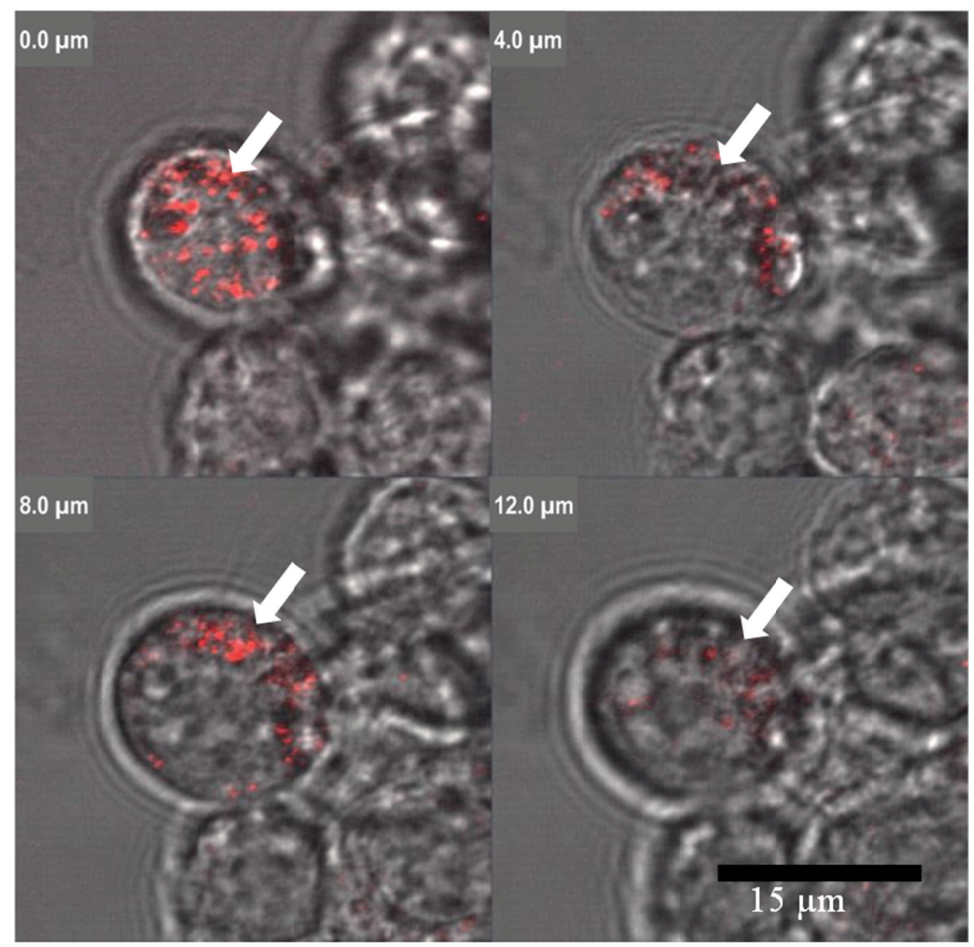

Figure S4. A subset of a $z$-series of a $15 \mu \mathrm{m}$ diameter macrophage with Ag NPs clusters in red throughout the cytoplasm (white arrows), excluding the nucleus. Cells were exposed for $22 \mathrm{~h}$ with negatively charged Ag NP_NC. Images display $1 \mu \mathrm{m}$ optical slices of Ag NP fluorescence as overlay on a (non-confocal) transmission image. 


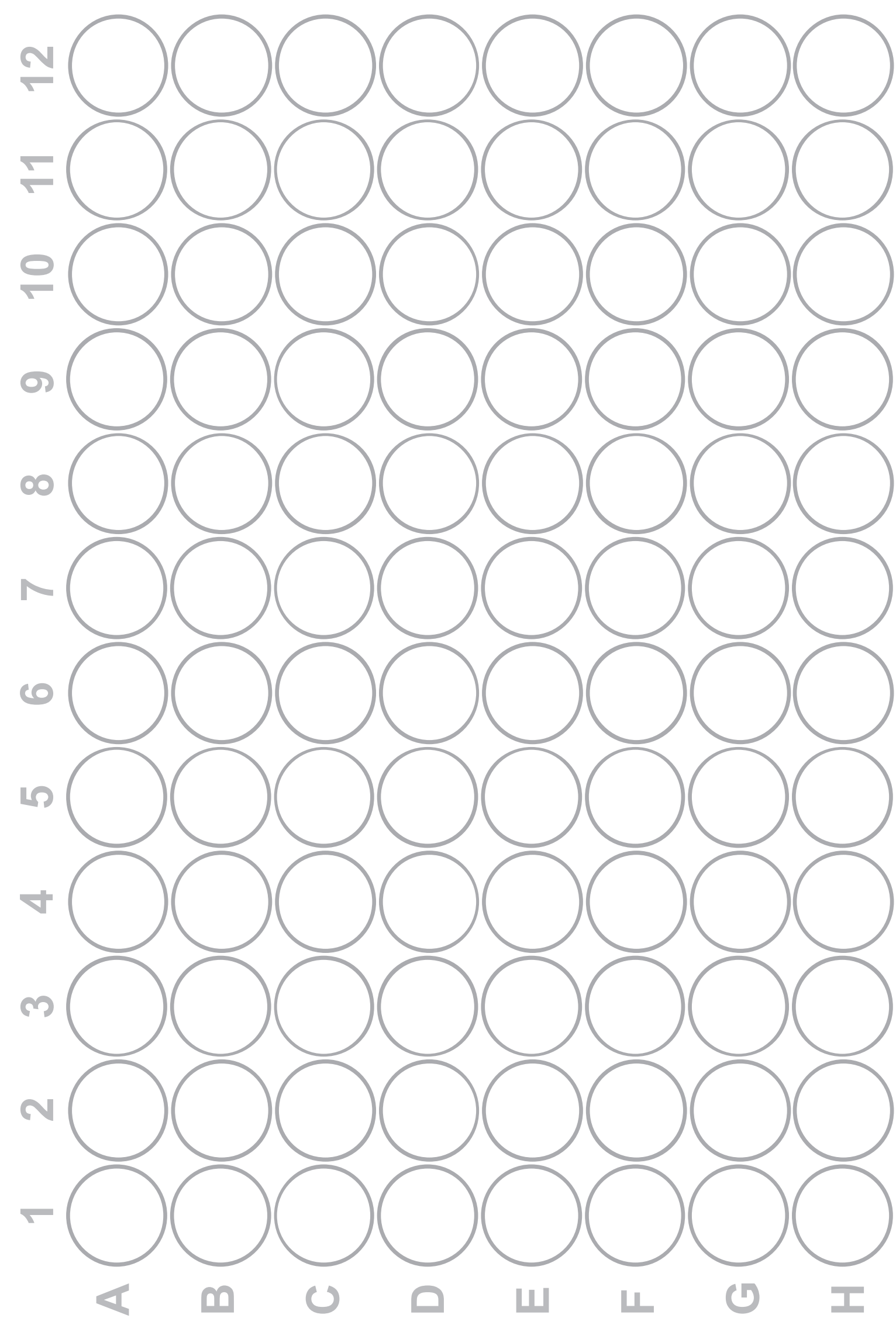




\section{Chapter 7}

General discussion, future perspectives and conclusions 


\section{GENERAL DISCUSSION}

The production of nanoparticles (NPs) has increased in the last decades and the number of products in which NPs are being incorporated is still growing. The rapid increase of nanotechnology has several benefits for society, yet there is an increasing concern that exposure to NPs may result in significant adverse health effects (Oberdorster et al. 2007, Hussain et al. 2015). Insight into the potential human health risks posed by NPs is essential for sustainable development and safe use of innovative products based on these materials. In the food sector NPs are used in food packaging materials and in food itself, including food supplements (Athinarayanan et al. 2015). Since NPs are incorporated in a variety of consumer products, it is likely that the general population gets exposed to NPs, via NP-containing products such as food and food packaging materials but also personal care products, textiles and medicines. In order to assess whether exposure to these NPs may pose a human health risk, NPs should be assessed for their safety. Testing all these NPs using in vivo animal experiments is undesirable for scientific, ethical and societal reasons. In order to reduce in vivo testing, alternative methods for testing of chemicals are currently being developed and validated (Eisenbrand et al. 2002, Van der Jagt et al. 2004, Stokes 2015). It would be desirable that the safety and risk assessment of NPs could be largely based on studies using in vitro models instead of in vivo models as this would reduce costs and time required to test the large numbers of NPs. Use of in vitro models may also help to define structure activity relationships and facilitate readacross within classes of NPs. Previous research has indicated that NP toxicity can be related to a certain extent to their physicochemical properties (Fourches et al. 2010). It would be of great value to get more insight in such relationships, to be able to set priorities for safety testing.

The aim of the present thesis was to investigate the potential of in vitro testing strategies to detect hazards of NPs, focusing on toxicokinetic as well as toxicodynamic endpoints. Toxicokinetic studies focused on translocation of NPs in in vitro models of the placental barrier, while toxicodynamic studies were directed at two endpoints that represent potential hazards of NPs that have not yet been well characterized including developmental toxicity and immunotoxicity. In the present thesis different types of NPs were used. Polystyrene nanoparticles (PS-NPs) were selected because of their commercial availability, with high quality and a wide variety of available physicochemical properties like surface charge, and fluorescent labeling enabling easy detection in toxicokinetic (translocation) studies. Several metal (oxide) NPs were selected as well, of which some are possible constituents of food additives like $\mathrm{TiO}_{2}$, $\mathrm{Fe}_{2} \mathrm{O}_{3}, \mathrm{SiO}_{2}$ and $\mathrm{Ag}$. Other metal oxide NPs that were selected were $\mathrm{Mn}_{2} \mathrm{O}_{3}, \mathrm{CuO}, \mathrm{Cr}_{2} \mathrm{O}_{3}, \mathrm{CoO}$ and $\mathrm{NiO}$ to which we may be exposed via products like paints, catalysts, construction materials, coatings and batteries.

Chapter 1 introduced the aim of the thesis and the various in vitro models and NPs used in the studies performed. Literature data indicate that translocation of NPs is possible across 
the gut, lung and skin barriers, related to oral, inhalation and dermal exposure, respectively (Landsiedel et al. 2012). In order to estimate the in vivo internal exposure, several in vitro models have been developed to study NP translocation across the lung, gut, skin and placental barrier (Mahler et al. 2012, Prow et al. 2012, George et al. 2015, Poulsen et al. 2015). Chapter 2 of the present thesis provided a review about the performance of such in vitro models for studies on toxicokinetics of NPs. The chapter described models that mimic the different barriers of the human body, with a focus on the lung, gut, skin and placental barrier, thus providing an overview of the state-of-the-art of using in vitro model systems, ranging from single cell-type monolayer to multi-cell (3D) models, to characterize the translocation of NPs across these different barriers. In this chapter it was concluded that at the current state-ofthe-art, the in vitro models do not yet allow to predict the absolute transfer rates but they do support the definition of relative transfer rates and can thus help to reduce animal testing by setting priorities for subsequent in vivo testing. The use of in vitro models is very promising since they are currently further improved to mimic the in vivo situation more closely by, for example, using co-cultures of different cell types and implementing them in a microfluidic chip format. When these models are further validated by testing exactly the same NPs in an in vivo set-up and in the in vitro model, they could be even used to determine the internal exposure (bioavailability) of NPs in an absolute way, besides to set priorities for NP toxicity testing.

Since part of the toxicodynamic studies of the present thesis were directed at developmental toxicity testing of NPs, an important toxicokinetic aspect studied in the present thesis in chapter 3 was placental translocation of NPs. In order to obtain insight in toxicity and translocation of NPs across the placental barrier, cytotoxicity and translocation was studied for one positively and two negatively charged PS-NPs of $50 \mathrm{~nm}$ in an in vitro model of the placenta. The model consisted of BeWo b30 cells, derived from a human choriocarcinoma grown on a transwell insert forming a cell layer that separates an apical from a basolateral compartment. PS-NPs were characterized with respect to size, surface charge, morphology and protein corona. In this study it appeared that in spite of similar size, surface charge and type of proteins in the protein corona, the differently charged NPs displayed a remarkable difference in cytotoxicity, with only the PS-NPs with an original positive charge inducing cytotoxicity. The relatively higher cytotoxicity of positively as compared to negatively charged NPs was reported before for tri-block copolymer NPs (Bhattacharjee et al. 2012) but also for other type of NPs like gold NPs (Liu et al. 2013) and silicon NPs (Bhattacharjee et al. 2013).

Translocation of PS-NPs appeared not to be related to PS-NP charge alone. A remarkable difference in translocation was found between the two $50 \mathrm{~nm}$ negatively charged PS-NPs that were obtained from different manufacturers. For the PS-NPs from Polysciences, the apparent permeability coefficient (Papp) was slightly higher than that of amoxicillin, a compound that is known to be translocated over the in vitro BeWo b30 placental barrier and the ex vivo 
placental barrier to only a limited extent (Li et al. 2013), with a Papp value of $13 \times 10^{-6} \mathrm{~cm} / \mathrm{s}$. When testing the negatively charged PS-NPs from Magsphere, no PS-NPs could be detected in the basolateral compartment upon adding a concentration of $10 \mu \mathrm{g} / \mathrm{ml}$ to the apical compartment and 24 hours incubation. This remarkable difference in translocation of the two similar sized negatively charged PS-NPs across the BeWo b30 placental barrier is in line with the 30-fold difference that was found in translocation of these two negatively charged PS-NPs across a Caco-2 monolayer in a similar transwell model system (Walczak et al. 2014). Since none of the characterized parameters, including size, surface charge and protein corona revealed remarkable differences between the two negatively charged NPs, the difference may originate from the chemical groups on the surface of the NPs generating the negative charge. The general conclusion from this study was that the in vitro BeWo b30 model can be used as a fast method to get an initial qualitative impression about the capacity of NPs to translocate across the placental barrier and to set priorities for further in vivo studies on translocation of NPs to the fetus.

Chapter 4 investigated whether the same PS-NPs as tested in chapter 3 for placental translocation, are able to cause in vitro developmental toxicity in the ES-D3 cell differentiation assay of the embryonic stem cell test (EST) focusing also on the effect that charge may have. The study showed that the two negatively charged PS-NPs did not show any effect in the ES-D3 cell differentiation assay up to the highest concentration tested while the positively charged PS-NP showed a concentration-dependent inhibition of ES-D3 cell differentiation. However, effect concentrations in the ES-D3 cell differentiation assay are close to cytotoxic concentrations, which indicated that the inhibition of the ES-D3 cell differentiation may be due to cytotoxic effects of the positively charged PS-NPs. This indicated that the inhibition of the ES-D3 cell differentiation by the positively charged PS-NPs may be caused by non-specific effects.

The ES-D3 cell differentiation assay is a useful tool to determine whether chemicals and NPs can pose a hazard for the embryonic development. However, to possibly become a risk for embryonic development, NPs need to be able to reach the developing embryo in the in vivo situation. Therefore, upon oral exposure, NPs would need to translocate across the intestinal barrier as well as the placental barrier. The bioavailability of many NPs upon oral exposure has been reported to be low, because of a limited translocation across the intestinal barrier (Walczak et al. 2014). However, in vivo studies using intravenous injection of NPs have shown that NPs can cause adverse effects to the developing embryo, indicating that NPs can translocate across the placenta in the in vivo situation (Hougaard et al. 2015). As indicated above in chapter 3, the potential of the same PS-NPs as tested in the EST was assessed to translocate across an in vitro placental barrier of human placental (BeWo) cells. The results of these studies indicated that the positively charged PS-NPs were hardly translocated across the BeWo cell layer, which could indicate that fetal exposure of these PS-NPs is expected to be 
low. Therefore, although the EST data indicated the potential of positively charged PS-NPs to cause developmental toxicity, such concentrations may not be reached in the in vivo situation because of the low placental transfer. On the other hand, the negatively Polysciences PS-NPs which did translocate across the BeWo barrier albeit to a limited extent, appeared not to cause an adverse effect in the in vitro developmental toxicity assay, also pointing to a limited hazard all together. These examples show that in order to predict which NPs may cause developmental toxicity in the in vivo situation, data obtained in the in vitro ES-D3 cell differentiation assay could be combined with placental translocation data from the BeWo model in order to select the NPs that are expected to have the highest potency in vivo. These estimations may then be used to select NPs that could be assessed for potential developmental toxicity in vivo. Combining the results from Chapter 3 and 4 allows the combination of kinetic considerations with the in vitro developmental toxicity assay to improve the prediction of the in vivo hazards as done previously by $\mathrm{Li}$ et al. ( $\mathrm{Li}$ et al. 2015, Li et al. 2015) for a series of azoles, for which the correlation of the prediction of in vivo developmental toxicity improved from $\mathrm{R}^{2}=0.57$ to $\mathrm{R}^{2}=0.95$ when the ES-D3 cell differentiation data were combined with the relative transport rates obtained in the BeWo model.

A literature search was performed on the reported potencies of other NPs in the ES-D3 cell differentiation assay, in order to assess whether effects of NPs in the ES-D3 cell differentiation assay may also be related to physicochemical properties other than surface charge, such as size or coating. Although the experiments in chapter 3 of the present thesis showed that positively charged PS-NPs are more toxic than negatively charged PS-NPs, it appeared that this may not be generalizable to other NPs. This follows from the fact that $\mathrm{SiO}_{2}, \mathrm{Ag}$ and $\mathrm{TiO}_{2} \mathrm{NPs}$ that were reported to inhibit ES-D3 cell differentiation were negatively charged, while the negatively charged PS-NPs of the present study did not affect ES-D3 cell differentiation. This implies that the negative charge did not arise from serum proteins which may affect the NP charge, as observed in the present study when NP charge was measured in medium with FCS. To obtain better insight in the role of NP surface charge on developmental toxicity more studies are required that assess the effects of NPs with different charge that are made of the same core material. The analysis of the available literature also indicated that the in vitro developmental toxicity potency of NPs may also largely depend on the coating applied. However, from the available data it cannot be concluded whether the toxicity potential is dependent on the coating alone, or whether it is also dependent on the combination of the core material and the coating. Therefore, although the limited data available indicate that charge, size and coating of NPs may be important characteristics that determine the developmental toxicity potential of NPs, more (systematic) studies are needed to assess how physicochemical characteristics of NPs relate to their developmental toxicity. This information may help to prioritize NPs for in vitro and in vivo developmental toxicity testing. 
In chapter 5, toxic effects of a series of metal (oxide) NPs were tested in macrophage RAW264.7 cells in order to obtain insight in effect of these NPs on cells of the innate immune response. In these macrophage RAW264.7 cells the effects of the metal (oxide) NPs were characterized on cell viability, TNF- $\alpha$ production and mitochondria-related parameters like production of reactive oxygen species (ROS), mitochondrial permeability transition pore (MPTP) opening, and intracellular ATP levels, since previous research indicated that mitochondria play an important role in NP-induced toxicity (Park et al. 2008, Sharma et al. 2011, Shvedova et al. 2012, Bhattacharjee et al. 2013). The results obtained indicated that $\mathrm{TiO}_{2}, \mathrm{Fe}_{2} \mathrm{O}_{3}, \mathrm{Cr}_{2} \mathrm{O}_{3}, \mathrm{CoO}$ and $\mathrm{NiO}$ NPs showed no effects on the selected endpoints up to the highest concentration tested $(200 \mu \mathrm{g} / \mathrm{ml})$, and that $\mathrm{SiO}_{2}$ and $\mathrm{Ag} \mathrm{NPs}$ caused concentration-dependent adverse effects in the RAW264.7 cells.

The results showed that $\mathrm{SiO}_{2}$ NPs were the most toxic ones among the tested food-related NPs. Exposure to food-related NPs is expected to be mainly via the gastrointestinal tract. When food products containing $\mathrm{SiO}_{2}$ were tested in an in vitro digestion model, simulating the conditions of the human gastrointestinal tract, it was found that $\mathrm{SiO}_{2} \mathrm{NPs}(5-200 \mathrm{~nm})$ were still present in the intestinal content (Peters et al. 2012). This indicates that it is most likely that upon consumption of foods containing particulate $\mathrm{SiO}_{2}$, the gut epithelium is exposed to $\mathrm{SiO}_{2} \mathrm{NPs}(5-200 \mathrm{~nm}$ ) (Peters et al. 2012). To enter the systemic circulation, NPs need to be absorbed in the gastrointestinal tract. In an in vivo study in rats it was shown that $6.6-9.7 \%$ of both $\mathrm{SiO}_{2} \mathrm{NPs}$ of 20 and $100 \mathrm{~nm}$ were absorbed into the systemic circulation, with no significant effect of the particle size, when orally administered at a single dose of 500 and $1000 \mathrm{mg} /$ $\mathrm{kg}$ (Lee et al. 2014), indicating that $\mathrm{SiO}_{2}$ NPs can get translocated across the intestinal barrier.

With an estimated daily intake of $\mathrm{SiO}_{2}$ in the nano-size range of $1.8 \mathrm{mg} / \mathrm{kg}$ bw/day (van der Zande et al. 2014, van Kesteren et al. 2014), the daily intake of a $70 \mathrm{~kg}$ person would be 126 $\mathrm{mg}$. In the adult human the gastrointestinal tract represents a considerable surface area of about $200 \mathrm{~m}^{2}$ which has the potential to interact with ingested NPs (Bergin et al. 2013). In addition the gastrointestinal tract has localised representations of the adaptive immune system in the form of gut-associated lymphoid tissue (GALT) aggregates (Peyer's Patches) (Bergin and Witzmann 2013). These Peyer's patches account for $1 \%$ of the total surface area of the gastrointestinal tract (des Rieux et al. 2006). About 9 litres of fluid pass through the gastrointestinal tract each day (Sherwood L. 2014). Thus, upon oral intake of $126 \mathrm{mg} \mathrm{SiO}{ }_{2}$ per day this would lead to a concentration of $14 \mu \mathrm{g} / \mathrm{ml}$, assuming full distribution of the NPs over the entire intestinal content, which is lower than the lowest observed adverse effect concentration of $100 \mu \mathrm{g} / \mathrm{mL}$ in the present study. Only when using a worst case assumption of only limited dilution of an oral dose of $126 \mathrm{mg} \mathrm{SiO}{ }_{2} \mathrm{NPs}_{\text {in }}$ the human intestine, local concentrations can be expected to reach the effect levels observed in vitro. 
Furthermore, if $100 \%$ of the estimated daily intake of $\mathrm{SiO}_{2} \mathrm{NPs}(126 \mathrm{mg})$ would be transported from the intestines into the blood, assuming 5 liters of blood and a 100\% clearance per day, a maximal concentration of $25 \mu \mathrm{g} / \mathrm{ml}$ would be reached if NPs would not distribute to tissues. Also this concentration is lower than the lowest observed adverse effect concentration of 100 $\mu \mathrm{g} / \mathrm{mL}$ defined in the present thesis. Considering that the oral bioavailability is lower than $10 \%$ and that NPs also distribute to tissues (Lee et al. 2014), concentrations higher than 2.5 $\mu \mathrm{g} / \mathrm{mL}$ are not likely to be reached in a real-life exposure scenario. This concentration is far below the concentrations of $\mathrm{SiO}_{2}$ causing in vitro toxicity in the present study, suggesting that in vivo toxic effects of food additive related $\mathrm{SiO}_{2} \mathrm{NPs}$ on macrophages, are expected to be limited. This is in line with the results from an oral repeated-dose toxicity study in which male Wistar rats were exposed to $\mathrm{SiO}_{2} \mathrm{NPs}$ via the diet for 28 or 84 days, showing no toxicity even at the highest dose level tested (2500 mg/kg bw/day) (van der Zande et al. 2014).

It has to be kept in mind that NPs may not only affect the gastrointestinal cells directly but may also influence the microbiome although the evaluation of effects of NPs on the microbiome is in its early infancy (Pietroiusti et al. 2015). For example, the cytotoxicity of Ag NPs on Escherichia coli as representative for intestinal bacteria was compared with the cytotoxicity of Ag NPs on gastrointestinal cells. This revealed that Ag NPs affected bacteria at lower concentrations than the gastrointestinal cells indicating that Ag NPs may cause adverse effects by selectively affecting the gut microbiota (Frohlich et al. 2016). In a study using an in vitro colon model (Taylor et al. 2015), exposure to $\mathrm{TiO}_{2}, \mathrm{ZnO}$ and $\mathrm{CeO}_{2} \mathrm{NPs}$ induced significant phenotype changes of the gut microbial community, including effects on the production of short chain fatty acids which are the key metabolites in the control of energy homeostasis and have been implicated in the development of obesity (Schwiertz et al. 2010). For $\mathrm{SiO}_{2}$ and other food-related NPs, however, the occurrence of such effects remains to be investigated.

Altogether, results obtained in chapter 5 showed no or limited effects of the NP formulations of metal (oxide) food additives on cell viability, ROS production, MPTP opening, ATP levels and TNF- $\alpha$ production in RAW264.7 macrophages. Effects were only observed at high concentrations that may not be physiologically relevant, indicating that related adverse effects upon exposure to the respective NPs in vivo may be limited.

Of the NPs tested in chapter 5, Ag NPs were among the NPs showing most effects. The volume of Ag NPs-based products entering the market is relatively high, making the risk assessment of Ag NPs a priority. Therefore to elucidate the factors that drive Ag NPs' potential to pose health risks, chapter 6 assessed the effects of specifically synthetized Ag NPs with two different sizes $(20$ and $50 \mathrm{~nm}$ ) and with three different surface coatings (chitosan: $\mathrm{Ag}$ NP_Chit, bovine serum albumin: Ag NP_BSA, polyvinylpyrrolidone: Ag NP_PVP) in the same in vitro RAW264.7 model. The effect of surface charge and size of NPs was character- 
ized on cell viability, TNF- $\alpha$ production, ROS production, MPTP opening, and intracellular ATP levels. Decreased cell viability was observed for all Ag NPs tested. The results from MTT, ATP and MPTP opening assays showed that adverse effects from exposure to the tested Ag NPs on these endpoints were similar irrespective of type or size. However, the role of surface coating/charge in influencing TNF- $\alpha$ induction was demonstrated by the fact that only negatively charged Ag NP_BSA induced TNF- $\alpha$ production (80x higher than control). Further, significant ROS induction was only observed with the $20 \mathrm{~nm}$ positively charged Ag NP_Chit. Effect of size was less prominent under the experimental conditions, showing only minimal differences that were not statistically significant. Overall, the negatively charged Ag NP_BSA appeared to be most potent in inducing adverse effects on the macrophages. This toxicity of the negatively charged Ag NPs is the opposite of what was found in chapter 3 for the PS-NPs, since the positively charged PS-NPs appeared to be the most cytotoxic to BeWo cells. So this points again to the direction that the effect of surface charge may vary with the type of NPs investigated, indicating that more studies with other NPs with different core material and with different charges are required. One has to keep in mind that Ag NPs are known to release ions which can also contribute to toxicity even if the Ag NPs has a negative surface charge, and in general the smaller the Ag NP the higher the ion release (Johnston et al. 2010, Reidy et al. 2013). Given this ion release, the PS-NPs used in chapter 3 and 4 seem to be a better model to evaluate the effect of charge or size since they do not present these additional factors leading to toxicity.

Taken together, the present thesis provided further evidence of the influence of physicochemical properties of NPs in driving toxicity in an in vitro model. However, the determination of the fate and toxicity of NPs using in vitro or in vivo models is a challenge that needs further evaluation. A combination of several factors likely play a role in determining the outcome of exposure including factors like NP core material and presence and type of coating agents resulting in various physicochemical properties (size, charge, etc.), as well as the toxicity test models used and end-points studied. This has been observed in earlier reviews (Johnston et al. 2010, Reidy et al. 2013) and appears to hamper conclusive evaluation of the role of physicochemical characteristics of NPs in the modes of action underlying their potential hazards and risks so far.

The results obtained do show however that in vitro assays can detect differences in potential hazards posed by NPs. Therefore it is concluded that the results of the work presented in this thesis will contribute to the further development and use of non-animal based testing strategies for safety testing of NPs providing insight into selected potential hazards of the tested NPs. 


\section{FUTURE PERSPECTIVES}

Although the present thesis has extended the understanding and prediction of potential hazards of different NPs, several issues remain to be elucidated to fully understand the hazards of NPs to human health and the environment. The current section presents some considerations on future topics of importance in this field. These topics include:

- Use of alternative testing strategies and especially the EST to detect developmental toxicity.

- Physicochemical characterization of NPs

- Predictive toxicology: Nano-QSAR models

- Dosimetry

- The role of the microbiome

Use of alternative testing strategies and especially the EST to detect developmental toxicity. Given the high number of NPs for which at present in vivo toxicity data are missing the potential to use alternative in vitro testing methods to detect possible health hazards of NPs, is of high importance to set priorities in future safety testing and also to support development of safer NPs. An important endpoint to include in these activities is developmental toxicity since this is an endpoint requiring relatively large numbers of experimental animals (Rovida et al. 2009). In the present thesis the ES-D3 cell differentiation assay of the EST was used. The main advantage of this assay over other alternative assays for developmental toxicity testing is that it does not require the use of any animals because it uses an immortal cell line isolated from a mouse blastocyst. The EST is also relatively easy to carry out and the endpoints used in the assay do not require extensive knowledge on morphologic development (de Jong et al. 2011).

However, the differentiation of the stem cells into beating cardiomyocytes as only endpoint might not be sufficient for determination of all possible embryotoxic effects (zur Nieden et al. 2004). With the EST, only the effects on mesodermal development are recorded. But some substances and also NPs might have embryotoxic effects on specific cell-types other than cardiomyocytes. This may lead to false negative results if the test compound affects cell types other than the myocardium. Therefore, also the differentiation into neural and endothelial cells, chondrocytes and osteoblasts should be investigated (Buesen et al. 2004).

Other alternative assays for in vivo developmental toxicity are the rat Whole Embryo Culture (WEC) and the limb bud micromass (MM) test (Genschow et al. 2000, Augustine-Rauch et al. 2010). The WEC is used to assess the effects of chemicals on the development of the complete embryo within its intact visceral yolk sac outside the uterus, during a critical phase in organogenesis (de Jong et al. 2011). The test makes use of mammalian embryos, but both the 
exposure and the assessment of effects occur in vitro (Piersma et al. 2008). Therefore, WEC systems offer the most complete in vitro alternative for animal testing for developmental toxicity. The test does require animal material, but has the advantage of representing embryogenesis in full complexity from cellular proliferation and differentiation to pattern formation (Piersma 2004).

In the MM test, chondrogenesis of mesenchymal cells of limb buds of rat embryos is studied (Kistler 1987). The test involves exposing cultured chick, mouse or rat embryo midbrain or limb cells to test agents for varying times and estimating the inhibition of cell differentiation in these cultures. The MM assay reported that when cells from the undifferentiated mesenchyme of early chick embryo limbs are cultured in small volumes at high density, they formed numerous small aggregates (foci) of cells of differentiating chondrocytes within a background of apparently undifferentiated cells (Flint 1993). In principle, the MM developmental toxicity test is based on detecting the ability of a particular compound to inhibit the formation of foci. Embryotoxic compounds will reduce the number of foci or the number of cells within foci (Spezia et al. 2013). Both the WEC and MM assay rely on a constant source of embryonic tissues from pregnant animals. However the MM assay is considered most useful for identifying strongly embrytotoxic compounds (Genschow et al. 2004) since the predictivity for non-embryotoxic compounds and the precision for weakly embryotoxic compounds were judged to be insufficient.

Another in vitro test for embryotoxicity is the so-called chick embryotoxicity screening test (CHEST) assay. The main endpoints assessed using the CHEST are mortality, malformations, embryo development, blood vessel development and blood vessel coloration. The test compounds can easily be administered to the eggs and effects on the developing embryo can be investigated. However, the CHEST has been criticised for not being able to distinguish general toxicity from specific developmental effects and the absence of mammalian maternal-foetal relations (Jelinek et al. 1985).

A final possible method to study in vitro developmental toxicity is the Zebrafish Embryotoxicity Test (ZET). This test uses non-mammalian embryos. The effects of compounds are tested on the development of embryos of the zebrafish. In principle, the fertilized fish eggs are exposed to different concentrations of a test substance. At different time points, the exposed developing fish embryos are observed and scored for lethal and embryotoxic effects. The ZET has been used for testing embryotoxiciy of Ag NPs (van Aerle et al. 2013, Yoo et al. 2016), $\mathrm{ZnO}, \mathrm{SiO}_{2}, \mathrm{Al}_{2} \mathrm{O}_{3}$, Pt and Au NPs (George et al. 2011) and $\mathrm{TiO}_{2} \mathrm{NPs}$ (Brundo et al. 2016, Shih et al. 2016). Yoo et al. 2016 investigated embryotoxicity effects of $10 \mathrm{~nm} \mathrm{Ag} \mathrm{NPs} \mathrm{during}$ zebrafish development, using the following parameters: abnormal morphology, mortality, hatching rate and heart rate. The hatching rate differed not significantly between the exposure 
groups while the Ag NP exposed group showed increased mortality (11\%) and teratogenicity (15\%) at $72 \mathrm{~h}$ compared to the control group for which these values amounted to $2 \%$ and $0 \%$ respectively. The heart rate of Ag NP exposed embryos tended to be lower than that of the control group but differences were not statistically significant (Yoo et al. 2016). Van Aerle et al. (2013) investigated the effects of exposure to bulk Ag, $10 \mathrm{~nm} \mathrm{Ag} \mathrm{NPs} \mathrm{or} \mathrm{ionic} \mathrm{Ag} \mathrm{to} \mathrm{ze-}$ brafish embyros using a Next Generation Sequencing approach. All treatments affected gene expression and those genes associated with oxidative phosphorylation and protein synthesis overlapped strongly between the three treatments indicating similar mechanisms of toxicity for the three forms of silver studied (van Aerle et al. 2013).

One study investigated a batch of commercial metal/metal oxide NPs both for their cytotoxic potential in vitro using automated epifluorescence microscopy on mammalian BEAS-2B and RAW 264.7 cells and in vivo using the zebrafish (George et al. 2011). The automated epifluorescence procedure measures sub-lethal (e.g. intracellular $\mathrm{Ca}^{2+}$ flux and mitochondrial perturbations) and lethal (cell death with increased membrane permeability) responses. Among the NPs analyzed, $\mathrm{Pt}, \mathrm{Ag}, \mathrm{SiO}_{2}, \mathrm{Al}_{2} \mathrm{O}_{3}$, and $\mathrm{Au}$ NPs triggered sub-lethal effects without cytotoxicity. Quantum Dots (QDs) and ZnO NPs triggered sub-lethal effects and cytotoxicity. In order to compare the in vitro with the in vivo response outcomes in zebrafish embryos, NPs were tested for their impact on mortality rate, hatching rate, cardiac rate, and morphological defects. While QDs, ZnO and Ag NPs induced morphological abnormalities or interfered with embryo hatching, Pt and Ag NPs exerted inhibitory effects on cardiac rate at the sub-lethal dose of $15 \mu \mathrm{g} / \mathrm{mL}$. Ag NP-treated embryos developed bent spines, short body sizes, pericardial edema, submandibular edema and bent tails at $120 \mathrm{hrs,} \mathrm{while} \mathrm{the} \mathrm{majority} \mathrm{of} \mathrm{ZnO}$-treated embryos remained unhatched and embryos treated with QDs died early and underwent complete dissolution within 24 hrs. Ag NP was clearly more toxic in zebrafish embryos than towards mammalian cells in vitro (George et al. 2011).

\section{Physicochemical characterization of NPs}

The ability to create NPs with a wide variety of characteristics with respect to chemistry, shape, size and surface properties led to attention for the nanotoxicological aspects regarding of the physicochemical characteristics of NPs (Westmeier et al. 2016). It is well known that the biological activity of NPs depends on their physicochemical parameters including size, shape, surface area, surface charge, crystal structure, chemical composition and surface chemistry (Fadeel et al. 2015). Generally, by using the currently available methods, it is only possible to measure a single or several characteristic(s) of NPs but not all (Bouwmeester et al. 2011). Investigations of the NPs behaviour in physiological media are very important to interpret the toxicological results. As an example the formation of a protein corona formation can cause changes in charge, size and surface chemistry of a certain NP thereby impacting on its uptake and cellular fate (Corbo et al. 2016). Therefore, physicochemical characteristics and biological 
effects should preferably be measured under the same experimental conditions (Meissner et al. 2009).

In this thesis NPs were characterized in the medium used for the respective assay using SEM and dynamic light scattering (DLS) for size and zeta potential (for surface charge). Chapter 3 pointed out that only size and charge characterization of PS-NPs were not enough for interpreting the results. Translocation of the different PS-NPs across the BeWo cell layer was not found to be related to PS-NP charge and a remarkable difference in translocation was found between the two $50 \mathrm{~nm}$ negatively charged PS-NPs from different manufacturers, where one did not translocate at all to the basolateral compartment. Therefore the protein corona was also assessed using SDS-PAGE. Biomolecules rapidly adsorb to NPs as they have a high surface energy, leading to the formation of the protein corona which critically affects the NPs biological identity (Cedervall et al. 2007). The protein corona formation can influence the delivery properties of NPs, as well as their toxicity (Tenzer et al. 2013) and plays a critical role in cellular uptake (Pelaz et al.,2015), accumulation, biodegradation and clearance of NPs (Saptarshiet al., 2013). Protein corona analyses of the 3 different PS-NPs in chapter 3 revealed differences in the amount but not the nature of the proteins in the corona of the three different PS-NPs. Since none of the characterized parameters, including size, zeta potential and protein corona revealed remarkable differences between the two negatively charged NPs, it was argued that the difference may originate from the chemical groups on the surface of the NPs generating the negative charge. Previously it was demonstrated that three different positively charged tri-block copolymer nanoparticles showed different cellular uptake and toxicity in spite of a similar overall size and zeta potential, and that only a different shielding of the charge due to subtle differences in the charge generating surface moiety influenced the cellular uptake and toxicity (Bhattacharjee et al. 2011). Such observations indicate that by characterising size, zeta potential and protein corona, even when done in the relevant medium or body fluid, may prove insufficient for full characterization of the NP parameters that direct the biological effects and potential toxicity. Obviously this also hampers definition of (Q)SARs and read-across and suggests that at the current state-of-the-art parameters generally used to characterise NPs physicochemical characteristics may prove insufficient to understand the differences in their toxicity.

Although techniques for characterization of NPs have progressed greatly over the past 10 years, further improvements are highly recommended. For example it would be advantageous to modify current standards such as DLS and TEM/SEM in order to perform them with a higher sensitivity and in a more relevant biological environment (Hussain et al. 2015). Limitations of the DLS method include that the particle size is correlated with the light scattered and the presence of a few large particles, for example from the culture medium or relevant biological fluid, could mask the presence of a high number of small particles (Handy et al. 2008). 
Establishing nano-QSARs linking NP/corona properties to (patho)physiological responses remains a still distant goal despite the achievements of the past years. It is clear that characterizing the nano-cellular interactions is a critical step in the development of a fundamental understanding of nanotoxicology. International guidelines for physicochemical characterization are needed for regulatory purposes, and the methods used for characterization need to be standardized and validated (Fadeel et al. 2015).

\section{Predictive toxicology: Nano-(Q)SAR models}

Because of the high costs and time involved in toxicological in vivo and in vitro studies, there has been considerable interest in computer based strategies defining alternative computational methods, like the application of (quantitative) structure-activity relationship ((Q)SAR) models. (Q)SAR models are theoretical models that relate the structure or physicochemical characteristics of compounds to their biological activities. (Q)SAR models could be a useful screening tool, eliminating the need to test every single NP on an individual basis (Oksel et al. 2015). Considering the large number of factors that differ from conventional chemicals and that are likely to influence the biological activity of NPs, it might also be concluded that traditional (Q)SAR approaches need serious reconsiderations in order to be applied to NPs (Oksel et al. 2015). The simple application of classic (Q)SAR approaches based on the representation of various substances by the structure of their molecules is impossible for the majority of NPs (Toropova et al. 2016). Because of the high structural complexity and diversity of NPs, it is difficult to develop quantitative parameters capable of characterizing the structural and chemical properties of NPs. In addition, systematic studies that assess physicochemical and structural properties in relation to biological effects of NPs are scarce, making the development of predictive computational models and their validation difficult, since these procedures require relatively large amounts of data (Fourches et al. 2010). Moreover, due to the fact that large datasets are not available for a large part of the NPs one might need to develop a methodology that provides predictions based on smaller datasets. Few studies have reported on the development of nano-(Q)SARs. Recently, an approach was published to develop a (Q) SAR for fullerene NPs based on a small dataset (Toropova et al. 2016). In this study a QSAR was developed based on several data (conditions) related to (i) dose (g/plate); (ii) metabolic activation (with or without mix S9); and (iii) illumination (dark or irradiation) as a tool to predict mutagenicity of fullerene NPs in bacterial strains. These computational experiments have shown that the described approach, based on a list of several conditions and not on molecular structure as is usually done in the classical approach, can be a tool to build up models for the prediction of mutagenicity of fullerene NPs under different conditions, based on a small dataset.

Burello et al. 2011, have proposed a theoretical framework to predict the oxidative stress potential of oxide NPs based on the available electronic energy levels in the NPs (Burello et al. 
2011). Fourches et al. 2010 investigated the potential of (Q)SAR modelling to establish statistically significant relationships between measured biological activity profiles of NPs and their physical, chemical, and geometrical properties, either measured experimentally or computed from the structure of NPs (Fourches et al. 2010). They studied a first dataset of 51 NPs with different metal cores which were tested in four cell lines (monocytes, hepatocytes, endothelial and smooth muscle cells) in four different assays (ATP levels, reducing equivalents, caspase mediated apoptosis and mitochondrial membrane potential). It was demonstrated that NPs with similar biological activity can be also recognized as similar by their type of metal core, since NPs in one cluster featured the same metal core, independently of their surface modifiers. In a second dataset they studied 109 NPs with the same core but with different surface modifications for their cell uptake activity. Here the structure of organic small molecules conjugated to the NP surface was the only difference between the 109 NPs. Factors that correlated with cellular uptake were: lipophilicity, molecular refractivity, specific Van der Waals surface area and electrostatic descriptors. These findings imply that the cellular behaviour of a NP dataset based on a common core can be predicted using (Q)SAR analysis of the surface modifying ligands (Fourches et al. 2010).

At the moment the research for nano-(Q)SAR models continues. There remains the need for thorough testing and validation of $(\mathrm{Q}) \mathrm{SAR}$ methodologies, and there are still barriers that need to be overcome to establish predictive, reliable, and legally acceptable nano-(Q)SAR models. A main issue is the scarcity of consistent and high-quality experimental data which are needed to develop a robust and predictive nano-(Q)SAR model (Oksel et al. 2015). The scarcity of such data is mainly caused by difficulties in standardizing nanotoxicity testing methodologies and characterization conditions. High quality data are required, which are consistent and systematically obtained for NPs that have been comprehensively characterised under relevant exposure conditions prior to toxicological testing (Oksel et al. 2015). It is therefore suggested that, due to high variability in the molecular structures and different mechanisms of toxicity, individual classes of NPs should be modelled separately (Puzyn et al. 2009). The establishment of standard protocols is essential to enable accurate measurements of physicochemical and biological properties of NPs (Oksel et al. 2015).

\section{Dosimetry}

Another challenge is to arrive at a consensus about which dose metric is best to be used in nanotoxicological studies, for example mass, particle number or surface area (Hussain et al. 2015). Dosimetry is the accurate description of the dose or the amount of NPs that comes into contact with the biological target. It is an important aspect to take into account when predicting risks but it should be measurable and it should be possible to incorporate it in regulations. Currently the dose is often defined as the nominal dose, so the amount of NPs introduced into the culture medium expressed in mass per volume $(\mu \mathrm{g} / \mathrm{ml})$. However, it is also possible 
to measure the intracellular or deposited doses (Fadeel et al. 2015). It has been suggested that the NP surface area could be more relevant in nanotoxicology studies than the mass unit (Oberdorster et al. 2007). Braakhuis et al. have demonstrated that for short-term inhalation of Ag NPs, the total surface area of Ag NPs that reach the alveoli seems the most suitable dose descriptor for the acute effects of Ag NPs in a range of sizes (Braakhuis et al. 2016). This is because the alveoli are the site of the observed toxicity and Ag NPs can release Ag ions, which possibly cause the observed effects. The release of Ag ions increases with decreasing particle size due to their relative larger surface area (Braakhuis et al. 2016). This indicates that for other types of NPs that do not release ions the suitable dose descriptor could be different. There is probably no one-size-fits-all dose metric for NPs and various approaches depending on the type of NP and type of toxicological testing system might be needed to apply.

So dosimetry in nanotoxicological studies is complicated by additional topics which are unique for NPs. For example NPs such as $\mathrm{Cu}$ and Ag release ions over time thereby introducing a second factor of interaction with the cells. In addition, NPs that are insoluble may have an influence on biokinetic behavior, since there is an increase potential for bioaccumulation, which makes it difficult to estimate a delivered dose at specific tissues or cells. Insoluble NPs may cause a diverse range of chronic effects and have the potential to reside for years in the respiratory tract (Bakand et al. 2016). Also the fact that the properties of NPs may vary significantly between cell culture media and physiological fluids may influence the dose metric that most adequately describes the exposure. For example, the protein corona that forms on NP surface when in it comes in contact with biological fluids such as serum, mediates the interactions with cells and could affect the cellular uptake of NPs. A study found that SiO2 NPs exposed to cells in the absence of serum have a stronger adhesion to the cell membrane and a higher uptake in cells, compared to SiO2 NPs exposed to cells in medium containing serum, when a preformed corona is present on their surface (Lesniak et al. 2012). This indicates the importance of proper performance and description of the physicochemical characterization of nanoparticles in every experiment.

\section{The role of the microbiome}

The gut microbiome consists of trillions of microorganisms located in our gastrointestinal tract (Tsai et al. 2009). The gut microbiome plays a major role in influencing the health status of the host. There is a complex interaction between the gut microbiome, diet, genetics, environmental influence, host health and neurotransmitters and metabolites (Ghaisas et al. 2015, Pietroiusti et al. 2015). Gut bacteria play a major role in immune modulation and development of the nervous system and are the main source of vitamin $\mathrm{K}$ and $\mathrm{B}$ complex. Alterations in the microbiome have been suggested to play a role in several diseases like irritable bowel syndrome (IBS), ulcerative colitis, Alzheimer's disease, Parkinson's disease, type-2 diabetes, multiple sclerosis and autism (Althani et al. 2015, Ghaisas et al. 2015). 
The evaluation of effects of NPs on the microbiome is in its early infancy. Altough data are limited, available experimental data suggest that different types of NPs including carbon nanotubes (CNTs), $\mathrm{TiO}_{2}, \mathrm{CeO}_{2}, \mathrm{ZnO}, \mathrm{SiO}_{2}$ and $\mathrm{Ag} \mathrm{NPs}$, may affect the microbiome in a way that may result in adverse health effects. It is speculated that this may then result in clinical disorders such as colitis, obesity and immunological dysfunctions (Pietroiusti et al. 2015). For example in a study using an in vitro colon model (Taylor et al. 2015) which included the microbial community taken from a young healthy donor, exposure to $\mathrm{TiO}_{2}$ and $\mathrm{ZnO}$ NPs induced changes in various phenotypic traits of the colonic bacteria, including the production of short chain fatty acid which are the key metablolites in the control of energy homeostasis and have been implicated in the development of obesity (Schwiertz et al. 2010). Development of colitis associated to gut microbiota alterations was reported in mice which were exposed to ambient particulate matter containing carbon based NPs (Kish et al. 2013). Given the few studies done so far on possible microbiome-NP interactions, there is a need for more in vitro and in vivo studies. In these studies the potential degradation of ingested NPs by the microbiome could be investigated as well (Pietroiusti, Magrini et al. 2015).

\section{OVERALL CONCLUSION}

Overall, the work presented in this thesis showed that in vitro assays can contribute to the development and use of non-animal based testing strategies for safety testing of NPs and provide insight into selected potential hazards of the tested NPs and a means to set priorities in future in vivo tetsing. Use of in vitro models may also help to define nano-(Q)SAR methodologies. The work presented in this thesis also showed that the role of surface charge varies with the type of NP and the endpoint of interest. So this would mean that case-by-case testing is needed to which in vitro assays will form an important contribution. 


\section{REFERENCES}

Althani, A., H. E. Marei, W. S. Hamdi, G. K. Nasrallah, M. E. El Zowalaty, S. Al Khdor, M. Al-Asmakh and H. Abdel-Aziz (2015). "Human Microbiome and Its Association With Health and Diseases." Journal of cellular physiology.

Athinarayanan, J., A. A. Alshatwi, V. S. Periasamy and A. A. Al-Warthan (2015). "Identification of Nanoscale Ingredients in Commercial Food Products and their Induction of Mitochondrially Mediated Cytotoxic Effects on Human Mesenchymal Stem Cells.” J Food Sci 80(2): N459-464.

Augustine-Rauch, K., C. X. Zhang and J. M. Panzica-Kelly (2010). "In vitro developmental toxicology assays: A review of the state of the science of rodent and zebrafish whole embryo culture and embryonic stem cell assays." Birth defects research. Part C, Embryo today : reviews 90(2): 87-98.

Bakand, S. and A. Hayes (2016). "Toxicological Considerations, Toxicity Assessment, and Risk Management of Inhaled Nanoparticles." International journal of molecular sciences 17(6).

Bergin, I. L. and F. A. Witzmann (2013). "Nanoparticle toxicity by the gastrointestinal route: evidence and knowledge gaps." International journal of biomedical nanoscience and nanotechnology 3(1-2).

Bhattacharjee, S., D. Ershov, K. Fytianos, J. Gucht, G. Alink, I. Rietjens, A. Marcelis and H. Zuilhof (2012). "Cytotoxicity and cellular uptake of tri-block copolymer nanoparticles with different size and surface characteristics." Particle and Fibre Toxicology 9(1): 11.

Bhattacharjee, S., D. Ershov, J. V. Gucht, G. M. Alink, I. M. Rietjens, H. Zuilhof and A. T. Marcelis (2011). "Surface charge-specific cytotoxicity and cellular uptake of tri-block copolymer nanoparticles." Nanotoxicology.

Bhattacharjee, S., I. M. Rietjens, M. P. Singh, T. M. Atkins, T. K. Purkait, Z. Xu, S. Regli, A. Shukaliak, R. J. Clark, B. S. Mitchell, G. M. Alink, A. T. Marcelis, M. J. Fink, J. G. Veinot, S. M. Kauzlarich and H. Zuilhof (2013). "Cytotoxicity of surface-functionalized silicon and germanium nanoparticles: the dominant role of surface charges." Nanoscale 5(11): 4870-4883.

Bouwmeester, H., I. Lynch, H. J. Marvin, K. A. Dawson, M. Berges, D. Braguer, H. J. Byrne, A. Casey, G. Chambers, M. J. Clift, G. Elia, T. F. Fernandes, L. B. Fjellsbo, P. Hatto, L. Juillerat, C. Klein, W. G. Kreyling, C. Nickel, M. Riediker and V. Stone (2011). "Minimal analytical characterization of engineered nanomaterials needed for hazard assessment in biological matrices." Nanotoxicology 5(1): 1-11.

Braakhuis, H. M., F. R. Cassee, P. H. Fokkens, L. J. de la Fonteyne, A. G. Oomen, P. Krystek, W. H. de Jong, H. van Loveren and M. V. Park (2016). "Identification of the appropriate dose metric for pulmonary inflammation of silver nanoparticles in an inhalation toxicity study." Nanotoxicology 10(1): 63-73.

Brundo, M. V., R. Pecoraro, F. Marino, A. Salvaggio, D. Tibullo, S. Saccone, V. Bramanti, M. A. Buccheri, G. Impellizzeri, V. Scuderi, M. Zimbone and V. Privitera (2016). “Toxicity Evaluation of New Engineered Nanomaterials in Zebrafish." Frontiers in physiology 7: 130.

Buesen, R., A. Visan, E. Genschow, B. Slawik, H. Spielmann and A. Seiler (2004). "Trends in improving the embryonic stem cell test (EST): an overview." Altex 21(1): 15-22.

Burello, E. and A. P. Worth (2011). "A theoretical framework for predicting the oxidative stress potential of oxide nanoparticles.” Nanotoxicology 5(2): 228-235.

Cedervall, T., I. Lynch, M. Foy, T. Berggard, S. C. Donnelly, G. Cagney, S. Linse and K. A. Dawson (2007). "Detailed identification of plasma proteins adsorbed on copolymer nanoparticles." Angew Chem Int Ed Engl 46(30): 5754-5756.

Corbo, C., R. Molinaro, A. Parodi, N. E. Toledano Furman, F. Salvatore and E. Tasciotti (2016). "The impact of nanoparticle protein corona on cytotoxicity, immunotoxicity and target drug delivery." Nanomedicine (Lond) 11(1): 81-100.

de Jong, E., M. Barenys, S. A. B. Hermsen, A. Verhoef, B. C. Ossendorp, J. G. M. Bessems and A. H. Piersma (2011). "Comparison of the mouse Embryonic Stem cell Test, the rat Whole Embryo Culture and the Zebrafish Embryotoxicity Test as alternative methods for developmental toxicity testing of six 1,2,4-triazoles." Toxicology and Applied Pharmacology 253(2): 103-111.

des Rieux, A., V. Fievez, M. Garinot, Y. J. Schneider and V. Preat (2006). "Nanoparticles as potential oral delivery systems of proteins and vaccines: a mechanistic approach." J Control Release 116(1): 1-27.

Eisenbrand, G., B. Pool-Zobel, V. Baker, M. Balls, B. J. Blaauboer, A. Boobis, A. Carere, S. Kevekordes, J. C. Lhuguenot, R. Pieters and J. Kleiner (2002). "Methods of in vitro toxicology." Food Chem Toxicol 40(2-3): 193-236. 
Fadeel, B., A. Fornara, M. S. Toprak and K. Bhattacharya (2015). "Keeping it real: The importance of material characterization in nanotoxicology." Biochem Biophys Res Commun 468(3): 498-503.

Flint, O. P. (1993). "In vitro tests for teratogens: desirable endpoints, test batteries and current status of the micromass teratogen test." Reprod Toxicol 7 Suppl 1: 103-111.

Fourches, D., D. Pu, C. Tassa, R. Weissleder, S. Y. Shaw, R. J. Mumper and A. Tropsha (2010). "Quantitative nanostructure-activity relationship modeling." ACS Nano 4(10): 5703-5712.

Frohlich, E. E. and E. Frohlich (2016). "Cytotoxicity of Nanoparticles Contained in Food on Intestinal Cells and the Gut Microbiota." International journal of molecular sciences 17(4).

Genschow, E., G. Scholz, N. Brown, A. Piersma, M. Brady, N. Clemann, H. Huuskonen, F. Paillard, S. Bremer, K. Becker and H. Spielmann (2000). "Development of prediction models for three in vitro embryotoxicity tests in an ECVAM validation study." In vitro \& molecular toxicology 13(1): 51-66.

Genschow, E., H. Spielmann, G. Scholz, I. Pohl, A. Seiler, N. Clemann, S. Bremer and K. Becker (2004). "Validation of the embryonic stem cell test in the international ECVAM validation study on three in vitro embryotoxicity tests." Alternatives to laboratory animals : ATLA 32(3): 209-244.

George, I., S. Vranic, S. Boland, A. Courtois and A. Baeza-Squiban (2015). "Development of an in vitro model of human bronchial epithelial barrier to study nanoparticle translocation.” Toxicol In Vitro 29(1): 51-58.

George, S., T. Xia, R. Rallo, Y. Zhao, Z. Ji, S. Lin, X. Wang, H. Zhang, B. France, D. Schoenfeld, R. Damoiseaux, R. Liu, S. Lin, K. A. Bradley, Y. Cohen and A. E. Nel (2011). "Use of a high-throughput screening approach coupled with in vivo zebrafish embryo screening to develop hazard ranking for engineered nanomaterials." ACS Nano 5(3): 1805-1817.

Ghaisas, S., J. Maher and A. Kanthasamy (2015). "Gut microbiome in health and disease: Linking the microbiome-gut-brain axis and environmental factors in the pathogenesis of systemic and neurodegenerative diseases." Pharmacology \& therapeutics.

Handy, R. D., F. von der Kammer, J. R. Lead, M. Hassellov, R. Owen and M. Crane (2008). "The ecotoxicology and chemistry of manufactured nanoparticles." Ecotoxicology 17(4): 287-314.

Hougaard, K. S., L. Campagnolo, P. Chavatte-Palmer, A. Tarrade, D. Rousseau-Ralliard, S. Valentino, M. V. Park, W. H. de Jong, G. Wolterink, A. H. Piersma, B. L. Ross, G. R. Hutchison, J. S. Hansen, U. Vogel, P. Jackson, R. Slama, A. Pietroiusti and F. R. Cassee (2015). "A perspective on the developmental toxicity of inhaled nanoparticles." Reprod Toxicol 56: 118-140.

Hussain, S. M., D. B. Warheit, S. P. Ng, K. K. Comfort, C. M. Grabinski and L. K. Braydich-Stolle (2015). "At the Crossroads of Nanotoxicology in vitro: Past Achievements and Current Challenges." Toxicol Sci 147(1): 5-16.

Jelinek, R., M. Peterka and Z. Rychter (1985). “Chick embryotoxicity screening test--130 substances tested.” Indian J Exp Biol 23(10): 588-595.

Johnston, H. J., G. Hutchison, F. M. Christensen, S. Peters, S. Hankin and V. Stone (2010). "A review of the in vivo and in vitro toxicity of silver and gold particulates: particle attributes and biological mechanisms responsible for the observed toxicity." Crit Rev Toxicol 40(4): 328-346.

Kish, L., N. Hotte, G. G. Kaplan, R. Vincent, R. Tso, M. Ganzle, K. P. Rioux, A. Thiesen, H. W. Barkema, E. Wine and K. L. Madsen (2013). "Environmental particulate matter induces murine intestinal inflammatory responses and alters the gut microbiome." PLoS One 8(4): e62220.

Kistler, A. (1987). "Limb bud cell cultures for estimating the teratogenic potential of compounds. Validation of the test system with retinoids." Arch Toxicol 60(6): 403-414.

Landsiedel, R., E. Fabian, L. Ma-Hock, W. Wohlleben, K. Wiench, F. Oesch and B. van Ravenzwaay (2012). “Toxico-/ biokinetics of nanomaterials." Arch Toxicol.

Lee, J. A., M. K. Kim, H. J. Paek, Y. R. Kim, M. K. Kim, J. K. Lee, J. Jeong and S. J. Choi (2014). “Tissue distribution and excretion kinetics of orally administered silica nanoparticles in rats." Int J Nanomedicine 9 Suppl 2: 251-260.

Lesniak, A., F. Fenaroli, M. P. Monopoli, C. Aberg, K. A. Dawson and A. Salvati (2012). "Effects of the presence or absence of a protein corona on silica nanoparticle uptake and impact on cells." ACS Nano 6(7): 5845-5857.

Li, H., B. Flick, I. M. Rietjens, J. Louisse, S. Schneider and B. van Ravenzwaay (2015). "Extended evaluation on the ES-D3 cell differentiation assay combined with the BeWo transport model, to predict relative developmental toxicity of triazole compounds." Arch Toxicol. 
Li, H., I. M. Rietjens, J. Louisse, M. Blok, X. Wang, L. Snijders and B. van Ravenzwaay (2015). "Use of the ES-D3 cell differentiation assay, combined with the BeWo transport model, to predict relative in vivo developmental toxicity of antifungal compounds." Toxicol In Vitro 29(2): 320-328.

Li, H., B. van Ravenzwaay, I. M. Rietjens and J. Louisse (2013). "Assessment of an in vitro transport model using BeWo b30 cells to predict placental transfer of compounds." Arch Toxicol 87(9): 1661-1669.

Liu, X., N. Huang, H. Li, Q. Jin and J. Ji (2013). “Surface and Size Effects on Cell Interaction of Gold Nanoparticles with Both Phagocytic and Nonphagocytic Cells.” Langmuir 29(29): 9138-9148.

Mahler, G. J., M. B. Esch, E. Tako, T. L. Southard, S. D. Archer, R. P. Glahn and M. L. Shuler (2012). "Oral exposure to polystyrene nanoparticles affects iron absorption." Nat Nanotechnol 7(4): 264-271.

Meissner, T., A. Potthoff and V. Richter (2009). "Physico-chemical characterization in the light of toxicological effects." Inhal Toxicol 21 Suppl 1: 35-39.

Oberdorster, G., E. Oberdorster and J. Oberdorster (2007). "Concepts of nanoparticle dose metric and response metric." Environ Health Perspect 115(6): A290.

Oberdorster, G., V. Stone and K. Donaldson (2007). “Toxicology of nanoparticles: A historical perspective." Nanotoxicology 1(1): 2-25.

Oksel, C., C. Y. Ma, J. J. Liu, W. T. and W. X. Z. (2015). “(Q)SAR modelling of nanomaterial toxicity: A critical review." Particuology 21: 1-19.

Oksel, C., C. Y. Ma and X. Z. Wang (2015). "Current situation on the availability of nanostructure-biological activity data." SAR and QSAR in environmental research 26(2): 79-94.

Park, E. J., J. Yi, K. H. Chung, D. Y. Ryu, J. Choi and K. Park (2008). "Oxidative stress and apoptosis induced by titanium dioxide nanoparticles in cultured BEAS-2B cells.” Toxicol Lett 180(3): 222-229.

Peters, R., E. Kramer, A. G. Oomen, Z. E. Rivera, G. Oegema, P. C. Tromp, R. Fokkink, A. Rietveld, H. J. Marvin, S. Weigel, A. A. Peijnenburg and H. Bouwmeester (2012). "Presence of nano-sized silica during in vitro digestion of foods containing silica as a food additive." ACS Nano 6(3): 2441-2451.

Piersma, A. H. (2004). "Validation of alternative methods for developmental toxicity testing." Toxicology letters 149(1): 147-153.

Piersma, A. H., G. Janer, G. Wolterink, J. G. Bessems, B. C. Hakkert and W. Slob (2008). "Quantitative extrapolation of in vitro whole embryo culture embryotoxicity data to developmental toxicity in vivo using the benchmark dose approach." Toxicol Sci 101(1): 91-100.

Pietroiusti, A., A. Magrini and L. Campagnolo (2015). "New frontiers in nanotoxicology: Gut microbiota/microbiome-mediated effects of engineered nanomaterials." Toxicology and applied pharmacology.

Poulsen, M. S., T. Mose, L. L. Maroun, L. Mathiesen, L. E. Knudsen and E. Rytting (2015). "Kinetics of silica nanoparticles in the human placenta." Nanotoxicology 9 Suppl 1: 79-86.

Prow, T. W., N. A. Monteiro-Riviere, A. O. Inman, J. E. Grice, X. Chen, X. Zhao, W. H. Sanchez, A. Gierden, M. A. Kendall, A. V. Zvyagin, D. Erdmann, J. E. Riviere and M. S. Roberts (2012). "Quantum dot penetration into viable human skin." Nanotoxicology 6(2): 173-185.

Puzyn, T., D. Leszczynska and J. Leszczynski (2009). “Toward the development of "nano-QSARs": advances and challenges." Small 5(22): 2494-2509.

Reidy, B., A. Haase, A. Luch, K. Dawson and I. Lynch (2013). "Mechanisms of Silver Nanoparticle Release, Transformation and Toxicity: A Critical Review of Current Knowledge and Recommendations for Future." Studies and Applications Materials 6: 2295-2350

Rovida, C. and T. Hartung (2009). "Re-evaluation of animal numbers and costs for in vivo tests to accomplish REACH legislation requirements for chemicals - a report by the transatlantic think tank for toxicology (t(4))." Altex 26(3): 187-208.

Schwiertz, A., D. Taras, K. Schafer, S. Beijer, N. A. Bos, C. Donus and P. D. Hardt (2010). "Microbiota and SCFA in lean and overweight healthy subjects." Obesity 18(1): 190-195.

Sharma, V., D. Anderson and A. Dhawan (2011). "Zinc oxide nanoparticles induce oxidative stress and genotoxicity in human liver cells (HepG2).” J Biomed Nanotechnol 7(1): 98-99.

Sherwood L. (2014). Human Physiology from cells to systems. Canada, Cengage Learning.

Shih, Y. J., C. C. Su, C. W. Chen, C. D. Dong, W. S. Liu and C. P. Huang (2016). "Adsorption characteristics of nano-TiO2 onto zebrafish embryos and its impacts on egg hatching." Chemosphere 154: 109-117.

Shvedova, A. A., A. Pietroiusti, B. Fadeel and V. E. Kagan (2012). "Mechanisms of carbon nanotube-induced toxicity: focus on oxidative stress." Toxicol Appl Pharmacol 261(2): 121-133. 
Spezia, F. and P. C. Barrow (2013). “The teratology testing of cosmetics.” Methods Mol Biol 947: 91-94.

Stokes, W. S. (2015). "Animals and the 3Rs in toxicology research and testing: The way forward." Human \& experimental toxicology 34(12): 1297-1303.

Taylor, A. A., I. M. Marcus, R. L. Guysi and S. L. Walker (2015). "Metal oxide nanoparticles induce minimal phenotypic changes in a model colon gut microbiota." Environ Eng Sci 32: 602-612.

Tenzer, S., D. Docter, J. Kuharev, A. Musyanovych, V. Fetz, R. Hecht, F. Schlenk, D. Fischer, K. Kiouptsi, C. Reinhardt, K. Landfester, H. Schild, M. Maskos, S. K. Knauer and R. H. Stauber (2013). "Rapid formation of plasma protein corona critically affects nanoparticle pathophysiology." Nat Nanotechnol 8(10): 772-781.

Toropova, A. P., A. A. Toropov, A. M. Veselinovic, J. B. Veselinovic, E. Benfenati, D. Leszczynska and J. Leszczynski (2016). "Nano-QSAR: Model of mutagenicity of fullerene as a mathematical function of different conditions." Ecotoxicol Environ Saf 124: 32-36.

Tsai, F. and W. J. Coyle (2009). “The microbiome and obesity: is obesity linked to our gut flora?" Current gastroenterology reports 11(4): 307-313.

van Aerle, R., A. Lange, A. Moorhouse, K. Paszkiewicz, K. Ball, B. D. Johnston, E. de-Bastos, T. Booth, C. R. Tyler and E. M. Santos (2013). "Molecular mechanisms of toxicity of silver nanoparticles in zebrafish embryos." Environ Sci Technol 47(14): 8005-8014.

Van der Jagt, K., S. Munn, J. Torslov and J. de Bruijn (2004). "Alternative Approaches can reduce the use of test animals under REACH. Addendum to the report "assessment of additional testing needs under REACH. Effects of (Q)SARs, Risk Based Testing and Volunary Industry Initiatives.” JRC Report EUR: 25.

van der Zande, M., R. J. Vandebriel, M. J. Groot, E. Kramer, Z. E. Herrera Rivera, K. Rasmussen, J. S. Ossenkoppele, P. Tromp, E. R. Gremmer, R. J. Peters, P. J. Hendriksen, H. J. Marvin, R. L. Hoogenboom, A. A. Peijnenburg and H. Bouwmeester (2014). "Sub-chronic toxicity study in rats orally exposed to nanostructured silica." Part Fibre Toxicol 11: 8.

van Kesteren, P. C., F. Cubadda, H. Bouwmeester, J. C. van Eijkeren, S. Dekkers, W. H. de Jong and A. G. Oomen (2014). "Novel insights into the risk assessment of the nanomaterial synthetic amorphous silica, additive E551, in food." Nanotoxicology: 1-10.

Walczak, A. P., E. Kramer, P. J. Hendriksen, P. Tromp, J. P. Helsper, M. van der Zande, I. M. Rietjens and H. Bouwmeester (2014). "Translocation of differently sized and charged polystyrene nanoparticles in in vitro intestinal cell models of increasing complexity." Nanotoxicology: 1-9.

Westmeier, D., R. H. Stauber and D. Docter (2016). "The concept of bio-corona in modulating the toxicity of engineered nanomaterials (ENM).” Toxicol Appl Pharmacol 299: 53-57.

Yoo, M. H., Y. C. Rah, J. Choi, S. Park, H. C. Park, K. H. Oh, S. H. Lee and S. Y. Kwon (2016). "Embryotoxicity and hair cell toxicity of silver nanoparticles in zebrafish embryos." International journal of pediatric otorhinolaryngology 83: 168-174.

zur Nieden, N. I., G. Kempka and H. J. Ahr (2004). "Molecular multiple endpoint embryonic stem cell test--a possible approach to test for the teratogenic potential of compounds." Toxicol Appl Pharmacol 194(3): 257-269. 


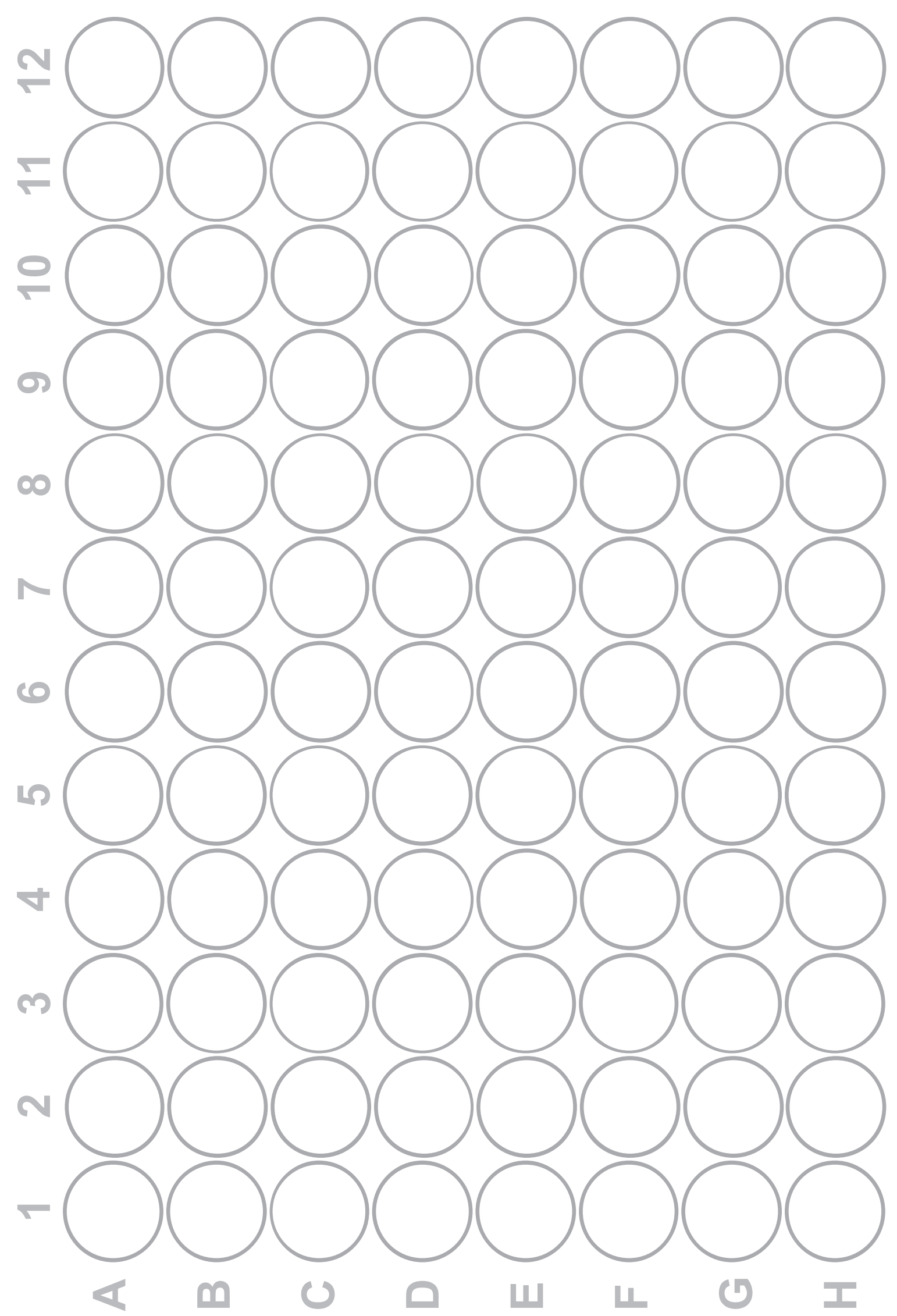




\section{Chapter 8}

Summary 


\section{SUMMARY}

The production of nanoparticles (NPs) has increased in the last decades and the number of products in which NPs are being incorporated is still growing. The rapid increase of nanotechnology has several benefits for society, yet there is an increasing concern that exposure to NPs may result in significant adverse health effects. Since NPs are incorporated in a variety of consumer products, it is likely that the general population will be exposed to NPs, via NP-containing products such as personal care products, food and food packaging materials, textiles and medicines. It would be desirable that the safety and risk assessment of NPs could be largely based on studies using in vitro models instead of in vivo models as this would reduce the use of test animals, costs and time required to test the large numbers of NPs. The aim of the present thesis was to investigate the potential of in vitro testing strategies to detect hazards of NPs, focusing on toxicokinetic as well as toxicodynamic endpoints. Toxicokinetic studies focused on translocation of NPs in in vitro models of the placental barrier, while toxicodynamic studies were directed at two endpoints that represent potential hazards of NPs that have not yet been well characterized including: developmental toxicity and immunotoxicity.

Chapter 1 introduced the aim of the thesis and the various in vitro models and NPs used in the studies performed. In the present thesis different types of NPs were used. Polystyrene nanoparticles (PS-NPs) were selected because of their commercial availability, with high quality and a wide variety of available physicochemical properties like surface charge, and fluorescent labeling enabling easy detection in toxicokinetic (translocation) studies. Several metal (oxide) NPs were selected as well, of which some are possible constituents of food additives like $\mathrm{TiO}_{2}, \mathrm{Fe}_{2} \mathrm{O}_{3}, \mathrm{SiO}_{2}$ and $\mathrm{Ag}$. Other metal oxide NPs that were selected were $\mathrm{Mn}_{2} \mathrm{O}_{3}$, $\mathrm{CuO}, \mathrm{Cr}_{2} \mathrm{O}_{3}, \mathrm{CoO}$ and $\mathrm{NiO}$ to which we may be exposed via products like paints, catalysts, construction materials, coatings and batteries.

Chapter 2 of the present thesis provided a review about the performance of in vitro models for studies on toxicokinetics of NPs. The chapter described models that mimic the different barriers of the human body, with a focus on the lung, gut, skin and placental barrier, thus providing an overview of the state-of-the-art of using in vitro model systems, ranging from single cell-type monolayer to multi-cell (3D) models, to characterize the translocation of NPs across these different barriers. In this chapter it was concluded that at the current state-ofthe-art, the in vitro models do not yet allow prediction of absolute transfer rates but they do support the definition of relative transfer rates and can thus help to reduce animal testing by setting priorities for subsequent in vivo testing. The use of in vitro models is very promising since they are currently further improved to mimic the in vivo situation more closely by, for example, using co-cultures of different cell types and implementing them in a microfluidic chip format. When these models are further validated by testing exactly the same NPs in an in 
vivo set-up and in the in vitro model, they could be even used to determine the internal exposure (bioavailability) of NPs in an absolute way, besides to set priorities for NP toxicity testing.

Chapter 3 showed the results of placental translocation of NPs as an important toxicokinetic aspect, since part of the toxicodynamic studies of the present thesis were directed at developmental toxicity testing of NPs. In order to obtain insight in toxicity and translocation of NPs across the placental barrier, cytotoxicity and translocation was studied for one positively and two negatively charged PS-NPs of $50 \mathrm{~nm}$ in an in vitro model of the placenta. The model consisted of BeWo b30 cells, derived from a human choriocarcinoma grown on a transwell insert forming a cell layer that separates an apical from a basolateral compartment. PS-NPs were characterized with respect to size, surface charge, morphology and protein corona. In this study it appeared that in spite of similar size, surface charge and type of proteins in the protein corona, the differently charged NPs displayed a remarkable difference in cytotoxicity, with only the PS-NPs with an original positive charge inducing cytotoxicity. Translocation of PS-NPs appeared not to be related to PS-NP charge alone. A remarkable difference in translocation was found between the two $50 \mathrm{~nm}$ negatively charged PS-NPs that were obtained from different manufacturers. For the negatively charged PS-NPs from Polysciences, the apparent permeability coefficient (Papp) was slightly higher than that of amoxicillin, a compound that is known to be translocated over the in vitro BeWo b30 placental barrier and the ex vivo human placental barrier to only a limited extent. When testing the negatively charged PS-NPs from Magsphere, no PS-NPs could be detected in the basolateral compartment upon adding a concentration of $10 \mu \mathrm{g} / \mathrm{ml}$ to the apical compartment and 24 hours incubation. Since none of the characterized parameters, including size, surface charge and protein corona revealed remarkable differences between the two negatively charged NPs, the difference may originate from the chemical groups on the surface of the NPs generating the negative charge. The general conclusion from this study was that the in vitro BeWo b30 model can be used as a fast method to get an initial qualitative impression about the capacity of NPs to translocate across the placental barrier and to set priorities for further in vivo studies on translocation of NPs to the fetus.

Chapter 4 investigated whether the same PS-NPs as tested in chapter 3 for placental translocation, are able to cause in vitro developmental toxicity in the ES-D3 cell differentiation assay of the embryonic stem cell test (EST) focusing also on the effect that charge may have. The study showed that the two negatively charged PS-NPs did not show any effect in the ES-D3 cell differentiation assay up to the highest concentration tested while the positively charged PS-NP showed a concentration-dependent inhibition of ES-D3 cell differentiation. However, effect concentrations in the ES-D3 cell differentiation assay were close to cytotoxic concentrations, which indicated that the inhibition of the ES-D3 cell differentiation may be due to cytotoxic effects of the positively charged PS-NPs. This indicated that the inhibition of the ESD3 cell differentiation by the positively charged PS-NPs may be caused by non-specific effects. 
A literature search was done on the reported potencies of other NPs in the ES-D3 cell differentiation assay, in order to assess whether effects of NPs in the ES-D3 cell differentiation assay may also be related to physicochemical properties other than surface charge, such as size or coating. Although the experiments in chapter 3 of the present thesis showed that positively charged PS-NPs are more toxic than negatively charged PS-NPs, it appeared that this may not be generalizable to other NPs. This follows from the fact that $\mathrm{SiO}_{2}, \mathrm{Ag}$ and $\mathrm{TiO}_{2} \mathrm{NPs}$ that were reported to inhibit ES-D3 cell differentiation were negatively charged, while the negatively charged PS-NPs of the present study did not affect ES-D3 cell differentiation. To obtain better insight in the role of NP surface charge on developmental toxicity more studies are required that assess the effects of NPs with different charge that are made of the same core material. Although the limited data available indicate that charge, size and coating of NPs may be important characteristics that determine the developmental toxicity potential of NPs, more (systematic) studies are needed to assess how physicochemical characteristics of NPs relate to their developmental toxicity. This information may help to prioritize NPs for in vitro and in vivo developmental toxicity testing.

In chapter 5, toxic effects of a series of metal (oxide) NPs were tested in macrophage RAW264.7 cells in order to obtain insight in effect of these NPs on cells of the innate immune response. In these macrophage RAW264.7 cells the effects of the metal (oxide) NPs were characterized on cell viability, TNF- $\alpha$ production and mitochondria-related parameters like production of reactive oxygen species (ROS), mitochondrial permeability transition pore (MPTP) opening, and intracellular ATP levels. The results showed that $\mathrm{SiO}_{2}$ and $\mathrm{Ag}$ NPs were the most toxic ones among the tested food-related NPs and that $\mathrm{TiO}_{2}, \mathrm{Fe}_{2} \mathrm{O}_{3}, \mathrm{Cr}_{2} \mathrm{O}_{3}, \mathrm{CoO}$ and $\mathrm{NiO} \mathrm{NPs}$ showed no effects on the selected endpoints up to the highest concentration tested $(200 \mu \mathrm{g} /$ $\mathrm{ml}$ ). Altogether, results obtained in chapter 5 showed no or limited effects of the NP formulations of metal (oxide) food additives on cell viability, ROS production, MPTP opening, ATP levels and TNF- $\alpha$ production in RAW264.7 macrophages. Effects were only observed at high concentrations that may not be physiologically relevant, indicating that related adverse effects upon exposure to the respective NPs in vivo may be limited.

Of the NPs tested in chapter 5, Ag NPs were among the NPs showing most effects. The volume of Ag NPs-based products entering the market is relatively high, making the risk assessment of Ag NPs a priority. Therefore to elucidate the factors that drive Ag NPs' potential to pose health risks, chapter 6 assessed the effects of specifically synthetized Ag NPs with two different sizes (20 and $50 \mathrm{~nm}$ ) and with three different surface coatings (chitosan: Ag NP_Chit, bovine serum albumin: Ag NP_BSA, polyvinylpyrrolidone: Ag NP_PVP) in the same in vitro RAW264.7 model. The effect of surface coating and size of NPs was characterized on cell viability, TNF- $\alpha$ production, ROS production, MPTP opening, and intracellular ATP levels. Decreased cell viability was observed for all Ag NPs tested. The results from MTT, ATP and 
MPTP opening assays showed that adverse effects from exposure to the tested Ag NPs on these endpoints were similar irrespective of surface coating or size. However, for ROS production and TNF- $\alpha$ induction, significant differences were found with variations in the physicochemical properties of the tested Ag NP. The role of surface coating/charge in influencing TNF- $\alpha$ induction was demonstrated by the fact that only negatively charged Ag NP_BSA induced TNF- $\alpha$ production (80x higher than control). Further, significant ROS induction was only observed with the $20 \mathrm{~nm}$ positively charged Ag NP_Chit. Overall, the negatively charged Ag NP_BSA appeared to be most potent in inducing adverse effects on the macrophages. This toxicity of the negatively charged Ag NPs is the opposite of what was found in chapter 3 for the PS-NPs, since the positively charged PS-NPs appeared to be the most cytotoxic to BeWo cells. So this points again to the direction that the effect of surface charge may vary with the type of NPs investigated, indicating that more studies with other NPs with different core material and with different charges are required.

Finally chapter 7 of the thesis presented a general discussion and some future perspectives for use of in vitro models in defining hazards of NPs. It was concluded that taken together, the present thesis provided further evidence of the influence of physicochemical properties of NPs in driving toxicity in in vitro models. However, the determination of the fate and toxicity of NPs using in vitro or in vivo models is a challenge that needs further evaluation. A combination of several factors likely play a role in determining the outcome of exposure including factors like NP core material and presence and type of coating agents resulting in various physicochemical properties (size, charge, etc.). This appears to hamper conclusive evaluation of the role of physicochemical characteristics of NPs in their potential hazards and risks so far. The results obtained do show however that in vitro assays can detect differences in potential hazards posed by NPs. Therefore it is concluded that the results of the work presented in this thesis will contribute to the further development and use of non-animal based testing strategies for safety testing of NPs providing insight into selected potential hazards of the tested NPs. 


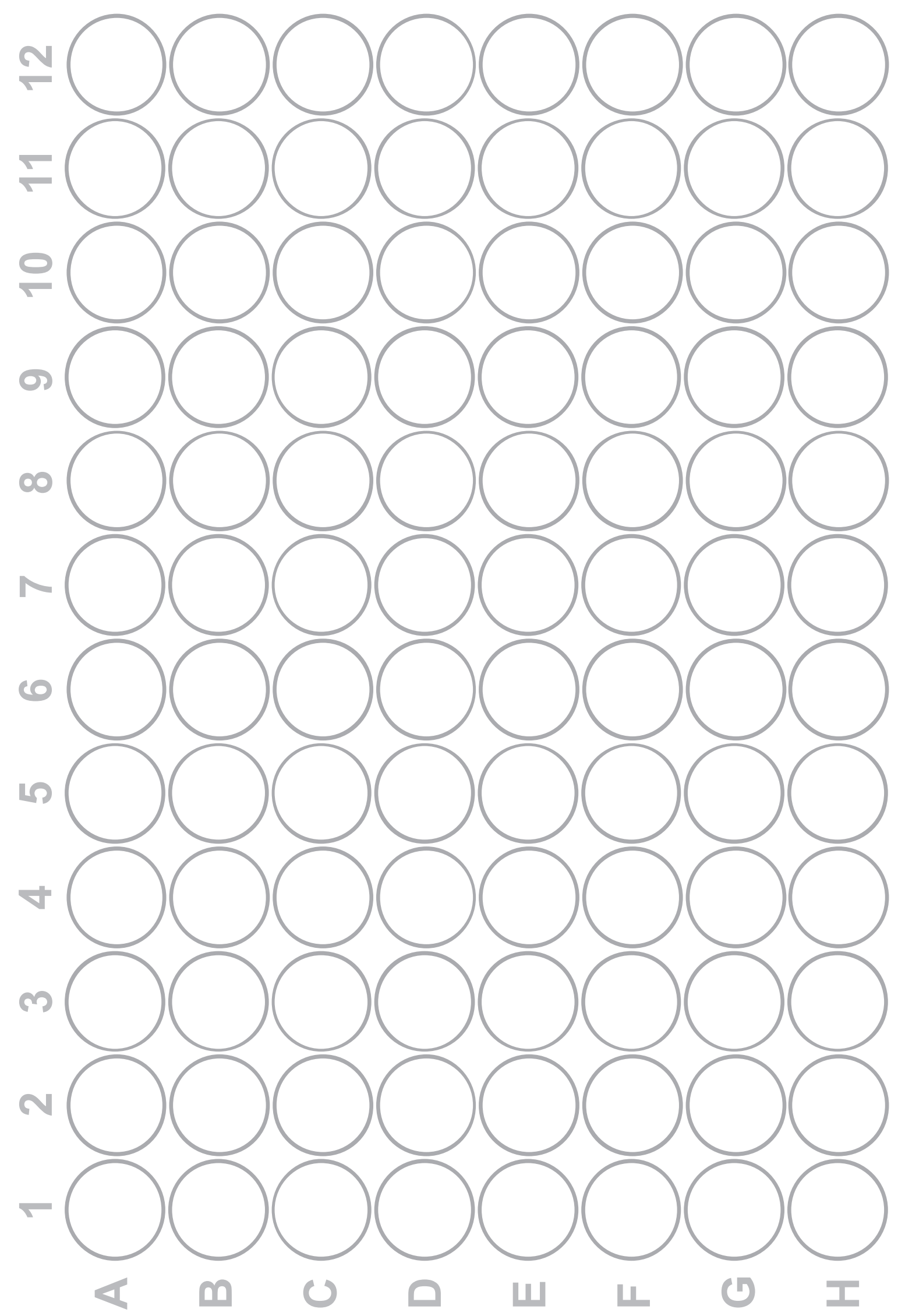




\section{Appendix}

Acknowledgements

About the author

List of publications

Overview of completed training activities 


\section{ACKNOWLEDGEMENTS}

Het is zover, na ruim 4 jaar onderzoek doen en schrijven, mijn boekje is af! Er zijn veel mensen die een rol hebben gespeeld bij de totstandkoming van mijn proefschrift. Ik ben daarvoor veel dank verschuldigd aan mijn begeleiders, collega's, familie en vrienden. In het bijzonder wil ik allereerst mijn supervisors bedanken.

Ivonne, bedankt dat je me de mogelijkheid gaf om dit project te doen en bedankt voor alle begeleiding en support afgelopen jaren. Als promotor heb je me enorm geholpen om de grote lijnen van het proefschrift vast te houden. Ik heb ontzettend veel geleerd van jouw kritische en kundige blik op mijn resultaten. De snelle feedback en constructieve commentaren op abstracts, manuscripten, presentaties en posters die je altijd gaf waren als erg leerzaam en inspirerend ervaren, bedankt! Jochem, als directe begeleider en co-promotor maakte je altijd direct tijd vrij om mijn vragen te beantwoorden en mijn manuscripten te bekritiseren en advies te geven. Bedankt voor de discussies die we hadden en waardoor ik leerde ook vanuit een andere invalshoek naar de dingen te gaan kijken. Ik vond het fijn dat ik met je samen mocht werken de afgelopen jaren. Ik wil co-promotor Nico bedanken voor de fijne samenwerking en je waardevolle bijdrages in de latere stadia van het project. Bedankt voor je kritische blik op de manuscripten en fijne feedback daarop.

Daarnaast wil ik ook NanonextNL bedanken als sponsor van het project. Tevens alle Nano nextNL collegas van andere instituten die betrokken waren bij het project bedankt voor de fijne samenwerking.

Furthermore, I would like to thank all my colleagues at Toxicology. My office mates Henrique, Myrthe, Jonathan and Hequn, thank you for the nice company during the years, lively discussions about many topics like food and 'dutch habbits' and fun in our rooms. My nano-buddies Sunday, Merel, Sourav, thank you for the nice collaborations and nano talks and other discussions and fun during conferences we have visited sometimes. Agata, thank you for all the nano-wisdom we shared! I enjoyed all the coffees and lunches we had, both in Wageningen and now in Utrecht which I am sure we will continue. Alexandros, thank you for the fun talks about music. The CD compilations with some heavy metal music are still in my car and they have kept me awake for sure, during long traveling hours. Lenny and Ignacio, I am very happy that you are my paranymphs. Thank you for being great colleagues and friends! Thank you Irene, Gré and Lidy for helping out with ordering a variety of chemicals and labware and for your help with all kinds of administrative things. My buddy Hans, thank you so much for not only your help in the lab during experimental rush hours, but also for the nice company and talks! Thanks Laura, Bert, Sebas for your technical assistance and other help. Also many thanks to all other (and former) TOX-colleagues Abdul, Agata, Ashraf, Amer, Ans, Arif, Ar- 
tem, Aziza, Barae, Erryana, Gerrit, Ignacio, Jac, Jia, Justine, Karsten, Letty, Lu, Marcia, Marije, Marta, Myrthe, Myrtho, Mengying, Mebrahtom, Nico, Nynke, Reiko, Rozaini, Rungnapa, Ruud, Sophie, Suzanne, Tinka, Wasma et al. It has been a great pleasure working with you. Several students were involved in my project. Vasileios, Leonie, Bernice, Edith, Gang, George, Heike, Aikaterini, Sarah, and Ignacio and Ashraf of which the last two of you are currently doing their own PhD project. Thank you for the nice collaboration and all the work you did. Miljards of cells, miljons of nanoparticles, in the end we succeeded to know some more about the effects of nanoparticles. I enjoyed supervising you all and wish you the best in your future career. I would also like to express my gratitude to all co-authors I have not already mentioned. Thank you for your hard work that brought us successful output.

I would also like to express my gratitude to the committee members Prof. Dr A.A. Koelmans, Prof. Dr A.H. Piersma, Dr A.G. Oomen and Dr T. Hamers, for the time and efforts they took to evaluate this thesis.

Marije, Christa en Noortje, jullie waren daar altijd voor een luisterend oor en hielpen me herinneren dat er meer in het leven is dan 'nano'. Bedankt voor al die momenten waar we zoveel plezier hadden, de oldschool high-beers in Nijmegen, de vele etentjes en 'apekop' shotjes erna, de leuke uitjes (o.a. York, Londen en het jaarlijkse Zwarte Cross) tijdens de soms stressvolle periodes van mijn $\mathrm{PhD}$. Het was top en ik hoop dat we deze tradities voort gaan zetten!

Vrienden en familie, ouders en broertje, die al mijn verhalen over nanodeeltjes en experimenten moesten aanhoren, bedankt voor jullie interesse en ondersteuning. Pap en mam, fijn om te weten dat jullie trots op me zijn. Bedankt voor jullie steun afgelopen jaren en voor alle keren dat er weer een heerlijke (indonesische) maaltijd klaarstond of een logeerplek wanneer ik eens tot laat moest werken.

Als allerlaatste wil ik mijn man Renzo bedanken, de afgelopen jaren was jij mijn praatpaal, mijn rots in de branding. Je hebt altijd veel begrip gehad en bedankt voor je aandacht, geduld, grappen, gezelligheid en vele avondjes 'rocken' danwel op een metalconcert/festival ofwel op een rockband-party thuis, die voor ontspanning zorgden. Met name de laatste fase van mijn $\mathrm{PhD}$ was roerig en was erg stressvol met allerlei zaken die tegelijk kwamen: de verhuizing, de verbouwingen, de voorbereidingen van de bruiloft, het afronden van het proefschrift etc. Maar we hebben ons er uiteindelijk doorheen geslagen en ik kijk uit naar onze verdere toekomst samen! 


\section{ABOUT THE AUTHOR}

Samantha Kristina Kloet was born on November $9^{\text {th }}, 1987$ in Arnhem and grew up in Elst. After her graduation from secondary school at the Olympus College (Arnhem, The Netherlands) in 2005, she started to study Biomedical Sciences at the Radboud University (Nijmegen, The Netherlands). During her bachelor she did an internship at the department of membrane biochemistry at the Nijmegen Centre for Molecular Life Sciences (NCMLS, Nijmegen, The Netherlands), for wich she won the 'Bex price' for outstanding accomplishment of the bachelor internship. After obtaining her Bachelor's degree in 2008 she started the MSc programs Pathobiology and Toxicology at the Radboud Univeristy that same year. During her MSc, she performed two internships; one at the Division of Toxicology (Wageningen University and Research Center) and another one at the department of Molecular Ophthalmogenetics (Amsterdam, Netherlands Institute for Neuroscience). After graduation from her MSc degree, she started her $\mathrm{PhD}$ research described in this thesis at the Division of Toxicology at Wageningen University. During her $\mathrm{PhD}$, Samantha followed a postgraduate education program and registered as toxicologist-in-training, to obtain a registration as European Registered Toxicologist (ERT) after graduation. During the period of her PhD she was a member of the board committee at the Division of Toxicology and she was an editorial board member of the newsmagazine (TCDD) of the Dutch Society of Toxicology (NVT). In 2016, Samantha is working as a toxicological risk assessor at the National Institute for Public Health and the Environment (RIVM, Bilthoven). 


\section{LIST OF PUBLICATIONS}

Kloet SK, Walczak AP, Louisse J, van den Berg HHJ, Bouwmeester H, Tromp P, Fokkink RG, Rietjens IMCM . (2015). "Translocation of positively and negatively charged polystyrene nanoparticles in an in vitro placental model.” Toxicology In Vitro.

Kloet SK, van den Berg HHJ, Fransen LFH, Estruch IM, Vasileiadis V, de Haan L, Rietjens IMCM, Louisse J. (2016). "Assessment of the potential developmental toxicity of positively and negatively charged polystyrene nanoparticles using the embryonic stem cell differentiation assay." Submitted to Toxicology Letters.

Kloet SK, Makama S, van den Berg HHJ, Wu G, Saviolakis G, Vogt H, Manolesou A, van den Brink NW, Rietjens IMCM, Louisse J. (2016). "Effects of nanoparticle forms of metal (oxide) food additives and related nanoparticles on cell viability, TNF- a production and mitochondria-related parameters in RAW264.7 macrophages". Submitted to Toxicology In Vitro.

Makama S, Kloet SK, Piella J, van den Berg HHJ, de Ruijter NCA, Puntes VF, Rietjens IMCM, van den Brink NW. (2016) "Cellular interactions of silver nanoparticles with systematic variation in size and surface coating with macrophage RAW 264.7 cells". Submitted to Nanotoxicology.

Braakhuis HM, Kloet SK, Kezic S, Kuper F, Park MVDZ, Bellmann S, van der Zande M, Le Gac S, Krystek P, Peters RJB, Rietjens IMCM, Bouwmeester H. (2015). "Progress and future of in vitro models to study translocation of nanoparticles." Archives of Toxicology.

Gijsbers L., Man HY, Kloet SK, de Haan LH, Keijer J, Rietjens IMCM, van den Burg B, Aarts JM. (2011). "Stable reporter cell lines for peroxisome proliferator-activated receptor $\gamma$-mediated modulation of gene expression." Analytical Biochemistry.

Gijsbers L, van Eekelen HD, de Haan LH, Swier JM, Heijink NL, Kloet SK, Man HY, Bovy AG, Keijer J, Aarts JM, van der Burg B, Rietjens IMCM. (2013). "Induction of peroxisome proliferator-activated receptor $\gamma(\operatorname{PPAR} \gamma)$-mediated gene expression by tomato (Solanum lycopersicum L.) extracts." Journal of Agricultural and Food Chemistry. 


\section{OVERVIEW OF COMPLETED TRAINING ACTIVITIES}

\section{Discipline specific courses}

Risk Assessment, Postgraduate Education in Toxicology (PET) (2012)

Epidemiology, PET (2012)

Medical and Forensic Toxicology, PET (2012)

Food Toxicology, PET (2013)

Environmental toxicology, Wageningen University (WUR) (2013)

Risk Analysis and Technology Assessment, NanoNextNL (2013)

\section{Meetings and Conferences}

MicroNanoConference, Ede, The Netherlands (2011)

First Joint German-Dutch Meeting of SOT, Düsseldorf, Germany (2012) (poster)

NanoNextNL RATA meeting, Bilthoven, The Netherlands (2012) (poster)

NanoNextNL meeting, Wageningen, The Netherlands (2013) (oral)

$7^{\text {th }}$ International Nanotoxicology Congress, Antalya, Turkey (2014) (poster)

Nanocity conference, De Fabrique, Utrecht, The Netherlands (2014) (poster)

Nanocity conference, De Rijtuigenloods, Amersfoort, The Netherlands (2015) (poster)

\section{General courses}

VLAG PhD week (2012)

How to give and receive feedback, Young AFSG (2012)

Project and time management, WGS (2012)

Competence assessment, WGS (2012)

Voice matters - V\&PT, WGS (2013)

Philosophy and Ethics of Food Science and Technology, WGS (2014)

Mobilising your - scientific - network, VLAG (2014)

Intellectual Property \& Valorisation Awareness, NanoNextNL (2014)

\section{Optional activities}

Preparing PhD research proposal (2012)

Attending scientific presentations (Division of Toxicology)

PhD excursion UK (2013)

Approved by the Graduate School VLAG. 
This thesis is part of NanoNextNL, a micro and nanotechnology innovation consortium of the Government of the Netherlands and 130 partners from academia and industry. More information on www.nanonextnl.nl.

Financial support from Wageningen University for printing this thesis is gratefully acknowledged.

Cover and Layout design: Jardine Media | jardinemedia.com

Printing: Ridderprint, Ridderkerk (NL) | ridderprint.nl

Samantha K. Kloet, 2016 